Portland State University

PDXScholar

Dissertations and Theses

Dissertations and Theses

Summer 8-16-2017

\title{
Development of Physics Curriculum for Pre-Health Students
}

\author{
Elliot Eckman Mylott \\ Portland State University
}

Follow this and additional works at: https://pdxscholar.library.pdx.edu/open_access_etds

Part of the Curriculum and Instruction Commons, Medical Education Commons, and the Physics Commons

Let us know how access to this document benefits you.

\section{Recommended Citation}

Mylott, Elliot Eckman, "Development of Physics Curriculum for Pre-Health Students" (2017). Dissertations and Theses. Paper 3779.

https://doi.org/10.15760/etd.5663

This Dissertation is brought to you for free and open access. It has been accepted for inclusion in Dissertations and Theses by an authorized administrator of PDXScholar. Please contact us if we can make this document more accessible: pdxscholar@pdx.edu. 
Development of Physics Curriculum for Pre-Health Students

by

Elliot Eckman Mylott

A dissertation submitted in partial fulfillment of the requirements for the degree of

\author{
Doctor of Philosophy \\ in \\ Applied Physics
}

Dissertation Committee:

Ralf Widenhorn, Chair

Erik Bodegom

Jack Barbera

Dean Atkinson

Karen Marrongelle

Justin Dunlap

Portland State University

2017 


\begin{abstract}
Many pre-health students are required to take introductory physics as undergraduates, though they often struggle to see the relationship between medicine and what they learn in these courses. In order to help students make that connection, reformed curriculum was adopted that teaches physics though the context of biomedicine. This dissertation will discuss the development, implementation, and assessment of the reformed curriculum for the introductory and intermediate level physics courses that targets the needs of pre-health students.
\end{abstract}

The curriculum created during this project include laboratory activities, multimedia content, and other instructional materials all of which present physics in biomedical contexts. The laboratory activities focus on exploring the physical principles behind common medical devices or concepts such as body composition analyzers or computed tomography. This often required researching, designing, and building devices for use in the classroom. Videos interviews with biomedical experts detail how physics is used in their fields. The texts written for these courses serve as a fundamental scientific introduction to the physical concepts and technical discussions of their application in biomedicine. An online homework platform allows for the implementation of a flipped classroom. Homework integrates the material, probing both conceptual understanding and problem solving. Multiple forms of assessment have been used to improve the content and clarity of the curriculum.

The research for this project includes a study of the impact of these course reforms on students' attitudes toward physics. Shifts in attitudes were assessed using the Colorado Learning Attitudes about Science Survey (CLASS), course surveys, student interviews, and conceptual quizzes. Data was collected from students in the reformed 
course and a concurrent course taught using a traditional physics curriculum that does not have a focus on biomedicine.

The results show that students' attitudes were affected by the reforms in multiple ways including students' ability to contextualize physical phenomenon through biomedical applications. Direct responses from the students indicated that they appreciated that the course included biomedically relevant content. They stated that the course had helped them to make connections with physics that they were not able to make in previous physics classes. However, a portion of the students qualified their approval of the course reforms by stating, for example, that they felt they were missing out on other topics due to the biomedical focus.

There is evidence that other factors such as class meeting time could have played a role in students' attitudes as well. Students were surveyed multiple times throughout the year. Results of these surveys show that students' attitudes tend decrease during fall term, but improve after winter and spring term. These results suggest that greater gains in favorable attitudes could be achieved by course reforms in fall term, where positive attitudes are at a minimum.

Illustration-based quizzes were administered to assess students' conceptual understanding and contextualization of different physical phenomena. The quizzes featured open-ended prompts about illustrations similar to those often seen in physics instruction. Few significant differences in conceptual understanding were found between students in the reformed and traditional courses. However, students in the reformed course were more likely to cite biomedical applications of the physical phenomena.

These course reforms teach physical principles through their application to biomedical technology and have positively impacted students' appreciation for the relationship between physics and biomedicine. 


\section{Acknowledgements}

I would like to thank my dissertation committee for their thoughtful comments during the project Ralf Widenhorn, Erik Bodegom, Dean Atkinson, Jack Barbera, Justin Dunlap, and Karen Marrongelle. They have provided extremely meaningful advice about education research and the project is all the stronger because of it.

I want to give a very special thank you to my adviser Ralf Widenhorn. It is difficult to think of a single thing I have done at PSU that Ralf hasn't been a part of. I started working with Ralf nearly a decade ago as an undergraduate learning assistant. He gave me a recommendation letter for my study abroad program. When I returned I started working on the early stages of his course reforms for pre-health students. I joined his lab as a graduate student and worked on digital imagers for my masters, while collaborating on physics education research on the side. He had the confidence to me in charge of logistics for the workshops of the American Association of Physics Teachers conference in Portland. The list goes on and on. I am extremely fortunate to have had Ralf as an adviser and have greatly benefited from his guidance and encouragement.

I would like to thank Warren Christensen from North Dakota State University for sharing his expertise in physics education research and for his work during the study including his help with the interviews and reviews of the analysis. The student researchers at NDSU also deserve credit for their hard work in the analysis and inter-

pretation of the results of the study. Thank you to Daron Dykes, Jordan Brainard, and Matt Urich.

A very special thank you to the instructors of the reformed introductory courses Justin Dunlap and Priya Jamkhedkar for their support of the study. This project 
continues to benefit from their contributions as teachers, content creators, and researchers. Course reforms can not be successful without engaged, caring educators like them.

Many students from PSU and elsewhere assisted with the project over the years, which has greatly benefited from their contributions. Thank you to Tanya Korzun and Maryana Lazaruko for their thoughtful analysis of the survey data. I want to thank Gavin Graeper and Chelsea Page from OHSU for their work in developing and improving the course materials. Thank you to Sara Wiley, Grace Van Ness, Sam Matz, Ben Galvin, Eric Eddy, Aaron Heston, and Kathryn Stinson for their work in creating multimedia content. Thank you to Ellynne Kutschera, Ryan Klepetka, Otto Zietz, Casey Norlin, Misti Byrd, Sabrina Hoffman, and Lester Lampert for thier work in developing the laboratory activities. And thank you to Jessa Lulich, Jeff Halcott, James Salamanca, James Johnson, and Elizabeth Anderson for their work in researching and evaluating the course reforms.

The support staff at PSU are a vital part of the physics department. No project can be successful without their work. I want to thank Leroy Laush, Marc Nisenfeld, and Alex Chally from the science shop for their hard work and technical expertise in the creation of the materials we use in our labs. Thank you to Kim Doty-Harris, Laurie Tull, and Alex Christie from the physics office for their greatly appreciated assistance in navigating the policies and procedures of the PSU financial system.

I want to thank my partner Amanda Mather for her love, support, and encouragement during my research. Thank you to my family Grace Weatherford, Liz Moorehead, and Jim Mylott for their encouragement during my entire career at PSU.

This work was supported by grants (DUE-1141078 and DUE-1431447) from the National Science Foundation. 


\section{Table of Contents}

Abstract

Acknowledgements $\quad$ iii

List of Tables $\quad$ xi

List of Figures $\quad$ xiii

1 Introduction and Background Information 1

1.1 Introductory Physics for Pre-health and Life Science Students . . . . 1

1.2 Physics for Pre-Health Students at PSU . . . . . . . . . . . . 3

1.3 The Role of Interest in Learning . . . . . . . . . . . . . . . . 4

1.4 The Role of Interest in Introductory Physics for the Life Sciences . . 5

1.5 Research Questions ................... 6

1.6 Structural Overview . . . . . . . . . . . . . . . . . 8

2 Descriptions of Course Reforms $\quad 10$

2.1 Laboratories . . . . . . . . . . . . . . . . . . . . . 11

2.1.1 Bioelectrical Impedance Analysis . . . . . . . . . . . . . . . . 13

2.1.2 Computed Tomography . . . . . . . . . . . . . . . . . 21

2.1.3 Optics of the Human Eye . . . . . . . . . . . . . 27

2.1.4 Radiography . . . . . . . . . . . . . . . . 32

2.1.5 Pulse Oximetry . . . . . . . . . . . . . . . 37

2.1.6 Electrocardiogram . . . . . . . . . . . . . . 39 
2.1.7 Kinesthetic Activities . . . . . . . . . . . . . . . . . 42

2.2 Multimedia Content . . . . . . . . . . . . . . . . . . 48

$2.2 .1 \quad$ Expert Interviews . . . . . . . . . . . . . . . . . . . . . . 48

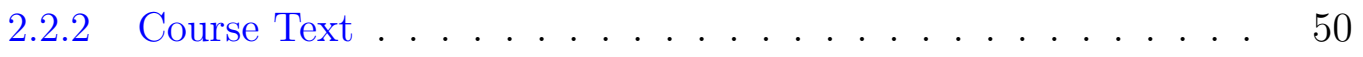

2.2 .3 FlipItPhysics . . . . . . . . . . . . . . . . . . . . 51

2.2.4 Multimedia Modules Analysis . . . . . . . . . . . . 55

2.3 Reformed Introductory Physics Course . . . . . . . . . . . . 57

2.3 .1 Course Structure . . . . . . . . . . . . . . . . . . . 57

2.3.2 Use of Multimedia Modules in the Curriculum . . . . . . . 59

2.3 .3 Laboratory Activities . . . . . . . . . . . . . . . . . . 59

2.3 .4 Student Surveys . . . . . . . . . . . . . . . . . . . . . . 61

2.3.5 CLASS Analysis . . . . . . . . . . . . . . . 64

2.4 Intermediate Level Physics of Biomedicine Course . . . . . . . . 65

2.4 .1 Guest Lectures . . . . . . . . . . . . . . . 66

2.4.2 Use of Multimedia Modules in the Curriculum . . . . . . . . 66

2.4 .3 Laboratory Activities . . . . . . . . . . . . . . . . . 67

2.4.4 Student Presentations . . . . . . . . . . . . . 67

2.4.5 Pre/Post Content Quizzes . . . . . . . . . . . . . 68

$\begin{array}{lll}3 & \text { Research Methods } & 70\end{array}$

3.1 Description of Courses . . . . . . . . . . . . . . 70

3.1.1 Potential Confounding Factors . . . . . . . . . . . 71

3.2 Description of Student Cohort . . . . . . . . . . . . . . 73

3.3 Assessment Methods . . . . . . . . . . . . . . . . . . . 77

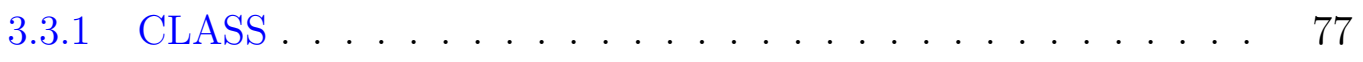

3.3 .2 Course Surveys . . . . . . . . . . . . . . . . . . 80

3.3 .3 Student Interviews $\ldots \ldots \ldots$

3.3.4 Illustration Conceptual Quizzes _ . . . . . . . . . . . 85

3.4 Quantitative Statistical Analysis . . . . . . . . . . . . . . . 89 
4 CLASS $\quad 91$

4.1 Fall and Winter Terms . . . . . . . . . . . . . . . . . . . 92

4.2 Spring Term . . . . . . . . . . . . . . . . . . 94

4.3 CLASS by Gender . . . . . . . . . . . . . . . . 97

4.4 Career-focused CLASS Questions . . . . . . . . . . . . . 98

4.5 Full Year Analysis . . . . . . . . . . . . . . . . . . . 103

4.6 Discussion . . . . . . . . . . . . . . . . . 105

5 Student Attitudes toward Physics 110

5.1 Response to the Course . . . . . . . . . . . . . . . . 111

5.2 Biomedical Expert Interviews _ . . . . . . . . . . . . . . . . 119

5.2.1 Connection to Medicine . . . . . . . . . . . . . 121

5.2.2 Actual Biomedical Experts . . . . . . . . . . . . . . 122

5.2 .3 Field-Specific Videos . . . . . . . . . . . . . . 125

5.2.4 Practical Relevance . . . . . . . . . . . . . 126

5.2.5 Viewership....................... 128

5.3 Relationship between Physics and Medicine . . . . . . . . . . . . 132

5.3.1 Categories . . . . . . . . . . . . . . . 132

5.3 .2 Survey Results . . . . . . . . . . . . . . . . 136

5.4 Should Physics be a Requirement? . . . . . . . . . . . . . . . . . 142

5.4.1 Full Year Analysis . . . . . . . . . . . . . . . . . 142

5.4 .2 Categories ..................... 144

5.4.3 Full Year Analysis with Categories . . . . . . . . . . . . . 147

5.4.4 Matched Data from Pre-health students . . . . . . . . . . . . 152

5.4.5 Value of Physics Concepts and Problem Solving Skills . . . . . 154

5.5 Discussion . . . . . . . . . . . . . . . . . 156 
6 Interview Analysis 158

6.1 Should spring term be required? - Students that agreed . . . . . . . 160

6.1 .1 Sarah . . . . . . . . . . . . . . . 160

6.1 .2 Allison . . . . . . . . . . . . . . . . . . . 164

6.1 .3 Hailey . . . . . . . . . . . . . . . . . . . . 167

6.1 .4 Leah . . . . . . . . . . . . . . . . . . . . . 169

6.1 .5 Emily . . . . . . . . . . . . . . . . . . 172

6.1 .6 Maria . . . . . . . . . . . . . . . . . 175

6.1 .7 Cathy . . . . . . . . . . . . . . . 176

6.2 Should spring term be required? - Students that were neutral _ . . 177

6.2 .1 Henry . . . . . . . . . . . . . . . . . . . . . 177

$6.2 .2 \quad$ Noah . . . . . . . . . . . . . . . . . . . . . 180

6.2 .3 April and Alan . . . . . . . . . . . . . . . . 184

6.3 Should spring term be required? - Students that disagreed . . . . . 186

6.3 .1 Ryan . . . . . . . . . . . . . . . . . . 186

$6.3 .2 \quad$ Alex . . . . . . . . . . . . . . . . . . . . 189

6.4 Discussion . . . . . . . . . . . . . . . . . . . . . . 193

7 Conceptual Understanding and Contextualization 195

7.1 Doppler Shift . . . . . . . . . . . . . . . . . . . . . 196

7.1 .1 Illustration Prompt . . . . . . . . . . . . . . . . 196

7.1.2 Conceptual Understanding . . . . . . . . . . . . . . . . 197

7.1.3 Real-World Contextualization . . . . . . . . . . . . . . 201

7.1 .4 Discussion . . . . . . . . . . . . . . . . . . . . 202

7.2 Total Internal Reflection . . . . . . . . . . . . . . . 203

7.2.1 Illustration Prompt . . . . . . . . . . . . . . . . . 203

7.2 .2 Conceptual Understanding . . . . . . . . . . . . . . . 206

7.2.3 Real-World Contextualization . . . . . . . . . . . . . . 211

7.2 .4 Discussion . . . . . . . . . . . . . . . . . . . . . 213 
7.3 Thin-Film Interference . . . . . . . . . . . . . . . . . . 215

7.3.1 Illustration Prompt . . . . . . . . . . . . . . . . . 215

7.3.2 Conceptual Understanding . . . . . . . . . . . . . . . . 218

7.3.3 Real-World Contextualization . . . . . . . . . . . . . . 224

7.3 .4 Discussion . . . . . . . . . . . . . . . . . 226

7.4 Image Formation by a Lens $\ldots \ldots \ldots \ldots \ldots \ldots$

7.4 .1 Illustration Prompt . . . . . . . . . . . . . . . . . 227

7.4.2 Conceptual Understanding . . . . . . . . . . . . . . . . . . 229

7.4.3 Real-World Contextualization . . . . . . . . . . . . . 236

7.4 .4 Discussion . . . . . . . . . . . . . . . . . . 237

7.5 Discussion . . . . . . . . . . . . . . . . . . . . . . 239

8 Discussion $\quad 242$

8.1 Research Question 1: Opinions on the Relevance of Physics . . . . . 243

8.2 Research Question 2: Conceptual Understanding and Contextualization 245

8.3 Implications for IPLS Research . . . . . . . . . . . . . . 246

8.3.1 Pre-health Students' Attitudes toward Physics . . . . . . . . 246

8.3.2 Utility Value of Physics to Life Science Students . . . . . . . 248

8.3.3 Use of the CLASS by IPLS Researchers . . . . . . . . . . . 249

8.3.4 Accounting for Gains in Favorable Attitudes . . . . . . . . . 250

8.3.5 Physics Content for Pre-Health Students . . . . . . . . . 252

8.3.6 Adoption by Instructors of Traditional Physics . . . . . . . . . 252

8.3.7 Influence of Peers and Professionals on Student Attitudes . . . 253

8.3.8 Viewership of Biomedical Expert Interviews . . . . . . . . . 254

9 Conclusion 256

$\begin{array}{ll}\text { Bibliography } & 260\end{array}$ 
A.1 School Year 2015-2016 . . . . . . . . . . . . . . . . . . 272

A.1.1 Full Year Trends . . . . . . . . . . . . . . . . . . . . 276

A.2 School Year 2014-2015 . . . . . . . . . . . . . . . . . 277

A.3 School Year 2013-2014 . . . . . . . . . . . . . . . . . . . 279

A.4 School Year 2012-2013 . . . . . . . . . . . . . . . . . . . . . 281

Appendix B Survey Questions 283

B.1 Pre Fall . . . . . . . . . . . . . . . . . . . . . . . . . 283

B.2 Post Fall . . . . . . . . . . . . . . . . . . . . 283

B.3 Post Winter . . . . . . . . . . . . . . . . . . . . . . . 285

B.4 Pre Spring . . . . . . . . . . . . . . . . . . . . . 287

B.5 Post Spring . . . . . . . . . . . . . . . . . . . . . . . . 289

$\begin{array}{ll}\text { Appendix C Illustration Quizzes Example } & 291\end{array}$

Appendix D Illustration Quiz Revisions 293

$\begin{array}{lll}\text { Appendix E Interview Protocol } & 297\end{array}$

$\begin{array}{lll}\text { Appendix F Interview Consent Form } & 300\end{array}$

Appendix G Institutional Review Board Approval 301 
List of Tables

2.1 Comparison of FFM and BF\% measurements by the custom BIA device and the OMRON. Coincidentally, both students had the same $\mathrm{BF} \%$ despite having different input parameters. 18

2.2 Summary of interviews with biomedical professionals. . . . . 50

2.3 Summary of chapters in the course text. . . . . . . . . . . 50

2.4 Overview of the topics covered in the multimedia modules . 58

2.5 Schedule of the spring 2014 laboratory section. . . . . . . . . . 60

2.6 Summary of student survey. In total 68 students submitted the survey. The number of students falling into a particular category is in parentheses. . . . . . . . . . . . . .

3.1 Student responses to the survey prompt"I identify my gender as...". . . . . . . . . . . . . . . .

3.2 Student responses to the survey prompt "What high school physics courses did you take?". . . . . . . . . . . . 76

3.3 Genders of interviewees and the students in the reformed

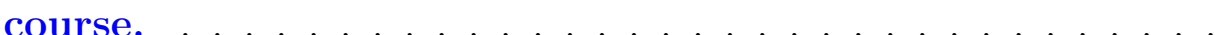

4.1 List of categories in the CLASS and the abbreviations used in this section.

4.2 Average percent of favorable responses from students in the reformed and traditional courses. Asterisks denote statistically significant shifts, which are defined as twice the standard error. Pre data came from the post winter survey and post data came from the post spring survey. The effect size (ES) for each category is also given. . . . . . . . . . . . . 
4.3 Statements from the Personal Interest and Real World Connection categories of the CLASS. Questions in italics are the rewritten versions of the questions, designed to emphasize students' careers. . . . . . . . . . . . . . . . . . .

5.1 Category definitions of student viewership of the biomedical expert interviews, where $d$ is the duration of the video and $t$ is the time from when a student first opens the video and the next submission to a question. . . . . . . . . . . . . .

5.2 Emergent categories of the responses to the prompt, "What is the relationship between physics and medicine?" . . . . . . 133

5.3 Distribution of the number of examples given by students in the pre and post spring survey about the relationship between physics and medicine. . . . . . . . . . . . . . . . . . . . . 141

5.4 Comparison of the number of students each term that responded to the Likert and open-ended (OE) questions about requiring physics courses. The percentages represent the fraction of students that replied to both formats. The percentage is calculated with respect to the number of students that replied to the Likert-style question. . . . . . . . . . . . . .

6.1 Students that participated in the interviews and their ratings of whether the course should be required. The students are sorted according to their rating of the spring term course. $(\mathrm{PT}=$ physical therapy, $\mathrm{PH}=$ pre-health, $\mathrm{NA}=$ no answer $)$

7.1 Students descriptions of the phase shift of the light ray in Fig. 7.12. . . . . . . . . . . . . . . 220

7.2 Distribution of students who drew correct solutions to the prompt in Fig. 7.20. The columns are separated by the location of the focal point of the lens. . . . . . . . . . . . . . . . . . 232 
List of Figures

2.1 Circuit models of the body: (a) $R_{e}$ is the resistance of ECW, $R_{i}$ is the resistance of $\mathrm{ICW}$, and $X_{C}$ is cellular membrane reactance; (b) For a single frequency, the effective resistance and reactance of the circuit in (a) can be found and the body can be modeled as a series RC circuit. . . . . . . . . . . . . . . 14

2.2 The custom built, educational BIA device and hand-held electrodes used in the laboratory activity. The electrodes consist of conducting tape, banana jacks, and plastic tubing. . . . . .

2.3 Correlation of FFM as measured by the OMRON and by the educational BIA device. Twenty-three men and nine women were tested.

2.4 Measurements of a series $\mathrm{RC}$ circuit using the educational BIA device $(R=470 \Omega$ and $C=10 \mathbf{n F})$ : (a) Impedance $Z$ vs. frequency; (b) Phase shift $\Delta \phi$ vs. frequency. . . . . . . . .

2.5 BIS measurements by the educational BIA device. (a) ColeCole plot of a parallel circuit as in Fig. 2.1(a) using $R_{e}=$ $470 \Omega, R_{i}=100 \Omega$, and $C=10 \mathrm{nF}$. (b) Cole-Cole plot of body impedance. Note that at the peak of the curve $f=50 \mathrm{kHz}$, which is the frequency normally used by SF-BIA. . . . . . .

2.6 Basic reconstruction technique of CT. (a) A light source and a detector are rotated around an area. The black boxes in the detector arrays indicate where the light is being blocked. (b) The projection data are spread back onto a grid along the original line. The overlapping sections reveal the contents of the area. . . . . . . . . . . . . . . . . . . . . . .

2.7 CT scanner with Vernier rotary motion sensor and photogate and an opaque enclosure made with a Congo Blue light filter. 
2.8 (a)The enclosure for the CT apparatus and the grid for securing the objects. (b)Positions of three cylinders inside the enclosure. The diameter of each cylinder is $0.6 \mathrm{~cm}$. The diameter of the enclosure is $7 \mathbf{c m}$. . . . . . . . . . . . . . . . 23

2.9 Geometry of the CT scanner. . . . . . . . . . . . .

2.10 (a) Scans of the cylinders as shown in figure 2.8(b) taken in $10^{\circ}$ increments over $=150^{\circ}$. The ring around the cylinders is caused by the opaque enclosure, which reflects the light from the photogate at very shallow angles effectively blocking it. (b) A completed scan taken in $10^{\circ}$ increments over $=360^{\circ}$. (c) The same data from (b) after applying a window. Note that the lower boundary of the grey scale has increased which obscures pixels with lower values . . . . . . . . . . . . .

2.11 (a) Model of the human eye used in the online lab and (b) a screenshot from an ISE that allows for the investigation of imaging with a lens . . . . . . . . . . . . . . . . 28

2.12 Screenshot of the online lab . . . . . . . . . . . . . . . 29

2.13 Radiography apparatus components: (a) infrared LED array, (b) enclosure mostly opaque to visible light but mostly transparent to IR, (c) slide with various attenuation coefficients, (d) objects concealed within the enclosure, and (e) detector screen emitting green light upon excitation by infrared light.

2.14 Arrangement for examination of attenuation effects on transmission. (a) Place the detector and slide on the base. (b) Place the lid on the base, hold the button, and view the image through the slot.

2.15 (a) Image of the analog up-converting phosphor detector and (b) digital image taken with a cellphone, displaying differences in image intensity corresponding to varying attenuation coefficients of materials in the slide. The left half (Congo Blue) of the slide is most intense, the right half (tissue paper) appears dim, and the bone (construction paper) appears dark as all transmission is blocked. . . . . . . . . . . . . 
2.16 Planar images of concealed objects similar to Fig. 1(d). (a) Experimental setup for the planar imaging activity. Figures (b) and (c) show how additional information about the objects can be attained by viewing from two angles. Figures (c) and (d) illustrate the differences between analog (phosphor screen) and digital (cellphone camera) detectors. . . . . . . .

2.17 (a) Apparatus used in the pulse oximetry lab activity. (b) Absorption spectra of the bromothymol blue solution as measured by a spectrometer. (Source Ref. [68]) . . . . . . . . 38

2.18 Fundamental functions of an EKG sensor. (Source Ref. [69]) 39

2.19 (a) A volunteer jumps from a small platform onto a force plate. (b) Force plate data of a volunteer jumping off a chair. The volunteer's weight is measured before the jump but not displayed (photo courtesy American Association of Physics Teachers).

2.20 Screen capture of the program used in the balance challenge. The graphs display the force plate data of three volunteers. Contestant 1 is balancing on two feet. Contestant 2 is balancing on one foot with open eyes. Contestant 3 is balancing on one foot with closed eyes. Note that at approximately 7.5 seconds Contestant 3 lost his balance and stepped off the force plate. . . . . . . . . . . . . . . . . . . . .

2.21 Trajectory of a ball attached to a helmet worn by the contestant for (a) a normal jump and (b) a jump in which the contestant tucks in his arms and legs. (c) By changing the position of his center of mass, the contestant's head follows a nonparabolic path. . . . . . . . . . . . .

2.22 Left panels An undergraduate pre-health student volunteer has his body composition measured and prepares to step into the Pod Bod, a device that measures body composition by air displacement. Right panels Screenshot of a video clip with a LASIK surgeon explaining the different types of lasers he utilizes during surgery. Schematic showing the cutting of the corneal flap with focused laser pulses. Image of the eye with open flap before reshaping of the cornea (courtesy Teplick Custom Vision). QR code of a video clip on LASIK. . . . . . 49

2.23 Sample course text from the chapter on LASIK . . . . . . . 51 
2.24 Sample pre-lecture question and number of trials students needed to get the correct answer . . . . . . . . . . . . 52

2.25 Sample checkpoint question and student responses . . . . . 54

2.26 Sample homework questions . . . . . . . . . . . . . . . 55

2.27 Student submissions to the endoscopy module. The plots show the number of students who submitted an answer (solid blue), the number of students who found the correct answer (dashed red), and the average number of submission per student (dotted green). . . . . . . . . . . . . .

2.28 Shifts of matched student CLASS responses between postwinter and post-spring term 2014. The error bars represent the standard error. The asterisks represent statistically significant data, i.e. a shift that is at least twice the standard error. The category abbreviations are listed in the methods section (Sec. 3.3.1). . . . . . . . . . . . . . .

3.1 Age distribution of students in the reformed morning course and the traditional evening course. . . . . . . . . . . 74

3.2 Class standing of the students in the reformed morning course and the traditional evening course. . . . . . . . . . . . 75

3.3 Planned professions of the students in the reformed and tra-

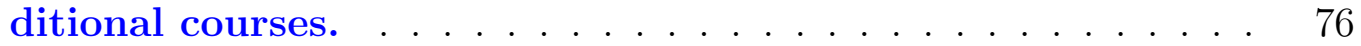

3.4 Class standing of the interviewees and the students in the reformed course. . . . . . . . . . . . . . . . 84

3.5 Planned professions of the interviewees and the students in

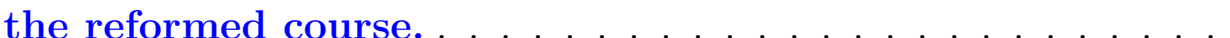

3.6 Illustration given to students for the quiz on total internal reflection. . . . . . . . . . . . . . . . .

3.7 Prompt for the illustrated conceptual quiz on total internal reflection.

4.1 Results of the CLASS administered at the beginning and end of fall term for students in (a) the morning section and (b) the evening section. The asterisks denote statistically significant shifts. . . . . . . . . . . . . . . . . . . . . . 
4.2 Results of the CLASS administered at the end of fall term and the end of winter term for students in (a) the morning section and (b) the evening section. The asterisks denote statistically significant shifts. . . . . . . . . . . . . .

4.3 Results of the CLASS administered at the end of the winter and spring terms for students in (a) the reformed course and (b) the traditional course. The asterisks denote statistically significant shifts. . . . . . . . . . . . . .

4.4 Results of the CLASS for women students in (a) the reformed course and (b) the traditional course. The asterisks denote statistically significant shifts. . . . . . . . . . . . .

4.5 Results of the CLASS for men students in (a) the reformed course and (b) the traditional course. The asterisks denote statistically significant shifts. . . . . . . . . . . . .

4.6 Results of the (a) modified and (b) original version of questions from the CLASS. 63 students from the reformed course (black squares) and 25 students from the traditional course (empty circles) responded to both. . . . . . . . . . . . .

4.7 Shifts in favorable and unfavorable responses from the (a) RWC and (b) PI categories of the CLASS for the full 20152016 school year. 46 students from the reformed course (black squares) and 22 students from the traditional course (empty circles) completed all four surveys. Green lines represent significant shifts. . . . . . . . . . . . . . .

5.1 Students responses to the post-course survey indicate that the course reforms were viewed favorably by the majority of students. The prompt was a five-point Likert-style question that ranged from strongly disagree to strongly agree. The results were condensed down to three points. . . . . . . . . 111

5.2 Students' ratings of how much time they spent studying as compared to previous terms. The prompt was a five-point Likert-style question that ranged from much more time to much less time. The results were condensed down to three

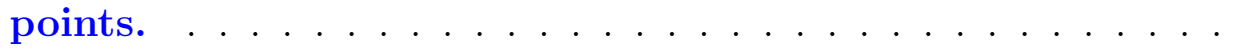

5.3 Ratings from matched students on how much time they spent on homework problems in (a) winter and (b) spring term. The prompt was a multiple choice question with the same intervals as shown in the graphs. . . . . . . . . . . . . . . . . 113 
5.4 Students ratings of how the biomedical applications have impacted their enjoyment and understanding of physics. The prompt was a five-point Likert-style question that ranged from strongly disagree to strongly agree. The results were condensed down to three points. . . . . . . . . . . . .

5.5 Results from the post winter and post spring survey. These results only include matched names of pre-health students who responded either (a) all three terms or (b) in winter and spring. Students are grouped by which course they took in spring term. Neutral responses are not plotted for clarity. The prompt was a five-point Likert-style question that ranged from strongly disagree to strongly agree. The results were condensed down to three points. . . . . . . . . . . . . .

5.6 Students' ratings of whether the fall term course should be reformed to focus on biomedical applications. These results only include matched names of pre-health students. The prompt was a five-point Likert-style question that ranged from strongly disagree to strongly agree. The results were condensed down to three points. . . . . . . . . . . . . .

5.7 Potential impact of biomedical expert videos as reported by students. This data only includes pre-health students. The prompts were five point Likert-style questions that ranged from strongly disagree to strongly agree. The results were condensed down to three points. . . . . . . . . . . . . . . 119

5.8 Students' ratings of the usefulness of different features of the reformed course. The prompts were five point Likert-style questions that ranged from extremely useful to useless. The results were condensed down to three points. . . . . . . . . . . 120

5.9 Students' ratings of how easy it is to engage with a physics concept when described by physicists versus biomedical experts. The prompt was a five-point Likert-style question that ranged from strongly disagree to strongly agree. The results were condensed down to three points. . . . . . . . . . 
5.10 Viewership of the biomedical expert interviews. Each point represents a different video in a given module. Viewership is based on logged click traffic. Viewers are defined as student who do not click on another link for at least $75 \%$ of the duration of the video but no longer than ten times the video duration. Others click another link before $75 \%$ of the video has played. . . . . . . . . . . . . . . . . .

5.11 Results from the pre spring survey for students in the reformed and traditional courses. Many students cited more than one example, therefore the percentages do not sum to one hundred . . . . . . . . . . . . . . . . . . . 137

5.12 Distribution of the number of examples of the relationship between physics and medicine in the pre spring survey. . . . 138

5.13 Results from the post spring survey for students in the reformed and traditional courses. . . . . . . . . . . . . . . . . 138

5.14 Distribution of the number of examples of the relationship between physics and medicine in the post spring survey. . .

5.15 Coded responses from matched pre-health students in the reformed and traditional courses about the relationship between physics and medicine. The results are from the (a) pre spring and (b) post spring surveys. . . . . . . . . . . . . . . . . . 140

5.16 Students' ratings of whether their physics course should be required. The number of students who answered the question and the total number of students registered are given for each course. The morning and evening sections are designated $\mathrm{AM}$ and $\mathrm{PM}$ respectively. The reformed course was the AM section of spring term. The prompt was a five-point Likertstyle question that ranged from strongly disagree to strongly agree. The results were condensed down to three points.

5.17 Students' explanations for their rating of whether physics should be a required course. Many students gave multiple examples and are placed in multiple categories. Therefore the percentages do not sum to one hundred. . . . . . . . . . . 148

5.18 Students' explanations for their rating of whether physics should be a required course after spring term. Many students gave multiple examples and are placed in multiple categories. Therefore, the percentages do no sum to one hundred. 
5.19 Students' ratings of whether their physics course should be required. These results only include matched names of prehealth students who responded either (a) all three terms or (b) in winter and spring. The students are sorted by which class they took in spring term. Neutral responses are not plotted for clarity. . . . . . . . . . . . . .

5.20 Students' explanations for their rating of whether physics should be a required course. The ratings are from (a) the post winter and (b) post spring surveys. The data includes only matched pre-health students. Some students gave multiple examples and are placed in multiple categories. Therefore the percentages do no sum to one hundred. . . . . . . . . . . . 153

5.21 Students' ratings of whether the problem solving skills they have learned in their course will be useful in their future career. These results only include matched names of pre-health students who responded either (a) all three terms or (b) in winter and spring. . . . . . . . . . . . . . . . .

5.22 Students' ratings of whether the concepts the have learning their physics course will be useful in their future career. These results only include matched names of pre-health students who responded either (a) all three terms or (b) in winter and spring. . . . . . . . . . . . . . . . .

6.1 Students' ratings of whether their physics course should be required from students in the reformed course and students that participated in the interviews.

7.1 Illustration given to students for the quiz on sound waves and Doppler shifts.

7.2 Distribution of the types of interactions between the sounds waves and the moving particle as depicted by students for the prompt in Fig. 7.1.

7.3 Distribution of students' contextualization of sound wave illustrated quiz prompt. Note that multiple students gave responses with more than one example. Therefore the category percentages do not sum to 100 percent. . . . . . . . . . . . 202

7.4 Distribution of the number of real-world application of the phenomena depicted in Fig. 7.1. . . . . . . . . . . . 202 
7.5 Illustration given to students for the quiz on total internal reflection. . . . . . . . . . . . . . . .

7.6 Illustration of the possible refraction and reflection events for the prompt in Fig. 7.5. The angle of reflection equals the angle of incidence $\theta_{1}$. Snell's Law states that the refracted ray will either bend toward or away from the normal depending on the values of $n_{1}$ and $n_{2}$. If $n_{1}<n_{2}$ and the incident angle is greater than the critical angle, all of the light will reflect and no light will refract. The angles are taken with respect to the normal. . . . . . . . . . . . . . . . . . . . 205

7.7 Distribution of events drawn by students to the prompt in Fig. 7.5.

7.8 Distribution of refraction events drawn by students to the prompt in Fig. 7.5. . . . . . . . . . . . . . . . . 208

7.9 Distribution of the angles drawn by students to the prompt in Fig. 7.5. The category 'only normal given' represents drawings where the normal was included but no indication of the location of the angle. The 'incident' represents angles with respect to the path of the incident ray in the absence of the $n_{2}$ medium. $\ldots \ldots \ldots \ldots \ldots \ldots$

7.10 Distribution of students' real-world applications of the physical concept depicted in Fig. 7.5. Many students gave multiple examples, so the percentages do not sum to 100 percent.

7.11 Distribution of the number of real-world application of the phenomena depicted in Fig. 7.5. . . . . . . . . . . . . . . 212

7.12 Illustration given to students for the quiz on thin-film interference. . . . . . . . . . . . . . . . . . 216

7.13 Customary depiction of thin-film interference. . . . . . . . 217

7.14 Distribution of students' rankings of the refractive indexes for Fig. $7.12 \ldots \ldots \ldots \ldots \ldots \ldots \ldots \ldots$

7.15 Locations of the surfaces used in the analysis of reflection and

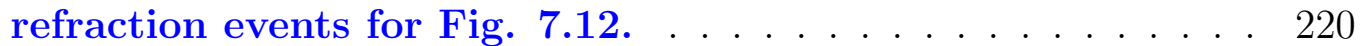

7.16 Distribution of students' depictions of refraction events for Fig. 7.12. The location of the three surfaces are shown in Fig. 7.15. . . . . . . . . . . . . . . . . . . 222 
7.17 Distribution of students' depictions of refraction events from the TIR quiz (Sec. 7.2). Only students from the 'no bend' category from Fig. 7.16(a) are included here. . . . . . . . . . 224

7.18 Distribution of students' real-world applications of the concept depicted in Fig. 7.5. . . . . . . . . . . . . . . . . . 225

7.19 Distribution of the number of real-world applications of the concept depicted in Fig. 7.5. . . . . . . . . . . . . . . . . 225

7.20 Illustration given to students for the quiz on image formation by lenses. . . . . . . . . . . . . . . . . . . . . 228

7.21 Possible correct answers to the prompt in Fig. 7.20. The paths of the rays exiting the lens depend of the location of the point source to the focal point $(f)$. (a) If the point source is located at the focal point, the rays will exit parallel to the optical axis. (b) If the point source is located farther than the focal point, the rays will converge. (c) If the source is located in between the focal point and the lens, the rays will diverge. Note that each image uses a different scale.

7.22 Distribution of the locations of focal points as drawn by students. . . . . . . . . . . . . . . . 230

7.23 Example drawing by a student. The focal points of the lens are drawn on both sides. The student incorrectly depicts light from the focal point on one side converging at the focal point on the other side of the lens.

7.24 Example drawing by a student. The focal point is drawn only on the right side of the lens. The converging rays are incorrectly shown as passing through the focal point. . . . . . 232

7.25 Comparison of the paths of light rays as drawn by students who did or did not include focal lengths. . . . . . . . . . . 233

7.26 Comparison of the paths of light rays as drawn by students in the reformed and traditional courses. . . . . . . . . . . . 234

7.27 Examples of the location of the lens refraction as drawn by students. The drawings shoe the refraction occurring at (a) both sides of the lens, (b) the principal plane, and (c) the left side of the lens only. 
7.28 Distribution of students' depiction of the location of the lens refraction. Chi-square test of independence resulted in a significant difference in the distributions. $\chi^{2}(6, N=150)=$ $20.3, p<0.01 \ldots \ldots \ldots \ldots \ldots \ldots \ldots \ldots \ldots \ldots$

7.29 Distribution of students' real-world contextualization of the image formation prompt (Fig. 7.20). Many students gave multiple examples, so the percentages do not sum to 100 percent. . . . . . . . . . . . . . . . . . . . . 236

7.30 Distribution of the number of real-world applications given by students of the image formation prompt (Fig. 7.20). . . . 236 


\section{Introduction and Background Information}

\subsection{Introductory Physics for Pre-health and Life Science Students}

In recent years, several publications and expert panel reports from members of the higher education community have raised concerns about the relevance of science content in introductory physics for the life sciences (IPLS) curricula [1-5]. Pre-medical education has been perceived to be neither coherent nor well-structured [4]. The impact of physics instruction on student attitudes has been notoriously poor for introductory courses [6-9]. It has been argued that science content in current pre-medical curriculum needs revisions to improve the education of future healthcare professionals on relevant science competencies, skills, and learning habits [10]. Experts on biology education call for an interdisciplinary, outcome-oriented, and inquiry-driven curriculum [11-14]. These concerns are increasingly important given the unprecedented rate of advancements in both medical science and technology during the last 50 years $[2,15]$. There is agreement among leading biology and medical researchers that future medical students need to become much more knowledgeable in basic physics, chemistry, and mathematics $[8,10]$.

Though many algebra-based introductory physics courses are dominated by prehealth and life science students, a focus on practical and emerging biomedical applications is not typical. Physics instructors often lack solid knowledge of the biomedical 
field and thus are not able to make the physics concepts they teach relevant to these students. On the other hand, a solid understanding of the physics behind modern biomedical technologies is valuable for the healthcare professional workforce to choose the most appropriate diagnostic and treatment modalities that improve the quality of patient care while minimizing extraneous medical costs or adverse health effects to the patient $[16,17]$.

Students applying to healthcare programs must often earn high marks on entrance exams like the Medical College Admission Test (MCAT), which contains a physical sciences section. Released in 2015, the revised MCAT has moved toward a competency-based interdisciplinary evaluation of students' abilities to merge what they have learned and apply it within a biological context [18-20].

In light of these trends, many colleges and universities have begun to reform their curriculum to better serve students aiming for a career in biology and the health sciences. Laboratory exercises are being developed that explore and elucidate the physics underlying biological processes and medical technologies [21-27]. New and reformed courses are being offered that teach physics concepts within a biological or medical context [28-34]. There are an increasing number of physics textbooks that can be used to accompany similar courses [35-42]. In addition, there is a growing community of instructors and researchers specializing in the development, assessment, and dissemination of these materials [43]. The increased interest in and support for reforming physics education for pre-health students is an excellent opportunity for effecting significant change. 


\subsection{Physics for Pre-Health Students at PSU}

As with many other universities, the algebra based introductory physics courses at Portland State University (PSU) have a large number of pre-health students. In the past, the algebra based course followed a traditional format with little connection to the health sciences. Results of surveys showed that students in these courses experienced the same frustrations as their peers at other colleges and universities. Pre-health students at PSU expressed dissatisfaction about being required to take a physics course that they feel has little to do with their academic and professional careers.

Starting in 2008, PSU began offering a summer-term, intermediate-level elective Physics in Biomedicine course [44]. The course explores the many ways in which physics and the health sciences intersect. This connection is reinforced through lectures and laboratory activities that focus on medical devices and technologies as well as guest lectures from local biomedical experts. Experiences from this course and the need for reformed physics courses for pre-health students led to the development of a one-term introductory physics course offered first spring term 2014 [45]. It is the third term in a three-term sequence and focuses on waves and optics. This course follows the "flipped-classroom" model, which encourages active learning during class time through activities like group work rather than exclusive, up-front lectures. Another important aspect of the course is the inclusion of many multimedia instructional elements. Each module in the course begins with videos of interviews with biomedical experts, which the students watch before coming to class. [46] The experts detail how the physics content of the module is important in their field. A course text and accompanying homework problems were written for the course and are presented to students through an online platform. 
The research work for this dissertation is the development, implementation, and assessment of curriculum for the introductory and intermediate level physics courses at PSU that targets the needs of pre-health students. This curriculum includes laboratory activities, multimedia content, and other instructional materials all of which present physics content in biomedical contexts. The curriculum covers a wide range of physical topics including waves and optics as well as nuclear and atomic physics.

The multifaceted assessments of the curriculum probe both the learning efficacy as well as the attitudinal effect they may have had on students. Students' attitudes toward both traditional and reformed physics instruction is explored through multiple tools including surveys, interviews, and conceptual quizzes.

\subsection{The Role of Interest in Learning}

The affective domain is widely viewed as an important aspect of learning [47-56]. It consists of a number of constructs that deal with students' perceptions and beliefs. Of these many components, Koballa states that, "Attitude and motivation are indeed the most critically important constructs of the affective domain in science education" [53]. Attitude can be generally defined as the inclination to react favorably or unfavorably to an idea, whereas motivation is the internal condition that dictates behavior $[53,56]$. One of the significant factors that can shape attitude and motivation is interest, which is known to be influential in student achievement, cognition, and future academic decisions $[47,48,50,56]$.

Interest development can be divided into multiple stages including situational and individual interest $[47,50,55,56]$. Situational interest is the first stage of interest development and occurs when the learner is introduced to an idea through some ex- 
ternal influence such as course instruction. While situational interest can lead to beneficial effects such as focusing attention and assimilating prior and new ideas, it does not necessarily persist over time [50]. Individual interest is a deeper connection to the idea and persists without external motivation. If the learner is to develop from situational to individual interest, it is necessary that they see value in the tasks that foster the initial situational interest $[47,50,55,56]$. Students may perceive a valuable learning task to be of either utility value (important outside of the immediate situation) or intrinsic (enjoyable or meaningful) [48]. For example, if a student perceives instruction to be an important part of his or her professional training, that instruction has utility value.

\subsection{The Role of Interest in Introductory Physics for the Life Sciences}

Pre-health students that enroll in physics courses often do so as a major requirement. It is common for these students to report that they do not view the content of the course to be important in their future careers [33,57-59]. These students show a lower level of personal interest in the course material than students who can more clearly see the benefit of learning physics (e.g. engineering students) [60]. Education research suggests that one of the necessary steps in developing individual interest is that students must perceive that the instruction has utility value $[48,49]$.

Summary of other utility value studies.

Attitudinal studies of life-science students enrolled in physics courses suggest that traditional instruction is not adequately demonstrating utility value [28, 33, 60, 61].

IPLS courses specifically address the utility value of physics education for prehealth and life science students by incorporating medical and biological concepts. 
The reforms that demonstrate this value are specifically based on the needs of the students. To identify those needs, researchers rely on professional reports from the health and life sciences, collaborations with educators within the students' field of interest, and what abilities are needed to excel in qualifying exams such as the MCAT $[6,10,19,28,33,58,62]$. Special attention is also paid to ensuring that the context in which the physics is presented is viewed as authentic [6,62], which promotes a stronger connection to the material and a deeper level of interest.

\subsection{Research Questions}

IPLS researchers stress that courses should be designed to best serve the needs and

goals of the students $[6,28,58]$. For many of the IPLS courses that have been developed at other universities, this has meant framing the physics content within a context relevant to biology and the life sciences $[28,29,58]$. The students that enroll in the algebra based introductory physics courses at PSU are predominantly pursuing a career in the health sciences. The reformed IPLS course at PSU is therefore designed to frame physical concepts in a biomedical context.

The purpose of this research is to determine students' attitudes toward the reformed algebra-based introductory physics course. This course emphasizes the role of physics in biomedicine by teaching the fundamental physical concepts underlying medical technologies.

The hypothesis explored in this research is:

By participating in the reformed course, pre-health students will exhibit increased levels of interest in physics and be able to accurately describe physical concepts in a biomedical context. 
This hypothesis can be broken down into individual research questions.

1. Are students' opinions on the relevance of physics impacted by biomedicallyfocused physics instruction?

2. Do students learn core physics principles and make links to biomedical applications from the curriculum?

Assessing pre-health students' interest in physics and their perception of its relevance to biomedicine was the primary goal of this research. We measured shifts in these areas through the Colorado Learning Attitudes about Science Survey (CLASS) [60], original attitudinal questions designed specifically for this course, and interviews with students that explore the themes seen in the surveys. It should be emphasized that although the goal of the course reforms was to increase students' interest in physics by highlighting its relevance to students' future careers, we did not intend to convey to students that every aspect of physics instruction would be directly beneficial. For example, physics courses entail a large amount of calculations that are unlikely to transfer to the day-to-day tasks of medical professionals. Rather, the goal was to engage students in physics by contextualizing it in a field they are already interested in.

The second research question addressed whether there was a difference in students' conceptual understanding and contextualization of the physics content. Students in both the traditional and reformed courses were assessed through open-ended prompts concerning diagrams often encountered in physics courses. These quizzes were analyzed for conceptual understanding and real-world contextualization. Specifically, we explored whether students in the reformed course used biomedical contexts for answering the prompts more often than students in the traditional course. 


\subsection{Structural Overview}

Chapter two describes the many course reforms developed for this project and their implementation in the classroom. Multiple laboratory activities have been developed as part of the physics in medicine project. They explore the physical concepts behind ubiquitous medical devices such as computed tomography scanners and body composition analyzers. The multimedia content used in the course is discussed as well. This includes video interviews with biomedical experts, course text, and medically relevant homework questions. The material is presented online through FlipItPhysics [63], which allows for computational analysis of student performance. Components of the curriculum have been used in both the introductory physics and an intermediate level physics course at PSU. Descriptions of these courses and how they incorporate the biomedical content is also discussed.

Chapter three discusses the research methods used in the study. The structure of the reformed and traditional courses is discussed as well as potential confounding factors that could have impacted the study. Descriptions of each of the assessment tools are given. These include the Colorado Learning Attitudes about Science Survey (CLASS), course surveys, interviews, and illustration-based conceptual quizzes. A brief explanation for the statistical tools used in the analysis is also provided.

Chapter four explores the results of the CLASS. Results from the entire year are discussed, with an emphasis on shifts in attitudes over spring term. The ability of the CLASS to probe shifts in students' attitudes toward the connection between physics and their future careers is discussed in this chapter as well.

Chapter five contains analysis of the course surveys administered over the course

of the whole year. The first section of this chapter describes the response from the 
students that took the reformed course in spring term. The next section explores the opinions of those same students toward the biomedical expert interviews. The final two sections deeply explore students' responses to two survey questions given multiple times throughout the year:

1. What do you view as the relationship between physics and medicine?

2. Do you think physics should be required for your major or graduate program?

Chapter six is a detailed analysis of the results of the student interviews. Students are organized based on their response to the prompt asking them whether they feel that physics should be required. Many of the trends that were observed in chapter six reappear in the interviews, which provide a more in-depth observation of students' attitudes.

Chapter seven describes the results of the illustration-based conceptual quizzes. Each of the four quizzes will be analyzed for students' conceptual understanding and real-world contextualization of the physical phenomenon. Comparisons are also made between the traditional and reformed courses.

Chapter eight will discuss the results of the study and their implications concerning the hypothesis and research questions. Recommendations for future research both at PSU and for the IPLS community will be discussed as well.

Chapters nine and ten contain the conclusion and appendix respectively. Components of the appendix include the history of CLASS results at PSU, the complete list of survey questions given during the 2015-2016 school year, and the interview protocol. 


\section{Descriptions of Course Reforms}

This chapter details the curricula developed at PSU as part of the reforms of physics instruction for pre-health students. Multiple laboratory activities were created on a variety of topics. These labs have been used in both the traditional and reformed versions of the introductory physics sequence as well as the intermediate-level course. The multimedia material features text, homework problems, and biomedical expert interviews. This material has been used in both the introductory and intermediatelevel courses. Descriptions of the structure and content of these courses will also be given in this chapter.

The laboratory activities and the multimedia modules were assessed using pre/post content quizzes. These quizzes were created by first identifying the desired learning goals and formulating questions to probe students' understanding. These pre/post quizzes have been used in conjunction with student feedback to improve the content. Multiple student researchers were also involved in the creation and analysis of the pre/post quizzes over the years. Although an example of a pre/post quiz question will be presented later, the full results of these quizzes are beyond the scope of this dissertation.

In addition to the pre/post quizzes, each of the multimedia modules described in this chapter have also been analyzed using students' log data. The FliItPhysics framework records every submission each student makes to every question [63]. As part 
of this project, I have created extensive python code to analyze this data. Variables that were analyzed include the correctness of students' responses and guessing habits seen in multiple choice questions. Based on the results of this analysis, the team made multiple revisions to the multimedia modules for improved clarity. Examples of the analysis will be described later in this chapter; however detailed descriptions are beyond the scope of this dissertation.

\subsection{Laboratories}

Multiple laboratory activities that focus on exploring the physical principles behind common medical devices or concepts have been developed for this project. All of the activities in this chapter have been used at PSU in either the traditional introductory physics course, the reformed introductory course (see chapter 2.3), or the intermediate level Physics of Biomedicine course (see chapter 2.4). More information can be found at the project webpage [64].

I was the lead developer of the bioelectrical impedance analysis (BIA) [65] and computed tomography (CT) labs [66]. These labs will be discussed in greater detail than other activities in this section.

The laboratory activity on the Optics of the Human Eye was created at Heinrich Heine University (HHU) in Germany. PSU, HHU, and the University DuisbergEssen (UDE) collaborated to translate the modules into English from the original German, transfer them for use in the introductory physics course at PSU, and study the effectiveness of the transfer. While I was not the lead investigator of the study, I translated the online material and carried out the assessment conducted at PSU [67]. Furthermore, the labs were part of the required laboratory session of the 2014 and 
2016 versions of the reformed introductory courses. Therefore, a brief description of the labs will be provided.

I advised an undergraduate student researcher during the development of the radiography activity. I was not directly involved in the development of the Pulse Oximetry [68] or EKG labs [69], and they are therefore not described in detail here. However, since I was involved in the implementation and assessment of the labs and because they are all facets of the overall project, a brief description of each has been included here.

The kinesthetic activities [70] described at the end of this section have been used in the fall term of the introductory physics courses. Unlike the other labs discussed in this chapter, these activities were used as demonstrations during the lecture rather than in the laboratory sessions. I was one of the three lead developers of these activities and wrote the majority of the computer code that collected, analyzed, and reported the data in real time. 


\subsubsection{Bioelectrical Impedance Analysis}

By completing this lab, students are exposed to principles of $\mathrm{RC}$ circuits by connecting medical inquiry with the function of a common device. Students practice circuit analysis by relating body impedance to simple electrical components. Outcomes range from an increased understanding of concepts needed for a solid physics background for Science, Technology, Engineering, and Mathematics (STEM) majors to an integrated, real-world appreciation for the necessity of that understanding. The complexity of the BIA laboratory is at an introductory physics level, but can be expanded to challenge those in upper division courses. Supplemental information on this activity can found in reference [65].

\section{Body Composition}

One of the uses of BIA is to calculate the fat-free mass (FFM), or lean mass, of a person based on measurements of the electrical characteristics of the body and empirical data. There are two main electrical properties that characterize body tissues, resistance and capacitance. Cell membranes conducting an electrical current behave similar to capacitors $[71,72]$. Body fluids are good conductors due to their ionic nature, while fat cells are not [73]. Bone is also considered a non-conductor under typical BIA conditions [74]. The resistive measurement of BIA, therefore, relates only to soft tissue hydration.

Total body water (TBW) can be broken down into extracellular water (ECW) and intracellular water (ICW), both of which have resistive electrical properties. Since cell membranes have a capacitive nature, body impedance is frequency dependent. Each of these components can be accounted for in a simplified circuit model of the 
body 2.1(a) [72]. At low frequencies, current flows primarily through ECW because cell membranes are essentially nonconductive. At higher frequencies, the reactance of cell membranes drops and current traverses ICW as well as ECW. Once these components are obtained, lean tissue mass can be calculated because physiological constants relate the two [71]. These correlations are based on large samplings of healthy individuals [75].

\section{Single Frequency BIA}

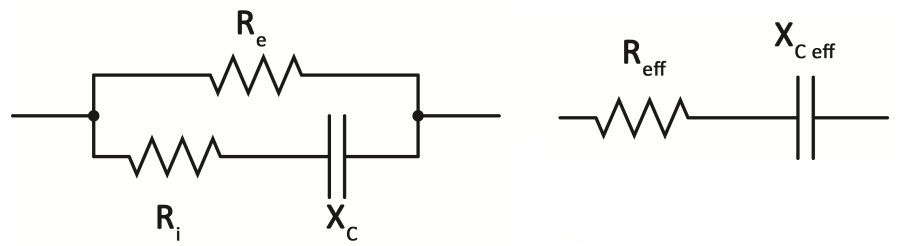

(a)

(b)

Figure 2.1: Circuit models of the body: (a) $R_{e}$ is the resistance of ECW, $R_{i}$ is the resistance of $\mathrm{ICW}$, and $X_{C}$ is cellular membrane reactance; (b) For a single frequency, the effective resistance and reactance of the circuit in (a) can be found and the body can be modeled as a series RC circuit.

The most validated method of obtaining TBW is single frequency BIA (SF-BIA) [71]. At a single frequency, the body can be represented as a simple circuit with a resistor representing the effective resistance of TBW in series with a capacitor representing cell membranes, as shown in Fig. 2.1(b). Body impedance is typically measured at $50 \mathrm{kHz}$ and empirical relationships are used to derive FFM from the measured effective resistance [75]. As a first approximation, one can consider a body impedance measurement from hand-to-hand as having a cylindrical geometry, therefore SF-BIA equations are based on the relationship between the resistance $R$, resistivity $\rho$, and the dimensions of a cylindrical object. A fully empirical relationship for FFM includes extra terms dependent on weight, age, gender, electrode placement, and other factors. 


\section{Design of the Educational BIA Device}

BIA measurements are taken by injecting a small alternating current (AC) into the body. By comparing the potential across the body to one across a known reference resistor, it is possible to determine the equivalent electrical components that model the body. To explore the process by which AC current is used in BIA, we designed our own battery powered educational BIA device, which is shown in Fig. 2.2.

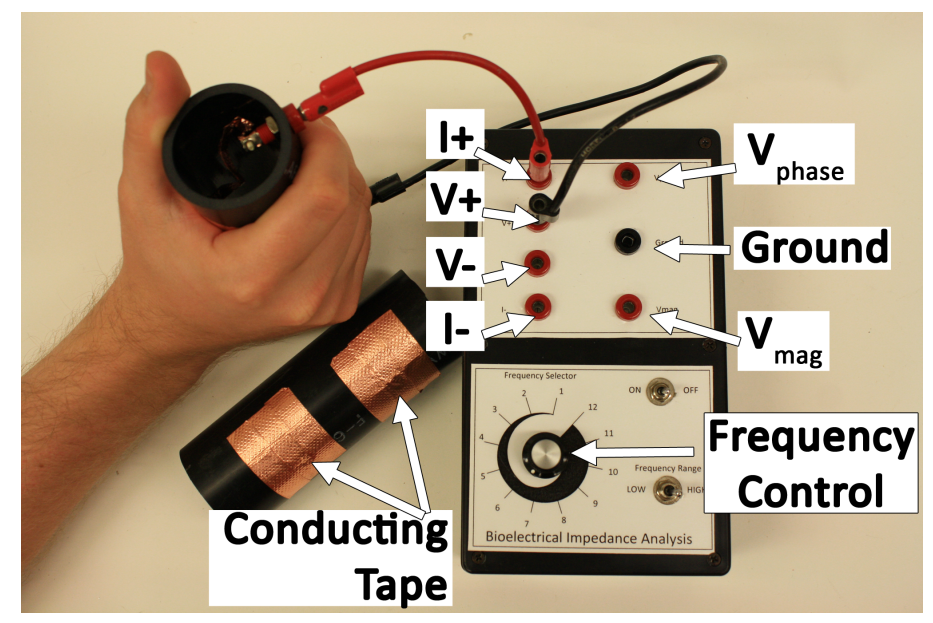

Figure 2.2: The custom built, educational BIA device and hand-held electrodes used in the laboratory activity. The electrodes consist of conducting tape, banana jacks, and plastic tubing.

The BIA device is based around the AD8302 Gain and Phase Detector, which can be used to find the impedance and phase of a circuit containing a combination of resistors and capacitors ( $\mathrm{RC}$ circuit) [76] and has already been shown to work in a BIA device [77]. The Gain and Phase Detector takes two sinusoidal signals as inputs and outputs two DC potentials, $V_{\text {phase }}$ and $V_{\text {mag }}$. Here $V_{\text {phase }}$ is proportional to the phase shift $\Delta \phi$ between the inputs, and $V_{m a g}$ is proportional to the logarithm of the ratio of the connected impedance $Z$ to the reference resistance $R_{\text {ref }}$. Thus, we have $V_{\text {phase }} \propto \Delta \phi$ and $V_{\text {mag }} \propto \log _{10} \frac{Z}{R_{\text {ref }}}$. (See Ref. [76] for the full conversion formulas.) Using these measurements, the resistance and capacitance of the test circuit can be 
found using formulas for impedance and phase shift of circuits like those in Fig. 2.1.

A common measure of body composition is the body fat percentage (BF\%) defined as

$$
B F \%=100 \% \times \text { fat mass } / \text { total mass } .
$$

To find an accurate equation for BF\%, including appropriate fitting factors, as measured by the educational BIA device, we surveyed 32 volunteers who provided their height, age, and gender. The participants' weights were measured using a force plate. Impedance and phase information was obtained using the BIA device. An estimate for FFM for each participant was found using the collected data and a published BIA equation for FFM. The participant's FFM was also measured with a commercially manufactured hand-to-hand BIA device, the OMRON HBF-306C [78], which acted as our reference. The measurements from the OMRON were plotted versus the published equation, and the slope and the offset from the linear fit of this plot were used to modify the published equation to yield a new empirical equation for FFM based on the impedance and phase measurements from the educational BIA device. The resulting equation is

$F F M=0.360 \cdot \frac{\text { Height }^{2}}{R}+0.162 \cdot$ Height $+0.289 \cdot$ Weight $-0.134 \cdot$ Age $+4.83 \cdot$ Gender -6.83,

where FFM is measured in $\mathrm{kg}$, height in $\mathrm{cm}$, weight in $\mathrm{kg}$, age in years, and gender is either 1 for men or 0 for women. This modified FFM equations had good agreement with the OMRON device, as shown in Fig. 2.3.

\section{Laboratory Exercises}

The major goals of the laboratory exercises are to develop a foundational understanding of how resistors and capacitors behave in $\mathrm{AC}$ circuits, to demonstrate how the 


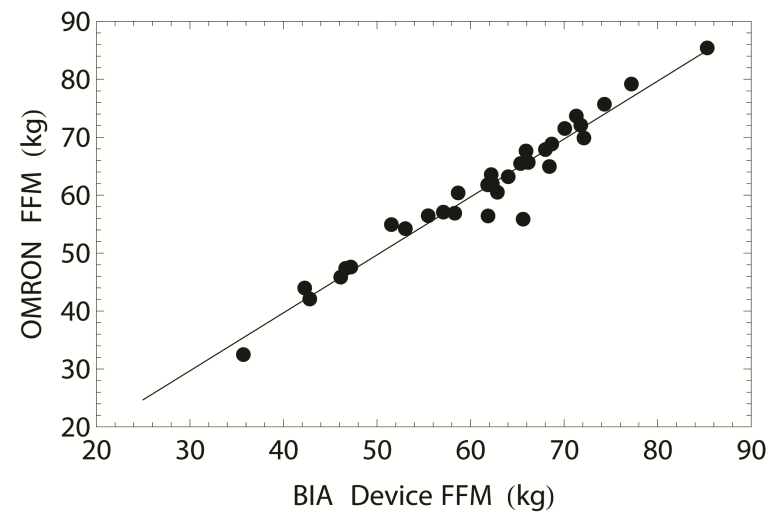

Figure 2.3: Correlation of FFM as measured by the OMRON and by the educational BIA device. Twenty-three men and nine women were tested.

body can be modeled as an RC circuit, and to show how the electrical characteristics of the body are used to elicit physiological information. Specifically, students explore the frequency dependence of resistance, reactance, impedance, and phase angle in RC circuits using tools like phasor diagrams or, for more advanced students, complex analysis of impedances. These concepts are introduced and demonstrated with a series RC circuit then applied to impedance measurements of student volunteers.

Simple RC Circuit During the first part of the laboratory, experimentation with a series RC circuit is undertaken to confirm the frequency dependence of phase angle and impedance. Students analyze the potentials across circuit components when a resistor and capacitor are connected in series to the educational BIA device. Students plot the experimental and theoretical values for impedance and phase shift versus frequency (Fig. 2.4). This part of the laboratory explores many of the key concepts of $\mathrm{AC}$ circuits taught in introductory physics and helps students get familiar with the educational BIA device.

Single Frequency BIA of the Human Body In this section, students use the educational BIA device and the empirical fit given in Eq. 2.2 to measure the body 

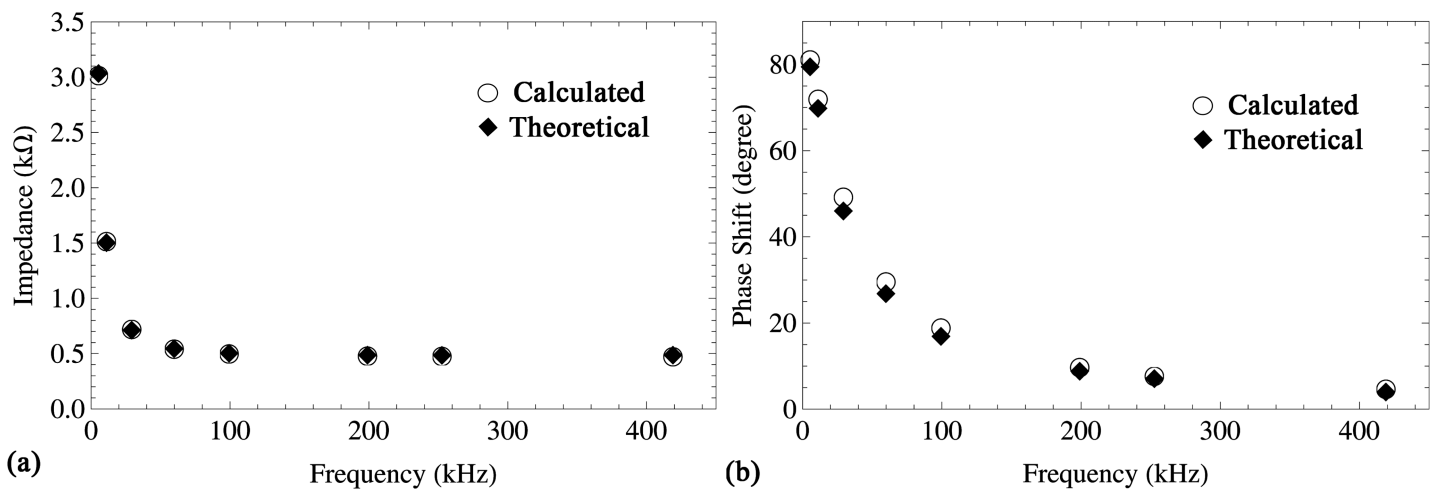

Figure 2.4: Measurements of a series RC circuit using the educational BIA device ( $R=470 \Omega$ and $C=10 \mathbf{n F}$ ): (a) Impedance $Z$ vs. frequency; (b) Phase shift $\Delta \phi$ vs. frequency.

impedance of a volunteer. Students measure the volunteer's $V_{\text {phase }}$ and $V_{\text {mag }}$ at a frequency of $50 \mathrm{kHz}$ and calculate resistance and capacitance for the single-frequency series-equivalent circuit of Fig. 2.1(b). The student enters the value for $R$ obtained with the educational BIA device, along with the height, weight, age, and gender into Eq. 2.2. The OMRON BIA device (which also operates at $50 \mathrm{kHz}$ ) is then used to measure $\mathrm{BF} \%$ for the volunteering student, and the two values are compared (See the results in Table 2.1).

Table 2.1: Comparison of FFM and BF\% measurements by the custom BIA device and the OMRON. Coincidentally, both students had the same BF\% despite having different input parameters.

\begin{tabular}{|c|c|c|c|c|c|c|c|c|c|c|c|}
\hline \multirow[b]{2}{*}{ Student } & \multirow[b]{2}{*}{$\begin{array}{l}f \\
(\mathrm{kHz})\end{array}$} & \multirow[b]{2}{*}{$\begin{array}{l}V_{\text {phase }} \\
\text { (V) }\end{array}$} & \multirow[b]{2}{*}{$\begin{array}{l}V_{\text {mag }} \\
(\mathrm{V})\end{array}$} & \multirow[b]{2}{*}{$\begin{array}{l}Z \\
(\Omega)\end{array}$} & \multirow[b]{2}{*}{$\begin{array}{l}\Delta \phi \\
(\mathrm{deg})\end{array}$} & \multirow[b]{2}{*}{$\begin{array}{l}R_{e f f} \\
(\Omega)\end{array}$} & \multirow[b]{2}{*}{$\begin{array}{l}x_{c_{e f f}} \\
(\Omega)\end{array}$} & \multicolumn{2}{|c|}{ BIA Device } & \multicolumn{2}{|c|}{ OMRON } \\
\hline & & & & & & & & $\begin{array}{l}\text { FFM } \\
(\mathrm{kg})\end{array}$ & BF\% & $\begin{array}{l}\text { FFM } \\
(\mathrm{kg})\end{array}$ & BF\% \\
\hline $\mathrm{A}^{a}$ & 50.4 & 1.836 & 1.040 & 685.5 & 4.7 & 683.2 & 56.5 & 62.8 & 24.7 & 63.8 & 23.5 \\
\hline $\mathrm{B}^{b}$ & 50.4 & 1.857 & 1.081 & 800.3 & 2.8 & 799.3 & 39.3 & 45.1 & 24.7 & 46.8 & 23.6 \\
\hline
\end{tabular}

Multi-frequency BIA and bioelectrical impedance spectroscopy In this section, students measure the effective resistance and reactance of the series equivalent circuit of Fig. 2.1(a) over a range of frequencies. These values are graphed with reac- 
tance on the $\mathrm{y}$-axis and resistance on the $\mathrm{x}$-axis; such a graph is often referred to as a Cole-Cole plot $[71,79]$. The result is a semi-circular curve whose center is depressed below the x-axis (Fig. 2.5). The resistances $R_{0}$ and $R_{\infty}$, the low- and high-frequency extremes, are the intersections of the curve with the x-axis. In bioelectrical impedance spectroscopy (BIS), these resistances are used to calculate ECW and ICW [75]. The peak of the semicircle for an average human body occurs near $50 \mathrm{kHz}$ [72]. At this frequency, current passes through both ICW and ECW.

Next, a student volunteer repeats the set of MF-BIA measurements with the handheld electrodes. Using the measured resistances and reactances of the human body, a new Cole-Cole plot is constructed, as shown in Fig. 2.5(b). Students compare the salient features of this plot with that of the parallel circuit. The peak of the semi-circular arc for the human body is near $50 \mathrm{kHz}$, thus indicating to students why SF-BIA uses that frequency.
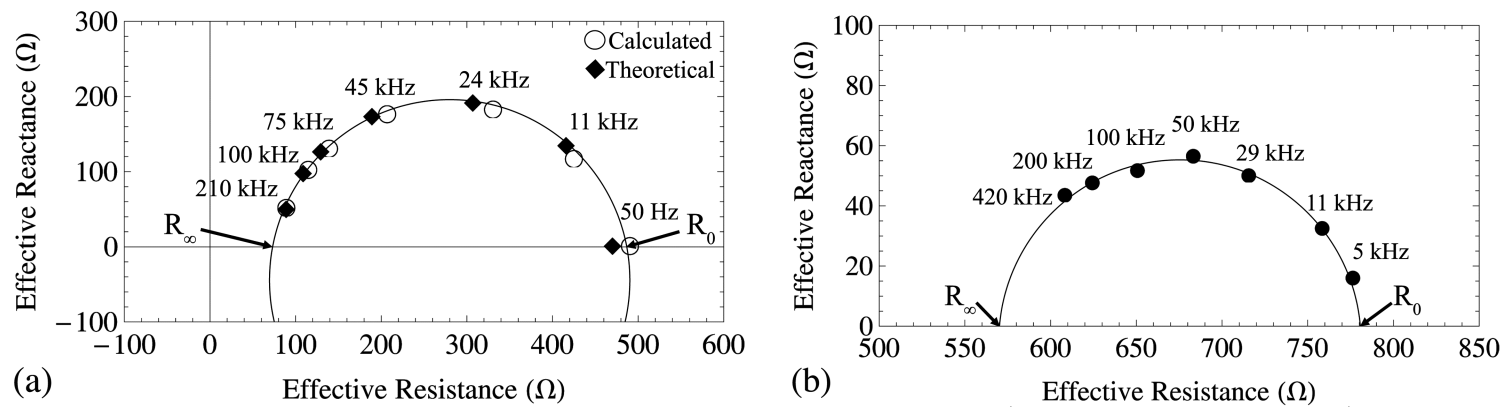

Figure 2.5: BIS measurements by the educational BIA device. (a) Cole-Cole plot of a parallel circuit as in Fig. 2.1(a) using $R_{e}=470 \Omega, R_{i}=100 \Omega$, and $C=10 \mathbf{n F}$. (b) Cole-Cole plot of body impedance. Note that at the peak of the curve $f=50 \mathrm{kHz}$, which is the frequency normally used by SF-BIA. 


\section{Learning Goals}

After completing the lab activity, students should be able to explain a number of concepts related to $\mathrm{RC}$ circuits and $\mathrm{AC}$ voltages as well as why these topics are important in biomedicine. The specific learning goals are:

1. Students will be able to explain how resistors and capacitors behave in AC circuits in terms of the frequency dependence of current, potential, and impedance.

2. Students will be able to explain the relationships between resistance, reactance, impedance, and phase angle.

3. Students will be able to explain why the body can be modeled as an RC circuit.

4. Students will be able to explain how the body's fat free mass can be measured using bioelectrical impedance analysis.

5. Students will be able to explain how and why the body's impedance changes with frequency. 


\subsubsection{Computed Tomography}

This section describes a simple, safe apparatus that demonstrates the basic mechanism and concepts behind computed tomography (CT) scans without using potentially hazardous x-ray radiation. It is comprised of a photogate, rotary motion sensor, and other equipment commonly found in most physics teaching labs. Supplemental details about the activity can be found in reference [66].

\section{Back Projection}

Computed tomography creates images through a process called back projection. In this process, x-rays are attenuated by objects in the scanned area, which is recorded as forward projection data (Fig. 2.6). That data is spread onto a reconstruction of the scanned area by entering the projection data in every pixel of the reconstruction that lies along the original line between the source and the detector. This step is called back projection. After multiple back projections, an image of the original object(s) forms.

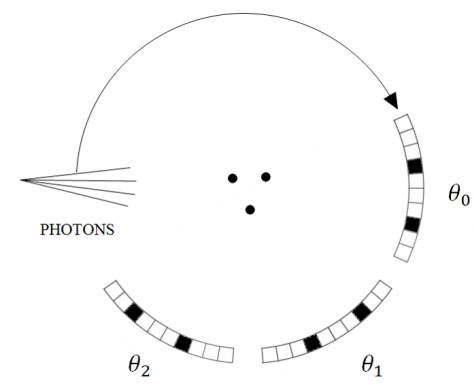

(a)

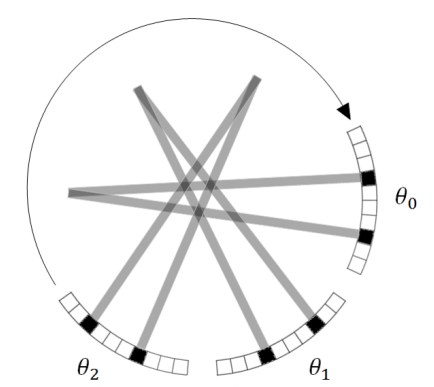

(b)

Figure 2.6: Basic reconstruction technique of CT. (a) A light source and a detector are rotated around an area. The black boxes in the detector arrays indicate where the light is being blocked. (b) The projection data are spread back onto a grid along the original line. The overlapping sections reveal the contents of the area. 


\section{CT Apparatus}

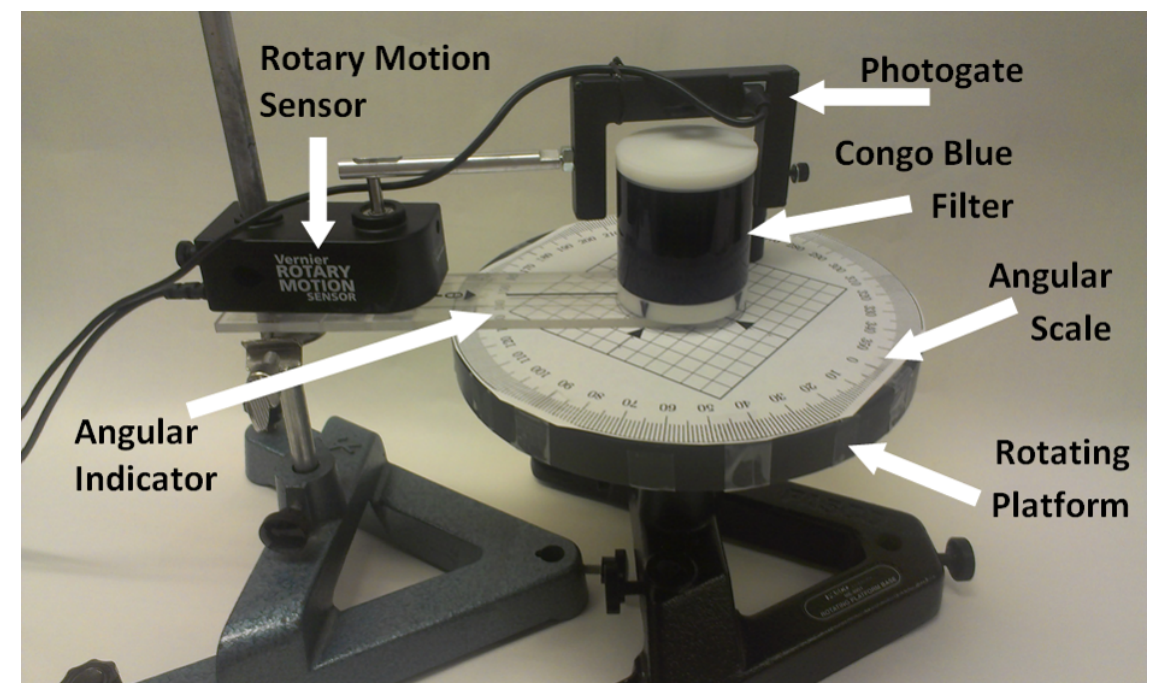

Figure 2.7: CT scanner with Vernier rotary motion sensor and photogate and an opaque enclosure made with a Congo Blue light filter.

The complexity and expense of acquiring a conventional x-ray-based CT scanner are generally too prohibitive for it to be included in undergraduate or high school physics laboratories. An inexpensive and safe alternative is a scanner based on an infrared light source. The most important components of our apparatus are a Vernier photogate using a light source with a peak wavelength of $880 \mathrm{~nm}$ [80] and a rotary motion sensor [81] (Fig. 2.7). To measure the rotation of the sample, we used a printable angular scale and an angular indicator made out of clear plastic. The angular indicator also maintains a constant distance between the rotational axis and the center of the enclosure. The sample is contained in an enclosure constructed from a clear plastic tube and the Congo Blue filter described in the radiography section [82]. The enclosure underscores the ability of CT to image obscured regions and is a good illustration for students of how different parts of the electromagnetic spectrum have very different electromagnetic characteristics. The enclosure was made small enough (7 cm diameter) so that it would fit inside the photogate. We placed a grid similar 
to Fig. 2.8(b) inside the enclosure so we could compare the original position to the resultant image.

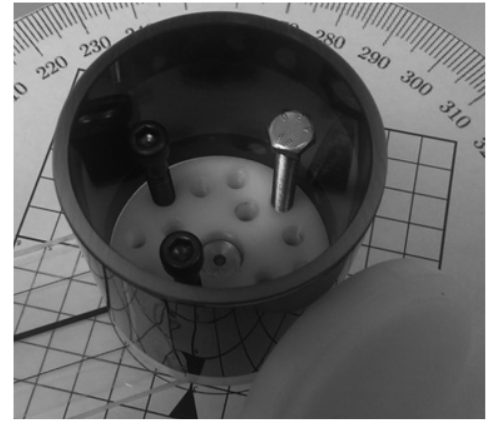

(a)

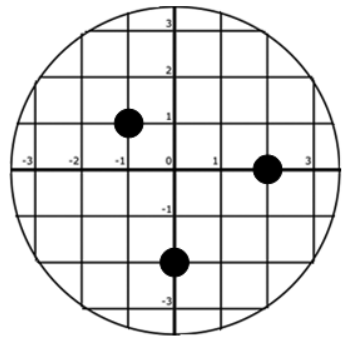

(b)

Figure 2.8: (a)The enclosure for the CT apparatus and the grid for securing the objects. (b)Positions of three cylinders inside the enclosure. The diameter of each cylinder is $0.6 \mathrm{~cm}$. The diameter of the enclosure is $7 \mathrm{~cm}$.

For simplicity, the sample is rotated about itself rather than the source/detector assembly around the sample, as in medical CT scanners. Unlike the continuous projection data (analog) collected in a real CT scanner, data collected by a photogate is digital (0-unblocked, 1-blocked). Although digital projection data does not completely reflect medical scanners, they do greatly simplify the apparatus design without losing key ideas behind CT.

\section{Operation}

The data from the photogate and rotary motion sensor were collected and graphically displayed by a program written with LabVIEW. The code is written for the Vernier sensors described above. Once the photogate becomes blocked the program calculates the equation of the line between the source and detector of the photogate relative to the grid in the enclosure. Using this equation, it then enters the projection data in every pixel of the reconstruction along that original line. The inputs of the program are (1) the distance between the rotational axis and the origin, also called the focus- 
center distance (FCD), (2) the angle between the FCD line and the negative x-axis $(\theta)$ and (3) the angle between the FCD line and the scanning line ( $\phi)$ (See Fig. 2.9).

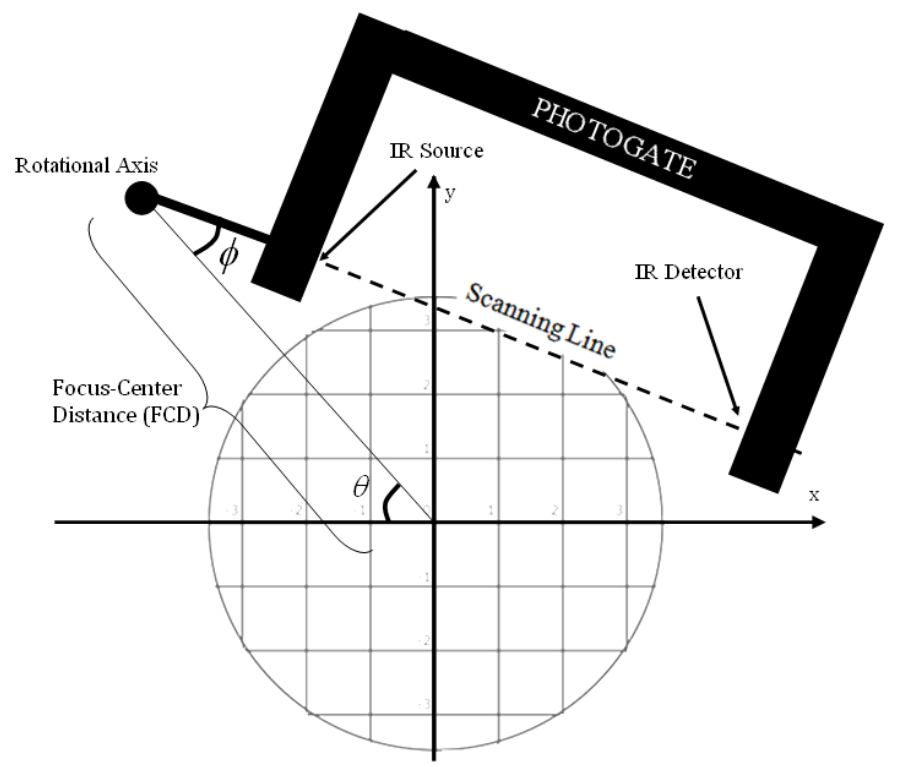

Figure 2.9: Geometry of the CT scanner.

The rotary motion sensor determines $\phi$, whereas $\theta$ is entered by the user and the FCD is a constant set by the plastic angular indicator (Fig. 2.7). Manual data entry is minimal, as the FCD needs to be entered only once and $\theta$ after every rotation of the rotational platform with the enclosure. The LabVIEW program collects and displays data in real time; thus, students are able to see the image formed step by step (Fig. $2.10(\mathrm{a}))$.

The images in Fig. 2.10 are of three identical cylinders, each with a diameter of $0.6 \mathrm{~cm}$, placed in the enclosure as shown in Fig. 2.8(b). The large ring around the cylinders in the images is the opaque enclosure which reflects the light from the photogate at shallow angles effectively blocking it. The time needed to complete a full scan is dependent on the number of passes (each having a unique $\theta$ ), the rotational speed of the photogate, and the resolution of the reconstruction, which can 
be increased or decreased to compensate for a computer's processor speed. Because more time is needed to enter data into the reconstruction as the resolution increases, computer calculations at high pixel counts can interrupt real time data collection. The images in Fig. 2.10 have $121 \times 121$ pixels, which is the highest resolution our system could manage in real time. The image in Fig. 2.10(b) is made with 36 passes $\left(10^{\circ}\right.$ increments over $\left.\theta=360^{\circ}\right)$, which is sufficient to create a quality image in a reasonable amount of time (about 20 minutes).

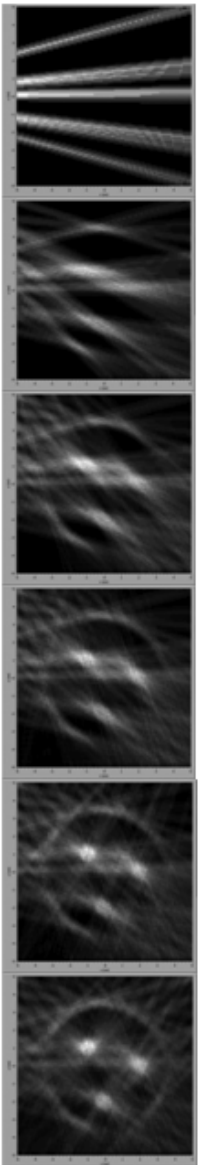

(a)

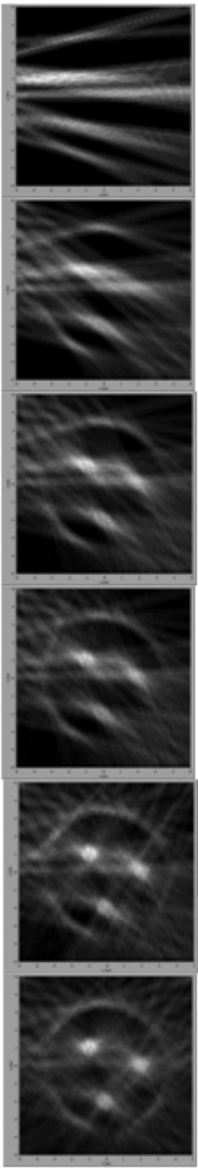

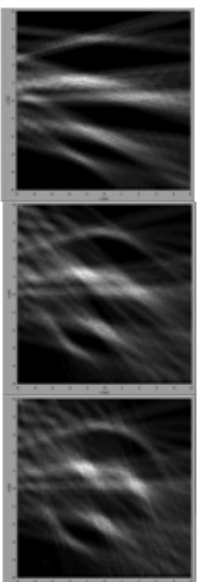
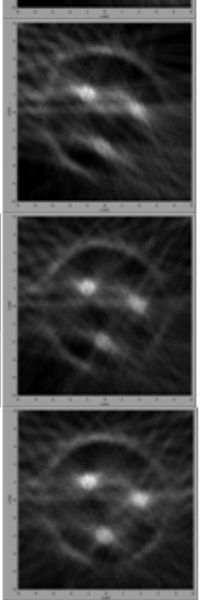

(c)

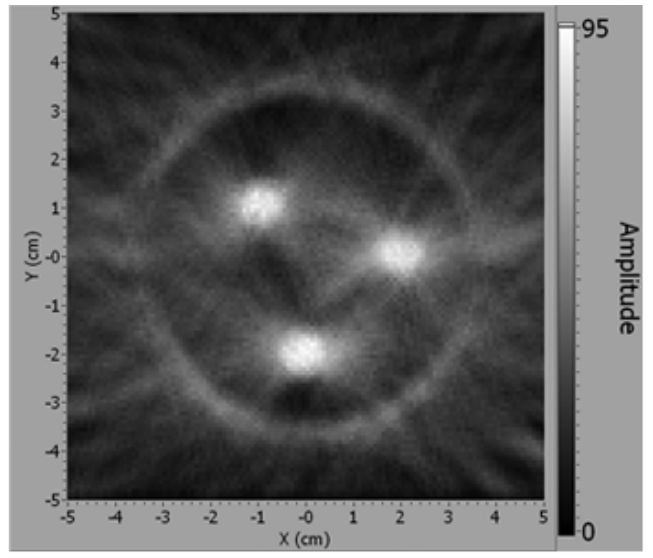

(b)

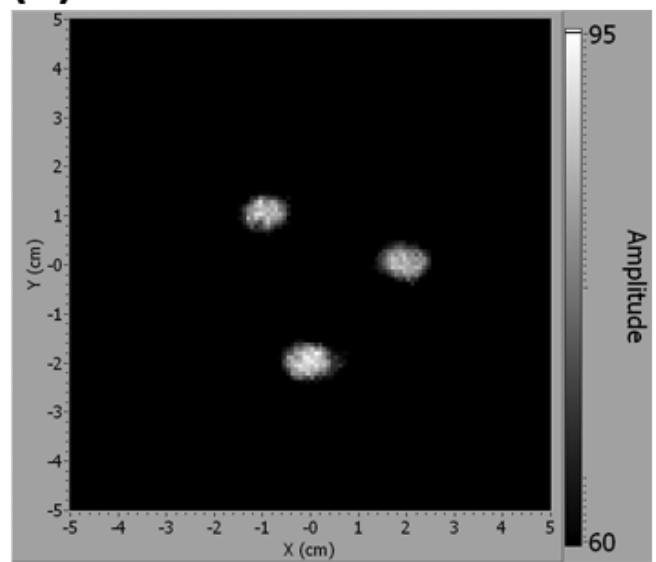

Figure 2.10: (a) Scans of the cylinders as shown in figure 2.8(b) taken in $10^{\circ}$ increments over $=150^{\circ}$. The ring around the cylinders is caused by the opaque enclosure, which reflects the light from the photogate at very shallow angles effectively blocking it. (b) A completed scan taken in $10^{\circ}$ increments over $=360^{\circ}$. (c) The same data from (b) after applying a window. Note that the lower boundary of the grey scale has increased which obscures pixels with lower values 


\section{Learning Goals}

Students who complete this lab should be able to describe multiple concepts related to attenuation and the electromagnetic spectrum. They should also have a good understanding of how a CT image is formed and what risks are involved. The specific learning goals are:

1. Describe the properties of substances that affect the penetration and characterize the rate of attenuation.

2. Compare the radiation exposure and its medical consequences of CT to normal planar x-rays and explain why this is so.

3. Represent the process of back projection with a schematic illustration and describe what the CT images represent.

4. Describe the advantage to images produced by back projection and contrast it with images produced in other ways.

5. Compare the pros and cons of short versus long scan times.

6. Compare and contrast the energy and atomic interactions of x-ray light to lower spectrum energy light 


\subsubsection{Optics of the Human Eye}

At HHU in Düsseldorf, five online labs were developed and have been an integral part of physics education for medical students since 2003. They can be used as a substitute for hands-on labs or as a supplemental tutorial to the physics lecture. The learning efficacy of the online labs and corresponding hands-on labs was compared in several studies, and no significant differences were found (e.g. [83,84]). Furthermore, the acceptance of the online labs at HHU had been evaluated with positive results throughout. [85] We transferred the activity on the optics of the human eye to PSU and studied the effectiveness of the transfer. Additional information on this study can be found in the published study [67].

\section{Design and Features of the Online Labs}

A physics lab course for medical students should primarily foster students' understanding of physics concepts that are relevant in medicine. Thus, the online labs that were developed at HHU cover physics content that is highly relevant for medical students. This content is embedded in the medical context throughout the online lab. Explanations, questions, and examples continuously stimulate the students to establish links between the physics content and the medical context. In several activities, the experimental setup itself hints at the medical context. For example, in the study presented here, students work with functional models of the human eye (see Fig. 2.11(a)). The model consists of a water basin (vitreous humor) with a curved glass (cornea) and a diffusing screen (retina). Behind the cornea, a flexible lens is inserted into the water basin. The lens is filled with oil and the focal length can be modified by pumping oil into or out of the lens (accommodation). Corrective lenses 
can be put in front of the "cornea" in order to compensate for myopia or hyperopia.

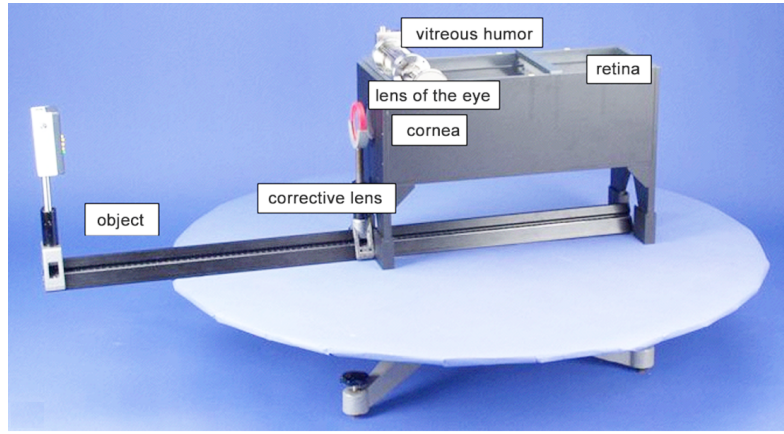

(a)

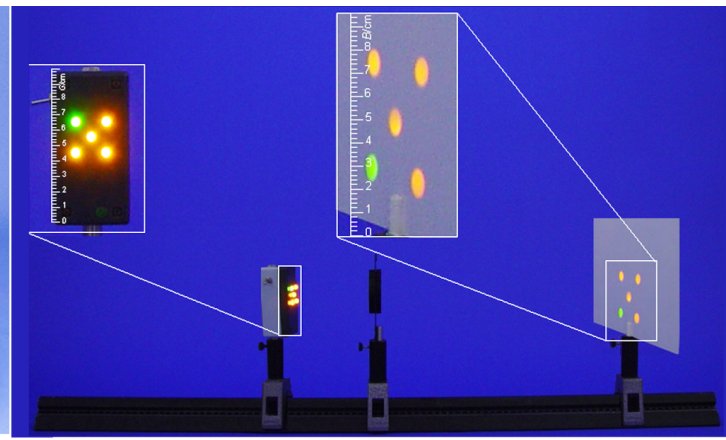

(b)

Figure 2.11: (a) Model of the human eye used in the online lab and (b) a screenshot from an ISE that allows for the investigation of imaging with a lens

The online lab consists of a hypertext with multimedia elements (e.g. [85]). It is accessible from any typical desktop or laptop computer with internet access. The hypertext offers a pre-structured learning path that allows students with little previous physics knowledge to work through the material. Multimedia elements, questions, and tasks require an interactive engagement with the content. In order to enlarge the authenticity of the observations and measurements, substantial multimedia elements of the online lab are digital representations of hands-on experiments. They are implemented in the form of interactive screen experiments (ISEs). [86] An ISE is a photo-realistic representation of an experimental setup that enables the students to manipulate several parameters directly within the representation of the experiment. They can grab and move objects with the mouse pointer and immediately observe the changes in the experimental setup and resulting measurements. Basic experimental setups to investigate refraction at surfaces or imaging with lenses as well as the functional model of the human eye (see Fig. 2.11(a)) are realized as ISEs and implemented in the online lab. Figure 2.11(b) shows a screenshot from an ISE that can be used to investigate the imaging with a lens. Students can move the screen (right) or the light source (left) and observe the image on the screen. An enlargement 
of the screen and the light source can be opened in order to measure the size of the object and the image.

Students have to perform and document measurements with the ISEs, draw conclusions from their measurements and observations, answer questions concerning the link between physics content and the medical context, and solve tasks that train students to use basic mathematical relationships. All entries are assigned to the registered user and stored on a server housed at the university. Each user retrieves his or her results whenever he or she logs into the online lab again, allowing students to take a break and continue where they left off at any point in the lab. Instructors or teaching assistants can review students' entries to all input boxes, comment on these entries, or demand revisions. Figure 2.12 shows a with the preview of an ISE and several input boxes for measurements and discussions.

Geometrical Optics $\Theta 3$ Image Formation in the Eye $\rightarrow 3.4$ Farsightedness

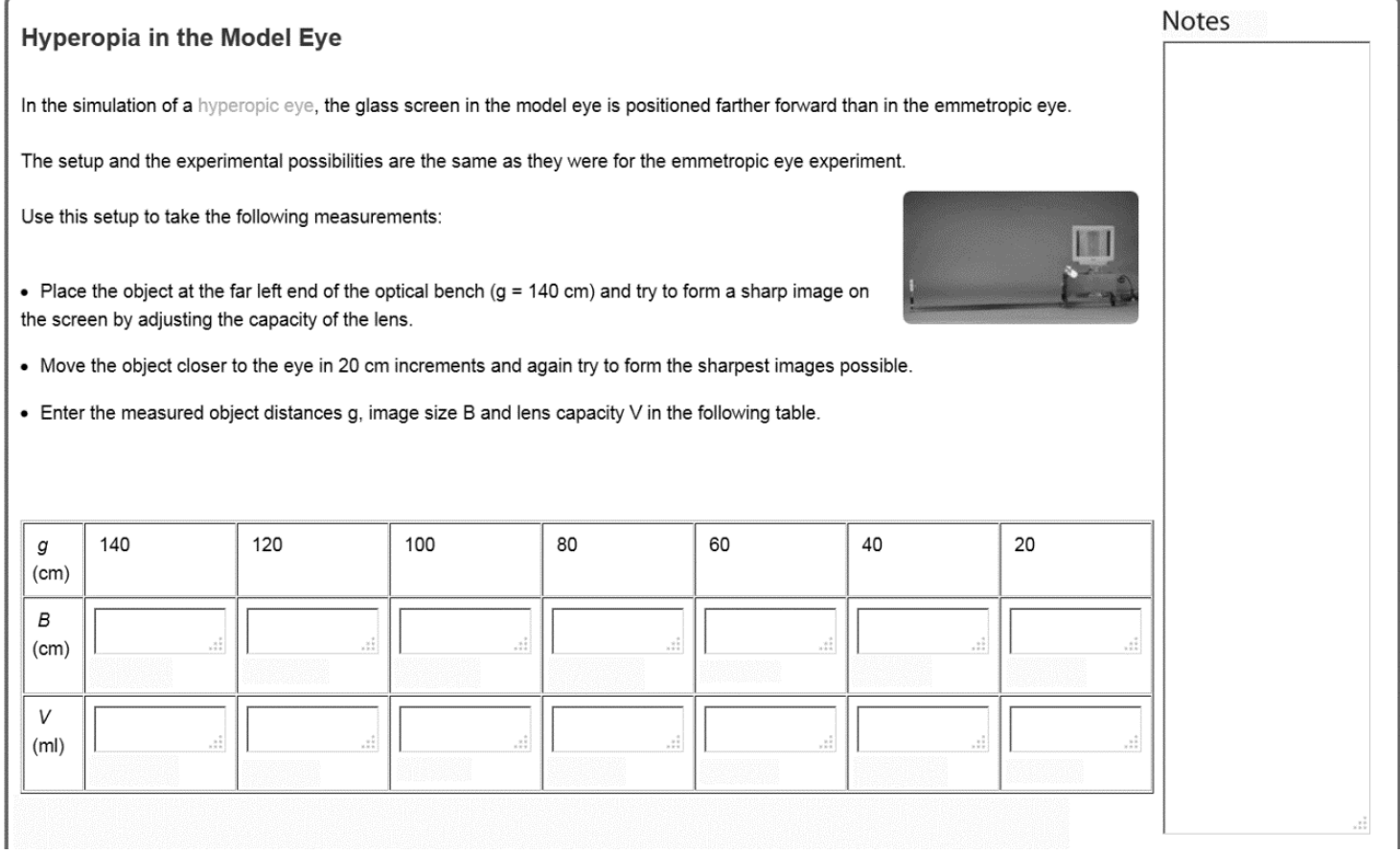

Figure 2.12: Screenshot of the online lab 


\section{Learning Goals}

The learning goals of this activity are primarily to describe concepts like refraction and geometrical optics as well as how they are important to human vision. After completing this lab students should be able to:

1. Describe reflection, refraction, and total internal reflection.

2. Describe differences between converging and diverging lenses regarding shape and image formation.

3. Use geometrical optics to find the location and size of an image formed by a lens.

4. Describe the anatomy of the eye (cornea, lens, vitreous and aqueous humors, and retina) including how each part plays a role in image formation.

5. Describe possible causes of nearsightedness, farsightedness, and presbyopia.

6. Explain how lenses correct these vision defects.

\section{Transfer of the Online Optics Module}

As part of the project involving the online modules exploring the optics of the human eye, we evaluated the effectiveness of the transfer. This required assessing the differences between the UDE and PSU cohorts in terms of (1) background, (2) learning gains, and (3) attitudes toward the modules. Only a brief summary of the findings is given here. More information can be found in the published study [67].

It was found that the PSU and UDE students in this study differ significantly in age, physics-related self-concept, and previous knowledge, but not in cognitive abilities. The differences between the cohorts was attributed to the different educational systems used in the USA and Germany.

The learning gain was significantly higher at PSU than at UDE. One possible 
reason for the difference in learning gain is that the UDE students demonstrated a higher previous knowledge than those from PSU. Therefore, the possible gain was more restricted than that for PSU students.

Students' attitudes toward the online lab were positive and quite similar at UDE and PSU. The assessment of "understandability" of the labs were also favorable for both groups. The physics-related self-concept proved to be an important factor influencing attitudes towards the online lab. 


\subsubsection{Radiography}

Electromagnetic radiation and the electromagnetic spectrum are an important part of the introductory physics curriculum. The discussion of light interaction with matter is of special relevance to pre-health and life science students as many diagnostic techniques and research tools are based on it. The specific aim of the apparatus and accompanying laboratory exercise described here is to demonstrate core physics principles underlying an essential medical imaging technique while promoting a conceptual understanding of properties of electromagnetic radiation, attenuation, and image formation through hands-on experimentation. Supplemental information on this activity can be found in reference [87].

\subsubsection{Apparatus}

Using x-rays in an educational setting can be expensive and require considerable safety precautions. However, the concepts underlying radiography can be taught using safe and inexpensive visible or near infrared (IR) light sources [26,66]. Similar to how invisible x-ray radiation is used to image the human body, we use invisible IR radiation to image the inside of an opaque enclosure. Both imaging methods result in a two-dimensional projection of a three-dimensional volume. The two-dimensional image gives a profile of the objects, which cannot be seen with the human eye, and the intensity of radiation present at the detector is used to characterize the composition of the concealed materials through a comparison of relative attenuation characteristics.

The principle components of the apparatus are an IR light source (Fig. 2.13(a)), an IR detector made of upconverting phosphor (Fig. 2.13(e)), an attenuation slide made of materials with differing opacities (Fig. 2.13(c)), and an enclosure, in which 

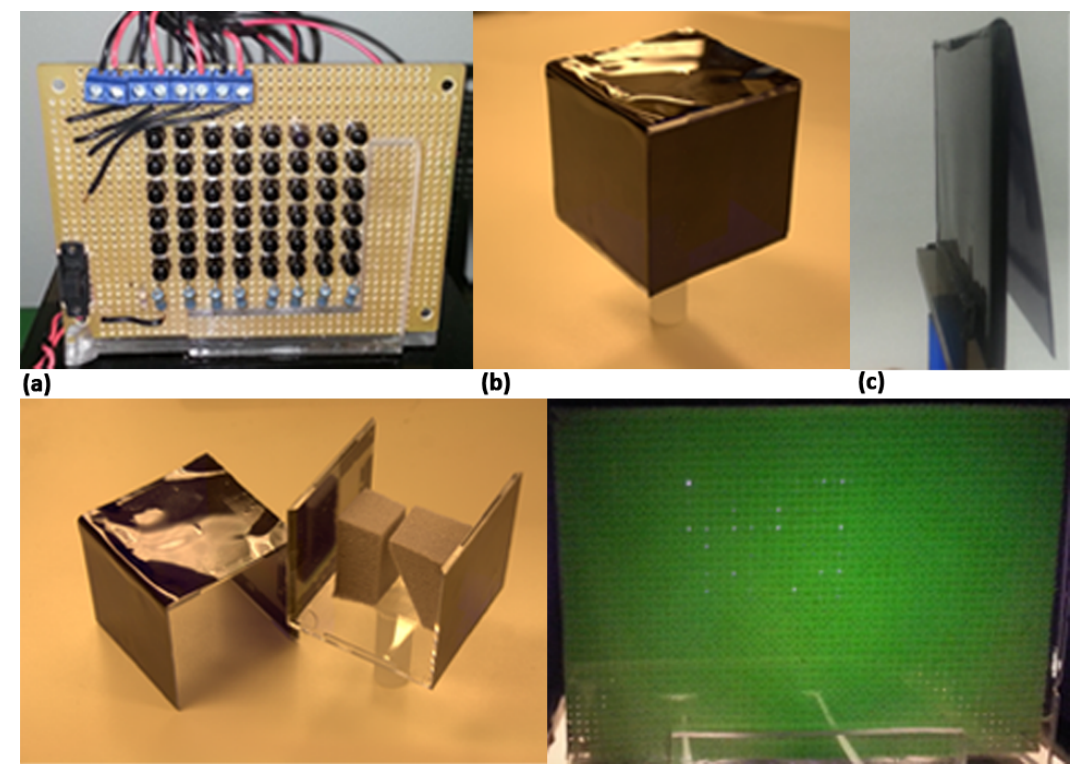

(d)

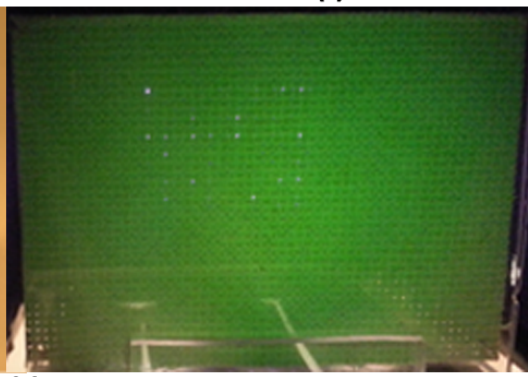

(e)

Figure 2.13: Radiography apparatus components: (a) infrared LED array, (b) enclosure mostly opaque to visible light but mostly transparent to IR, (c) slide with various attenuation coefficients, (d) objects concealed within the enclosure, and (e) detector screen emitting green light upon excitation by infrared light.

various objects can be placed (Fig. 2.13(b)and (d)). This enclosure is constructed with a Congo Blue filter, which is opaque in the visible spectrum, but transparent to IR light [82].

\subsubsection{Attenuation}

In the first activity, students explore attenuation with a slide constructed from the Congo Blue filter and two pieces of paper (construction paper and vellum) (Fig. 2.14). This slide enables the student to visually observe the difference in radiation transmission through each material by comparing differing intensities at the phosphor screen. These varying intensities can be used to infer the relative attenuation coefficients of the various materials in the slide.

Students are asked to observe the intensity of infrared light seen on the detectors 


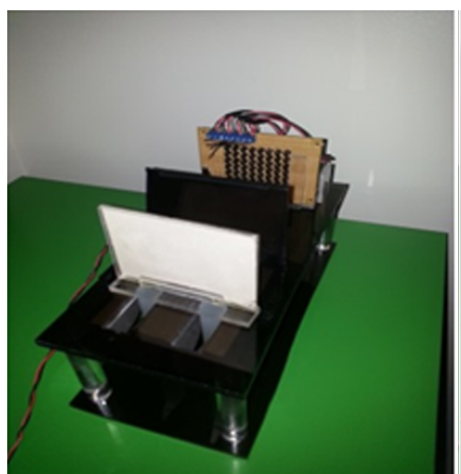

(a)

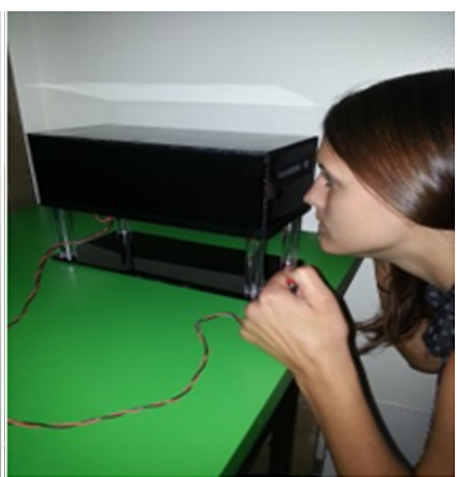

(b)

Figure 2.14: Arrangement for examination of attenuation effects on transmission. (a) Place the detector and slide on the base. (b) Place the lid on the base, hold the button, and view the image through the slot.

and compare them to each other as well as to analogous parts of the human body (Fig. 2.15). For example, the Congo Blue filter is largely transparent to IR light, similar to how skin tissue is largely transparent to x-rays. Conversely, the vellum used for the right half of the attenuation slide blocks a fraction of the light and resembles organs in radiological images. The construction paper used to cut out the shape of a bone in the attenuation slide blocks a majority of the emitted light, resulting in a dark area on the detector. This is analogous to bone and metal blocking x-ray transmission, resulting in unexposed areas on x-ray film.

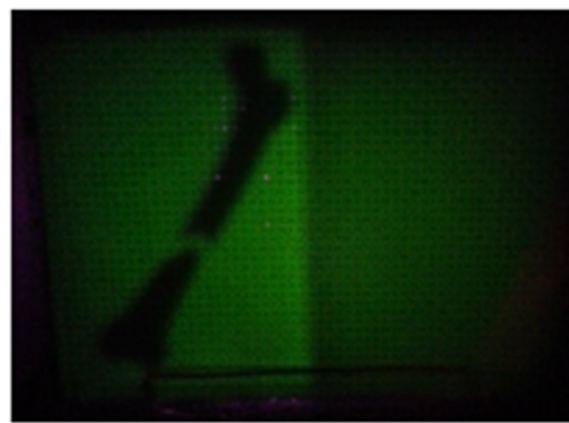

(a)

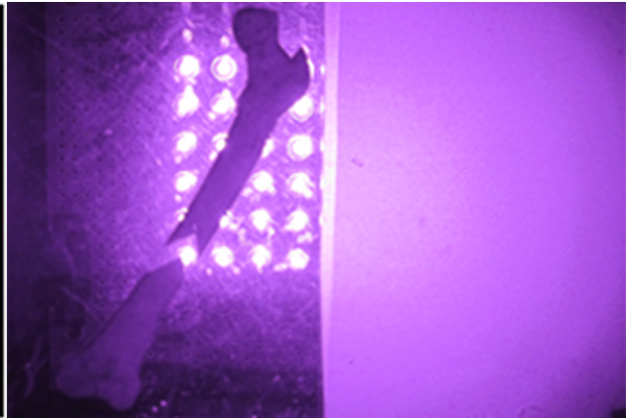

(b)

Figure 2.15: (a) Image of the analog up-converting phosphor detector and (b) digital image taken with a cellphone, displaying differences in image intensity corresponding to varying attenuation coefficients of materials in the slide. The left half (Congo Blue) of the slide is most intense, the right half (tissue paper) appears dim, and the bone (construction paper) appears dark as all transmission is blocked. 


\subsubsection{Planar Imaging}

In the second activity, students explore how three-dimensional objects appear in planar imaging. An enclosure surrounded by the Congo Blue filter causes the identity and position of any objects inside to be obscured from sight [Fig. 2.13(b)]. Students quickly discover that a two-dimensional image of a three-dimensional space is insufficient to determine the spatial arrangement of the unknown objects. For example, if two objects are in the enclosure, an image from only one angle will not provide a complete description of object orientation, number, and shape. By rotating the enclosure, students can view the contents of the box from multiple angles and thereby discern the shape and orientation of the objects contained within (Fig. 2.16). In this way, the activity can serve as an introduction to CT.

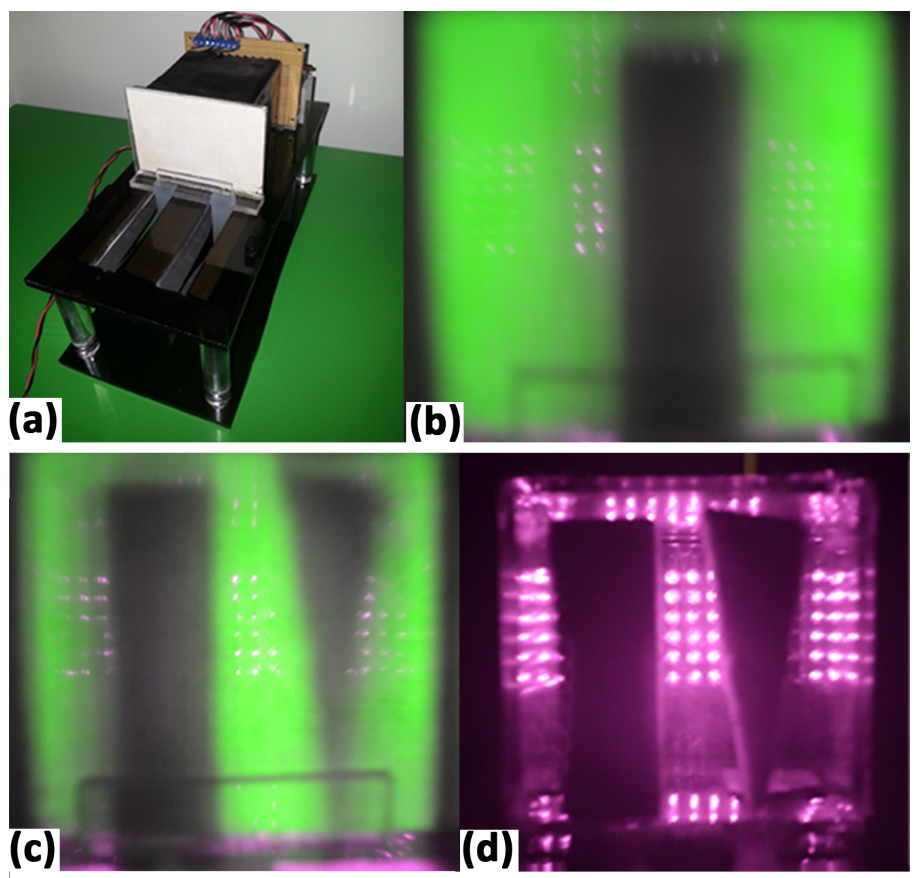

Figure 2.16: Planar images of concealed objects similar to Fig. 1(d). (a) Experimental setup for the planar imaging activity. Figures (b) and (c) show how additional information about the objects can be attained by viewing from two angles. Figures (c) and (d) illustrate the differences between analog (phosphor screen) and digital (cellphone camera) detectors. 


\subsubsection{Learning Goals}

After completing this laboratory, students should be able to describe a number of concepts related to the electromagnetic spectrum, attenuation, and x-ray imaging. The specific learning goals for this laboratory are:

1. Identify different regimes of the electromagnetic spectrum including x-rays, infrared, and visible light.

2. Describe the relationship between the wavelength, frequency, and energy of light.

3. Describe the properties of substances that affect the penetration and characterize the rate of attenuation.

4. Describe the way radiographic images are formed

5. Compare and contrast the usefulness and safety implications of planar imaging and CT scans. 


\subsubsection{Pulse Oximetry}

In this lab, students explored the physical concepts behind the pulse oximeter, a device commonly used in the medical field to measure a patient's pulse and the oxygen saturation of their blood. This is possible because of the different light-absorption characteristics of oxygenated and deoxygenated hemoglobin. By measuring the absorption of light by the blood at two different wavelengths and by comparing those measurements to empirical data, the relative concentration of oxygenated blood can be calculated. Additional information on this activity can be found in reference [68].

\section{Absorption and the Pulse Oximeter}

In the first part of the lab, students use a spectrometer to explore the absorption characteristics of different solutions of salt water. In this way, they are introduced to the different factors of the Beer-Lambert law of absorption such as the absorption coefficient. Next, students experiment with an actual pulse oximeter to explore how activities like exercise can alter the relative amount of oxygenated blood.

\section{Educational Pulse Oximeter}

In the rest of the activity students use an original device that simulates the function of pulse oximeters (Fig. 2.17(a)). The device has a LED that can emit either green $(700 \mathrm{~nm})$ or red $(565 \mathrm{~nm})$ light through a solution. The light sensor on the other side of the solution measures the intensity of the transmitted light.

A substance called bromothymol blue was used in the lab rather than actual blood. This substance is green in its neutral state and yellow in its acidic state (Fig. 2.17(b)), which can be achieved by exhaling onto the solution. The neutral and acidic states 


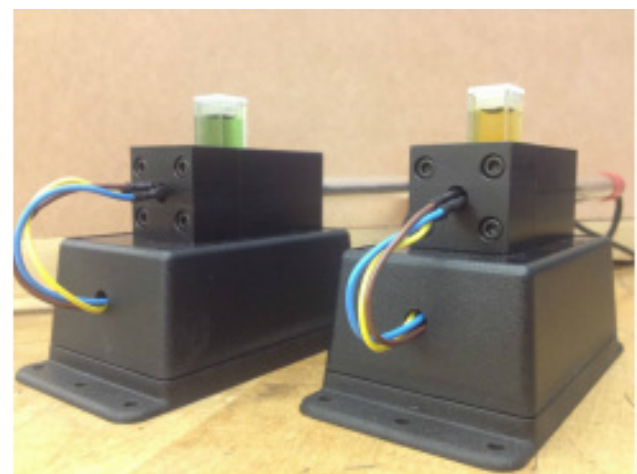

(a)

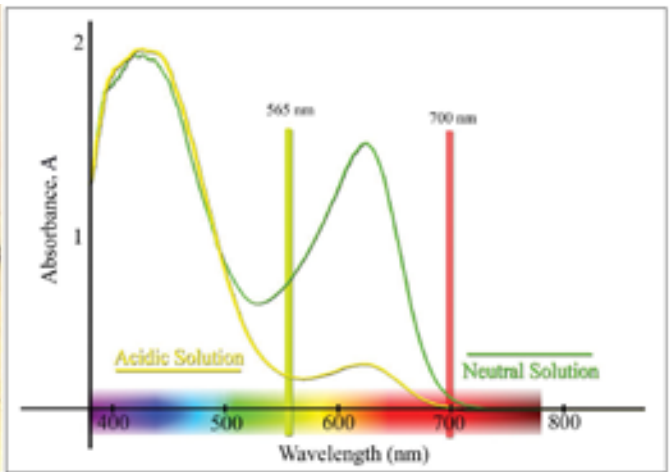

(b)

Figure 2.17: (a) Apparatus used in the pulse oximetry lab activity. (b) Absorption spectra of the bromothymol blue solution as measured by a spectrometer. (Source Ref. [68])

are analogs for oxygenated and deoxygenated blood. As an option, students can calculate the $\mathrm{pH}$ of the bromothymol blue solution using the data acquired with the simulated pulse oximeter in a manner similar to calculations used by medical pulse oximeters to find blood oxygen saturation.

\section{Learning Goals}

The learning goals of this lab include how pulse oximeters utilize the concept of absorption. After completing this lab, students will be able to:

1. Describe that the pulse oximeter device can measure both blood oxygen levels and pulse.

2. Correctly describe the correlation between oxygen content and blood color.

3. Explain what spectral information is collected and how an inference of blood oxygen content is made from this.

4. Explain why both red and infrared light is used by pulse oximeters.

5. Create a map of the $\mathrm{pH}$ conditions and the use of red/green light in the bromothymol blue experiment to the color change and oxygenation of blood.

6. Describe why the pulse oximeter measures the ratio of oxygenated hemoglobin to non-oxygenated rather than total oxygen content. 


\subsubsection{Electrocardiogram}

This activity explores the fundamental electrical components and characteristics of the electrocardiogram (EKG or ECG), which is a commonly used medical device that monitors the activity of a patient's heart. The primary functions of the EKG are to amplify the electrical signal created by the heart and to filter out signals from additional sources (Fig. 2.18). This laboratory activity explores each of these functions. Additional information on this activity can be found in reference [69].

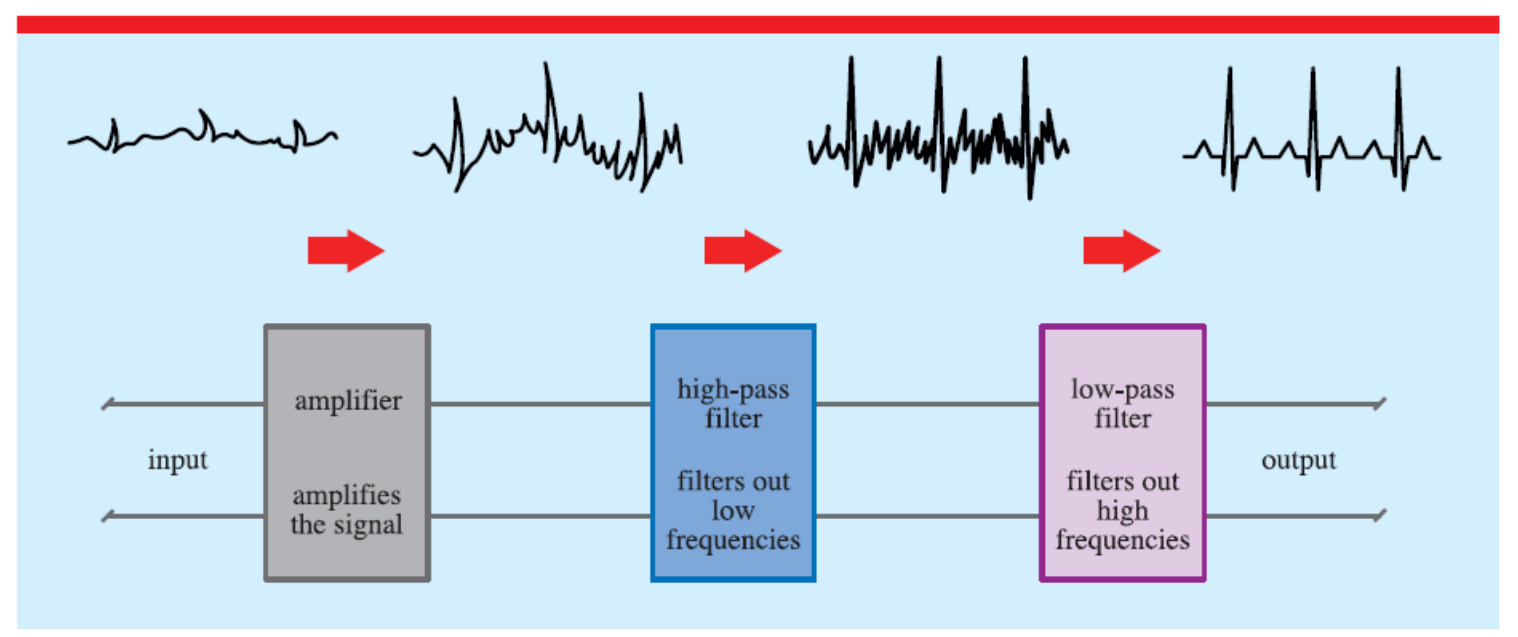

Figure 2.18: Fundamental functions of an EKG sensor. (Source Ref. [69])

\section{AC Circuits and Filters}

EKG sensors are able to detect electrical signals from other sources than the heart. One of the functions of the EKG is to filter out these additional signals through electronic filters. In the first section of the lab, students combine discreet electrical components like resistors, capacitors, and inductors to build similar filters. Specifically, students explore how high and low pass filters can be designed to reject AC voltages oscillating beyond a predetermined cutoff frequency. The activity also demonstrates 
to students how to combine these filters to create a band-pass filter similar to the kind used in EKG sensors.

\section{The EKG Sensor}

The rest of the lab focuses on the function of an EKG sensor itself. This begins with EKG measurements of student volunteers to determine the frequency of the pulses created by the heart as well as the causes of any additional electrical signals that will need to be filtered out.

Next, students experiment with the amplification characteristics of the EKG by using a signal generator to create a small potential across a voltage divider. The EKG and a standard voltage probe are both used to measure the potential and students compare this data to calculate the gain of the EKG.

In the final section of the lab, students use two signal generators to create a single AC potential composed of two superimposed waveforms. Students experiment with different signal frequencies to determine the range of frequencies that the band-pass filter of the EKG sensor attenuates. They find that the sensor is of course designed to be most sensitive to the frequencies relevant to the signals created by the heart and to attenuate frequencies from other sources. 


\section{Learning Goals}

After completing this lab, students should be able to accurately describe the function of the major electrical components of the filter used by an EKG.

1. An EKG sensor measures and amplifies output signals from the heart in the magnitude of millivolts in the approximate frequency range of $1 \mathrm{~Hz}$ to $20 \mathrm{~Hz}$.

2. An EKG sensor functions as a band-pass filter to filter out environmental noise and to attenuate frequencies above and below the range of the human heart.

3. An AC circuit with a function generator, inductor, and capacitor can act as a band-pass filter.

4. In an $\mathrm{AC}$ circuit, the inductor functions as a low-pass filter that allows low frequency signals to pass through and attenuates high-frequency signals.

5. The capacitor functions as a high-pass filter that allows high-frequency signals to pass through and attenuates low-frequency signals.

6. In an $\mathrm{AC}$ circuit, the equation $f=1 /(2 \pi \sqrt{L C})$ can be used to calculate the value of the resonant frequency.

Extensive analysis of these learning goals has been carried out. The results are available on the project website [88]. I was not involved in this analysis; therefore the results will not be discussed here. 


\subsubsection{Kinesthetic Activities}

Educators have found that kinesthetic involvement in an experiment or demonstration can engage students in a powerful way [89-91]. With that as our goal, we developed three activities that allow students to connect with and quantitatively explore key physics principles from mechanics with three fun physical challenges. Each activity uses an original, real-time data collecting program that offers students and educators a simple, clear method to demonstrate various physics concepts including: (1) impulse, (2) momentum, (3) center of mass (COM), and (4) kinematics.

These activities are designed to be a contest between students, who are challenged to use what they have learned about the relevant physical principles to improve their score. The activities have been used in outreach events and the mechanics section of the algebra based introductory physics course at PSU. Additional information on these activities can be found in reference [70].

\section{Impulse-Momentum Theorem}

The impulse-momentum theorem states $F \Delta t=m \Delta v$, where $F$ is force, $t$ is time, $m$ is mass, and $v$ is velocity. To explore this relationship, participants are asked to jump from a small platform onto a force plate. The program plots the measured force from the contestant's landing in real time (Fig. 2.19). The vertical axis of the plot is the ratio of the measured force, or apparent weight, to the weight of the contestant, which is measured before the jump. In this way contestants' weights remain private and people of different weights have a chance to compete on equal footing. The score represents the peak force of the landing as a multiple of the participant's weight. The contestants are challenged to use the concepts learned from the impulse-momentum 


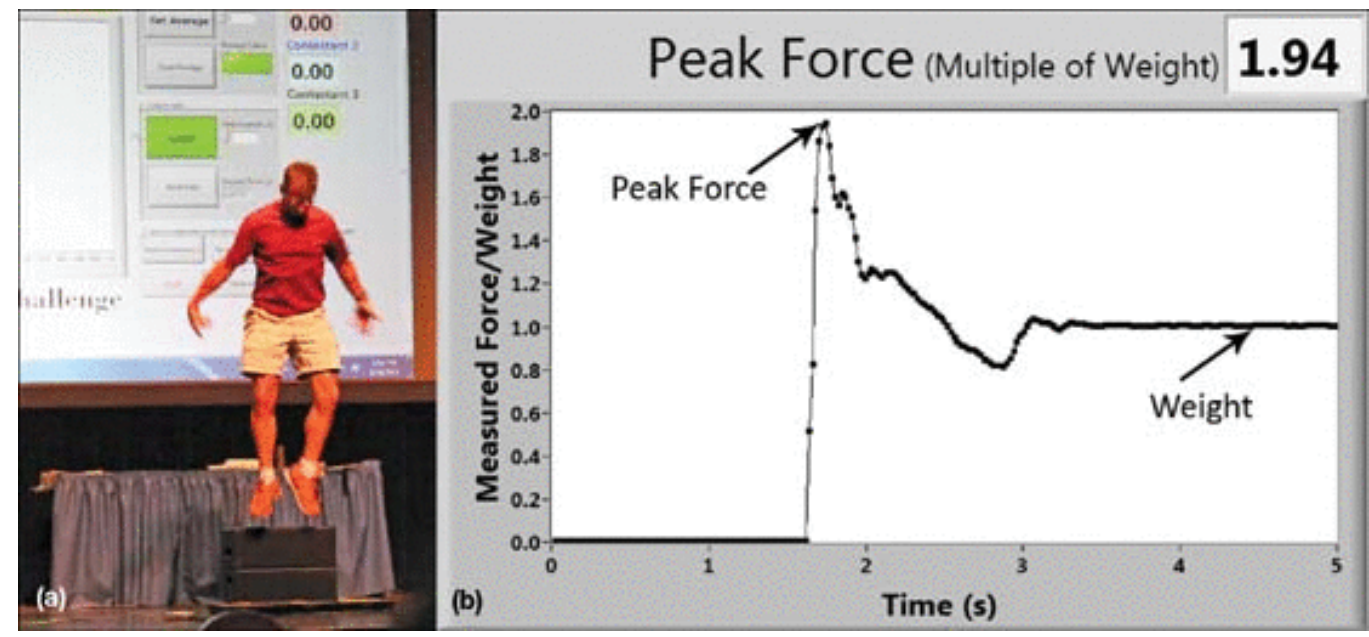

Figure 2.19: (a) A volunteer jumps from a small platform onto a force plate. (b) Force plate data of a volunteer jumping off a chair. The volunteer's weight is measured before the jump but not displayed (photo courtesy American Association of Physics Teachers).

theorem to get the lowest score.

Because the contestant ends the collision with the floor at zero velocity, the peak force of the impulse is dependent on the duration of the landing and the contestant's velocity immediately before landing, which itself is a function of the contestant's starting height. In the classroom, students are able to verify these relationships by experimenting with the dependent variables: starting height and landing time. Students can also discuss how a person's height may impact the chances for a low score. For example, students can lower their scores by crouching in the chair before jumping, which lowers their COM toward the floor and decreases their final velocity. They can also lower their scores by increasing the duration of the landing by bending their legs during impact.

\section{Balance Challenge}

For an object to remain upright, its COM must be above its base of support. If the base of support is small, staying balanced can be difficult. For example, it is more 
difficult for a person to balance on one foot than on two, and even more difficult to balance on their toes. By using a force plate, we can measure the vertical component of the stabilizing forces a person exerts while balancing. Like in the previous activity, the ratio of the measured force divided by the person's weight is displayed. This ratio fluctuates as the person tries to maintain balance and the standard deviation is used as a figure of merit. The smaller the standard deviation of the force plate data, the less a person is adjusting and therefore the better the balance.

In this demonstration, three contestants are each asked to balance on a force plate on one leg and with closed eyes. The program collects and displays the data from all three force plates in real time. While the contestants are balancing, their scores are continually being updated according to the formula

$$
\text { Score }=100 \% \times(\text { weight }-\sigma) / \text { weight },
$$

where $\sigma$ is the standard deviation of the measured force plate data. The standard deviations are changing as the measurements are updated, which means the scores change in real time during the 20 second balance challenge.

As a classroom activity, students can experiment with different ways of balancing and immediately visualize the effect upon their scores. For example, one student could stand on two feet while another stands on one foot. Students can also explore the large role that sight plays in helping them balance. Contestants 2 and 3 shown in Fig. 2.20 were both standing on one foot, but Contestant 3 has a lower score, largely due to his eyes being closed. Contestants may also explore how holding a long pole or a rotating wheel as a gyroscope may help them in maintaining their balance. Besides the discussion of the COM, this contest is a useful way to introduce students to the idea of the standard deviation. 


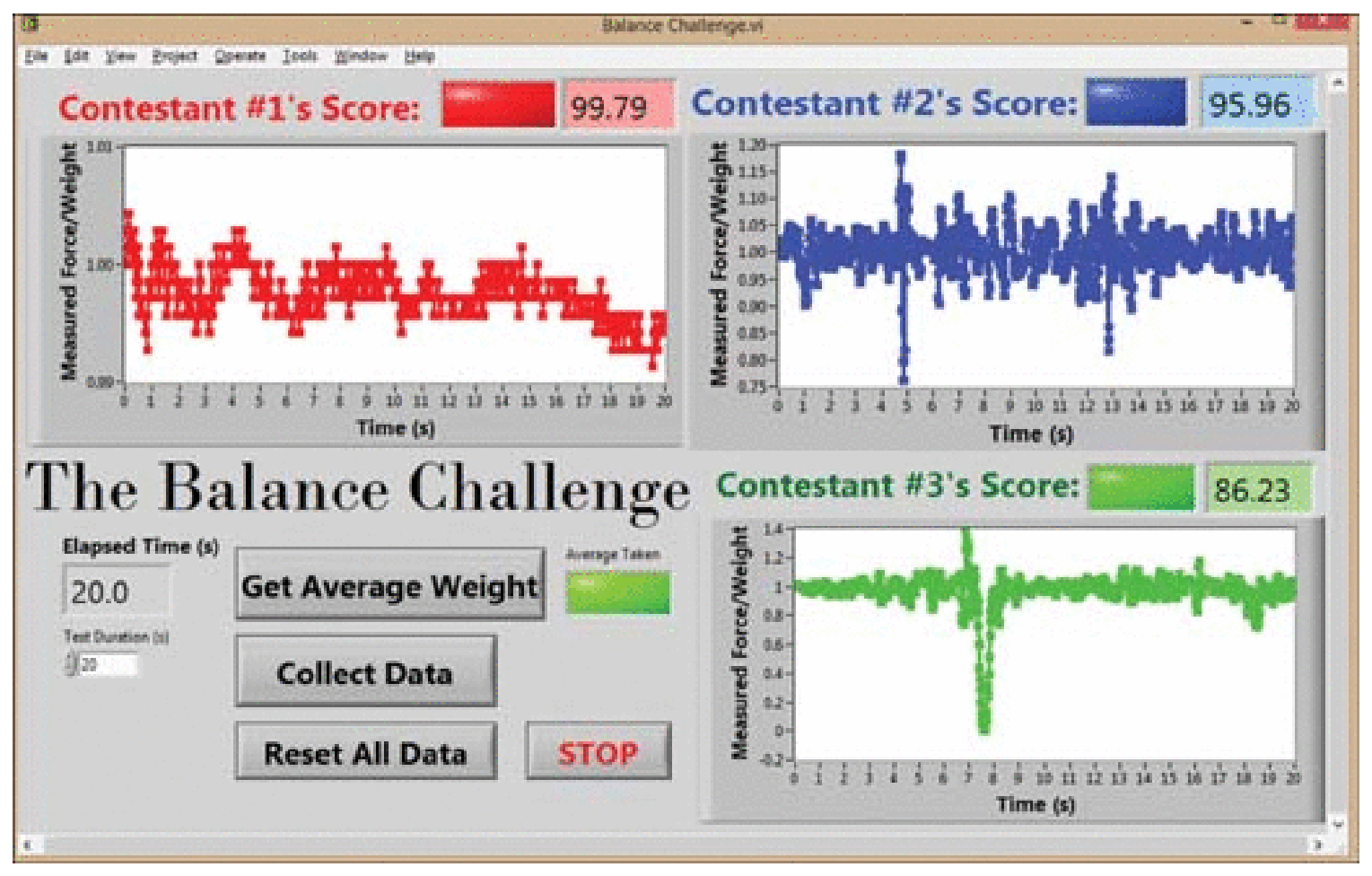

Figure 2.20: Screen capture of the program used in the balance challenge. The graphs display the force plate data of three volunteers. Contestant 1 is balancing on two feet. Contestant 2 is balancing on one foot with open eyes. Contestant 3 is balancing on one foot with closed eyes. Note that at approximately 7.5 seconds Contestant 3 lost his balance and stepped off the force plate.

\section{Kinematics}

Once an object leaves the ground, the motion of its COM will follow a parabolic trajectory. This is even true of an object that is able to change its mass distribution mid-flight, although it may be difficult for an observer to follow the motion of the COM. One such example is when a person moves his arms and legs while jumping, resulting in sections of the body following non-parabolic trajectories.

Our third program is able to track the position of an object in sequential images captured from a webcam and plot its trajectory in real time. We wanted to track the motion of the contestant's head during a jump, so we tasked the program to follow an orange ball attached to a bicycle helmet (Fig. 2.21(c)); this was both an easy target 

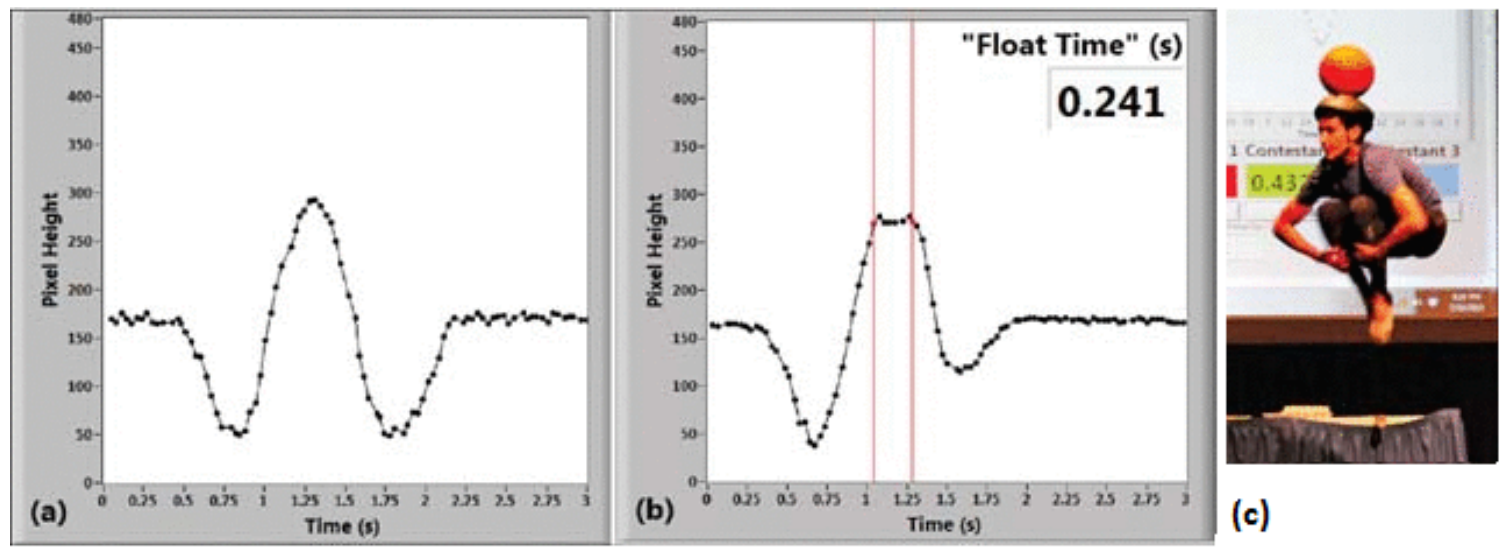

Figure 2.21: Trajectory of a ball attached to a helmet worn by the contestant for (a) a normal jump and (b) a jump in which the contestant tucks in his arms and legs. (c) By changing the position of his center of mass, the contestant's head follows a nonparabolic path.

for the program to track and an eye-catching prop for the stage. The program can easily be changed by the user to follow other indicators, including a spot of colored paper or garment.

For this activity, the contestant's task is to jump while maximizing the duration of the floating effect. The contestants are asked to jump straight up and move their bodies in such a way as to cause the trajectory of their heads to deviate from a parabolic function. One way this can be achieved is by closely tucking one's arms and legs in toward the body while in the air. Figure 2.21 shows the plots of two jumps: one straight up and down as well as one in which the contestant jumps and tucks in his appendages, as in Fig. 2.21(c). The graphs in Fig. 2.21 plot the vertical coordinate of the pixel containing the center of the orange ball. As can be seen in the first plot, the contestant's head is described by a parabolic function while jumping straight up and down. However, in the second plot the contestant is able to achieve the "floating" illusion by tucking in his arms and legs while jumping. The "float" time can be measured using the cursors on the graph. 


\section{Learning Goals}

These activities focus on concepts important to biomechanics including the impulse momentum theorem and the center of mass. They are therefore components that could be readily adopted for course reforms planned for fall term in the 2017-2018 school year. The specific learning goals for these activities are:

1. Apply the impulse momentum theorem.

2. Describe the concept of center of mass and explain how it can affect a person's ability to balance.

3. The center of mass of a projectile will always move in a parabolic trajectory, while individual parts of the projectile may not.

As of yet, these activities have been used as in-class demonstrations with student volunteers. No formal assessment of the learning goal has been conducted at this time. In addition to the fall term introductory physics course, these activities were also presented as part of the "Physics of Ballet", a performance done with our partners from the Oregon Ballet Theater. This show was performed at the Oregon Museum

of Science and Industry's Science Pub series [92] and at the demo show of the 2013 American Association of Physics Teachers summer conference [93]. 


\subsection{Multimedia Content}

This project aims to merge strong educational content with the benefits of visual media. Videos add a visual component to text-based learning content. However, in order to utilize this media for educational purposes it must be integrated with appropriate educational content. The material described in this chapter is designed such that it enables a physics instructor with a traditional physics background and without formal training in the biomedical field to teach a biomedically-focused physics course. More information about this material can be found at the project webpage. $[94]$

\subsubsection{Expert Interviews}

In 2012, we began to videotape guest lectures presented by our collaborators in the Physics in Biomedicine course at PSU. We decided that the format, setting, audio, and video quality of these recordings would not be engaging for students to watch at home. However, we used the recordings to determine which collaborators would be most suitable for videotaped interviews.

Generally, we conducted these interviews at the expert's office, hospital, or laboratory, as this provided a professional and relevant background. The interview format allows us to get both well-executed and concise video clips. Six to ten clips with a total length of about 30 minutes comprise one module. The average length of a video is about 4 min, and clips vary in length from 2 to 8 minutes. To keep students engaged, we overlay the interview footage with figures, images, animations, and video footage of actual equipment or procedures. We found that existing online material was insufficient for our needs and frequently copyright protected, so we opted to produce 
most of this footage ourselves. The completed videos are available on the YouTube account for this project [46].

Figure 2.22 shows images from the interview recorded at the Clinical and Translational Research Center at Oregon Health and Science University and images from the LASIK video module recorded at Teplick Custom Vision.

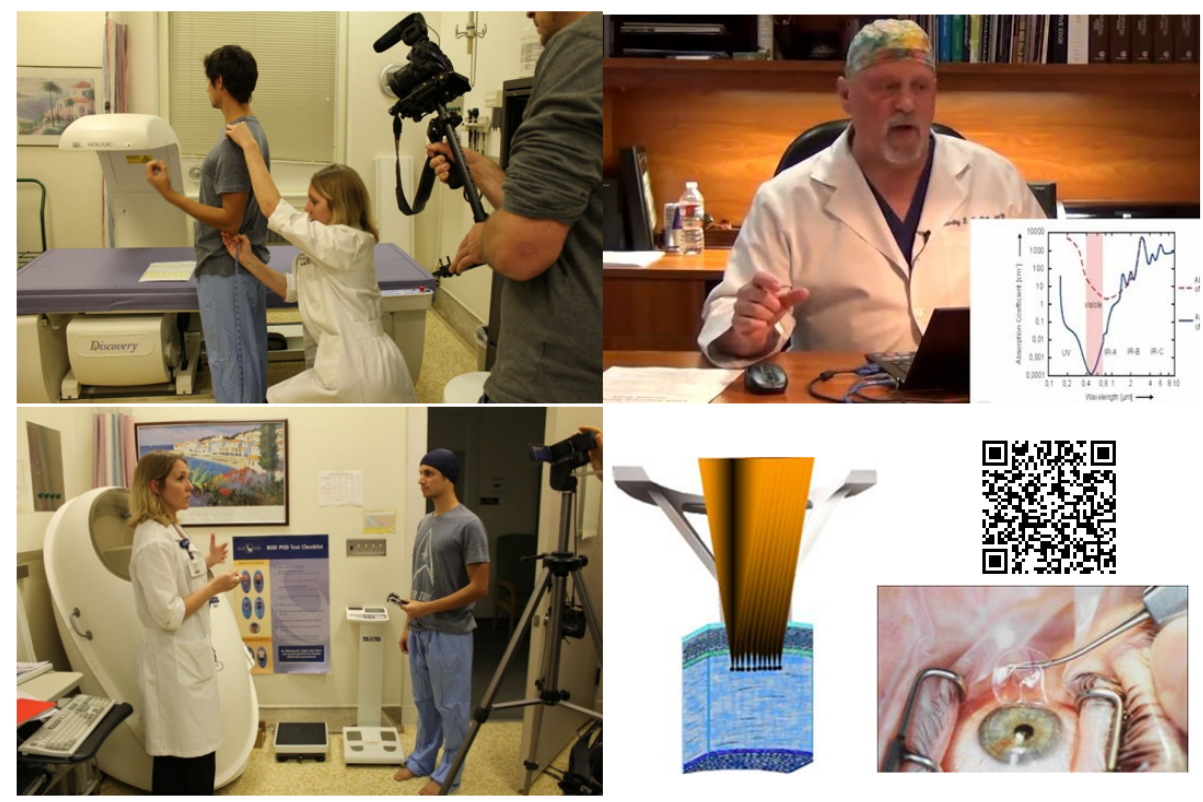

Figure 2.22: Left panels An undergraduate pre-health student volunteer has his body composition measured and prepares to step into the Pod Bod, a device that measures body composition by air displacement. Right panels Screenshot of a video clip with a LASIK surgeon explaining the different types of lasers he utilizes during surgery. Schematic showing the cutting of the corneal flap with focused laser pulses. Image of the eye with open flap before reshaping of the cornea (courtesy Teplick Custom Vision). QR code of a video clip on LASIK.

Table 2.2 is a summary of the biomedical expert interviews. Ultrasound, endoscopy, microscopy, LASIK, and pulse oximetry are all modules used in the introductory physics course. The modules on computed tomography, body composition, radiation therapy, PET, and MRI are currently used in the intermediate level Physics of Biomedicine course. The physical therapy and cardiovascular modules are the most recent and will be used in the 2017-2018 school year at PSU. 
Table 2.2: Summary of interviews with biomedical professionals.

\begin{tabular}{ll}
\hline \hline Medical Topic & Expert(s) \\
\hline Ultrasound & Radiologist \\
Endoscopy & Laparoscopic surgeon \\
Microscopy & Microscopist \\
LASIK & LASIK surgeon \\
Pulse Oximetry & Laparoscopic surgeon, Research fellow \\
Computed Tomography & Radiologist \\
Body Composition & Bionutritionist \\
MRI & Radiologist \\
Radiation Therapy & Radiation oncologist, Medical physicist, Dosimetrist \\
PET & Professor of diagnostic radiology \\
Flow Cytometry & Pathologist, Clinical laboratory scientist \\
Physical Therapy & Professor of physical therapy \\
EEG & Neurologists \\
Neurophysiology & Neurobiology researcher \\
EKG & Cardiologist, Certified medical assistant \\
Cardiovascular System & Cardiologist \\
Biomechanics & Physical Therapist \\
\hline \hline
\end{tabular}

\subsubsection{Course Text}

The text is a technical discussion of the biomedical application and serves as a fundamental scientific introduction to the topic. Some of the text contains physics concepts that can be integrated into different modules. Table 2.3 summarizes the main biomedical applications covered by the course text. All the chapters are used in either the introductory course, the intermediate course, or both. A sample of the text is shown in Fig. 2.23.

Table 2.3: Summary of chapters in the course text.

\begin{tabular}{ll}
\hline \hline Topic & \\
\hline Ultrasound Imaging & Computed Tomography \\
Physics in Endoscopy & Body Composition Analysis \\
Microscopy Physics & Radiation Therapy \\
LASIK & PET \\
Pulse Oximetry & Physical Therapy \\
Fluids in the Cardiovascular System & MRI \\
\hline \hline
\end{tabular}


Video: LASIK Custom Wavefront Technology

\subsubsection{Wavefront Aberrations}

In geometric optics, incoming parallel rays of light that enter a converging lens meet at the focal point of the lens. Conversely, light rays that originate at the focal point of a lens will be parallel to each other after passing through the lens.
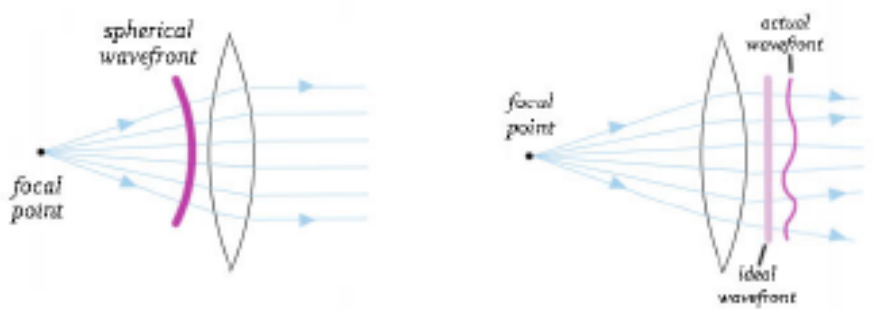

Figure 4.2: Wavefront for an ideal (left) and non-ideal (right) lens. Note that the light rays exiting the ideal lens are parallel, while those exiting the non-ideal lens are not.

A lens that has aberrations results in departures from the ideal wavefront in the light that passes through it.

In the Hartmann-Shack sensor, light leaving the eye passes through a lens array before being captured by a CCD (charge-coupled device, used in digital imaging) camera. Light from a perfect wavefront (left) and light from an aberrated wavefront (right) passes through a lens array and is received by a CCD camera:

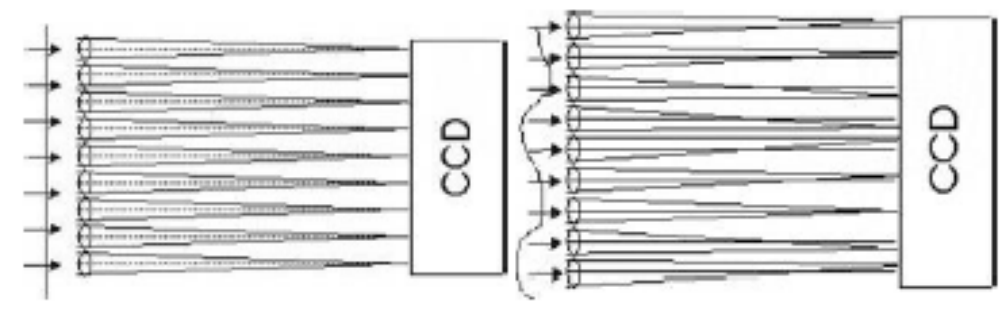

Figure 4.3: Lens array in the Hartmann-Shack sensor for an ideal (left) and non-ideal (right) wavefront

Figure 2.23: Sample course text from the chapter on LASIK

\subsubsection{FlipItPhysics}

For a delivery platform of the multimedia content, we employed the FlipItPhysics Framework (FIPF), which linked the expert videos from our YouTube channel. [46] The FIPF is the online platform used for the introductory physics text by Gladding 
et al. [63]. While we did not use any of the content from this course, we did use the same structure of pre-lectures, checkpoint questions, and homework. The FIPF allowed for seamless integration of multimedia content and detailed assessment of student responses that can be utilized with our medical physics content at no cost to PSU or students.

\subsubsection{Pre-Lecture Questions}

The pre-lecture questions are intended to ensure that students watch the videos and assimilate their content. Students receive immediate feedback if their answer is correct and have multiple attempts to obtain the correct answer. The questions are frequently designed so that multiple answers on a given multiple-choice question are correct and aim to discourage guessing as a successful strategy. The questions are written such that students can obtain the correct answer if they engage carefully with the video content. Question (1) is an example from the LASIK module (Fig. 2.24). The correct answers are in italicized bold font. The histogram shows the number of trials students needed to obtain the correct answer. A more detailed analysis of the students' log files revealed that students most commonly missed answer E.

(1) What are some examples of different types of lasers interacting with different types of tissues/materials that Dr. Teplick mentions?

A. IR-B lasers that affect skin tissue

B. blue-green lasers that affect hemoglobin

C. IR-A lasers that affect gum tissue

D. UV lasers that affect corneal tissue

E. lasers that affect melanin

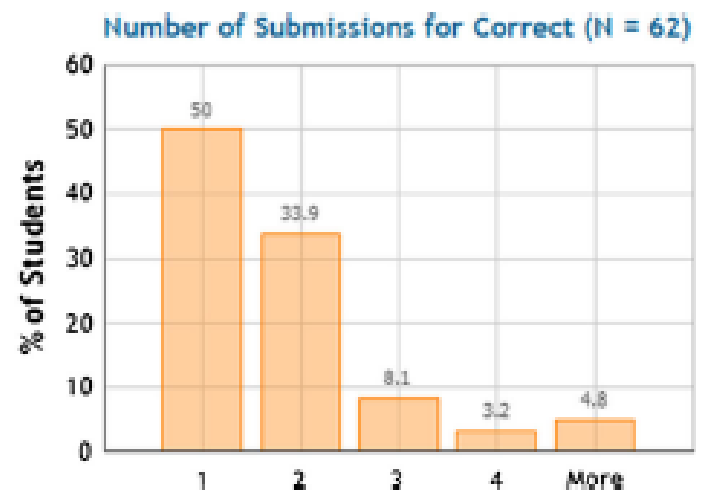

Figure 2.24: Sample pre-lecture question and number of trials students needed to get the correct answer 


\subsubsection{Checkpoint Questions}

Checkpoint questions are focused on core physics concepts, often probed in a biomedical context. The questions are meant to challenge students, provide inspiration for in-class discussion, and assist the instructor to target in-class instruction on material that requires additional focus. Many questions are presented in an open-ended essay format. This can be beneficial to the instructor because student answers are not guided and therefore are sometimes more revealing of student thinking. For example, a question used in the microscopy module would be:

"There are two different equations for the angular magnification of a magnifying glass: $M_{\alpha}=N / f$ and $M_{\alpha}=N / f+1$. Why is this the case and when do you use which equation?"

Multiple-choice questions are also commonly used in the checkpoints. Feedback to students on these questions is delayed until after the class meeting to encourage student discussion before or during class. Students receive participation credit for a good faith effort on these questions. The multiple-choice questions provide quantifiable data that are easy to analyze and review before class. Although they do not contain the richness of student explanations and responses, they can present potentially difficult concepts as an effective gauge of student preparedness and current level of comprehension. Sample question (2) from the microscopy module (Fig. 2.25) probes student understanding of ray optics, converging lenses, and the focal plane. The histogram (next to the question) of student responses shows that students in the course struggled with this concept prior to class. While one can argue that options $\mathrm{C}$ and D are both correct, over two-fifths of the students chose the incorrect options A or B. Consequently, the concept of the focal plane and how it relates to the focal 
(2) What happens to rays that leave a point in the focal plane of a converging lens and emerge from the other side of the lens? Note: The focal plane is the plane corresponding to the focal point. It is perpendicular to the principle axis.

A. the rays will converge at the focal point on the opposite side of the lens

B. the rays will form an image at the focal point on the opposite side of the lens

C. the rays emerging from the converging lens will be parallel to each other

$D$. the rays will form an image at infinity on the other side of the lens
Introduction to Microscopy: Question $2(\mathrm{~N}=64)$

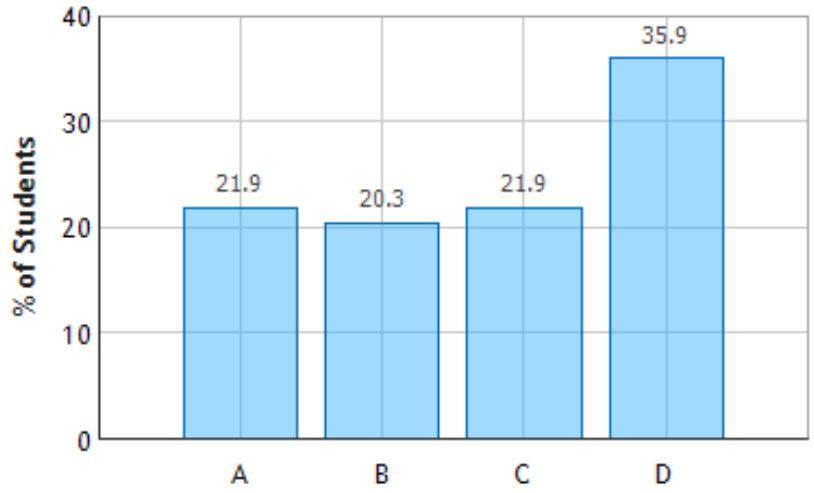

Figure 2.25: Sample checkpoint question and student responses

point and ray diagrams were addressed with greater detail in class.

\subsubsection{Homework Questions}

Homework questions integrate the material, stimulating deeper exploration and engagement from a conceptual standpoint with quantitative calculations and problem solving. These questions were designed to be at a similar level of difficulty as one may find in a traditional introductory algebra based physics textbook. For example, the question set below is from the LASIK module utilizing concepts from thermodynamics (Fig. 2.26).

Some homework questions also require students to read scientific papers before they attempt a solution. This helps improve students' scientific reasoning and ability to take information from one field and apply it to another. 
According to Dr. Teplick, the cornea is mostly water ( $\sim 99 \%$ ). About $0.25 \mu \mathrm{m}$ of corneal tissue is ablated by one laser pulse. This occurs because of the vaporization of water. Take the length of one water molecule to be $\sim 0.1 \mathrm{~nm}$.

(4) The relative power of the Excimer laser is $60 \mathrm{~mW}$. The length of a pulse varies by laser and application; here, assume a pulse length of $10 \mathrm{~ns}$. What is the energy delivered by one pulse?

(5) The latent heat of vaporization of water is $2260 \mathrm{~J} / \mathrm{g}$. How many grams of water can one pulse vaporize?

(6) Use the density of water to find the equivalent volume (in cubic meters) for the previous question.

(7) The previous answer is a volume. Assuming the laser removed a cube of material, what is the depth of material removed?

(8) Recall from the third pre-lecture video, LASIK Fundamentals, that each Excimer laser pulse vaporizes about a quarter of a micron of tissue. How does your answer to the previous question compare to this depth?

(9) What are at least three assumptions that went into the calculation above?

Figure 2.26: Sample homework questions

\subsubsection{Multimedia Modules Analysis}

The FIPF was used to host the multimedia content for the introductory and intermediate courses described in chapters 2.3 and 2.4 respectively. The log data recorded by this platform allows for analysis of students' performance on the assignments as well as their behavior when interacting with the material, which can be used to understand how students engage with the materials and improve the content of the course.

Every time students submit an answer to the pre-lecture, checkpoint, or homework questions, a record is made that includes the student's ID number, the student's answer, the correct answer, and a timestamp. We wrote Python code to analyze different aspects of this data to create a number of different plots. For example, the graph in Fig. 2.27 shows a plot of student responses to each homework question in the endoscopy module. The graph displays multiple factors including how many students attempted the question at least once (solid blue), how many students found the correct answer (dashed red), and the average number of submissions per student (dashed green). Given that less than half of the class found the correct answer, it is clear that questions 5, 12, and 22 were questions students struggled with. 


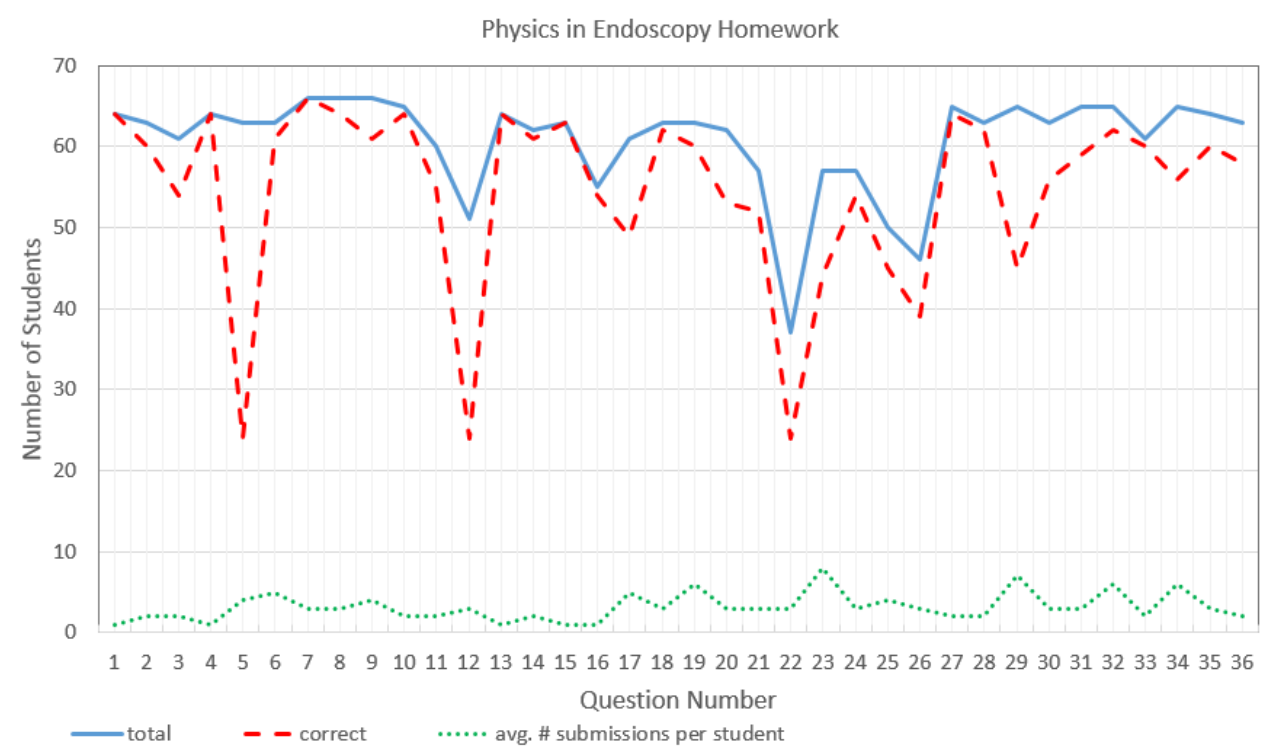

Figure 2.27: Student submissions to the endoscopy module. The plots show the number of students who submitted an answer (solid blue), the number of students who found the correct answer (dashed red), and the average number of submission per student (dotted green).

More detailed examination of these questions can identify concepts that may need to be explored further in-class or in the text. Question number 5 in Fig. 2.27 asks students to find the index of refraction of a medium. The index of refraction is the ratio of the speed of light in a vacuum to the speed of light in a particular medium. Because of this, it will be a unitless value greater than or equal to one. By plotting the frequencies of responses from students, we see that a large number of submissions by students were less than one.

We also explored student behavior and what aspects of the online platform students use. For example, the log data records when students first access a page containing an expert video. Using the difference between that time and the timestamp on their next entry in the log data $(t)$ and the duration of the video $(d)$, we can estimate how many students are watching the entirety of each clip. This will be discussed further in the chapter concerning students' attitudes toward the expert interviews (Sec. 5.2). 


\subsection{Reformed Introductory Physics Course}

The three-term introductory general physics sequences at PSU are divided into two algebra-based sections and two calculus-based sections. While only a small number of life science and pre-health students take the calculus-based sections, the algebrabased courses are dominated by this student group. The pilot course described here reformed one section of the algebra-based course during spring term 2014. The algebra-based physics course covers mechanics in fall term; thermodynamics and electromagnetism in winter term; oscillations, waves, optics, and some modern physics

in spring term. The instruction is fairly traditional, using a standard algebra-based physics text (Walker "Physics" 4th edition) and online homework system [95]. The introductory physics sequence is combined with a mandatory laboratory and elective peer-lead workshop.

\subsubsection{Course Structure}

The course, taught as a pilot over a 10-week period from April to June 2014, met twice a week for sessions lasting 110 minutes each. The 2014 version was offered as online class as well. Applications of the physics topics were introduced through five modules with videos of physicians or biomedical researchers on five different topics: ultrasound imaging, endoscopic surgery, microscopy, LASIK surgery, and pulse-oximetry. Our aim was to cover most of the topics covered in an introductory physics course on waves and optics while emphasizing the application of these topics in modern health fields. Notable exceptions to topics not covered in these video modules were reflection and polarization. These have since been included, but at the time of the course they were covered using a traditional text for three class periods during the first one- 
and-a-half weeks of the course. The length of each module varied, but it took on average one-and-a-half weeks to cover the topics that were addressed in one of the expert videos. The design of the modules was done such that some important topics like lenses and refraction were often covered from different viewpoints in more than one module reinforcing the applicability of physics concepts and problem-solving in various contexts. Some of the modules also drew from concepts that are typically taught early in the introductory physics course sequence. The topics are summarized in Table 2.4. We consider this is one of the course's strengths since it emphasizes that physics concepts have relevance in a wider context; engagement with previously learned concepts reinforces and deepens student understanding.

Table 2.4: Overview of the topics covered in the multimedia modules

\begin{tabular}{|c|c|c|}
\hline Module & Physics concepts & Technical concepts \\
\hline $\begin{array}{l}\text { Ultrasound imaging, } \\
\text { Interview with radiologist }\end{array}$ & $\begin{array}{l}\text { sound, waves, speed of sound, } \\
\text { dB, diffraction, absorption, } \\
\text { Doppler shift }\end{array}$ & $\begin{array}{l}\text { penetration of sound in tissue, } \\
\text { acoustic impedance, shadowing, } \\
\text { 3D imaging }\end{array}$ \\
\hline $\begin{array}{l}\text { Endoscopic surgery, } \\
\text { Interview with } \\
\text { laparoscopic surgeon }\end{array}$ & $\begin{array}{l}\text { blackbody radiation, refraction, } \\
\text { total internal reflection, } \\
\text { standing waves, oscillations, } \\
\text { photons }\end{array}$ & $\begin{array}{l}\text { illumination through fibers, } \\
\text { imaging through fibers and } \\
\text { lenses, harmonic scalpel, digital } \\
\text { detectors }\end{array}$ \\
\hline $\begin{array}{l}\text { Microscopy, Interview } \\
\text { with microscopist }\end{array}$ & $\begin{array}{l}\text { refraction, diffraction limit, } \\
\text { lenses, image formation, light } \\
\text { absorption, fluorescence } \\
\text { (reflection and polarization were } \\
\text { added later) }\end{array}$ & $\begin{array}{l}\text { compound microscopes, confocal } \\
\text { microscopy, time-lapse } \\
\text { microscopy, fluorescence } \\
\text { microscopy }\end{array}$ \\
\hline $\begin{array}{l}\text { LASIK surgery, Interview } \\
\text { with LASIK surgeon }\end{array}$ & $\begin{array}{l}\text { refraction, light absorption, } \\
\text { coherence, lenses, Fourier } \\
\text { analysis, spontaneous and } \\
\text { stimulated emission, latent heat }\end{array}$ & $\begin{array}{l}\text { optical properties of tissue and } \\
\text { water, anatomy of the eye, } \\
\text { myopia, LASERs, aberrometry, } \\
\text { Hartmann-Shack sensor }\end{array}$ \\
\hline $\begin{array}{l}\text { Pulse-oximetry, Interview } \\
\text { with laparoscopic } \\
\text { surgeon/research fellow }\end{array}$ & $\begin{array}{l}\text { light absorption, } \\
\text { electromagnetic spectrum, } \\
\text { spectroscopy, diffraction, Beer's } \\
\text { law }\end{array}$ & $\begin{array}{l}\text { optical properties of water, } \\
\text { tissue, and hemoglobin, two } \\
\text { color pulse-oximetry, } \\
\text { multispectral pulse-oximetry }\end{array}$ \\
\hline
\end{tabular}

The structure of the 2016 version of the course was very similar to the 2014 pilot in many ways. The same modules were used in both courses as well as the semi-flipped format. Group work utilizing learning assistants and weekly quizzes were also shared. 
More detail about the 2016 course will be given in the chapter discussing the research methods.

\subsubsection{Use of Multimedia Modules in the Curriculum}

Using the flipped classroom model, students were tasked with watching the videos presented by biomedical professionals, answering questions, and completing activities on the video content as well as the relevant physics principles in preparation for inclass instruction. Students were also expected to read the course text presenting the core physics and biomedical concepts involved in the videos before attending class. This material was made available to the students through the FIPF as described in chapter 2.2 .

The instructor accessed the responses of the students to biomedical pre-lecture questions and the physics checkpoint questions in the FIPF and used them to guide his teaching. In-class instruction was a combination of lectures (made available to online students after class), discussion, and group work. Following in-class instruction, students used the FIPF for homework, where core physics concepts are applied alongside the connections to the medical field applications. The homework assignments are graded and contributed to the student's final grade.

\subsubsection{Laboratory Activities}

Students enrolled in the spring 2014 pilot course were required to also enroll in a laboratory course that focused on biomedical applications of physics. This laboratory course was only available to the students in the pilot course and not to the students in the traditional physics course. Students meet once a week for 2 hours and 50 
Table 2.5: Schedule of the spring 2014 laboratory section.

\begin{tabular}{|c|c|c|}
\hline Week & Topic & Concepts \\
\hline 1 & $\begin{array}{l}\text { Light Intensity and } \\
\text { Polarization }\end{array}$ & $\begin{array}{l}\text { Power, intensity, polarization, Brewster's angle } \\
\text { (Adapted from the Traditional Lab) }\end{array}$ \\
\hline 2 & $\begin{array}{l}\text { Resonant Frequencies of a } \\
\text { Tube }\end{array}$ & $\begin{array}{l}\text { Wavelength, frequency, open and closed tube resonant } \\
\text { frequencies, wave speed (Adapted from the } \\
\text { Traditional Lab) }\end{array}$ \\
\hline 3 & Endoscopy & $\begin{array}{l}\text { Total internal reflection, acceptance angle, scattering, } \\
\text { coupling }\end{array}$ \\
\hline 4 & Optics of the Human Eye I & Light beams, reflection, refraction, index of refraction \\
\hline 5 & Optics of the Human Eye II & $\begin{array}{l}\text { Lenses, focal lengths, ray diagrams, thin lens equation, } \\
\text { magnification, compound lenses }\end{array}$ \\
\hline 6 & Optics of the Human Eye III & $\begin{array}{l}\text { Common eye defects and corrective lenses, } \\
\text { accommodation, emmetropia, presbyopia, } \\
\text { farsightedness, nearsightedness, aphakia }\end{array}$ \\
\hline 7 & Pulse-Oximetry & $\begin{array}{l}\text { Light absorption, absorption coefficient, wavelength } \\
\text { and frequency }\end{array}$ \\
\hline 8 & Inverted Pendulum Motion & $\begin{array}{l}\text { Potential, kinetic, and mechanical energy, pendulum } \\
\text { period, Froude number }\end{array}$ \\
\hline 9 & Make-Up Week & $\mathrm{N} / \mathrm{A}$ \\
\hline 10 & Lab Exam & $\mathrm{N} / \mathrm{A}$ \\
\hline
\end{tabular}

minutes. There was a total of ten sessions including one session for the final exam and one session in which students had the opportunity to complete an earlier lab that they had missed.

The topics covered by the laboratory course are listed in Table 2.5. The first two weeks of the course consisted of two labs from the traditional laboratory course. These topics were included because they were directly related to the material covered in the pilot course. Details about the reformed laboratory activities can be found in chapter 2.1 . 


\subsubsection{Student Surveys}

A 24-question survey was administered to the students enrolled in the spring 2014 algebra-based life science-focused introductory physics course in an attempt to probe their thoughts on the successes of this pilot course. This survey was administered online, and students received credit for completing the survey. To encourage honest responses, students knew that their answers were reviewed without correlating names to survey responses and not until after final grades were submitted. The questions were primarily short response with a few on a Likert scale from "not useful at all" to "very useful". In accordance with other studies in the physics education research literature, the scoring of the survey was done categorically, rather than ordinally [9]. This means we treat "not useful at all" and "not useful" as the same answer and similarly for "very useful" and "useful". Categorical scoring is considered better than ordinal because students interpretations of something being "very useful" and just "useful" may not be consistent [9].

The data from the short answer questions were sorted into emergent categories of students' responses rather than categories that were determined a priori by the researchers. The category names presented here have been chosen by the authors as best summarizing the theme of the emergent student response category (e.g., Better, No Impact, Worse; More, Same, Less). These labels attempt to summarize all of the responses that fell into that category, and thus, in some cases further explanation of the sentiments within a given category may be needed in order to fully understand the students' response. However, the titles do reflect the sentiment of the group of responses they represent (Table 2.6).

Given that a common outcome for algebra-based physics courses is that students 
Table 2.6: Summary of student survey. In total 68 students submitted the survey. The number of students falling into a particular category is in parentheses.

\begin{tabular}{|c|c|c|c|}
\hline \multirow[b]{2}{*}{$\begin{array}{l}\text { How useful did you find the PDFs introducing the FlipIt } \\
\text { topics? }\end{array}$} & 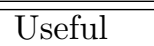 & Neutral & Not Useful \\
\hline & $78 \%(53)$ & $16 \%(11)$ & $6 \%(4)$ \\
\hline How useful did you find the FlipIt Physics pre-lectures? & $73 \%(50)$ & $21 \%(14)$ & $6 \%(4)$ \\
\hline $\begin{array}{l}\text { How useful did you find the FlipIt Physics checkpoint } \\
\text { questions? }\end{array}$ & $37 \%(25)$ & $41 \%(28)$ & $22 \%(15)$ \\
\hline How useful did you find the FlipIt Physics homework? & $90 \%(61)$ & $4 \%(3)$ & $6 \%(4)$ \\
\hline \multirow[b]{2}{*}{$\begin{array}{l}\text { Did the videos on biomedical applications help you see } \\
\text { the relevance of physics in the biomedical field? }\end{array}$} & Yes & I don't know & No \\
\hline & $96 \%(65)$ & $1 \%(1)$ & $3 \%(2)$ \\
\hline \multirow[b]{2}{*}{$\begin{array}{l}\text { Did the biomedical focus make the course better, worse, } \\
\text { or had no significant impact? }\end{array}$} & Better & No impact & Worse \\
\hline & $85 \%(57)$ & $14 \%(9)$ & $1 \%(1)$ \\
\hline \multirow[b]{2}{*}{$\begin{array}{l}\text { Compared to previous physics courses, how much time } \\
\text { do you spend studying for the course over the term? }\end{array}$} & More & Same & Less \\
\hline & $52 \%(35)$ & $43 \%(29)$ & $5 \%(3)$ \\
\hline
\end{tabular}

do not see physics as pertaining to their life science and pre-health disciplines, we wanted to probe to what extent the pilot course impacted their ability to see physics as relevant to coursework in their major. We were also interested in the extent to which students felt that the pilot course was an improvement upon traditional physics courses. As it was the third term in a three-term sequence, students had previously taken two traditional algebra-based physics courses before the pilot course. The student response was overwhelmingly positive. When asked whether the videos on biomedical applications helped them see the relevance of physics in the biomedical field, $96 \%(\mathrm{~N}=65)$ of students responded with "yes". Additionally, when asked about the impact of the biomedical focus, $85 \%(\mathrm{~N}=57)$ considered this focus as an improvement to previous courses.

The small sample size from one iteration of the one-quarter pilot course and the self-reported nature of the data make it difficult to draw final conclusions, although these initial results were encouraging. The response on whether the biomedical focus makes the course better further strengthens the argument that this type of course 
would be seen as beneficial for a broad population of students. The survey results also indicated that the course increased the time students engage with the materials outside of the classroom. Over half of the students responded in the survey that they spent more time on this course than they had in each of their previous two terms in the introductory physics course sequence. Forty-three percentage $(\mathrm{N}=29)$ stated that they spent about the same time on the course, and only three students stated that they spent less time on the course. Despite the increase in studying time, students still reported that the biomedical focus was an improvement from previous introductory courses.

It is also informative to gauge the student's reactions to the individual components of the course. The survey results indicate that in general the students liked the structure in which the material was presented and felt that the tools implemented in the presentation were useful. The checkpoint questions received a more mixed review with $37 \%(\mathrm{~N}=25)$ of the students responding "useful", 41\% ( $\mathrm{N}=28)$ responding "neutral", and 22\% ( $\mathrm{N}=15)$ responding "not useful". The checkpoints were also the lowest rated aspect of the course in the 2016 version. A review of these questions and the way they are presented to students should be a goal. We consider these questions as valuable in helping the instructor identify common misconceptions, gauging the level of understanding before classroom instruction and helping to shape the focus of in-class instruction. In order to stimulate discussion among students and identify common misconceptions, these questions were designed to be at a level that is challenging and often above the level of student understanding prior to instruction. For some students lacking knowledge prior to in-class instruction and the resulting inability to answer these questions could have contributed to students assessing these questions as less useful. Open-ended essay-type questions may compound this effect; however, more investigation is warranted. Motivating and setting the expectations 
for these questions better at the beginning of the course may have an impact on students' perception of these questions.

\subsubsection{CLASS Analysis}

Students were asked to take an online version of the physics Colorado Learning Attitudes about Science Survey (CLASS) multiple times during the 2013-2014 school year [9]. The CLASS scores students on a scale of novice-like to expert-like opinions. Past analysis of CLASS data shows traditional teaching practices composed primarily of lectures have a detrimental impact on all of the eight categories of beliefs the CLASS tests. [96] Research also shows that teaching practices aimed at explicitly addressing student beliefs about physics can have measurable positive effects. [96] More details about the CLASS are given in the methods section (Sec. 3.3.1).

A matched sample of 36 students from the pilot course surveyed at the end of winter term and again at the end of spring term showed a favorable shift of $7.8 \%$ with a standard error of $2.5 \%$ in the overall category $(\mathrm{O})$ (Fig. 2.28). Notably, those students taking the spring pilot course saw a favorable shift of $8.8 \%$ with a standard error of $3.5 \%$ in the category of sense making/effort (SME) and $15.5 \%$ shift with a standard error of $4.24 \%$ in the category of personal interest (PI). A matched sample of 34 from the concurrent traditional spring course was also analyzed. The results from this group showed few statistically significant shifts toward or away from expert-like thinking.

Taken together with the student survey, the responses suggest that instruction in the pilot life science-focused introductory physics class may improve students' beliefs

about physics and physics learning and makes introductory physics more relevant. However, the number of matching students limits the strength of the results as we 


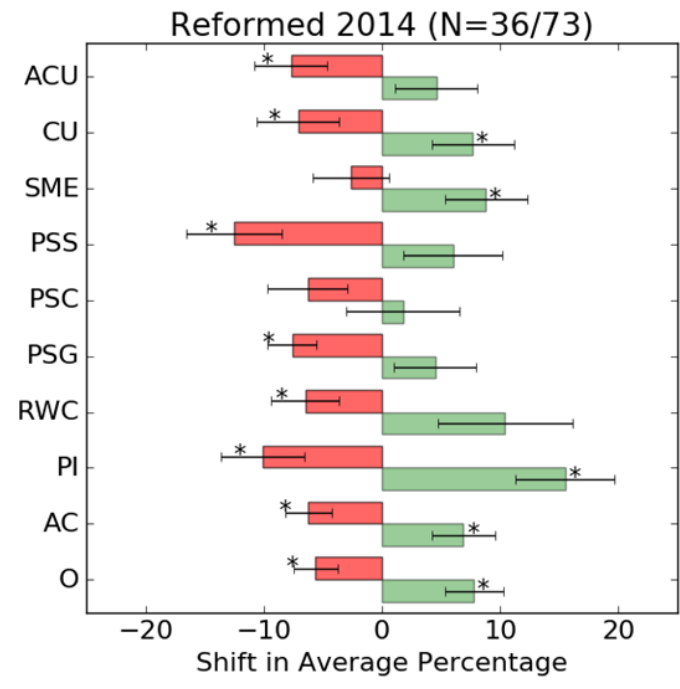

(a)

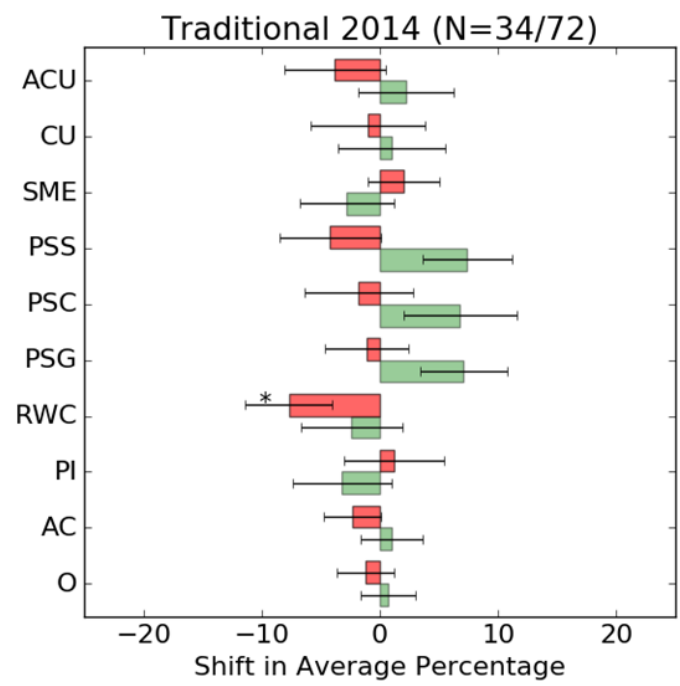

(b)

Figure 2.28: Shifts of matched student CLASS responses between post-winter and postspring term 2014. The error bars represent the standard error. The asterisks represent statistically significant data, i.e. a shift that is at least twice the standard error. The category abbreviations are listed in the methods section (Sec. 3.3.1).

were sampling roughly half of the class. Also, this data does not account for the effect the different instructors of the courses may have had on students' attitudes. Furthermore, evidence from other terms suggests that the time of day the course meets could have also played a role. This morning/evening effect will be discussed in a later chapter.

\subsection{Intermediate Level Physics of Biomedicine Course}

The purpose of the Physics in Biomedicine course is to explore the theoretical foundations of common medical technologies and biological processes. It is an intermediatelevel, intensive course taught over 3-4 weeks and has been offered at PSU since 2008. Students taking the course are expected to have already completed introductory 
physics, but are not required to have taken introductory courses in chemistry or biology. The students who take the course come from a number of fields including biology, pre-health, physics, and computer engineering. Instruction consists of lectures, classroom discussions, laboratory activities, guest lectures by biomedical professionals, and student presentation.

\subsubsection{Guest Lectures}

Guest speakers detail how physical principles are utilized in a practical day-to-day basis. The speakers are members of the local biomedical community, often physicians, biologists, or medical researchers, and cover a wide range of specialties. Some of the specialists who have been invited to speak to the class include radiologists, gastroenterologists, bionutritionists, opthamologists, medical physicists, and microscopists. Many of these speakers were later interviewed for the videos used in the multimedia modules (see chapter 2.2).

\subsubsection{Use of Multimedia Modules in the Curriculum}

The multimedia modules are used in the intermediate course in the same way they are

used in the introductory course. Students are given access to the materials through the FIPF where they view the expert interviews and work through homework questions. Topics that have been covered include body composition analysis, endoscopy, ultrasound, radiation therapy, positron emission tomography (PET), and Magnetic Resonance Imaging (MRI). 


\subsubsection{Laboratory Activities}

The laboratory sessions of the course reinforce the concepts the students learn through the lectures and guest presentations and give students the opportunity to have handson experience with simplified versions of different medical technologies. Many activities used in the course were developed at PSU including the laboratories on bioelectrical impedance analysis, computed tomography, and pulse oximetry, all of which are described in chapter 2.1. Additional activities include equipment such as the 3D educational computed tomography scanner DeskCAT [97] and interactive computer simulations of lasers and MRI scanners [98].

\subsubsection{Student Presentations}

At the end of the course, students present their research of a biomedical topic or technology of their choice. These presentations usually consist of a review of the topic including the relevant physical concepts. Since the class was first offered, the format of the presentations has changed. For many years students participated in a poster session open to the public at the Oregon Museum of Science and Industry. Most recently, students presented their research to their classmates and other stu-

dents through an Ignite Talk [99], in which the speaker must confine their talk to 20 powerpoint slides. the Ignite Talk also included question and answer sessions with small groups of three to five students. These slides are programmed to advance every 15 seconds giving the students 5 minutes to complete their presentation. 


\subsubsection{Pre/Post Content Quizzes}

In order to assess the learning gains students achieve during the introductory or intermediate courses including the laboratory activities, we have developed a number of pre/post content quizzes. This process began by identifying learning goals for a particular topic and designing questions that probe students' understanding of those goals. The questions that probe students' mastery of these learning goals are designed to be open-ended rather than multiple choice or short answer. While this does make the analysis of student responses more difficult to compile, the more restrictive question formats like multiple choice limit students' responses and may not give an accurate depiction of what they have retained. Open-ended questions also allow for verification that the question is understood by students as it was intended. Students are encouraged to respond "I don't know" if they don't know the correct answer. A five-point scale is also included under each question that asks students to rate how confident they are with their answer. An example of this process is given below.

The sixth learning goal from the CT lab (see section 2.1.2) is:

Compare and contrast the energy and atomic interactions of x-ray light to lower spectrum light.

The open-ended prompt that assesses students' improvement on this learning goal is:

Explain why x-rays are less attenuated by tissue as compared to visible light and explain the biological dangers and technological challenges to producing x-ray radiation.

This prompt was given to 18 students in the 2013 intermediate course before and after the CT lab activity. Students were expected to answer that the difference in 
energies of $\mathrm{x}$-rays and visible light is the reason for the difference in attenuation. Furthermore, students should have been able to identify the risks associated with exposure to x-ray radiation.

The results of analysis on this question show that of the nine students that replied "I don't know" in the pre-test, only two replied "I don't know" in the post-quiz. There was a moderate increase in the number of students who identified the correct relationship between the energy levels of visible and x-ray radiation (1/18 pre, $5 / 18$ post) and a large increase in the number of students whose answers indicated the dangers of x-ray radiation (4/18 pre, 11/18 post). Results from quizzes such as this one were used to modify course materials that were potentially unclear. However, it is beyond the scope of this presentation and will not be discussed in detail here. 


\section{Research Methods}

This chapter provide details about the structure and procedures used in the study of the 2016 version of the course. The traditional and reformed courses are discussed as well as confounding factors that might have impacted the results. The assessment methods including surveys, quizzes, and interviews are also described.

\subsection{Description of Courses}

The algebra-based introductory physics sequence at PSU consists of three terms of instruction: fall, winter, and spring. A morning and an evening section of the courses are offered each term. In the 2015-2016 school year, the same instructor taught all six courses of the algebra-based introductory physics sequences. The two sections of the fall and winter term courses did not significantly differ pedagogically because they were taught by the same instructor who used the same lecture materials for both classes. These fall and winter courses used traditional physics content without including a significant amount of biomedical content. The evening section of spring term followed the same format as fall and winter both in physics content and classroom format. We introduced the reformed biomedical content and active learning format to the morning section of the spring term course. See Section 2.3 for a full description of the course. 


\subsubsection{Potential Confounding Factors}

Under ideal conditions, the only difference in instruction would have been the focus on biomedical content. However, there were some confounding factors that may have impacted the final results. In total, there were 100 students in the reformed course and 50 in the traditional course. Studies of student attitudes as measured by the CLASS have shown that smaller classes tend to have more favorable results [96]. The traditional course followed a format very similar to those of the fall and winter term courses. The reformed course introduced a semi-flipped format including regular group problem solving sessions in class. The traditional assessment method of three tests throughout the term was replaced in favor of weekly quizzes and a final. An additional feature on the reformed course was the adoption of a deliberative democracy module [100], in which student groups must reach a consensus on a realworld policy issue. In the reformed course, the students researched and discussed the ramifications of shifting medical education away from stethoscopes toward hand-held ultrasound devices.

Based on surveys, interviews, and conversations, some students decided to take the reformed course either because of the semi-flipped format or the biomedical focus. This could possibly impact analysis of students' attitudes toward course reforms. Many students described both in surveys and the interviews how they viewed reformed functional aspects of the course such as weekly quizzes versus a midterm. The extent to which these aspects impacted students' attitudes is unclear, as students were split on whether they approved or disapproved of the functional reforms. For example, some students described the weekly quizzes as excessive and stated that they preferred midterm exams. Other students said they preferred the quizzes because it helped them to stay on track and not fall behind. 
There is also some evidence that suggests students' attitudes toward physics could be related with whether they were enrolled in the morning or evening section of the course. This is discussed in the chapters on the CLASS results and students' attitudes. The causes of this are not yet know, though this could have also affected the results of studying reforms in a morning course.

The same instructor taught both the reformed and traditional courses, however the instructor's experience was in teaching traditional physics rather than biomedical technology. Adopting new curriculum is a challenge for any instructor. This is especially true when the material is somewhat outside of their area of expertise. The instructor had done work on the biomedical curriculum, but this was the first term that he had taught a full class on the material. On the other hand, he had multiple years of experience with traditional content.

One of the goals for this course was simplifying the adoption of content by physics instructors that have little to no experience with medical topics. It is thought that physics instructors will more easily adopt content that focuses on medical instrumentation, since technology is a feature common to both physics and biomedicine. The instructor gave a short interview with the researchers at the end of the term. The instructor stated that adopting this material was largely not a problem. Although he did occasionally find it difficult to explain certain concepts. This was usually when students asked questions concerning the real-world implications of different physical scenarios. The instructor noted that this was actually a rare occurrence as students usually focused on the physics concepts rather than the physiology. Watching videos of lectures from the previous instructor of the course was also beneficial. Lecture material developed by the instructor will also be helpful to lecturers who want to adopt this material. Many of the in-class demonstrations the instructor performed 
for the traditional section were the same used in the reformed course.

When asked what the developers could do to simplify adoption for future educators, the instructor suggested that more lecture material should be supplied. We should create powerpoint material for each topic, which clearly explains all relevant topics. More clicker, group-work, quiz, and test questions should be prepared for the instructor, especially questions that have biomedical focus. The questions should focus roughly $10 \%$ on biomedical content. Students commented that not enough biomedical material was included in the graded material, which sends the message that it is not worth studying.

\subsection{Description of Student Cohort}

The two cohorts compared in this study are the students in the reformed morning course $(\mathrm{N}=100)$ and the traditional evening course $(\mathrm{N}=50)$. Some students either did not complete the survey or declined to provide information on certain questions. The number of students who responded to a particular question is included in each figure or table.

Demographic information on the students was collected through online surveys and analyzed. No significant differences were found between cohorts in students' reported age, gender, class standing, future career, or high school physics experience. See Sec. 3.4 for a description of the statistical tests used in this study.

Table 3.1 shows the students' responses to the survey prompt "I identify my gender as...". Students could choose from (a) Female, (b) Male, and (c) Other. The 'Other' category had space for students to write in a response. There was not a significant difference in students' gender, $\chi^{2}(1, N=129)=0.18, p=0.67$. 
Table 3.1: Student responses to the survey prompt "I identify my gender as...".

\begin{tabular}{l|ll} 
& Reformed & Traditional \\
\hline \hline Female & $48(54.5 \%)$ & $24(58.5 \%)$ \\
Male & $40(45.5 \%)$ & $17(41.5 \%)$ \\
Total & $88(100 \%)$ & $41(100 \%)$ \\
\hline
\end{tabular}

Figure 3.1 shows the distribution of students' ages. The average age of students in both courses was approximately equal. However, the range of ages of students in the reformed course was slightly larger.

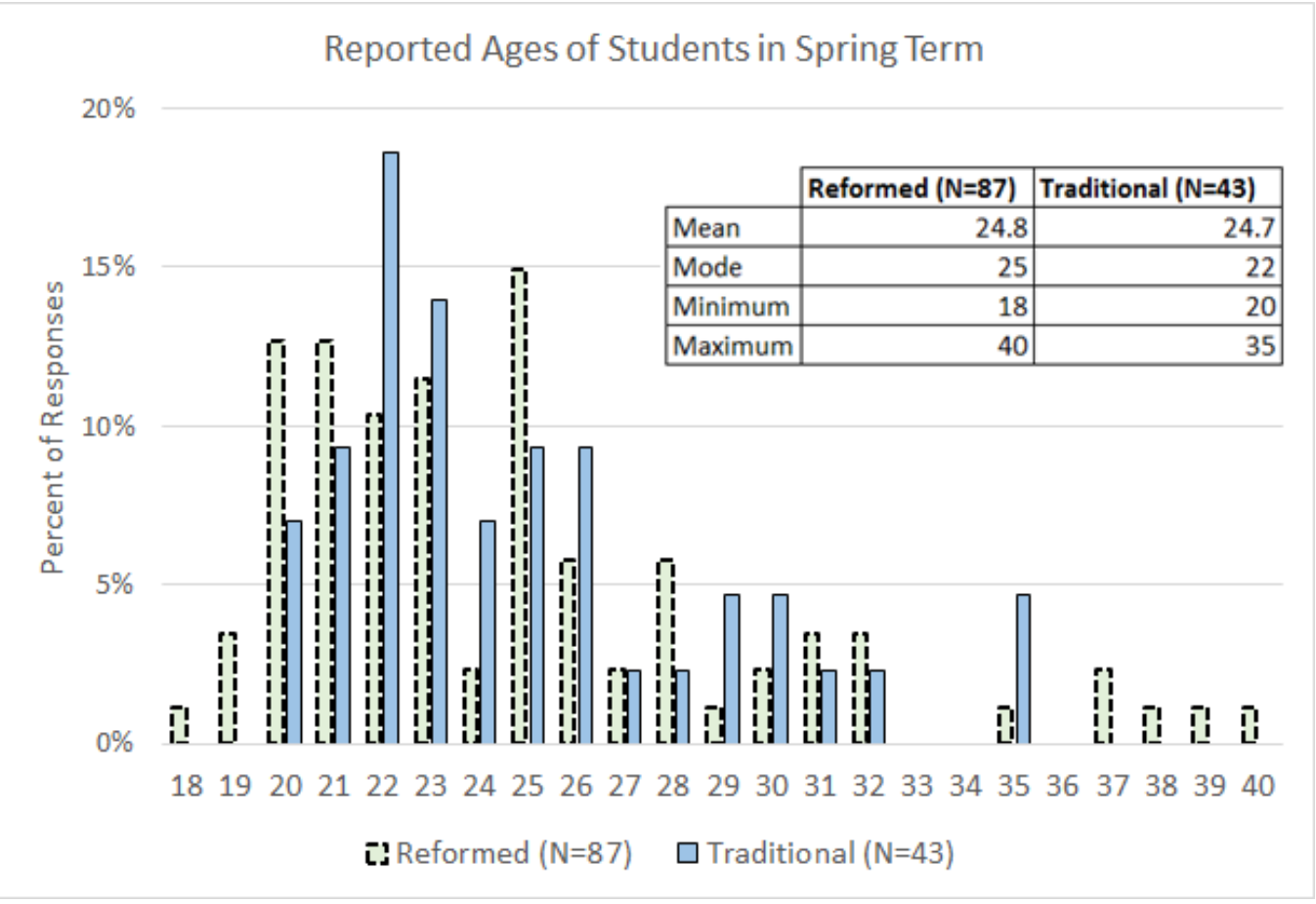

Figure 3.1: Age distribution of students in the reformed morning course and the traditional evening course.

Figure 3.2 shows the class standings of the students in the traditional and reformed courses. The majority of students in both courses were either taking the course at the end of their undergraduate career or as a post-baccalaureate. There was not a significant difference in students' class standing, $\chi^{2}(3, N=129)=1.75, p=0.63$. 


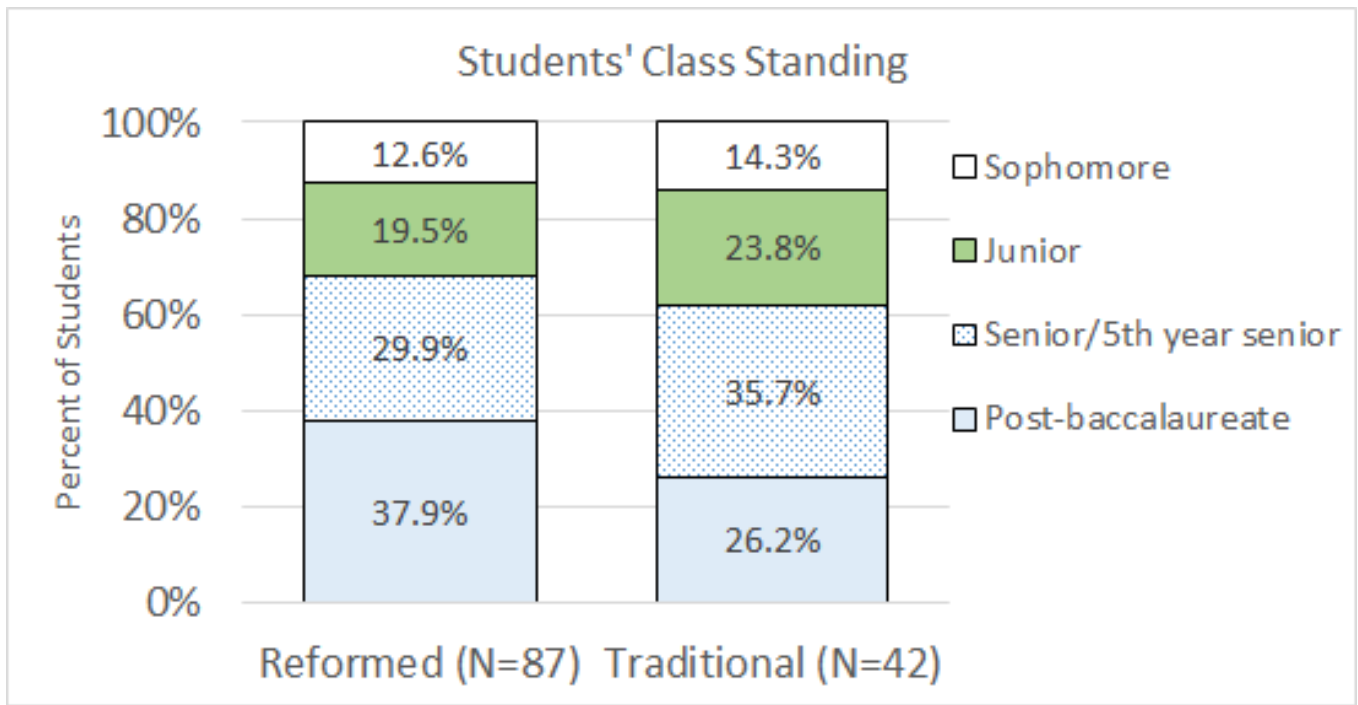

Figure 3.2: Class standing of the students in the reformed morning course and the traditional evening course.

The majority of students enrolled in the algebra-based introductory physics courses at PSU are planning on pursuing a career in health. Figure 3.3 shows the distribution of students' intended professions. The most common careers mentioned are doctor and physical therapist. There was a greater percentage of non-prehealth students in the traditional course. This was to be expected, because we advertised the content of the reformed spring course at the end of winter. However, there was not a significant difference in students' planned careers, $\chi^{2}(5, N=132)=8.43, p=0.13$. Because the focus of the study is on the attitudes of pre-health students, data from some of the students in the 'other' category are excluded from certain analysis. 


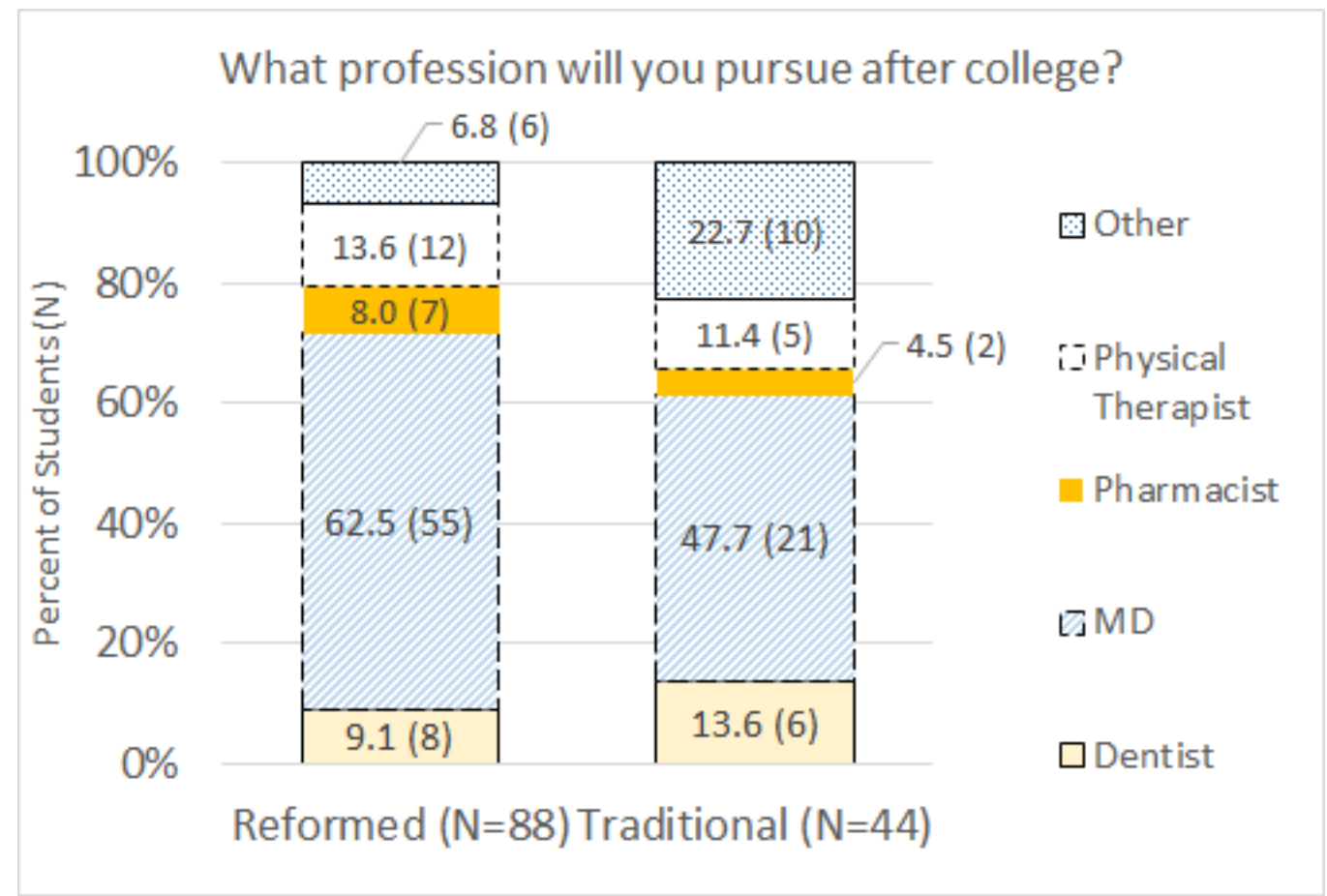

Figure 3.3: Planned professions of the students in the reformed and traditional courses.

In fall term, students were asked what high school physics classes they had completed. Approximately half of the students in both the reformed and traditional courses responded to this question. While many students indicated that they had taken some high school physics, the majority reported that they had taken no physics in high school (Table 3.2). There was not a significant difference between courses, $\chi^{2}(1, N=76)=0.58, p=0.45$.

Table 3.2: Student responses to the survey prompt "What high school physics courses did you take?".

\begin{tabular}{l|ll} 
& Reformed & Traditional \\
\hline \hline None & $37(69.8 \%)$ & $14(60.9 \%)$ \\
Some & $16(30.2 \%)$ & $9(39.1 \%)$ \\
Total & $53(100 \%)$ & $23(100 \%)$ \\
\hline
\end{tabular}




\subsection{Assessment Methods}

The structure of this study is very much in keeping with the assessment methods of similar courses $[28,33,58]$. The researchers who developed those courses focused largely on a qualitative analysis of the ability of students to make conceptual connections between physics and the biological context as well as qualitative and quantitative measures of student attitudes and beliefs.

This research does differ from those studies in that no validated concept inventory was used. In place of a concept inventory, original illustration-based quizzes were given to the students. The reasons for this will be discussed later in this section.

This study also differs in its biological focus. The previous studies were reforms that highlighted how physics is related to biology not biomedicine. However, this study will explore what impact instruction with a biomedical context has on conceptual understanding and attitudinal shifts.

The assessments used in this study will be a mixed methods approach consisting of the Colorado Learning Attitudes about Science Survey (CLASS), original surveys, in-class conceptual problems, and student interviews.

\subsubsection{CLASS}

The Colorado Learning Attitudes about Science Survey (CLASS) is a validated tool to measure students' attitudinal shifts. [60] It consists of 42 Likert questions that are grouped into multiple categories such as 'real wold connection' and 'personal interest'. Responses to these questions are rated as favorable or unfavorable as compared to expert-like responses. For example, strongly agree would be a favorable, expert-like 
response to the statement, "I enjoy solving physics problems."

The CLASS has been used by IPLS researchers to measure shifts in students' perceptions of physics as a result of course reforms. $[28,58,101]$ In these studies, researchers found that the reformed physics courses with a focus on a biological context either improved students' attitudes or resulted in no significant shifts. The fact that attitudes did not show negative shifts toward non-expert like responses was viewed favorably by the researchers. This is because traditional instruction resulted in increases in non-expert like responses. [28,58]

Another attitudinal assessment tool, the Maryland Physics Expectations Survey (MPEX) [61], has also been used by IPLS researchers to measure the impact of curricula reforms. $[8,33]$ These studies also found IPLS reforms resulted in either gains in favorable responses or no significant change.

We chose to employ the CLASS rather than the MPEX for two reasons. Firstly, the CLASS is widely used among physics education researchers [96]. Secondly, the CLASS was used in the studies that most closely resemble our research, namely effects of IPLS reforms on students' interest $[28,58,101]$.

\subsubsection{Analysis}

CLASS results were analyzed using the spreadsheet provided by the developers [102]. Future iterations of the stud will utilize the automatic analysis provided by the PhysPort website [103]. These results are presented as the average percentage of favorable or unfavorable responses per student. The developers defined significant shifts as being twice the standard error of that average. We have adopted this measure of significance for this study. In every analysis presented here, only data from matched 
students were used.

As it will be shown later, students in the reformed and traditional courses entered spring term with different pre scores. Although not specifically prescribed by the developers, the effect size has been used to account for the differences in CLASS pre and post scores between cohorts [104]. The effect size normalizes gains in the average score by the pooled standard deviation, which helps account for differences in cohort size and variations within each group [105].

The effect size was used for the shifts in each CLASS category of the spring term analysis in this study. This was done using sing Cohen's $d$, which is defined as

$$
\frac{<\text { post }>-<\text { pre }>}{s}
$$

where $s$ is the pooled standard deviation of both pre and post averages. The standard categories for effect size were also used: 0.2 small, 0.5 medium, and 0.8 large [105,106].

\subsubsection{Rewritten CLASS Questions}

This study is primarily concerned with the impact course reforms had on students' real-world contextualization of physics concepts and whether that has an effect on their interest in the material. As such, we were most interested to study shifts in the 'real world connection' and 'personal interest' categories of the CLASS.

Although the CLASS is often used to gauge attitudinal impacts of IPLS reforms, there are concerns that it's questions do not probe students' attitudes between physics and biology. [58] There was similar concern about measuring students' attitudes about physics and biomedicine in this study. To address this, questions from the 'real world connection' and 'personal interest' categories of the CLASS were rewritten to focus 
on students' future careers in the health sciences. The rewritten questions were given to students in addition to the original questions. The modified questions will be discussed in more detail in Sec. 4.4.

\subsubsection{CLASS History at PSU}

The primary concern of this study is the 2015-2016 school year. However, the CLASS has been given to all students in the algebra-based physics sequence at PSU since 2012. These results were not analyzed in detail, but are occasionally referred to in this study. Therefore, all CLASS results from the introductory courses are included in the appendix (Sec. A).

\subsubsection{Course Surveys}

Surveys were given to all students in the introductory algebra-based physics courses at the beginning of the school year and again after each term. The surveys consisted of the CLASS as well as demographic and additional attitudinal questions. Some questions were modified from the survey of the 2014 version of the reformed course or from similar studies. [28]

In addition to the CLASS, multiple questions were repeatedly asked throughout the year to measure shifts in students' attitudes. For example, students were asked at the end of every term whether they think physics should be requirement for their major or graduate program. These questions were meant to be more objective measure of changes in students' attitudes rather than relying solely on questions only posed at the end of the reformed course.

The surveys were administered online and consisted of open-ended, multiple choice, 
yes/no, and Likert-style questions. Each Likert-style question had a five-point scale. The most commonly used scale in this survey was the one used by the developers of the CLASS, which ranged from strongly disagree to strongly agree. Original scales had to be adopted for certain questions to maintain clarity. Care was taken to maintain a similar style as the scale used in the CLASS. These original scales will be described along with the results as necessary.

Students were informed at the beginning of every survey that their participation was voluntary. They were told that they would receive extra credit points regardless of how or if they answered the questions. The introduction to each survey also made it clear that the instructor would know who participated so points could be assigned, but would not have access to the data. The instructor would only know the group results, not individual's responses.

Not every question that was asked in the surveys will be discussed here. Some questions probed issues not under review by this study. Other questions did not reveal meaningful data. However, the full list of questions from each survey can be found in the appendix (Sec. B).

\subsubsection{Student Interviews}

The purpose of the interviews was to explore attitudes toward the instruction reforms and probe conceptual understanding using the illustration quizzes. Due to time limitations of the period of research, only students from the reformed course were interviewed. 


\subsubsection{Recruitment}

I visited the classroom on two occasions to recruit students. During those visits, I explained the purpose and structure of the interviews. Students were told that the interviews were voluntary and anonymous. No compensation was offered for participation in the interviews. All students were given slips of paper to indicate whether they would be interested in participating, which students placed in an envelope at the front of the room as they left class. The students who expressed interest were contacted though email and given more information about the interviews as well as access to a scheduling form. The consent form interviewees signed is included in the appendix (Sec. F).

\subsubsection{Procedure}

The interviews were conducted in the week eight of the ten-week term. Two interviewers were present for each session, Elliot Mylott and Warren Christensen from North Dakota State University. Christensen acted as the primary interviewer, directing the pace and content of the interview. Mylott acted as the biomedical content expert, offering leading questions, when appropriate, to illicit further information about the student's conceptual understanding. The interviews were recorded using a video camera, with the except of one student who would only consent to audio recordings.

The interview protocol was split into four main areas: background, conceptual, attitudinal, and course related questions. The background questions ease the student into the interview and provide information about the student's career plans. During the conceptual section, students are asked to complete one of the illustrated concep- 
tual questions. Students were encouraged to vocalize what they think as they completed the question. The attitudinal questions focused on students views of physics and physics instruction. Many of these questions were modified from the course survey. This was done in order to better understand students' responses from the survey. Finally, the course specific questions probe the student's views on the reformed course. Students were asked about the functional aspects of the course (e.g. quizzes, home-

work, group work, etc.) and what they thought of the biomedical focus. Many of these questions were also modified from the course survey. The complete interview protocol can be found in the appendix (Sec. E).

\subsubsection{Participants}

Thirteen students participated in the interviews. This was a sample of convenience, as we accepted anyone who was willing to be interviewed during the time window available to us. Because of this, and the small sample size, we must consider whether the students who participated in the interviews are representative of the students in the reformed course. This comparison was made using demographic information supplied by students in the surveys. The same information was used to compare the students in the reformed and traditional courses in Sec. 3.2.

The names of the interviewees used in this study are pseudonyms selected from a list of baby names. Only students' self-reported gender was considered when choosing pseudonyms. Students were not asked to provide ethnic demographic information; therefore ethnicity is not represented by the pseudonyms. Pseudonyms are not meant to be suggestive of any other demographic factor other than gender.

Table 3.3 compares the gender of the interviewees and the students in the reformed course. The distributions of both groups are similar. 
Table 3.3: Genders of interviewees and the students in the reformed course.

\begin{tabular}{l|ll} 
& Reformed & Interviewees \\
\hline \hline Female & $48(54.5 \%)$ & $7(53.8 \%)$ \\
Male & $40(45.5 \%)$ & $6(46.2 \%)$ \\
\hline Total & $88(100 \%)$ & $13(100 \%)$ \\
\hline
\end{tabular}

The students who participated in the interview tended to be older than the students in the reformed course. The average age of the interviewees was 27.5, whereas the average age of the students in the reformed course was 24.8 .

The class standing of the interviewees was slightly more advanced than the whole of the reformed course (Fig. 3.4). No sophomores participated in the interviews.

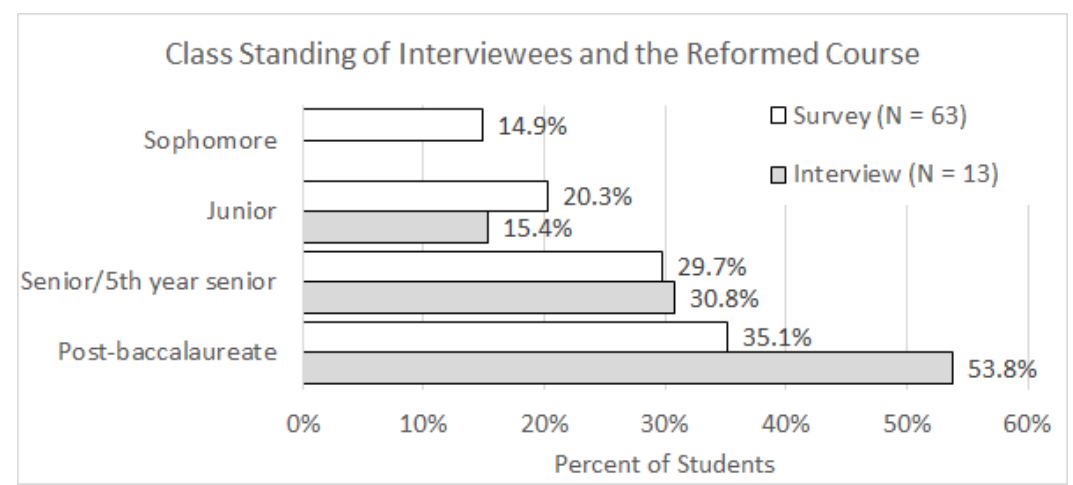

Figure 3.4: Class standing of the interviewees and the students in the reformed course.

Figure 3.5 compares the planned professions of the interviewees and the students in the reformed course. A comparable number of students in both groups plan to pursue an MD. Physical therapists are more prevalent among the interviewees than the whole course. Mo pharmacy or dentistry students participated in the interviews. 


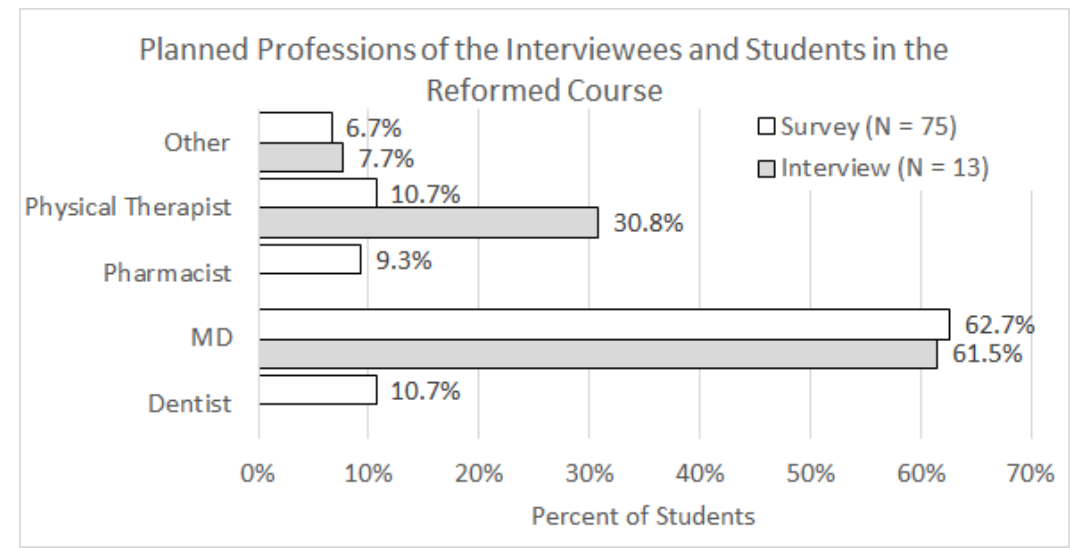

Figure 3.5: Planned professions of the interviewees and the students in the reformed course.

\subsubsection{Illustration Conceptual Quizzes}

The goal of the illustration-based quizzes was to assess whether the biomedical focus of the course reforms impacted students' contextualization and/or conceptual understanding of physical phenomena. The quizzes were open-ended in nature so as not to influence students' responses. The structure, testing procedures, and analysis methods are described in this section.

Other studies of IPLS reforms have relied on validated concept inventories such as the Force Concept Inventory (FCI) [107] and the Force Motion Conceptual Inventory (FMCE) [108] to measure conceptual gains [8,28,33,58,101]. Standard tools like these are extremely valuable and provide a more straight-forward comparison of conceptual understanding to published data. However, researchers have stated that these concept inventories and others like them are not well suited to measure effects of IPLS reforms $[28,43,58]$. Furthermore, concept inventories like the FCI and FCME do not focus on the topics covered in spring term at PSU, which explore waves and light. While there are concept inventories that do focus on these topics $[109,110]$, they were not adopted by this study because they do not assess gain in content knowledge relevant 
to the course reforms.

Another reason to create original questions rather than adopt a standard concept inventory was to maintain an open-ended quality to the prompts. The concept quizzes often explicitly frame the context of the questions, e.g. sound waves from a speaker interacting with a dust particle. A goal of this research was to determine if students' contextualization of physics phenomena is impacted by a biomedical focus. The questions must be as general as possible so as not to influence students' contextualization of the physics concepts.

\subsubsection{Trial Run of Illustration Quizzes}

Prior to spring term, three students were asked to complete the conceptual exercises. Two of these students had taken a traditional physics course and one student had taken the reformed course in spring 2014. The purpose of this trial run was to ascertain whether the assessment would be understood by students and to gauge what kind of responses we might expect to receive. The students responded that the instructions for the assessment were clear and could correctly describe what they needed to do to receive full credit.

Due to time limitations, only two of the four illustration prompts were presented to the students. These two prompts were designed to probe understanding of the Doppler effect and total internal reflection. These illustrations are discussed in Sec. 7.1 and 7.2 respectively. Students were able to correctly identify the intended physical concept

for both illustrations. Based on the students' responses, only slight modifications were needed to improve the clarity of the diagrams.

The trial run was only an informal exploration of the effectiveness of this assess- 
ment strategy. Ideally all four illustration prompts would have been tested with a larger student population. However, limitations of time and resources made wider testing impossible. The trials were useful for verifying the clarity of the instructions and for the slight improvements of the two quizzes tested.

\subsubsection{Test Procedures}

Over the course of spring term 2016, four quizzes were given to students in both the traditional and reformed algebra-based introductory courses. Each quiz featured an illustration depicting a physics concept that was taught in both sections of the course. An example of one of those illustrations is shown in Fig. 3.6. All students were given the same illustrations and the same prompts (Fig. 3.7). All questions were given to students in class as part of a graded quiz (reformed course) or midterm (traditional course). Different point structures were used in the traditional and reformed courses; however the quizzes were worth approximately the same percentage points on the final grade.

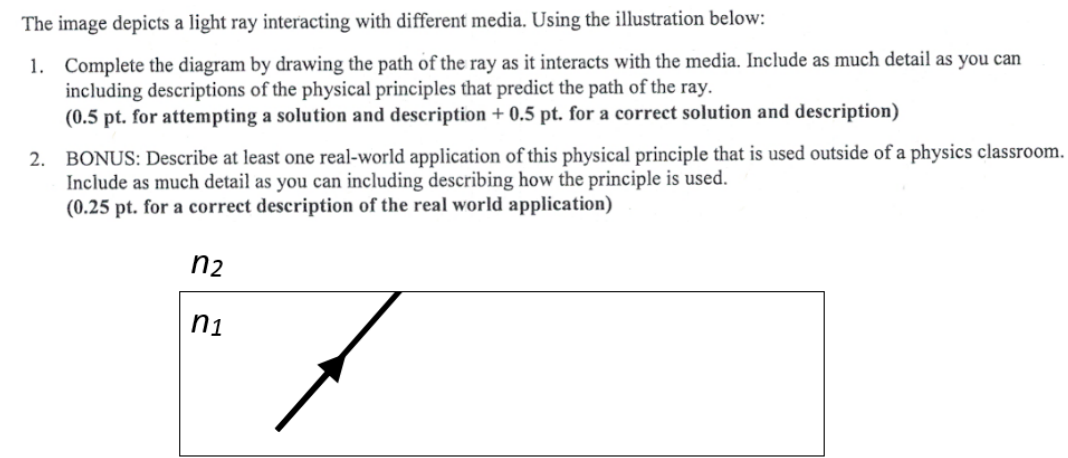

Figure 3.6: Illustration given to students for the quiz on total internal reflection. 
The image depicts a light ray interacting with different media. Using the illustration below:

1. Complete the diagram by drawing the path of the ray as it interacts with the media. Include as much detail as you can including descriptions of the physical principles that predict the path of the ray.

( 3 pt. for attempting a solution and description +3 pt. for a correct solution and description)

2. BONUS: Describe at least one real-world application of this physical principle that is used outside of a physics classroom. Include as much detail as you can including describing how the principle is used.

(1 pt. for a correct description of the real world application)

Figure 3.7: Prompt for the illustrated conceptual quiz on total internal reflection.

\subsubsection{Analysis Methods}

The two main facets of the analysis were of students' conceptual understanding and contextual framing of the different physical concepts represented in each quiz. Each topic was analyzed by two reviewers.

Reviewers created coding templates that captured the emergent trends in student responses. After each reviewer individually applied the coding scheme to the quizzes, the results were compared and templates were altered to better describe the contents of the quizzes. This process was repeated until the reviewers were satisfied that the main trends had been sufficiently detailed. Four reviewers analyzed the data in total: two who rated the trends in conceptual understanding and two who rated the contextual framing.

To limit the possibility of introducing bias into the review process, the quizzes were digitally scanned and made anonymous by cropping out the student signature. Quizzes were randomized and assigned a number. Although many quizzes made reference to biomedical topics covered in the reformed course, the reviewers could not know for certain which section the student was in. 


\subsubsection{Statistical Analysis}

Statistical tests were run on results of the raters' analysis when possible using the chisquare test for independence. Details on the statistical test used are discussed in the next section. There were cases in which statistical tests could not be performed. When prompted to give a real-world application of the physical concept in the illustration, many students gave more than one example. This means that the same student could be represented in more than one of the categories in the dependent variable. Chisquare analysis requires that there is only one observation per student. Discussion of these results is therefore limited to the distribution of students' responses.

\subsubsection{Interviews}

Students who participated in the interviews near the end of term were also asked to complete one of the illustration quizzes. The purpose of including a quiz question in the interview was to probe the reasoning behind certain emergent trends. Students were presented either the Total Internal Reflection or Doppler Shift quiz prompt. These were the only two quizzes that had been administered in both classes at the time of the interviews. Students were asked to complete the illustration and describe their reasoning. Analysis of students' responses are included in the analysis below.

\subsection{Quantitative Statistical Analysis}

Data from this study was analyzed, where appropriate, using statistical tests for independence. The quantitative data in this study was collected through the CLASS, illustrated conceptual quizzes, and student surveys. CLASS data was analyzed using the spreadsheets provided by the developers and is discussed later in this chapter. 
Data from the illustrated conceptual quizzes consisted entirely of coded, categorical variables determined by reviewers based on emergent trends. Survey data consisted of coded open-ended, multiple choice, and Likert-style questions. Likert-style questions are individual questions that follow a Likert type scale, e.g. strongly agree to strongly disagree. These are distinct from Likert questions which are a series of related questions that are analyzed as a whole rather than individually. [111,112] Because of the variability in defining the scale of Likert-style questions, responses were combined into three nominal categories: agree, neutral, and disagree. This practice reduces the possibility of incorrect inferences caused by analyzing the original ordinal scale. $[60,111,112]$ Therefore, all the quantitative data in this study, with the exception of student age, was categorical.

The most appropriate statistical test for the categorical, quantitative data in this study was the Chi-square test for independence. More precise tests such as Fischer's exact test were not appropriate given that contingency tables were often larger than $2 \times 2$. Unless otherwise noted, $p$ values reported in this study are calculated using the Chi-square. The standard alpha value of 0.05 was used as the level of significance. The reported $p$ values in this study are exact.

There are three standard assumptions of the Chi-square test. $[106,113]$ The first is that each variable is independent, meaning a single subject is not included more than once in a contingency table. the second is that counts rather than frequencies are used. And finally, each expected value should be greater than one and no more than $20 \%$ of the expected frequencies should be less than five. There are instances in the data where one or both of these assumptions are not met, particularly in the illustrated conceptual quizzes. These instances will be discussed in turn. Otherwise, data in this study satisfied all three assumptions. 


\section{CLASS}

The CLASS was administered four times in the 2015-2016 school year: (1) pre fall and (2) post fall, (3) post winter, and (4) post spring term. Results from each of these surveys are discussed in this chapter. Because the course reforms happened in spring term, the shifts from post winter to post spring are discussed in more detail. The results of from the entire year and from previous years are included in the appendix (Sec. A).

The results of the CLASS in spring term show an improvement in attitudes from students in both the traditional and reformed courses. Students in the traditional course had larger gains, however students in the reformed course had higher average favorable responses. Analysis of rewritten survey questions suggests that students' attitudes were altered in ways not assessed by the traditional CLASS. Results from the whole year indicate that course reforms early in the year would potentially have had a larger effect than those seen in spring term.

CLASS Reporting The graphs of CLASS results in this section show shifts in both favorable and unfavorable responses. Ideally, we would see positive shifts in favorable responses and negative shifts in unfavorable responses. Asterisks in the graphs represent statistically significant shifts, which are defined by the developers as twice the standard error. 
Developers of the CLASS identified a number of categories that are addressed in the survey [9]. These categories of the CLASS are abbreviated in this chapter according to Table 4.1.

Table 4.1: List of categories in the CLASS and the abbreviations used in this section.

\begin{tabular}{l|l} 
Category & Abbreviation \\
\hline \hline Overall & O \\
All Categories & AC \\
Real World Connection & RWC \\
Personal Interest & PI \\
Sense Making/Effort & SME \\
Conceptual Understanding & CU \\
Applied Conceptual Understanding & ACU \\
Problem Solving General & PSG \\
Problem Solving Sophistication & PSS \\
Problem Solving Confidence & PSC \\
\hline
\end{tabular}

\subsection{Fall and Winter Terms}

This section briefly describes the CLASS results from the two terms before the spring course reforms. This section only represents matched data from a single term. The group of students described in winter are not the exact same group of students described in fall. An analysis of matched students over the full year will be discussed in a later section of this chapter.

Over the course of fall term, students in both courses show an increase in unfavorable responses, though this trend is stronger in the morning section. The results of the matched responses from the pre fall to post fall shifts are shown in Fig. 4.1. The results from the students in the morning section show significant increases in unfavorable responses in every category. Every category also shows decreases in the average percent of favorable responses, most of which are also significant. While the shifts 
from students in the evening course also show increases in unfavorable responses in every category, most shifts are smaller in comparison to the morning section. Both groups show increased unfavorable and decreased favorable responses in both the PI and RWC categories.

The cause of the difference in these results is not known. Both the morning and evening sections were taught by the same instructor, who used the same course structure and materials. This data fits into a trend we have seen over multiple years of administering the CLASS, where students in the evening section tend to have more positive results than the students in the morning section. This will be discussed in the conclusion to this chapter.

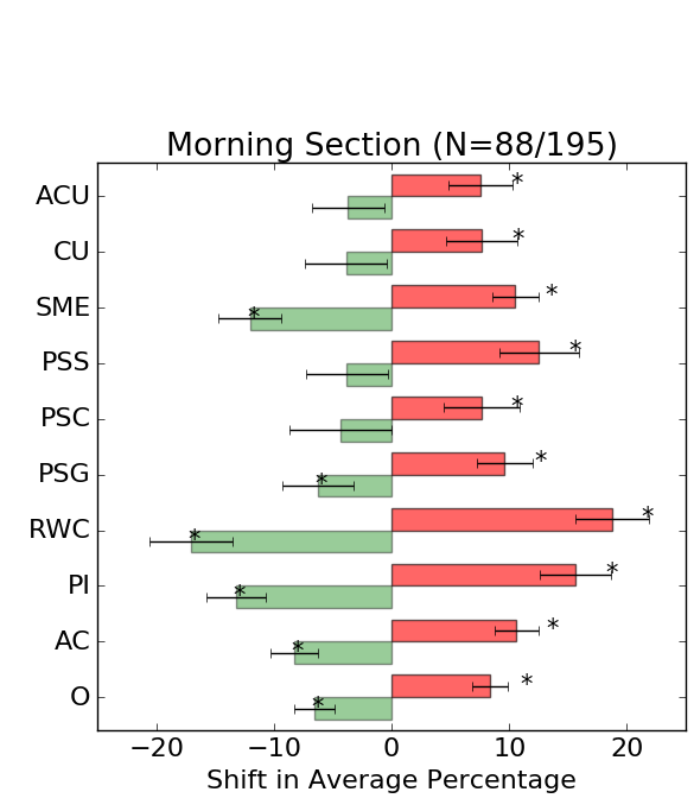

(a)

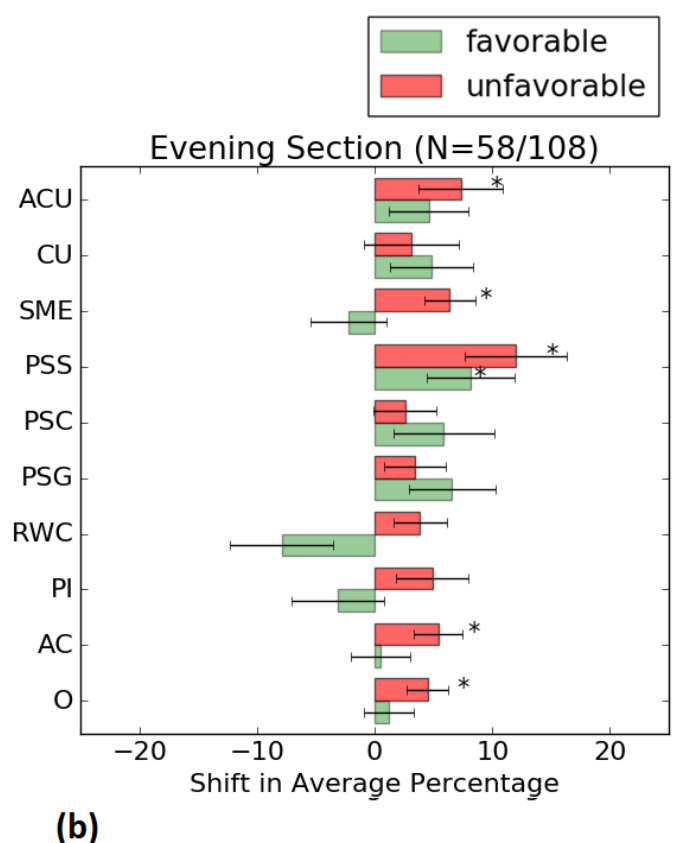

(b)

Figure 4.1: Results of the CLASS administered at the beginning and end of fall term for students in (a) the morning section and (b) the evening section. The asterisks denote statistically significant shifts.

Shift in students' attitude in winter term were more positive than in fall, though generally smaller in magnitude. The results of the matched responses from the post 
fall to post winter shifts are shown in Fig. 4.2. For many categories, we see an increase in favorable responses and a decrease in unfavorable responses. However, these shifts are generally smaller than those seen in fall term. Of all the results, the only significant shift is seen in the unfavorable responses in the PSS category for the morning section. Both groups show improvements in the RWC connection category. On the other hand, the PI category remains relatively unchanged.

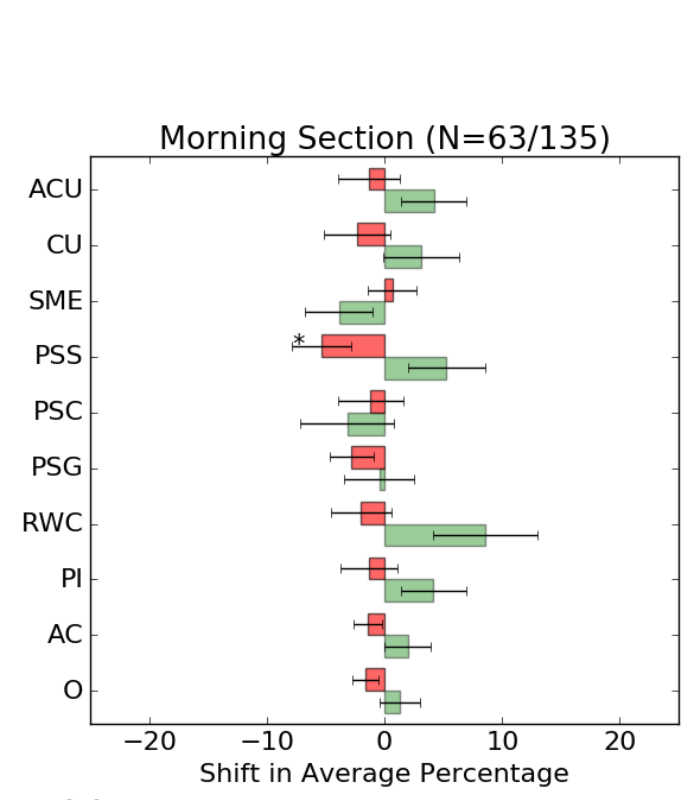

(a)

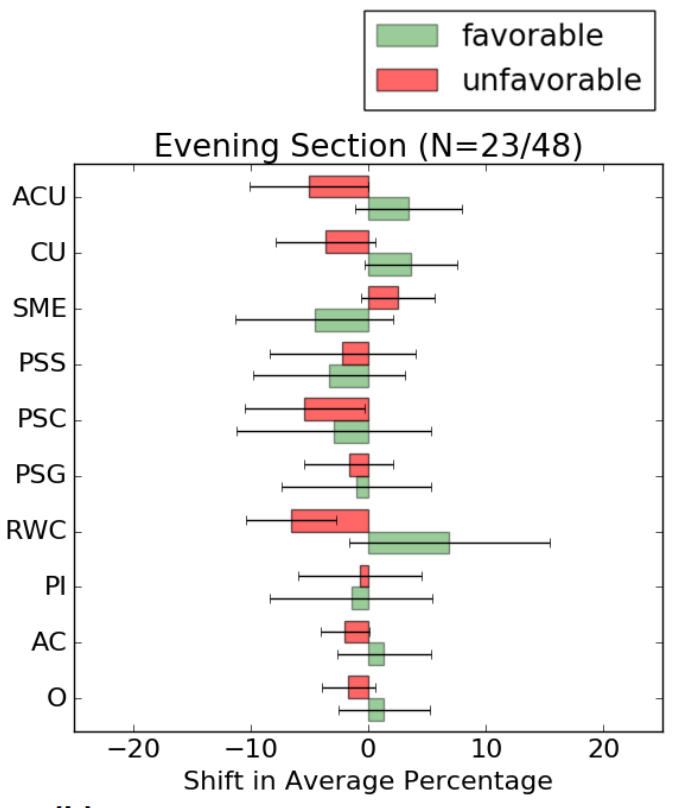

(b)

Figure 4.2: Results of the CLASS administered at the end of fall term and the end of winter term for students in (a) the morning section and (b) the evening section. The asterisks denote statistically significant shifts.

\subsection{Spring Term}

The focus of this study is on the impact of course reforms on attitudes of pre-health students. Therefore, only the results from students who reported pursuing a career in the health sciences are included in the analysis in this section. Because of this one student from the reformed course and seven from the traditional course were removed 
from the initial results. The original results that include these removed students can be found in the appendix (Sec. A).

Figure 4.3 shows the results of the CLASS administered at the end of the winter term and again at the end of spring term. The results of the average favorable responses are also shown in Table 4.2. The students in the traditional course generally demonstrated larger favorable shifts from their post winter responses than the students in the reformed course. Many of the categories show shifts large enough to be considered statistically significant, such as PSS. The students in the reformed course also show generally favorable shifts, but smaller in magnitude.

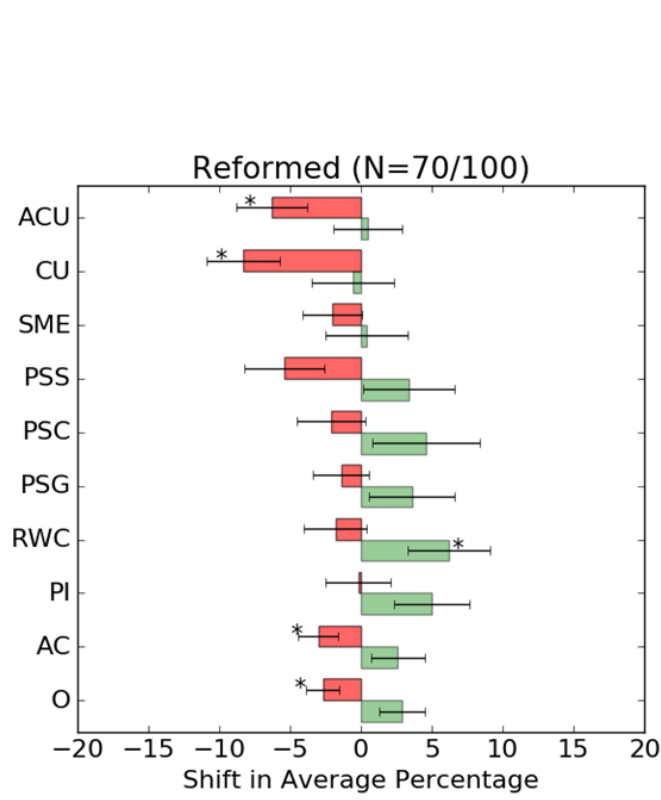

(a)
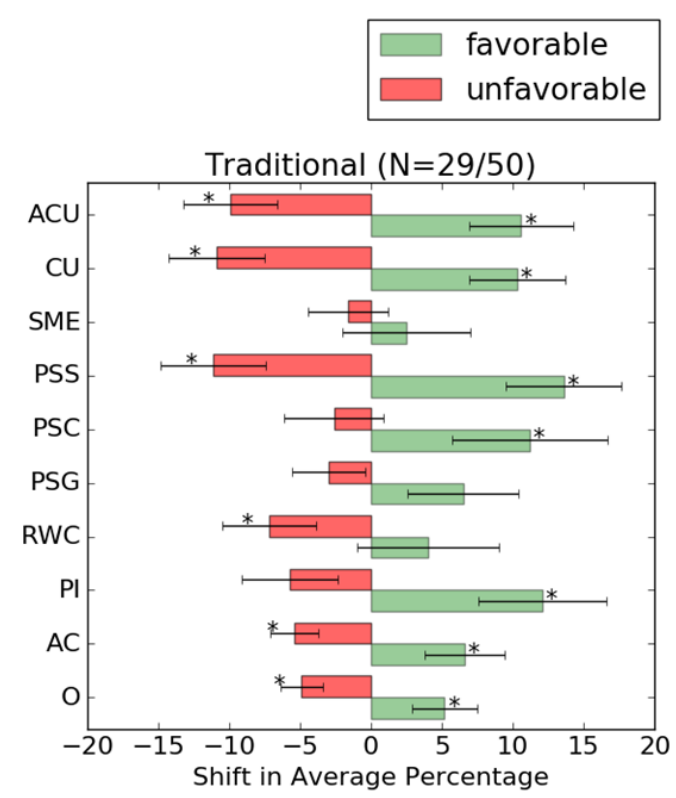

(b)

Figure 4.3: Results of the CLASS administered at the end of the winter and spring terms for students in (a) the reformed course and (b) the traditional course. The asterisks denote statistically significant shifts.

One of the primary goals of the reformed course was to improve pre-health students' interest in physics by explicitly highlighting the real world applications relevant to their future careers. For this reason, both the Personal Interest (PI) and Real World 
Table 4.2: Average percent of favorable responses from students in the reformed and traditional courses. Asterisks denote statistically significant shifts, which are defined as twice the standard error. Pre data came from the post winter survey and post data came from the post spring survey. The effect size (ES) for each category is also given.

\begin{tabular}{l|llllr|lllll} 
& \multicolumn{7}{|c|}{ Reformed Course $(\mathrm{N}=70)$} & \multicolumn{5}{|c}{ Traditional Course $(\mathrm{N}=29)$} \\
\hline \hline Cat. & Pre & Post & Shift & StErr & ES & Pre & Post & Shift & StErr & ES \\
\hline O & 57.8 & 60.6 & 2.8 & 1.6 & 0.16 & 51.2 & 56.3 & $5.2^{*}$ & 2.3 & 0.28 \\
AC & 57.4 & 60.1 & 2.6 & 1.9 & 0.13 & 49.5 & 56.0 & $6.6^{*}$ & 2.8 & 0.31 \\
PI & 58.6 & 63.6 & 5.0 & 2.7 & 0.17 & 49.4 & 61.5 & $12.1^{*}$ & 4.5 & 0.36 \\
RWC & 68.1 & 74.3 & $6.2^{*}$ & 2.9 & 0.20 & 62.4 & 66.4 & 4.0 & 5.0 & 0.12 \\
PSG & 66.4 & 70.0 & 3.6 & 3.0 & 0.14 & 58.6 & 65.1 & 6.5 & 3.9 & 0.27 \\
PSC & 65.4 & 70.0 & 4.6 & 3.8 & 0.15 & 59.5 & 70.7 & $11.2^{*}$ & 5.5 & 0.40 \\
PSS & 42.9 & 46.3 & 3.4 & 3.2 & 0.12 & 35.9 & 49.4 & $13.6^{*}$ & 4.1 & 0.50 \\
SME & 64.7 & 65.1 & 0.4 & 2.9 & 0.02 & 53.7 & 56.2 & 2.5 & 4.5 & 0.10 \\
CU & 54.8 & 54.2 & -0.6 & 2.9 & -0.02 & 40.8 & 51.1 & $10.3^{*}$ & 3.4 & 0.34 \\
ACU & 38.8 & 39.3 & 0.5 & 2.4 & 0.02 & 30.3 & 40.9 & $10.6^{*}$ & 3.7 & 0.39 \\
\hline
\end{tabular}

Connection (RWC) categories of the CLASS are of special interest. The CLASS results in Fig. 4.3 show that students in the reformed course did have a significant shift in favorable responses in the RWC category. However, the PI category shift was not significant. In fact the students in the traditional course show a much larger and significant shift in personal interest.

Despite the smaller shifts of the reformed course, analysis of the pre and post values in each category reveal that students in the reformed course had higher average favorable responses than those in the traditional course. Table 4.2 lists the average favorable percentages in each category from both cohorts. In every category in the pre CLASS data from the end of winter term, the students in the reformed course had a higher percentage of favorable responses than the students in the traditional course. Furthermore, students in the reformed course maintained a higher percentage of favorable responses in nearly every category. This is true of every category except PSC and ACU, where the differences in average post scores was less than $2 \%$. Nevertheless, the effect sizes listed in Table 4.2 indicate that the course reforms had 
little effect on shifts in student attitudes compared to the traditional course. Only the RWC category just barely passes the threshold for the small effect category.

\subsection{CLASS by Gender}

While the course reforms had smaller effects on the students as a whole, the data suggests that women's attitudes were more impacted than men's.

The CLASS results from Fig. 4.3 were divided according to the gender reported by students in the survey (Figs. $4.4-4.5$ ). One student from the traditional course declined to provide gender information. Therefore, this individual's data is not included in this section.

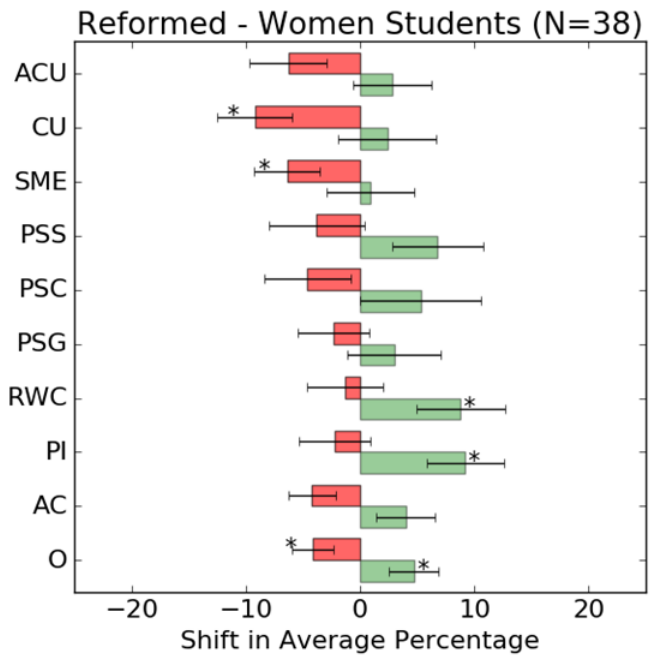

(a)
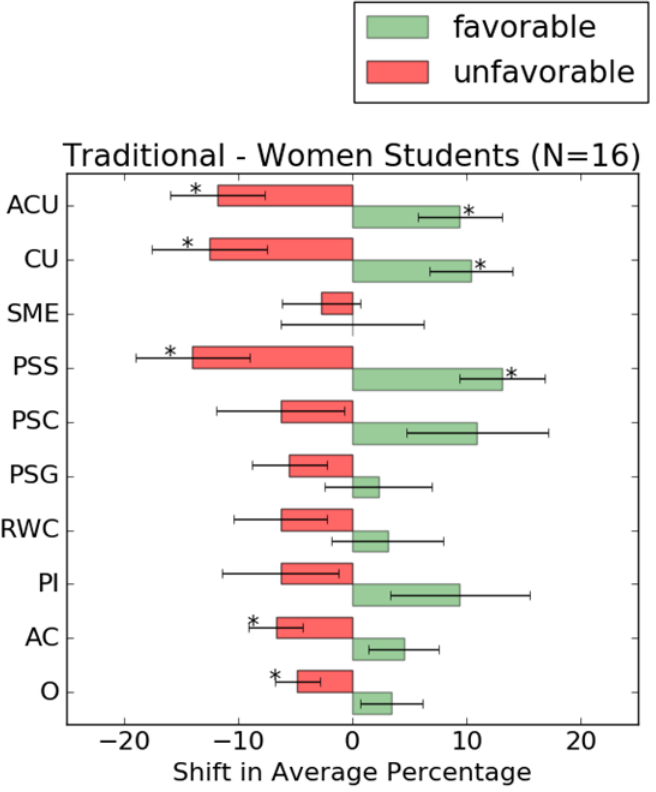

(b)

Figure 4.4: Results of the CLASS for women students in (a) the reformed course and (b) the traditional course. The asterisks denote statistically significant shifts. 


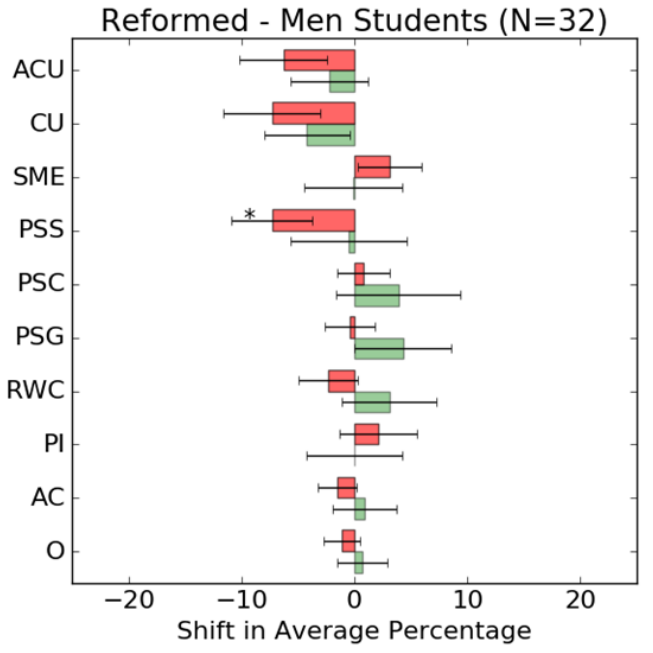

(a)

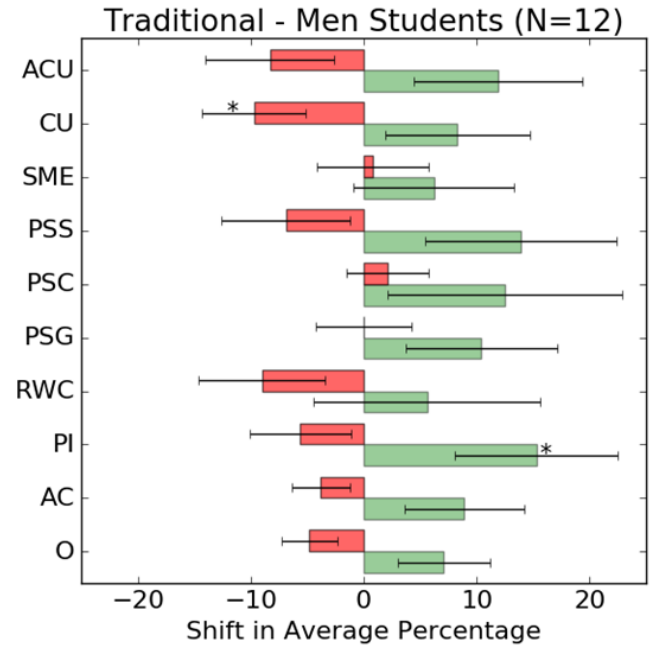

(b)

Figure 4.5: Results of the CLASS for men students in (a) the reformed course and (b) the traditional course. The asterisks denote statistically significant shifts.

Women in the reformed course show increases in favorable responses and decreases in unfavorable responses in every category. This was also true of the students as a whole. However, women also show significantly positive shifts in multiple categories including both the PI and RWC categories. Men in the reformed course, on the other hand, show only one significant shift, a decrease in the unfavorable responses in the PSS category.

\subsection{Career-focused CLASS Questions}

Although the CLASS is a common tool when assessing student attitudes, the tool might not be probing the effect of course reforms on pre-health students' attitudes. The course reforms focused on improving students' attitudes toward the relevance of physics in biomedicine. The CLASS does not directly address this connection through 
the PI and RWC categories, which use phrases like 'everyday life' and 'real world'. The question becomes whether students interpret these terms as having to do with their future careers.

Methods In order to probe this effect, questions from the PI and RWC categories were rephrased to put emphasis on students' future careers rather than the 'real world' or 'everyday life'. The original and rewritten versions of these questions are listed in Table 4.3. Both versions were given to students. These questions were designed to be as similar to the original questions as possible, while still maintaining clarity. Questions 28 and 37 were not rewritten because a suitably concise statement about careers could not be determined.

Table 4.3: Statements from the Personal Interest and Real World Connection categories of the CLASS. Questions in italics are the rewritten versions of the questions, designed to emphasize students' careers.

\begin{tabular}{|c|c|c|c|}
\hline No. & Question & RWC & $\mathrm{PI}$ \\
\hline 3. & $\begin{array}{l}\text { I think about the physics I experience in everyday life. } \\
\text { (I think about the physics that is involved in my future career.) }\end{array}$ & & $\mathrm{X}$ \\
\hline 11. & $\begin{array}{l}\text { I am not satisfied until I understand why something works the way it does. } \\
\text { (When I learn about technology that is used in my future career, } \\
\text { I am not satisfied until I understand why it works the way it does.) }\end{array}$ & & $\mathrm{X}$ \\
\hline 14. & $\begin{array}{l}\text { I study physics to learn knowledge that will be useful in } \\
\text { my life outside of school. } \\
\text { (I study physics to learn knowledge that will be useful in my future career.) }\end{array}$ & & $\mathrm{X}$ \\
\hline 25. & $\begin{array}{l}\text { I enjoy solving physics problems. } \\
\text { (I enjoy solving physics problems that are relevant to my future career.) }\end{array}$ & & $\mathrm{X}$ \\
\hline 28. & $\begin{array}{l}\text { Learning physics changes my ideas about how the world works. } \\
\text { (Not given) }\end{array}$ & $\mathrm{X}$ & $\mathrm{X}$ \\
\hline 30. & $\begin{array}{l}\text { Reasoning skills used to understand physics can be helpful to me } \\
\text { in my everyday life. } \\
\text { (Reasoning skills used to understand physics can be helpful to me } \\
\text { in my future career.) }\end{array}$ & $\mathrm{X}$ & $\mathrm{X}$ \\
\hline 35. & $\begin{array}{l}\text { The subject of physics has little relation to what I experience } \\
\text { in the real world. } \\
\text { (The subject of physics has little relation to what I will experience } \\
\text { in my future career.) }\end{array}$ & $\mathrm{X}$ & \\
\hline 37. & $\begin{array}{l}\text { To understand physics, I sometimes think about my personal experiences } \\
\text { and relate them to the topic being analyzed. } \\
\text { (Not given) }\end{array}$ & $\mathrm{X}$ & \\
\hline
\end{tabular}


The rewritten questions were presented in a stand-alone section of the survey that started with the question, "What is your intended profession after college?". Following this, they were then given the questions modified from the CLASS questions in Table 4.3.

Under ideal conditions, the original and rewritten questions would be given to students at the same time in the same survey. While that was the case for the post question data, it was not true of the pre question data. The original CLASS questions were given at the end of winter term and the rewritten questions were given at the beginning of spring term. This is a difference of approximately three weeks. The developers of the CLASS cautioned researchers to mindful of the effect the winter break can have on shifts in students' attitudes [60]. It is not clear whether this had any effect on students' responses. Future studies should take care to presents both forms of the questions at the same time. Both versions of the CLASS questions were given to students in the same post spring survey.

Results The goal was to determine if students answer these questions any differently than the original versions. Therefore, only the responses from students who answered both versions of these questions in the pre and post surveys are compared. Because this research is focused on the effect of course reforms on students entering a career in the health sciences, only the responses from those students are included here. A total of 63 students in the reformed course and 25 in the traditional course fit this criteria.

The results are shown in Fig. 4.6. Each graph shows the shifts in the average percent of favorable responses for the original and rewritten question. 
Q3. I think about the physics that is involved in my future career.

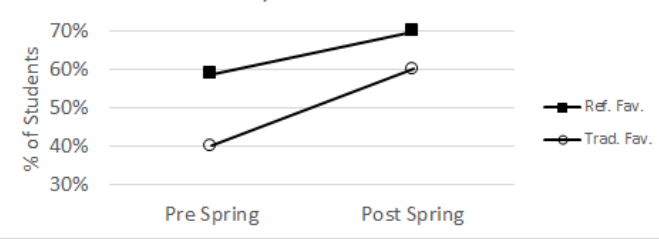

Q11. When I learn about technology that is used in my future career, I am not satisfied until I understand why it works the way it does.

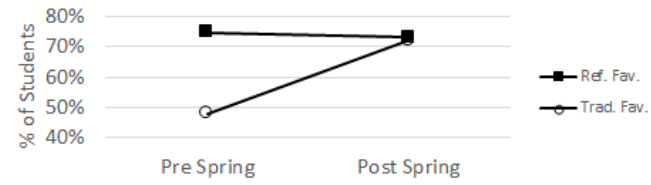

Q14. I study physics to learn knowledge that will be useful in my future career.

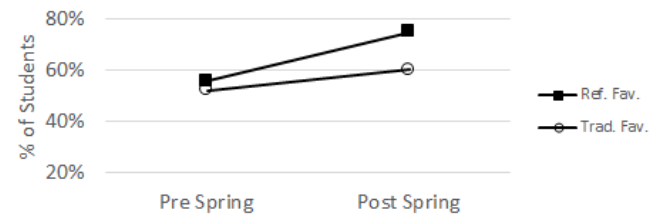

Q25. I enjoy solving physics problems that are relevant to my future career.

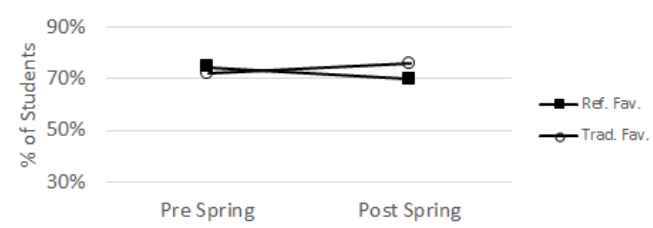

Q30. Reasoning skills used to understand physics can be helpful to me in my future career.

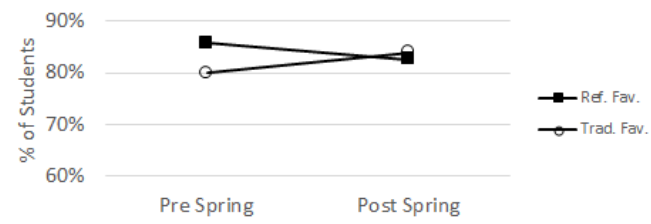

Q35. The subject of physics has little relation to what I will experience in my future career.

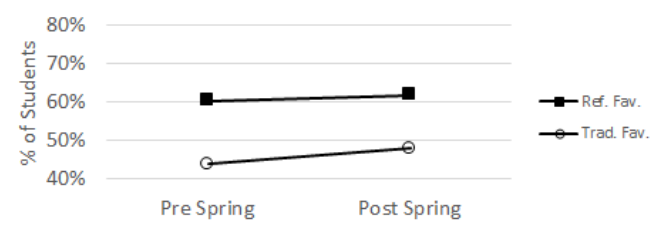

(a)
Q3: I think about the physics I experience in everyday life.

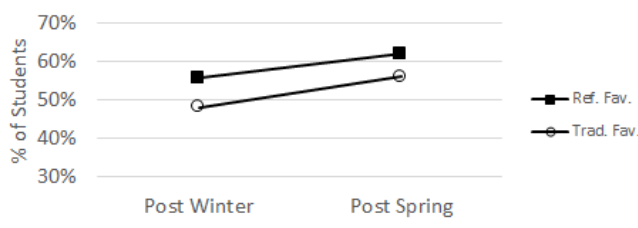

Q11: I am not satisfied until I understand why something works the way it does.

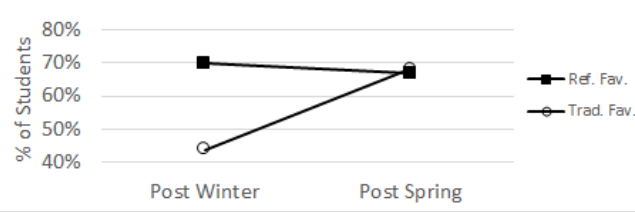

Q14: I study physics to learn knowledge that will be useful in my life outside of school.

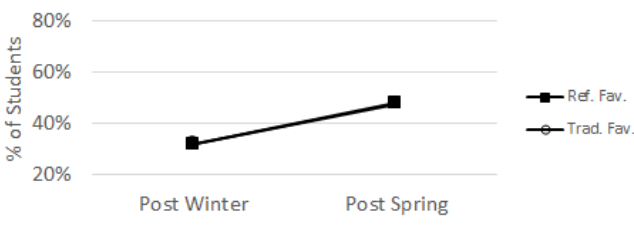

Q25: I enjoy solving physics problems.

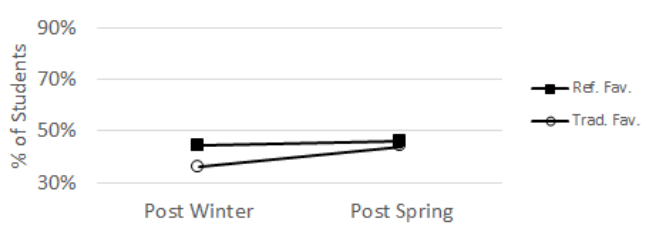

Q30: Reasoning skills used to understand physics can be helpful to me in my everyday life.

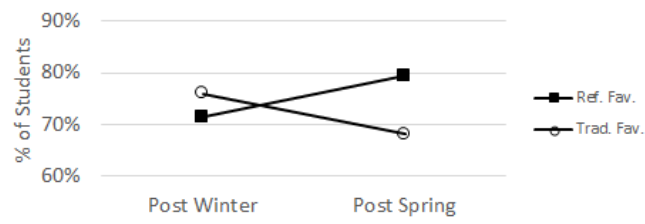

Q35: The subject of physics has little relation to what I experience in the real world.

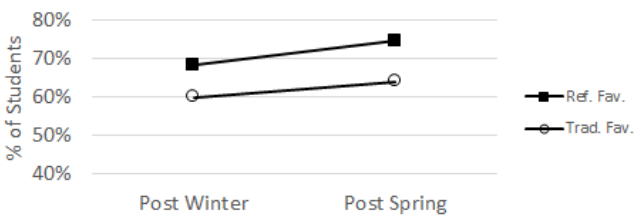

(b)

Figure 4.6: Results of the (a) modified and (b) original version of questions from the CLASS. 63 students from the reformed course (black squares) and 25 students from the traditional course (empty circles) responded to both. 
Because questions 28 and 37 were not reformulated, a direct comparison of the total PI and RWC categories could not be done. Instead the results of each rewritten question were compared to the results of the corresponding, original question.

Students' responses to the different forms of the questions are noticeably different. This is true of each item expect for question 11, which had similar results for both versions. Questions 14 and 30 suggest that students are more likely to view physics as 'helpful' or 'useful' to their careers than their everyday life. Students report in question 25 that they enjoy physics problems more when they are relevant to their careers. Although the students responded that they are more likely to think about the physics in their future careers (Q3), they are less likely to see the relation of physics in medicine than in the 'real world' (Q35).

Interview Responses The question of just how students interpret the phrase 'everyday life' was probed during the interviews. Their responses support the idea that their future careers in the health sciences are not the first thing they associate with the term 'everyday life'.

Students were asked during the interviews, "Do you ever think about the physics your experience in everyday life?". This is almost a direct version of question three from the CLASS and is one of the questions that was rewritten to have a focus on careers (Table 4.3).

Despite the fact that these students had been in the reformed course for seven weeks by the time of the interviews, they did not volunteer medical applications in response to the 'everyday life' prompt. Rather students talked about examples such as the diffraction of water waves at the beach, baseball, and driving cars.

One student, in response to another question, explicitly differentiated medicine 
and 'real life'.

Interviewer: Was that discussed previously in other courses, how things relate to medicine?

Sarah: No, not really. I mean we would talk about like how they relate to real life, but not necessarily anything to medicine in particular.

One student did suggest that they associate medical equipment with everyday life.

The student was being asked about the connection between physics and medicine.

Hailey: I mean, just being able to relate it to real life stuff that's easily accessible or things that people come across in everyday life. I mean, sure we come across currents and circuits and stuff like that. ... I haven't had to deal with them that much. I know that it happens all around me but I'm not actually using that stuff directly. But stuff like how a pulse oximeter works. I use those every time I work.

\subsection{Full Year Analysis}

Responses from each of the four surveys were matched for both courses. This resulted in a total of 46 students from the reformed course and 22 students from the traditional course. These totals include pre-health students and students who are not pre-health. The students are divided in this section according to which course they took in spring term, reformed or traditional.

The shifts in favorable and unfavorable responses to the RWC and PI categories for these students are shown in Fig. 4.7. The results of each category for the academic year can be found in the appendix (Sec. A). 


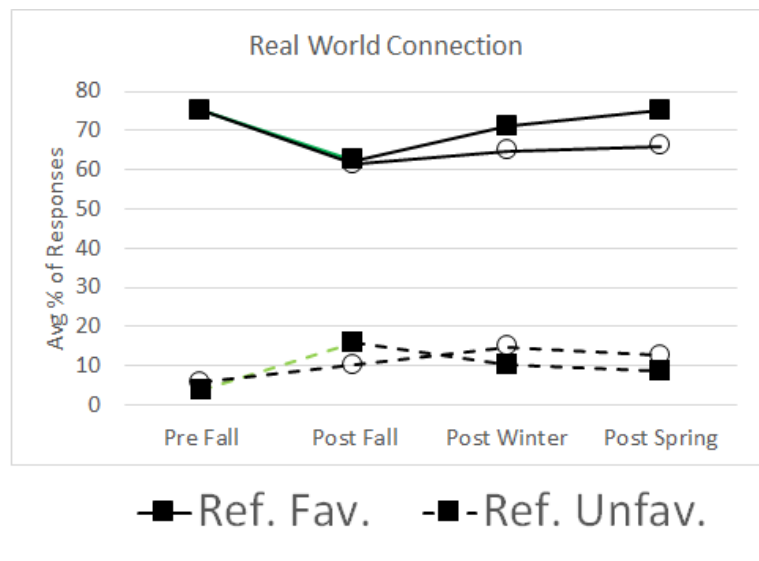

(a)

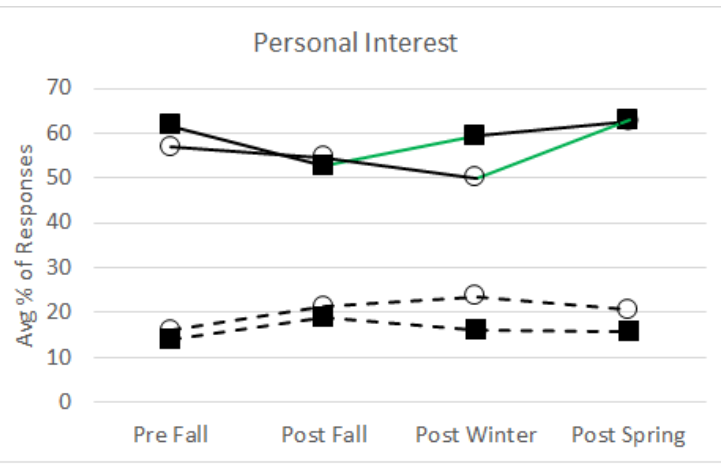

$\rightarrow$ Trad. Fav. $-\Theta-$ Trad. Unfav.

Figure 4.7: Shifts in favorable and unfavorable responses from the (a) RWC and (b) PI categories of the CLASS for the full 2015-2016 school year. 46 students from the reformed course (black squares) and 22 students from the traditional course (empty circles) completed all four surveys. Green lines represent significant shifts.

Both groups of students show a large decline in favorable responses in the RWC connection category at the end of fall term. That decline begins to reverse in winter term and continues to increase through spring term. By the end of spring term, students in the reformed course finally end at approximately the same value that they started at the beginning of the year. Although the students in the traditional course end the year at a lower level then where they started in the pre fall survey.

The PI category shows a similar trend. Students' favorable responses decrease after fall term. In winter term, the average percent of favorable responses for the students in the reformed group begins to increase. Once again, they finish the year at approximately the same value that they started at before fall term.

These results only represent a subsection of the total number of students in each course. However, similar trends are seen in the CLASS results for the PI and RWC categories as shown in Figs. 4.1 and 4.2. 


\subsection{Discussion}

Given the very large shift in PI and RWC connection seen after the 2014 pilot version of the reformed course (Fig. 2.28), it was expected that the 2016 form of the course would have had similar results. In fact, students in the traditional course showed greater favorable shifts in attitude. The exact reasons for this cannot are not known. However, there are a number of factors that could possibly have had an influence on the results.

Instructors A single instructor taught all six sections of the algebra-based physics course during the 2015-2016 school year. This was not true of the 2013-2014 year. That year the six sections were split among four instructors. It is possible that differences in teaching styles may have contributed to the large shifts seen in spring.

Although the structure of the 2014 and 2016 courses were almost exactly the same, there were some differences between the courses as a whole. Both instructors had a great deal of experience in teaching the traditional format of the course. However, the instructor of the 2014 course was one of the developers of the reformed material. This person therefore had more experience with the content than the instructor of the 2016 course.

Morning/Evening Effect The reformed course was offered in the evening section in 2014 and the morning section of 2016. Four years of CLASS data from the algebrabased introductory courses have shown that students in the evening section tend to

have greater expert-like shifts than students in the morning section. This is true of every fall term and most other terms since 2012. The CLASS data from each term can be found in the appendix (Sec. A). 
The reasons for this difference in CLASS shifts remain unknown. Different instructors have taught both sections over the years. Comparison of the students who enroll in the morning section to those in the evening section is limited because demographic data was not collected until the 2015-2016 school year. However, in fall of that year, the morning section had considerably poorer shifts than students in the evening section. No significant differences between these groups were found in age, gender, class standing, high school physics experience, or future career plans.

The simplest explanation could be the number of student in each section. The morning section tends to have a larger enrollment than the evening section. Sometimes twice as many students are in the morning section than in the evening section. A meta-analysis of 24 published CLASS and MPEX results found that there is a significant effect of class size on favorable shifts in attitude [96]. This may have been a factor when comparing the traditional evening course of 50 students to the reformed morning course of 100 students.

Another issue that could have been a factor is the classroom. The morning/evening effect is the most pronounced in fall term. This is true of every fall term since 2012 . Even though many of those courses have had different instructors, and therefore different structures, one factor that has been consistent each term has been the classroom. Each year the morning section has been taught in an auditorium with fixed, closely spaced seating. Two students from the interviews described how they found the room used in the morning section in fall and winter term of the 2015-2016 school year.

Maria: But it was impossible to take a test or... Our teacher, he would do his best to say 'I'm going to put this question up. Work with your neighbors.' But none of your neighbors would want to move over to these tiny seats. And your desks were half size. It's like, you were trying to balance your notebook and your book and 
your calculator and it was just impossible. It didn't allow for any type of comradery, if you had any desire to.

Alan: The lectures I attended in Spring, I think the environment was better than it was in fall and winter. The group work is a huge improvement. I don't know if it's because, I don't know if you've seen where it was in fall. It was just, it was like a movie theater basically without a screen. ... But now people actually seem to work on group work during group work time. And also, this is just the layout of the classroom now, TAs and teachers can walk between rows much more easily. In the old classroom, it was like a movie theater. You could only walk at the end of rows. So whenever happened at the middle was a mystery unless you wanted to walk like a crab. So you could have been doing anything there...and no one would have known. But now, you know, TAs can show up and ask you stuff and find out if you're just gaffing stuff off.

Attitudinal Shifts with a Career Focus The course reforms may be effecting students' attitude in ways that the CLASS was not designed to probe. The results in Fig. 4.6 suggest that students are not thinking of their future careers in the health sciences when they are given questions with phrases like 'everyday life' and the 'real world'. As a result, responses to the rewritten questions can differ greatly. This possibility has been noted in other research. [58]

It should be emphasized that the rewritten questions with a career focus (Table 4.3) are not validated like the original CLASS. Time and resource limitations prevented the rigorous analysis of consistency and validation that concept inventories and attitudinal surveys typically undergo. Furthermore, the six rewritten questions were posed to students in a single, stand-alone section of the surveys. The original questions in the CLASS are dispersed throughout the survey. Students may have responded differently if the same questions were interspersed in the original CLASS. 
Despite the limitations of using an untested tool, there is reason to think that students' responses would differ from the original CLASS questions. Students in the interviews who were asked, "Do you ever think about the physics you experience in everyday life?" did not cite medical examples. Instead of mentioning sound waves used in ultrasound imaging, for example, two students described diffraction of waves in the ocean.

This is not meant to suggest that reforms in content do not have an effect. The issue is that effects of course reforms that focus on students' future careers may not be clearly reflected in the PI and RWC categories of the CLASS, which puts more emphasis on everyday life.

Fall Term It is possible that course reforms could have a larger effect in fall term rather than in spring term. Analysis of the fall term data from each year the CLASS was given reveal that students in the morning section consistently show increases in unfavorable responses and decreases in favorable responses in every category. The results from the evening section show more mixed results.

The reasons for this trend is not known, however, class size could be a factor. The fall term courses had larger enrollments than those in winter or spring. A typical fall term course may have as many as 200 students in the morning section. Data from winter and spring terms tend to show more positive shifts. This could be because the class sizes are decreasing. Another possibility could be the challenge of acclimating to an introductory physics sequence. For example, students who have not completed a math course in many years can struggle with the heavy math requirement of fall term physics. This view was seen during the interviews.

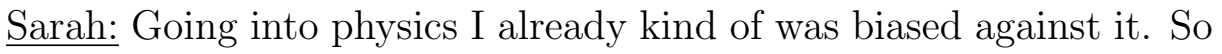


part of that was that is because it's all math. And I used to think, because I hadn't taken math in like 10 years, I thought that I was bad at math. I was out of practice at math. And so that was kind of the first hurdle.

Whatever the cause of the negative shift in fall term, it demonstrates that students' attitudes are greatly affected early in the year. Subsequent terms face the challenge of having to reverse negative attitudes, rather than building on positive attitudes that could have been fostered in fall term. It is therefore important to positively impact students' attitude toward physics early in the year. 


\section{Student Attitudes toward Physics}

At the end of fall term, students were asked the following question.

If you had the option to take either a traditional introductory physics course or an introductory physics course that focuses on the applications of physics in the health/life sciences, which would you choose?

Of the 173 students that responded to this question, $73 \%$ said they would prefer the course with the biomedical focus. The rest of the students were almost evenly split between favoring the traditional course or being unsure. This result indicates that a majority of students were in favor of course reforms at the end of fall. This chapter will explore how those attitudes evolved over the course of the year and the impact of the biomedical course reforms.

The focus of this chapter is on the survey data about students' views of their physics courses. The first section discusses how students in the reformed course responded to the biomedical focus. Students' attitudes concerning the biomedical expert interviews are detailed in the second section. The third and fourth sections detail the analysis of how students responded to the following two questions.

1. What do you view as the relationship between physics and medicine?

2. Do you think this course should be required for your major or graduate program?

Using these measures, it will be shown that students largely approved of the 
biomedical reforms because they demonstrated direct applications of physics to their professional interests. Some students had more mixed feelings about the course. This was often because they were either skeptical about the practical use of physics in biomedicine or they wanted a broader view of how physics describes the world.

Evidence will also be presented that students' attitudes seem to improve over the course of the year. This was true of students in both the reformed and traditional courses. The data suggests that course reforms might have the largest impact on students' attitude if they are adopted early in the year.

\subsection{Response to the Course}

The majority of students in the reformed course reported in the post survey that the course was an overall improvement of physics instruction (Fig. 5.1). Students' who thought the course was an improvement usually stated their opinion was due to the biomedical focus and/or the functional elements of the course like group work. Both of these features were only adopted in spring term.

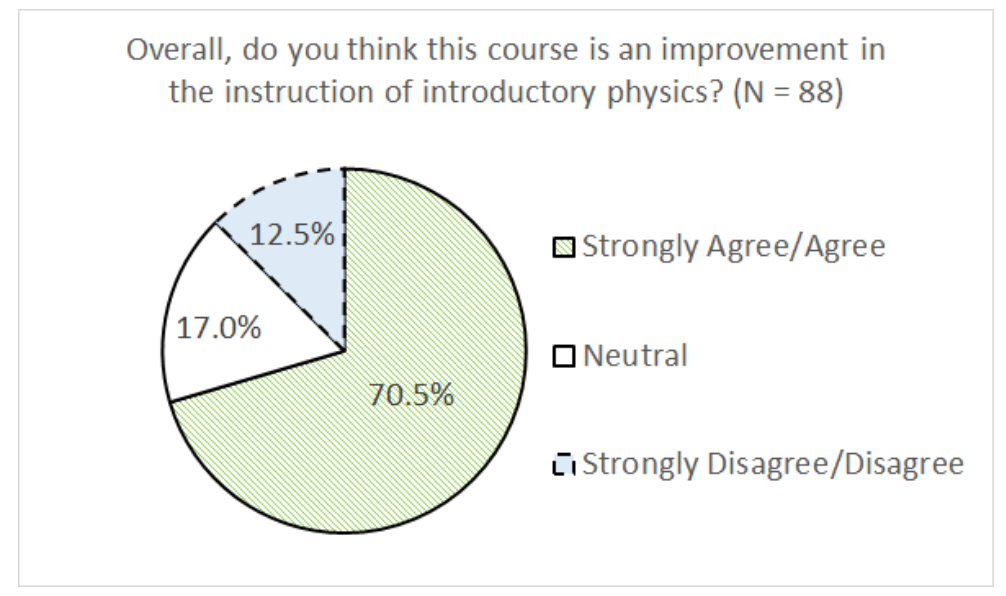

Figure 5.1: Students responses to the post-course survey indicate that the course reforms were viewed favorably by the majority of students. The prompt was a five-point Likert-style question that ranged from strongly disagree to strongly agree. The results were condensed down to three points. 
It is interesting to note that the majority of students reported that the reforms were an improvement even though they also reported spending more time on this course than previous courses (Fig. 5.2). We saw similar results from the spring 2014 pilot version of this course (Sec. 2.3.4).

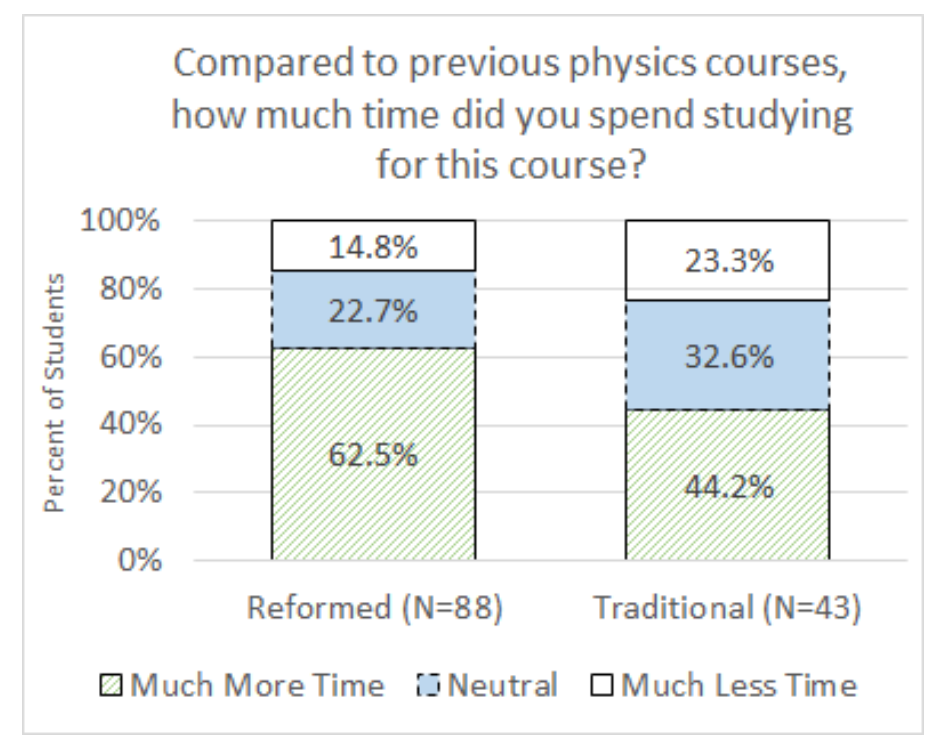

Figure 5.2: Students' ratings of how much time they spent studying as compared to previous terms. The prompt was a five-point Likert-style question that ranged from much more time to much less time. The results were condensed down to three points.

To get a better sense of whether this was true, we asked students in both the traditional and reformed courses after each term to rate how much time they spent on different course activities like homework, reading the book, or preparing for a test. Figure 5.3 shows the matched data from the post winter and post spring surveys about time spent on homework. Students were grouped according to which class they took in spring term.

The data shows that students from both groups reported spending roughly the same amount of time on homework in winter term, about 2-4 hours a week. However, in spring term, students in the reformed course reported spending more time on homework, roughly 4-6 hours a week, than students in the traditional course. The 
How much time did you spend per week outside of class on doing homework problems?

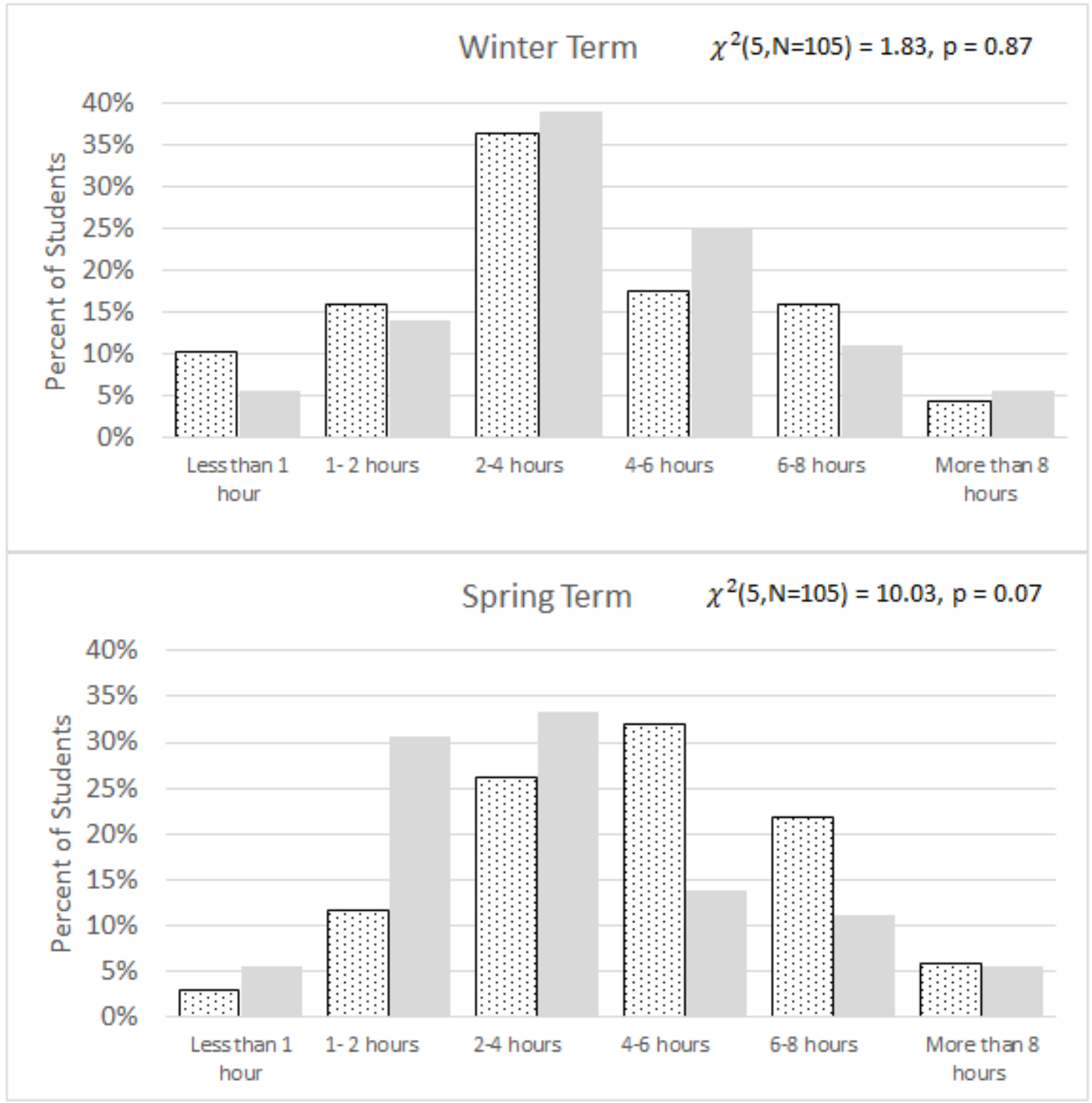

Students from the Spring Reformed Course $(\mathrm{N}=69)$

Students from the Spring Traditional Course $(\mathrm{N}=36)$

Figure 5.3: Ratings from matched students on how much time they spent on homework problems in (a) winter and (b) spring term. The prompt was a multiple choice question with the same intervals as shown in the graphs.

distribution of students in the traditional course actually show a slight shift to less time spent. Though the results show a noticeable shift, there was not a significant difference between groups. 
It is noteworthy that despite the added work, a majority of students still prefer the course reforms. Although some students stated in surveys that the added work was potentially too much to take on. In a survey, one student stated,

I prefer the structure provided with the original physics course I took in the fall and winter. I do appreciate and enjoy the biomedical context and I feel as though I learned an incredible amount, however, the biomed focus course requires much more of my time that I can't necessarily devote purely to one physics course when I am taking a full course load. I am easily spending twice as much time studying for physics this term than in previous terms, while feeling like I am performing at a lower level.

Nevertheless, the data does indicate that students prefer the biomedical focus. Some of the reasons for this are explained by responses to other survey questions. Students in the reformed course were asked at the end of spring about the impact of including biomedical applications. The majority of the students claimed that the biomedical examples improved both their enjoyment and understanding of physics concepts (Fig. 5.4).

Students described in the post spring survey what it was about the biomedical focus that impacted them. For some students, it was the connection that was made between physics and medicine.

I absolutely feel like it is an improvement for me because I am INTERESTED in the topics. I want to know why ultrasound works the way that it does. I want to understand how physics applies to my future career. This class made me see the connection and I am so much more interested now. First and second term felt like another hoop to jump through and a lot of information I would never find all that useful. Third term showed me that I was completely wrong, and now I wish I could go back and learn it in an interesting way.

The class represents a vast improvement in the instruction of introductory physics at Portland State University, and I'd recommend the course to others, regardless of their career aspirations. Physics isn't my favorite subject at all; past iterations of introductory physics have been a night- 


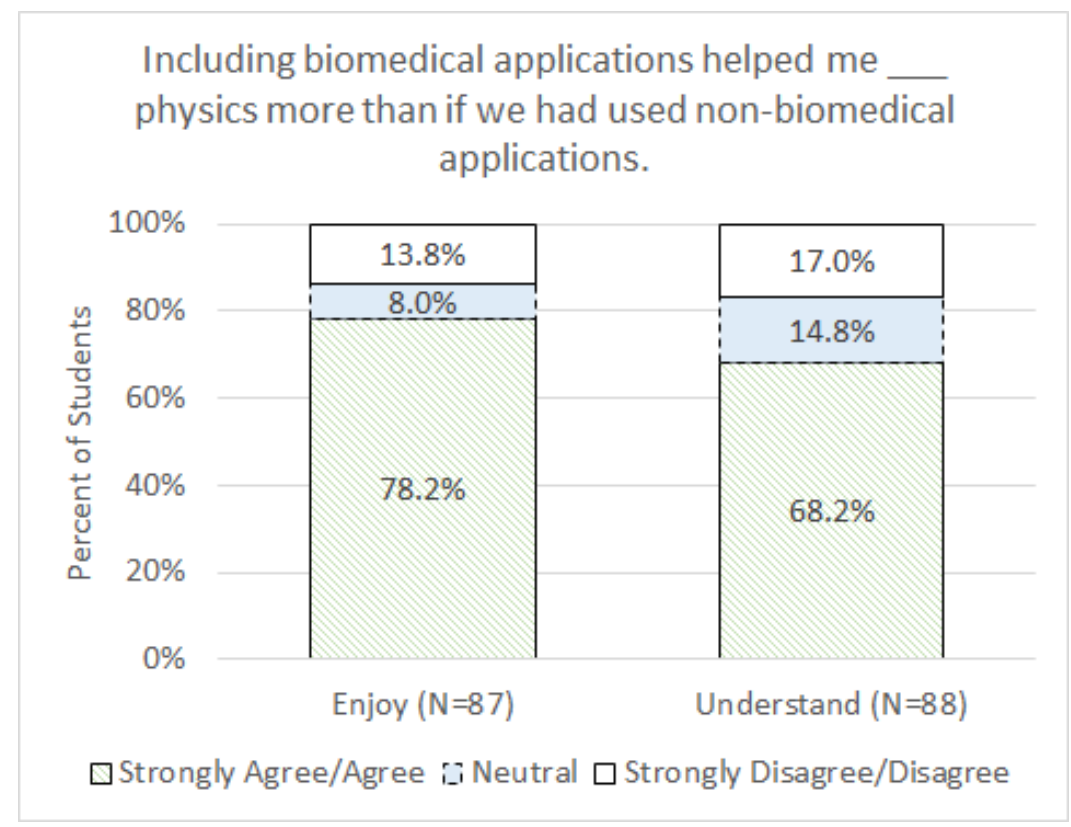

Figure 5.4: Students ratings of how the biomedical applications have impacted their enjoyment and understanding of physics. The prompt was a five-point Likert-style question that ranged from strongly disagree to strongly agree. The results were condensed down to three points.

mare for me, not to mention my GPA, but the biomedical aspect kept me interested. I actually had fun this time around, somehow. Keep the course going, I say.

The bio-medical physics has been absolute godsend. I am so appreciative that I was finally able to connect physics to medicine, and foster an interest in the course. Please make this course regularly available for other bio-medically focused students in the future.

For other students, the relevance of the material was useful, but might not have had an impact on whether they enjoyed the class more than others.

I thought applying physics concepts to biomedical tools helped make physics concepts more relevant. Whether or not I enjoyed it more because of this is hard to tell. It certainly made it easier to learn.

I think it is an improvement as it applies to health careers, but physics in general is pretty awesome! How can you improve on that, really? I enjoy physics, regardless of its application to medicine, but for students who do not enjoy it as I do, I think this course is more engaging. 
Some students thought that a more traditional focus would have been preferable. For some it seems to be a desire to see a broader view of physics. Others feel a fundamental understanding of physics is potentially more valuable.

The biomedical focus didn't make the content much more interesting considering I am fascinated with physics in general. Physics explains everything in the universe. I think that is pretty exciting.

I think it would be better for me to learn about the fundamentals of physics (in an introductory course) without learning about the application of it in medical technology. I felt like it was too much all at once. The manuscript was not helpful and I had to repeatedly use my old textbook. I liked the regular physics version better. I like it when the professor does problems on a board and explain concepts through the lens of a problem instead of a piece of technology or phenomenon.

While it is interesting to see a practical application for what we are studying I don't feel the biomedical focus adds to the material. In many ways the focus on medical principals obscures the principals behind what we are studying.

Students in both the reformed and traditional courses were asked at the end of each term whether they enjoyed discussing topics covered in each course outside of class (Fig. 5.5). The graphs show both (a) the matched data from the whole year, and (b) the matched data from the post winter and post spring surveys. Students are separated in these graphs based on which course they took in spring term. Neutral responses are not plotted for clarity. 


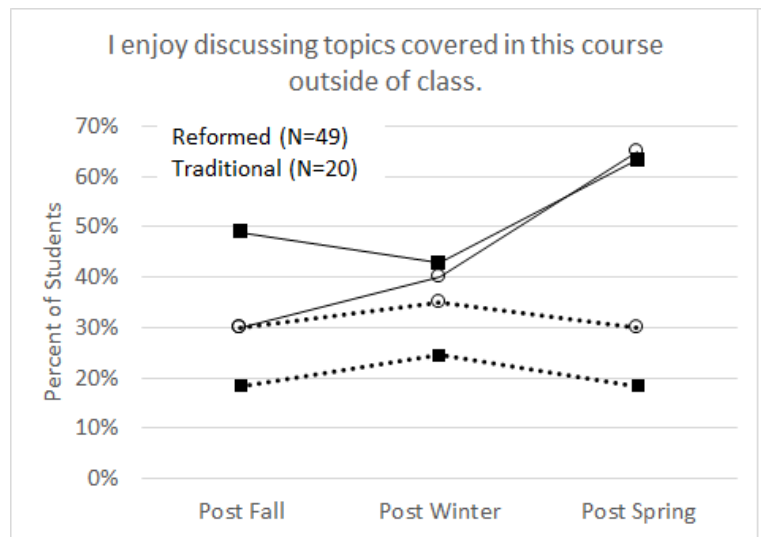

(a)

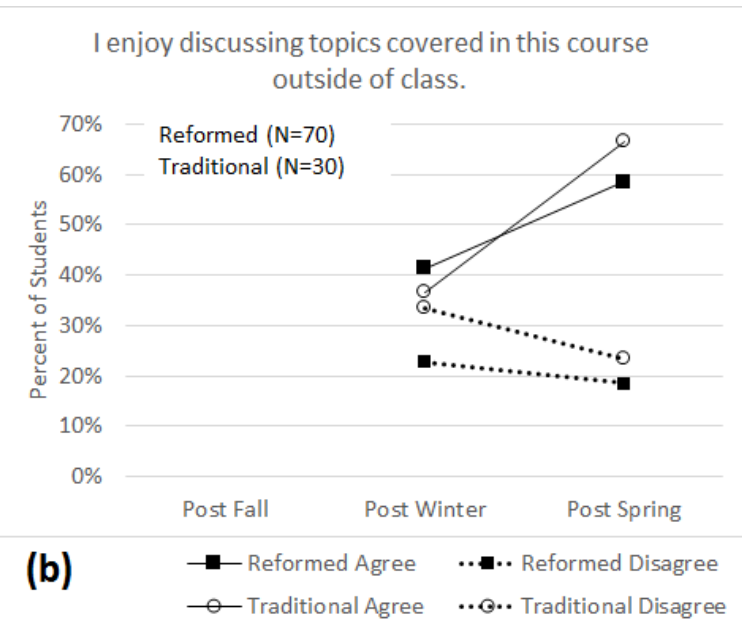

Figure 5.5: Results from the post winter and post spring survey. These results only include matched names of pre-health students who responded either (a) all three terms or (b) in winter and spring. Students are grouped by which course they took in spring term. Neutral responses are not plotted for clarity. The prompt was a five-point Likertstyle question that ranged from strongly disagree to strongly agree. The results were condensed down to three points.

Both the reformed and traditional courses show gains at the end of spring term, though the gain is larger for the traditional course. It is interesting to note that while students in the traditional course showed gains each term, the students in the reformed course show a decrease during winter. Also noteworthy is that the magnitude of the shifts in spring are much larger than the shifts in winter. It is unclear whether the gains they experience in spring are due to the course reforms, interest in the content covered in each term, or another factor such as the morning/evening effect discussed in the CLASS chapter.

Students also indicated that they think similar course reforms should be adopted for fall term (Fig. 5.6). Both the traditional and reformed courses agreed that fall should be reformed. However, the students in the spring reformed course, who experienced a physics course with a biomedical focus, were more likely to approve of reforms in fall term. 
Do you think the FALL TERM of general physics should be altered to have a biomedical focus featuring biomedical professionals explaining how they use mechanics in their careers?

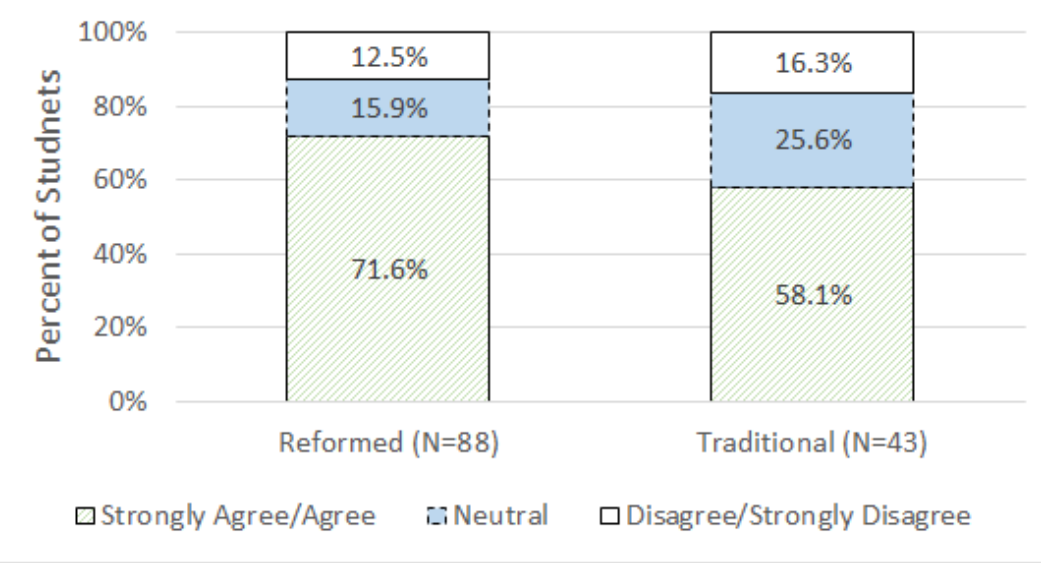

Figure 5.6: Students' ratings of whether the fall term course should be reformed to focus on biomedical applications. These results only include matched names of prehealth students. The prompt was a five-point Likert-style question that ranged from strongly disagree to strongly agree. The results were condensed down to three points.

The data in this section shows that students greatly prefer the inclusion of a biomedical focus. The remaining sections of this chapter explore in more detail what effects the reforms had on their attitudes toward physics. 


\subsection{Biomedical Expert Interviews}

Students were asked at the end of spring term what impact they think videos of biomedical experts discussing physics would have on their interest in physics and their motivation to learn (Fig. 5.7). Students in the traditional section were not shown videos of biomedical experts as part of their course work. The reformed course prominently featured multiple videos from biomedical experts. Despite this difference, the vast majority of students in both courses claimed that the videos would have a positive impact.

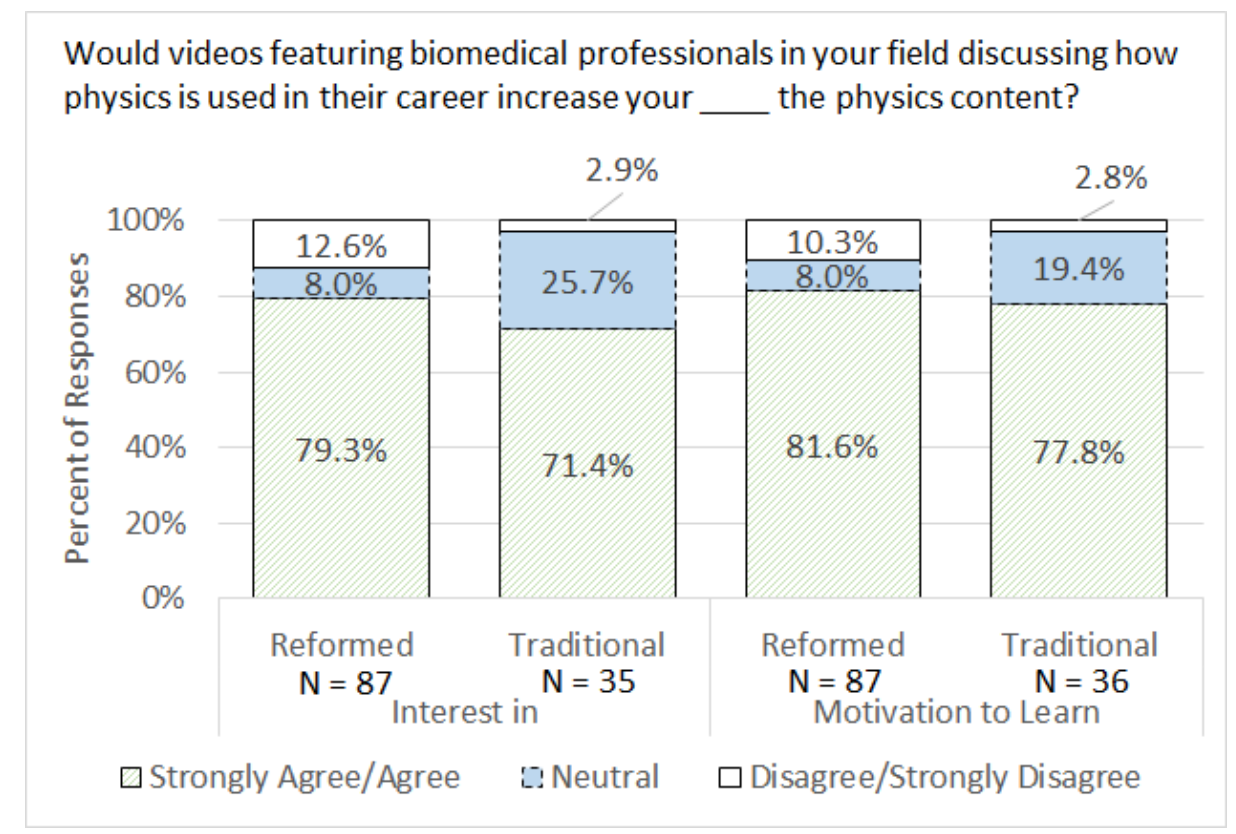

Figure 5.7: Potential impact of biomedical expert videos as reported by students. This data only includes pre-health students. The prompts were five point Likertstyle questions that ranged from strongly disagree to strongly agree. The results were condensed down to three points.

While this suggests that including videos of biomedical experts would be positively received by students, a closer analysis of the survey and interview data reveals a more nuanced view. Some students in the reformed course indicated that they appreciated 
the videos because of the links they made between physics and medicine. Students also stated that having biomedical professionals in the videos was more engaging than hearing similar content from physicists. Although for some students, videos are less engaging if they do not cover the specific specialty the student is pursuing. Furthermore, some students indicated that the usefulness of the videos was limited because the content on which they were graded had little to do with the content in the videos. This is reflected in Fig. 5.8, where a greater percentage of students rated the homework and in-class group work as useful than the videos. Some students even stated that they remained skeptical of the relevance of physics in their careers despite the interviews with biomedical professionals. These aspects will be discussed in this section.

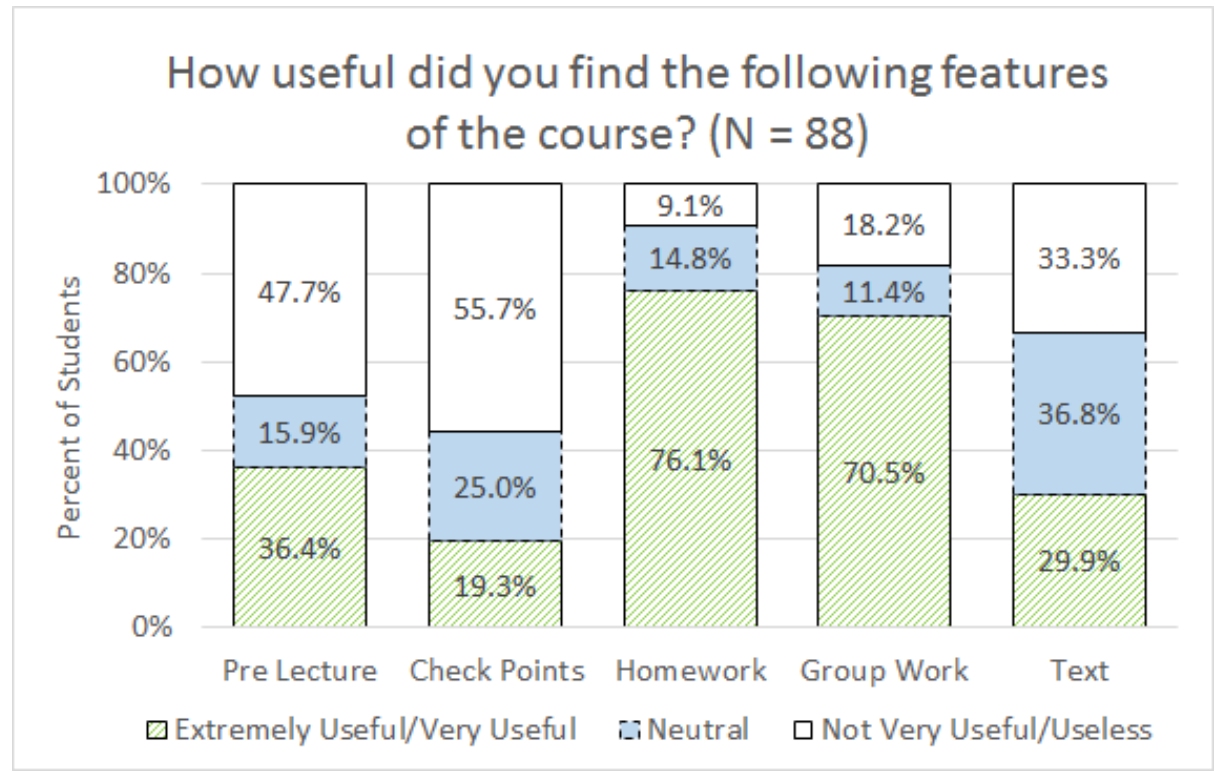

Figure 5.8: Students' ratings of the usefulness of different features of the reformed course. The prompts were five point Likert-style questions that ranged from extremely useful to useless. The results were condensed down to three points. 


\subsubsection{Connection to Medicine}

Students stated that the videos helped them appreciate the connection between physics and medicine.

I really like the way the doctors fully relate physics to what they're doing, and they often discuss connections that I don't think I would have made on my own.

It's fascinating to learn about these physics concepts through a clinical lens, as provided by the videos. I appreciate how these ideas are put into context, by hearing about how the clinicians use physics in their everyday work.

A student who participated in the interviews, who will be called Maria, described how she was surprised at the extent to which the experts use physics in their career.

Maria: And I was actually really blown away by this term. How some of our flip it models have some of these doctors from a local hospital at OHSU doing interviews and they're like really talking about these tools that they use every day and how it is so physics heavy. And it's not like something that you just learn and, "I'm a doctor now. I'm just diagnosing symptoms." It's like really a part of their everyday life. So I actually didn't realize how much it was retained. I mean, I don't know if I was ever some ultrasound technician or endoscopy doctor, if I would really think about the physics of it but they all clearly did. Cause they just did it really naturally. So yeah, it was everywhere. It was everywhere and I didn't realize it.

Students in the interviews also stated that the videos lent authenticity to the biomedical focus of the course.

Noah: I think pieces of it are definitely authentic. Especially using the videos to tie back to, you know, professionals using these ideas in their daily practice. And those professionals all obviously have a deep understanding of the concepts that we're studying. The guy they 
interviewed for ultrasound, he obviously had a deep understanding of the pieces of it. And so, yeah, I think from that aspect it feels authentic.

\subsubsection{Actual Biomedical Experts}

At the end of spring term, students in the reformed course were probed as to whether physics concepts were more engaging when presented by a biomedical expert rather than a physicist. Students were more likely to state that it was easier to engage with content presented by the biomedical expert (Fig. 5.9).

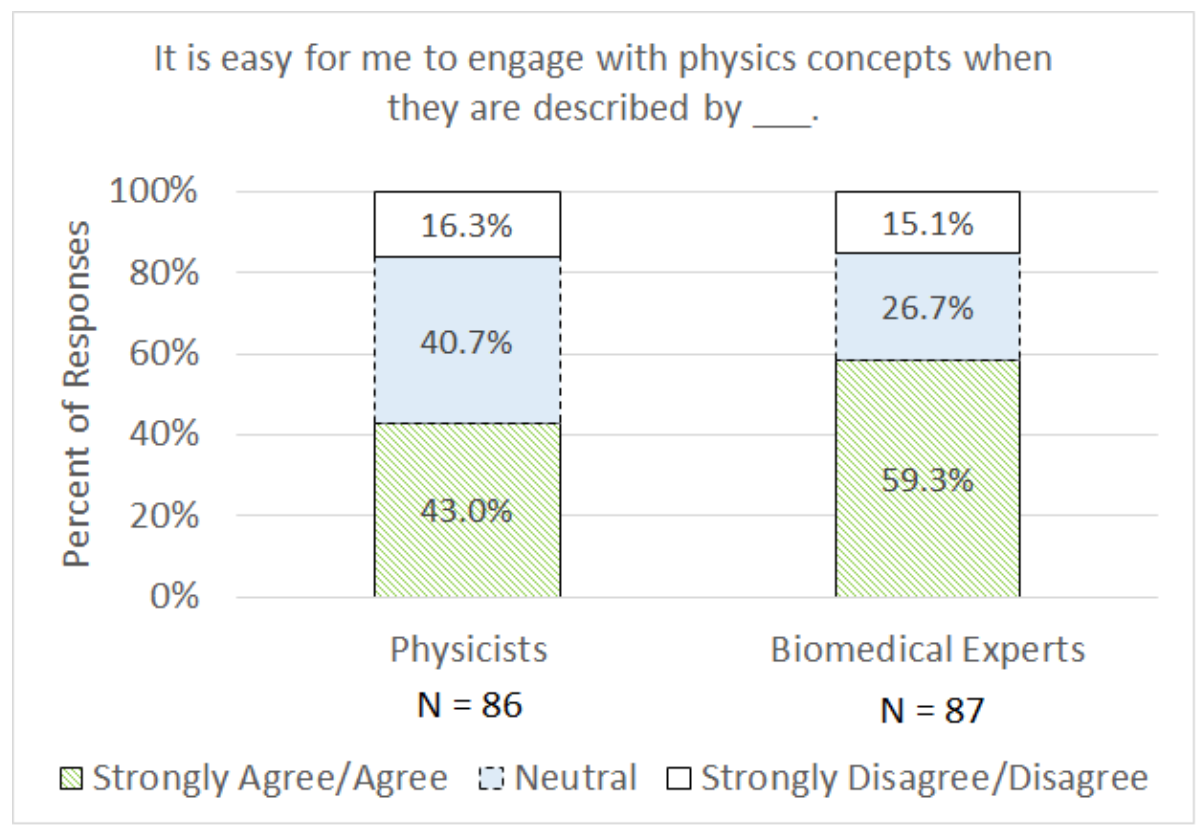

Figure 5.9: Students' ratings of how easy it is to engage with a physics concept when described by physicists versus biomedical experts. The prompt was a five-point Likertstyle question that ranged from strongly disagree to strongly agree. The results were condensed down to three points.

This feeling was echoed in the students' survey responses. One questions asked students what their favorite aspect of the videos was. Many students cited that having biomedical experts was the best part for them. "Listening to actual professionals talk about how physics is involved in their field." Another student stated, "I like hearing 
the information from actual people that study this." One student suggested that the experts demonstrate a real-world connection.

I like that the videos are directly from an actual medical professional. Any time we are able to hear how physics and physics concepts relate directly to the real world, the better we are able to learn it.

The interviews with students revealed a greater variety of attitudes toward having biomedical experts rather than physicists describing concepts. Maria stated that getting information from biomedical experts made her less skeptical about whether the concepts were of real use in the field.

Interviewer: The fact that the videos featured biomedical experts, did that impact your motivation or interest in the material?

Maria: Yeah, cause that was the thing I was saying. It easy for someone who loves physics to boast about physics. But to actually, I mean, have doctors who I really thought didn't remember the nittygritty be like 'No, I do. And I use it every day and it tells me specific things. And I do math every day.' It was more impactful to actually come straight from the horse's mouth.

Interviewer: The fact that it was actual doctors like talking about how they still use these things. That was the part of the thing that made them useful?

Maria: It was. Because if it was a physicist doing it, I wouldn't be sure that it's like not theoretical, because they're not the ones applying it. So it was nice to actually have the person who's doing the application of the theory.

Another student, named Allison seemed to agree with Maria about the credibility the experts lend to the material.

Interviewer: You mentioned that the videos that those possibly were helpful in setting the authenticity and things like that. Do you think that you'd be equally motivated, that that would help just as well if it was taught by a physicist? Or was it the fact that they were real doctors that helped in some way?

Allison: So my previous degree is in psychology. So I kind of just 
bounced into that. I feel like that there's a big part of us that does really appreciate the authenticity of a doctor telling us. Somebody who uses those tools and isn't just like, "I know how they work." But it's like, "No, I work with them every single day. This is my life." You know there's definitely something different about it.

In another interview, Hailey said that having experts was beneficial not because of added credibility. Rather it was the added enthusiasm they bring when discussing medical topics.

Interviewer: So the videos with the biomedical experts. You said that some of them were better than others.

Hailey: Yes.

Interviewer: Do you think that had any impact on your motivation for the material?

Hailey: No, I mean I think overall it was nice to see everybody. I mean whether or not I was interested in the material, everybody who was interviewed was very passionate about whatever it is that they did. And I feel that makes, to me that makes information more sticky. When you're trying to teach me something, if you're not like living, eating, breathing that subject then I, I mean I can tell and it's just 'blah'. And then I'm on Facebook or something. Interviewer: Alright gothcha. Do you think that the fact that they were actual real doctors made any kind of real difference? Or just the fact they're maybe naturally passionate about it was the thing for you?

Hailey: Yeah, naturally passionate about it. That they work in the field. They're not administration that doesn't necessarily work down in the trenches. You know what I mean? Like kind of hearing it from the horse's mouth was cool. Yeah.

Leah says what makes the videos engaging is being able to picture herself in the position of the expert. The interviewer asked her whether hearing the content from a physicist would be different than from a biomedical expert.

Leah: I don't think it would hold less weight. I think it would be less like exciting. Cause you're like, 'Oh, they're real doctors.' They're like celebrities, right. You're like, 'Ah, a real doctor. He's wearing 
scrubs!' In their white coats. You can imagine yourself there... It gives it a more exciting feel. You want to connect to it more than... That's probably human nature, but you're like, 'That's the thing I want.' You're going to connect to this.

Cathy points out that it's not the doctors that are necessarily engaging her. It's having videos rather than text that she found valuable.

Interviewer: So did the videos of the biomedical experts, did that have any impact on your interest in the in the content or your motivation for learning?

Cathy: Not really. They were really interesting, don't get me wrong. But it didn't impact me in a way that, I guess it's, uhm, it made like a live person, a live doctor person tells you about it versus just reading in a spring manuscript.

Interviewer: So the fact that it was an actual biomechanical expert added to some of the interest? The fact that that was a real, like scrubbed up doctor and not just a person?

Cathy: Yeah, not necessarily that was a doctor by any means. Just somebody that knew what they were talking about. It was just different and I guess better to have a visual of like an actual film versus just reading in a textbook.

\subsubsection{Field-Specific Videos}

Students in the interviews sometimes indicated that while they may or may not have found the videos interesting, videos from their specific field would likely have had a greater impact on them. This was certainly true of the physical therapy students in the interviews.

Noah, a physical therapy student, appreciated the videos in the spring term, but thought videos in his field would be even more interesting.

Interviewer: If you saw a physical therapist doing that sort of lesson, just as you're seeing laparoscopic surgeon doing these lessons. Would 
that video be more engaging for you than the others? Or do you think that would be about the same?

Noah: You know that's a good question. It would probably be more engaging, yeah. Because it's something that I'm specifically interested in. And you know I mean obviously there is so many different fields in medicine you know you can't necessarily touch on everybody's interest. But yeah I would probably be much more interested in it because it tied directly into my interests. Yeah.

Cathy, another physical therapy student, stated in the previous section that it was having videos rather than text that was helpful. However, she later said that would be different if doctors in her field were discussing applications of physics.

Interviewer: So as a person going into the physical therapy field, would you feel the same way if there were videos about, that were featuring physical therapists, maybe talking about some of the stuff you saw in first term?

Cathy: Yeah, probably. I'd probably be very interested in that. Interviewer: Would that be more motivating than seeing the LASIK surgeon talking about something or...?

Cathy: I think I would probably pay more attention, because they $\overline{\text { pertain }}$ to what I see my future career as and when I'm very interested in.

\subsubsection{Practical Relevance}

Some students expressed curiosity and sometimes doubt about whether the physics

they are learning in class and that is detailed in the videos is actually important to medical professionals on a regular basis.

The comments below come from students on a survey on FlipItPhysics that followed the ultrasound module. The expert interviews in this section include descriptions of how physics concepts explain artifacts seen in ultrasound imaging. Students' comments are asking how much physics knowledge is actually needed. 
One question I had after watching one of the videos about ultrasound is do sonographers need to understand all of the physics involved in the use of the equipment they are using or do they just learn how to use it?

Do the doctors in the videos learn physics more in depth to better understand their instruments, or is it mostly conceptual and straight to the point?

Just wondering two general things about the videos: were they made specifically for a class like this and to what extent are those concepts taught in medical school and used in the field?

These students appear to have accepted that physics concepts are behind what sonographers see on the monitor. However, they are not sure whether those doctors and technicians need the more in-depth analysis the students are getting in class.

One student expanded on this idea during the interviews. The student, who will be called Henry, explicitly stated that he didn't believe that physics will be practically useful. Because of this he doesn't actually watch the videos.

Henry: Currently there's like this pre lecture portion that they have

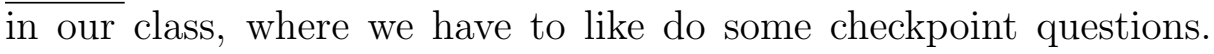
And the intention of that seems to be that they want to see that you've read the core, the material prior to coming to class. And to like spark some interest in you. You're like, "Hey look! They are talking about physics and they are in the medical community. So can you!" You know? Which from everyone I've talked to, on those videos, we're just like not even watching the videos. Or just clicking, you know, to get through it as fast as possible. And we're like, and just not really paying attention to that because it's just like... It's ridiculous. It seems kind of loaded, you know? And I'm not the only one that thinks that.

Interviewer: That the videos are what?

Henry: Like it's just, I said loaded. And the reason, what I meant by that was that... It seems that they are trying hard to make it seem like it's applicable to the medical community, when it's like, "No, it's not. I still don't believe you." 
Henry's comments seem to be based on his view that using physics means doing equations, which he said is not really used in the field. When asked whether fall term should be reformed to include more topics related to physical therapy, he said,

Henry: Yeah, I think they could definitely make it like applicable to physical therapy when it comes to load bearing and things like that. I mean, in reality in the clinics, you're not really going to be using equations like that. But it would make me more interested and it may make other students more interested in the material through that.

\subsubsection{Viewership}

Analysis of viewing habits suggests that students may have spent less time on the video interviews over the course of the term. Log data from FlipItPhysics records the time that a student first clicks on a video as well as the time of the next answer submission. This time span $(t)$ was compared to the duration $(d)$ of the video and students were separated into four groups: viewer, viewers (other), walk away, and non-access. Viewers submit an answer after $75 \%$ of the video has played. Students that submit an answer before $75 \%$ of the video has played were categorized as other. The walk-away category is for students that do not submit an answer until ten times the duration of the video has passed. Non-access describes students that did not open the video using their account. This analysis method was a modified version of the one used by the developers of the FlipItPhysics platform [114]. The criteria for each category is listed in Table 5.1. 
Table 5.1: Category definitions of student viewership of the biomedical expert interviews, where $d$ is the duration of the video and $t$ is the time from when a student first opens the video and the next submission to a question.

\begin{tabular}{l|c} 
Category & Criteria \\
\hline \hline viewer & $0.75 d<t<10 d$ \\
other & $t<0.75 d$ \\
walk away & $t>10 d$ \\
non-access & No Data
\end{tabular}

It should be emphasized that this method of analyzing viewership leaves some room for misinterpretation. The log data only records when students first open a video. Subsequent visits to the videos are not logged. It is possible that viewership is higher than Fig. 5.10 suggests, because students could have viewed the entire video on subsequent visits. Furthermore, some students may have simultaneously viewed the videos and answered questions in two browsers. Students that followed this procedure may well have been categorized as other.

The greatest variation is in the data is in the percentage of students that fit the viewers and other categories. The rate of students that fall into the walk away and non-access categories are fairly consistent throughout the term.

Figure 5.10 shows the viewing habits for each video. There is a noticeable shift away from the 'viewer' category to the 'other' category. A similar decrease in viewership during the course of the term was observed by the creators of the original FlipItPhysics content [114]. The researchers did not identify a cause for the shift other than to propose that the students had less time to use the videos by the end of term. One possible explanation of our data is that students are spending less time on the videos over the course of the term. Another possible explanation is that students are changing the way they access the videos. As mentioned above, students that open a video in one screen and answer questions in another screen would likely be counted 
as other.

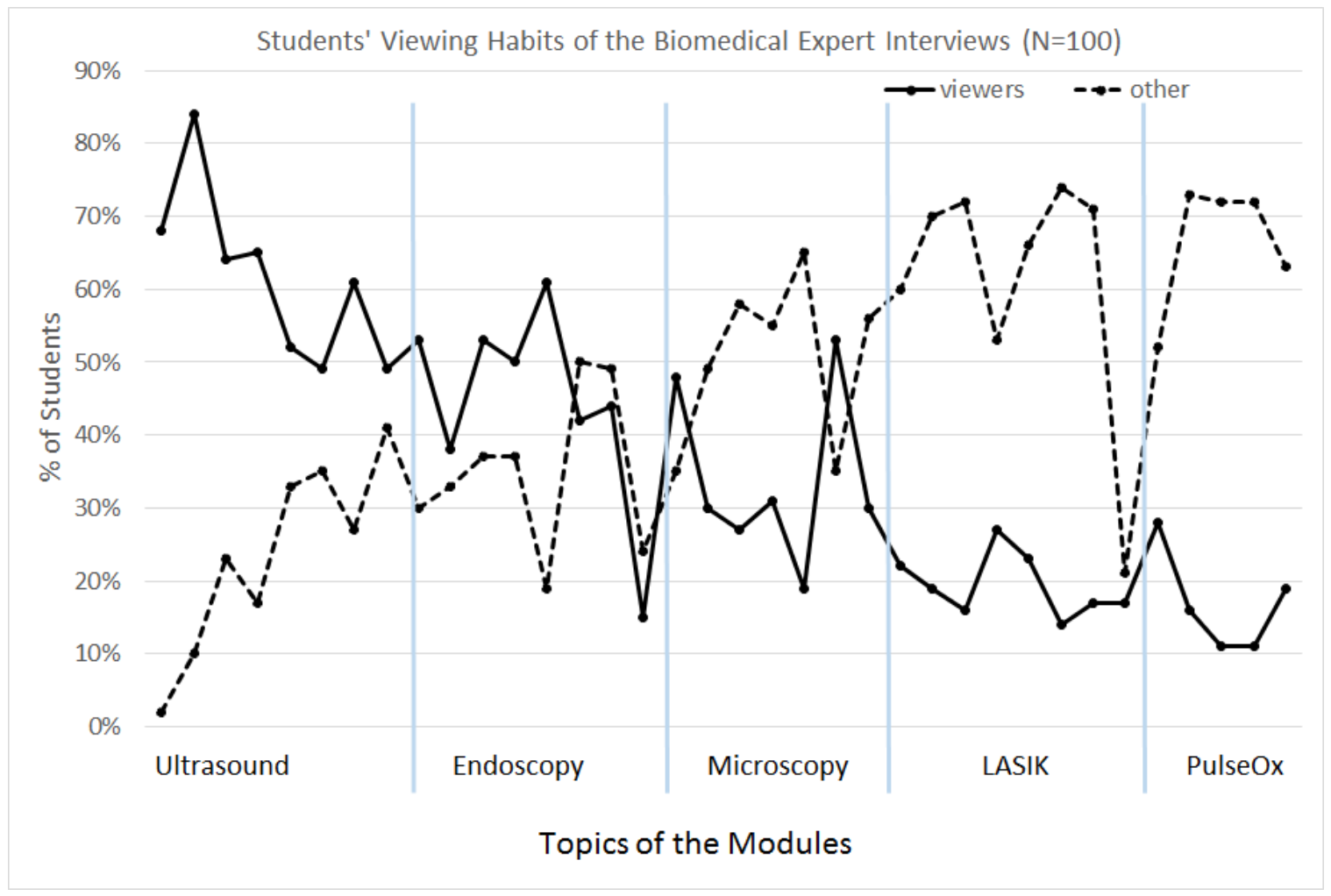

Figure 5.10: Viewership of the biomedical expert interviews. Each point represents a different video in a given module. Viewership is based on logged click traffic. Viewers are defined as student who do not click on another link for at least $\mathbf{7 5 \%}$ of the duration of the video but no longer than ten times the video duration. Others click another link before $\mathbf{7 5} \%$ of the video has played.

Despite the potential limits of the viewership data, statements from students in the interviews suggest that decreased viewership was an issue in the course. In a previous section, Henry stated the he and other students weren't watching the videos and "just clicking to get through it as fast as possible." Another student described a method to skim the videos.

Ryan: So in the beginning I actually listened to all the prelectures. Now I cheat. I uh, have you know the video on one side and the question on the other side. And I just kind of fast forward to a little bit. And hopefully hit the point where you have just one person do it and gives you all the answers. 
Another student stated that actually watching the videos wasn't always necessary to answer the questions correctly or to get good marks on course assessments like homework or quizzes.

Emily: Some of [the videos] were interesting. And honestly like I've got a lot this term. So if I had more time and I wasn't taking the course load that I'm taking, I would probably watch them. But I haven't needed to watch them to answer the questions. So I haven't... So I don't feel like watching the videos is going to help me in the homework or the quiz. And so it's like time versus benefit, and it's not high for me.

One student echoes this feeling and makes it clear that he, and other students, didn't watch videos because they were not important for getting a good grade.

Alex: There is no exam on these things, so I feel like I want to know some of this stuff but none of it's going to be important for the exam. So I'm asking myself, "Why am I spending time on it?" ... I don't even listen to the videos. I just answer questions without really... This doesn't take that much effort. You can Google, it if you need to. And also I've got a lot of friends who will just ask me for the answers. They won't even watch the videos. Get through those as quickly as possible. 


\subsection{Relationship between Physics and Medicine}

In order to understand whether pre-health students view physics as being relevant to their future career, it is important to know how students relate physics to medicine. The question is then what relationship, if any, do students see between physics and biomedicine. This was probed in a pre/post open-ended survey question and during the interviews. The results indicate that the majority of students regard physics as being important in the development of medical technology and as a fundamental science, upon which other sciences are based. Although some students do state that a deep understanding of physics is not necessary for practicing medicine, they rarely state that there is no relationship at all.

\subsubsection{Categories}

At the beginning and end of spring term students in both cohorts were asked the open-ended question, "What is the relationship between physics and medicine?" Two reviewers identified a number of categories that represented the variety of responses (Table 5.2). Descriptions of the categories and samples from each are given below.

It should be noted that these categories were not independent of one another. While the categories are representative of the topics mentioned in the responses, individual answers were sometimes more nuanced. Many students gave responses that fit into multiple categories. 
Table 5.2: Emergent categories of the responses to the prompt, "What is the relationship between physics and medicine?"

\begin{tabular}{l} 
Category Name \\
\hline Little to no relationship \\
Positive, non-descriptive \\
Fundamental science \\
Technology \\
Physiology \\
Unsure \\
Other
\end{tabular}

Little to No Relationship Responses that were placed in the 'little to no relationship' category all indicate that the student does not see physics as important information in their preparation for a career in the health sciences. Some statements suggested this very briefly. "I don't see a lot of connection for medical staff." Another student replied, "Informational and supplementary but not imperative." Many other students gave more detailed responses in which they explicitly state physics is important to medicine, but not necessarily something medical professionals need to know. They sometimes base this opinion on conversations they have had with people in their field.

I think that physics and medicine overlap in very few things. I think the most that they overlap is in regards to the machines that we used to diagnose illness. I don't think that it is super important to know all the details behind how it works.

I understand that physics plays an important role in the development of medical technologies as well as its governing of the way particles in the body interact and how the body interacts with the world around it. However, I have yet to learn a specific practical medical application for the concepts we've learned so far.

While they are intimately interrelated, I have yet to speak with a physician who believes they use their knowledge of physics from undergrad in their day to day activities.

It is my thought that studying physics this in depth is unnecessary for 
my future career as a physician. I have been told the only area of medicine where you really use physics is radiation oncology.

Some students stated that the real benefit of physics instruction was not content knowledge, but problem solving skills. One student stated,

There are some areas of physics that highly pertain to medicine, such as the flow of fluids, the movement of enzymes, the gas laws, and I'm sure other areas. I don't think this course heavily focuses on these aspects as they relate to medicine. Over all I've always thought physics was a "weeder course" designed to filter students. I think the ability to critically think through physics problems is a valuable skill that will serve students later in life. However, I don't usually think that actual material will be pertinent.

Positive, non-descriptive This category includes very brief responses in which students claim that there is a relationship between physics and medicine, but provide little to no additional detail. For example, one student replied, "They are inextricably linked." Another student stated, "There's a lot of physics involved in medicine."

Foundational Science Responses coded in the category "fundamental science" stated that physics is the basis for other fields. In some cases, students describe how they view physics as fundamental to understanding the world. "Physics is how things work, everything." Another student stated,

It is all interconnected. Increasing your understanding of the laws that dictate how the universe behaves can only help increase our understanding of the human body because it has to follow the same laws.

Other students stated physics is fundamental to medicine specifically. "Physics provides the mechanisms behind the foundations of medicine." A physical therapy student said,

Physics is about the world we live in and our health is directly correlated 
to how we move and live in our environment. To be a great physical therapist you have to understand the powers that push and pull and that can aid or hinder healing.

Technology This category describes responses which state that medical technology relies on physical principles. Some students say this very briefly, "Medical Tools". Others give specific examples of medical technology, "Instruments that are used, such as ultrasound, endoscopy". All students though state that physics is essential for the technology used in medicine.

The technologies used in the medical field, especially for diagnostics and imaging operate on fundamental physics principles that I can learn.

The majority of advances in medicine are thanks to the understanding and implementation of physics in our equipment and physiology.

Physics is the framework behind all natural sciences. Moreover, it is utilized directly on a day to day basis in the devices and instruments that medical professionals use. To not understand the physics underlying these tools, therapies, or medicines, would diminish the point of being a medical professional in the first place.

Physiology Responses in the "physiology" category detail how physics is important to understanding different functions of the body.

Physics helps explain certain mechanical processes of the human body, which can help a physician treat a patient.

I see physics as a means of understanding biological processes such as blood flow.

After shadowing an orthopedic surgeon, it seems an understanding of physics would be useful there. I'm sure fluid dynamics can explain the movement of blood through the body - wish we had learned more about this last term. 
Unsure This category includes responses where students indicate that they do not yet know what the relationship between physics and medicine is.

I'm not really sure... its been a bit harder for me to connect physics and medicine.

One student recognized that physics plays a role, they just don't know how extensive that is.

There's some relationship in certain areas, such as ultrasound, but I am otherwise unsure about its applications for other areas in medicine.

Other Responses in the "other" category sometimes cite medical education requirements. For example, one student simply replied, "the MCAT exam". Another student stated, "A physical therapist is required to complete 3 terms of physics prior to acceptance to graduate school." Students in the "other" category also infrequently cited things like reasoning skills and problem solving.

\subsubsection{Survey Results}

At the beginning of spring term, the most commonly cited relationships were technology, physiology, and fundamental science. Only a small percentage of students from either course fell in the little to no relationship category. The results from the pre spring survey are shown in Fig. 5.11. Student in the reformed course were clearly more likely to cite technology, while the students in the traditional course were more likely to cite physiology. Since the survey was given in the first week of the term, it is entirely possible that students in the reformed course had been influenced by the

biomedical topics that had already been presented to them or by the description of the course. 


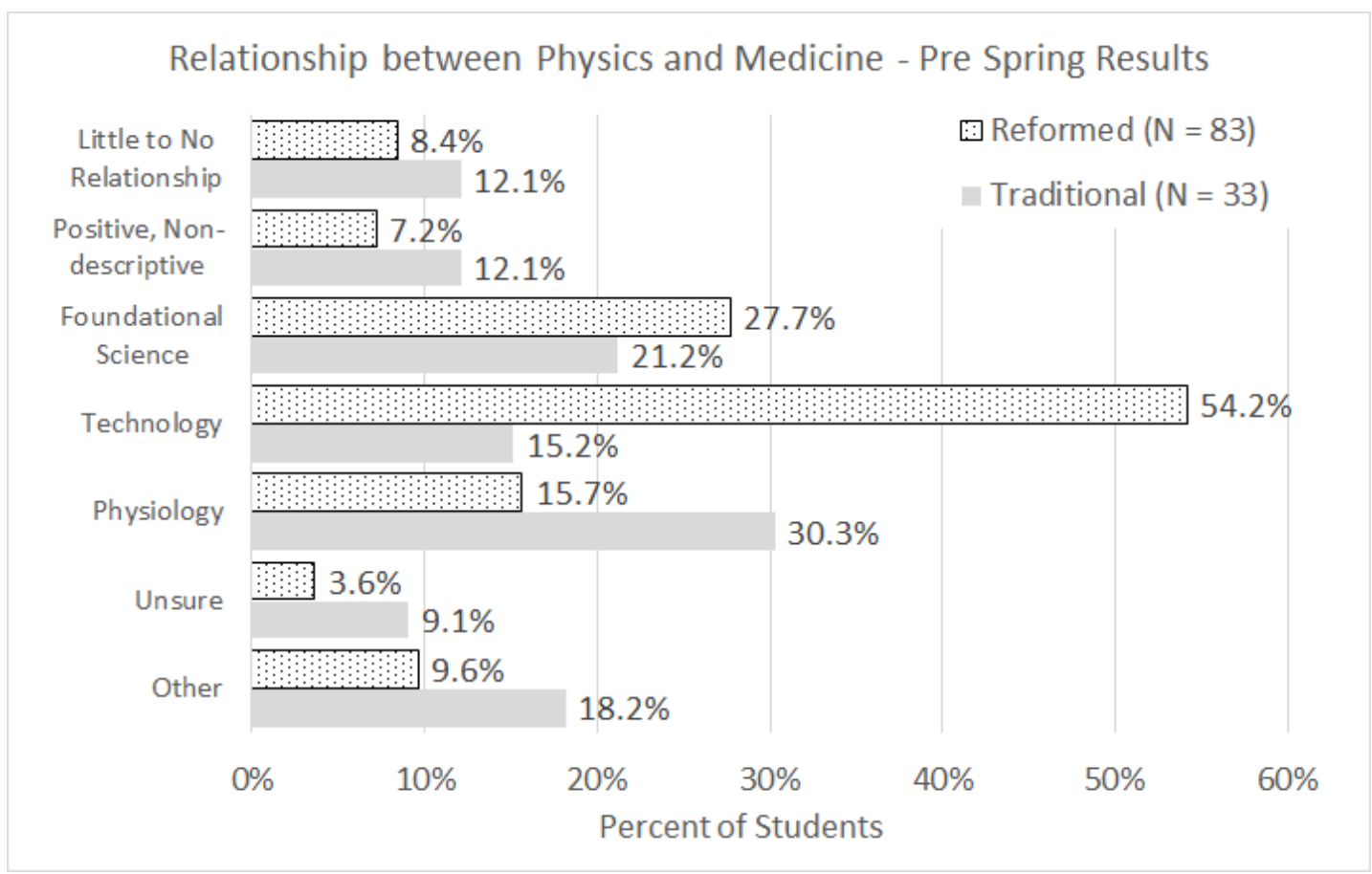

Figure 5.11: Results from the pre spring survey for students in the reformed and traditional courses. Many students cited more than one example, therefore the percentages do not sum to one hundred

Many of the students' responses cited multiple examples that fit in multiple categories (Fig. 5.12). There was not a significant relationship between the number of examples cited and the course reforms, $\chi^{2}(1, N=116)=1.12, p=0.29$.

Responses from the post survey at the end of spring term show that technology remained the dominant example given by students (Fig. 5.13). Physiology and foundational science were also commonly cited, though the percent of students from the traditional course that cited physiology decreased by approximately half. Both the traditional and reformed courses saw a large rise in the percentage of students in the positive, non-descriptive category. Once again, only a small percentage of either course fell in the little to no relationship category.

As with the pre spring survey, many students gave multiple examples. There was 


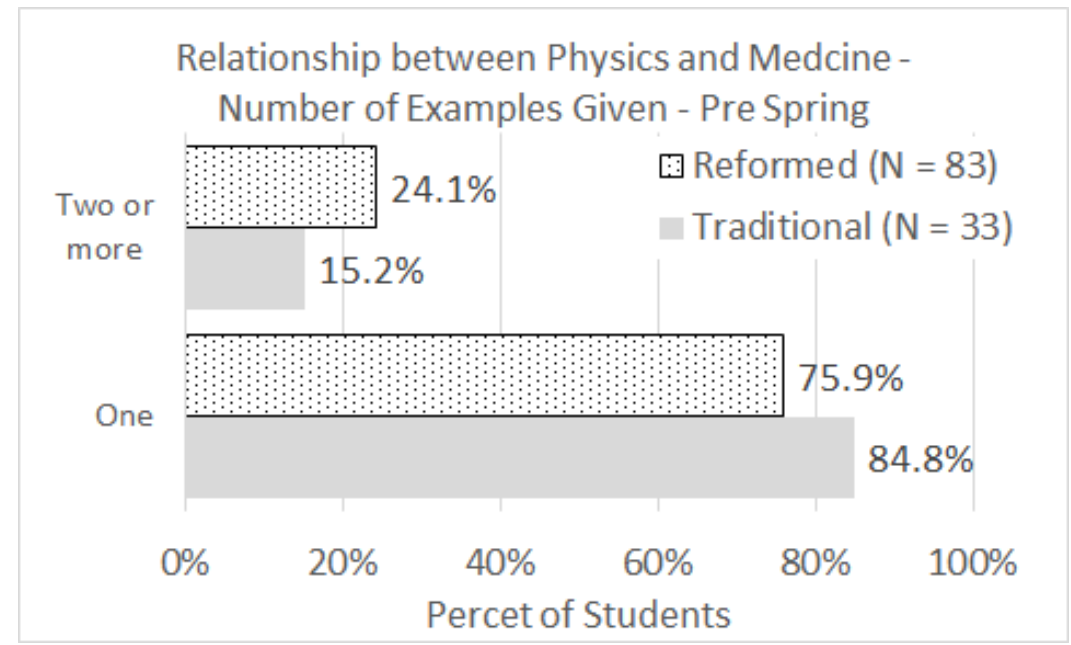

Figure 5.12: Distribution of the number of examples of the relationship between physics and medicine in the pre spring survey.

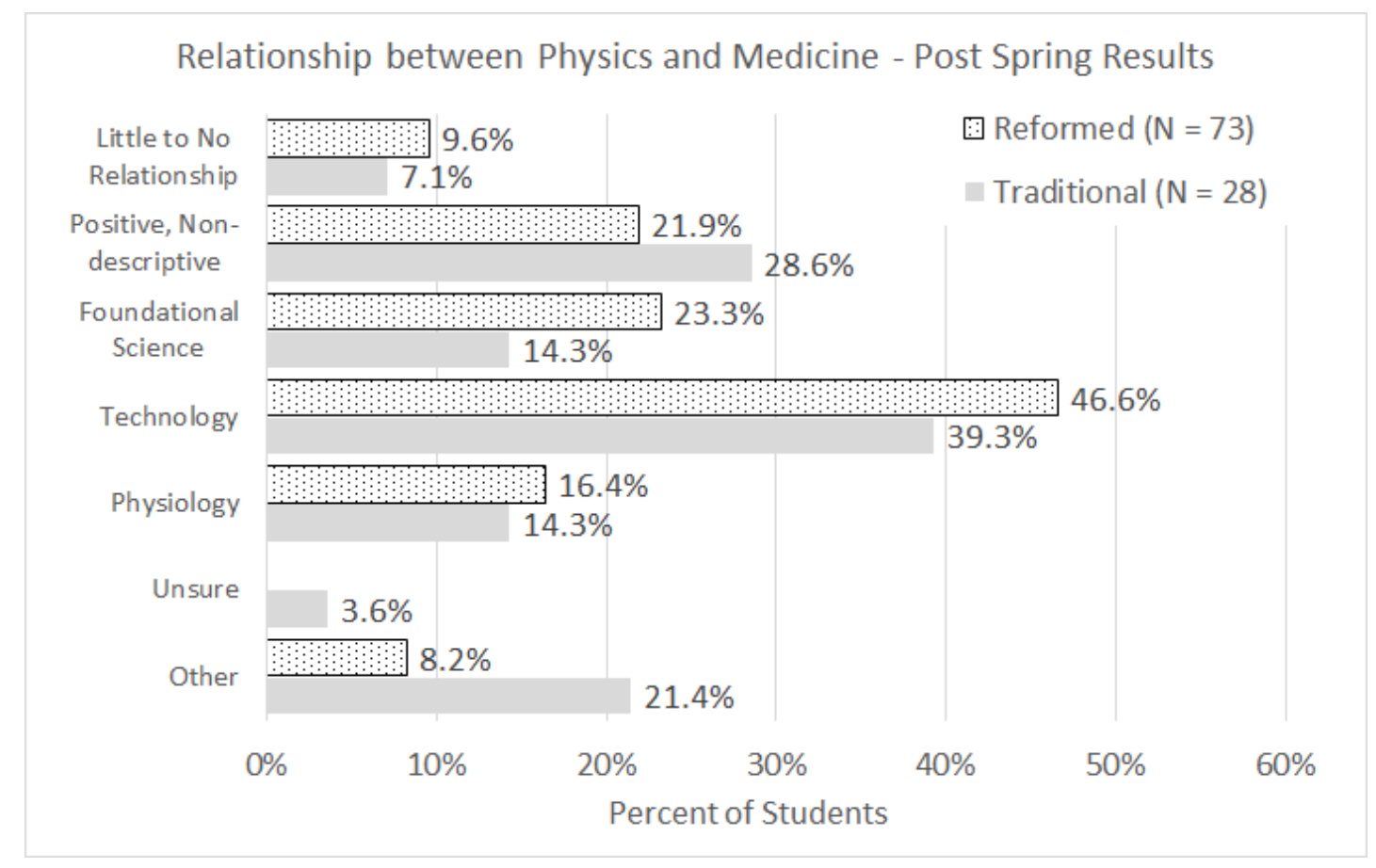

Figure 5.13: Results from the post spring survey for students in the reformed and traditional courses.

not a significant difference between the courses in the number of examples given, $\chi^{2}(1, N=101)=0.24, p=0.63$.

The results in Figs. 5.11 and 5.13 list the coding of all responses from each survey. 


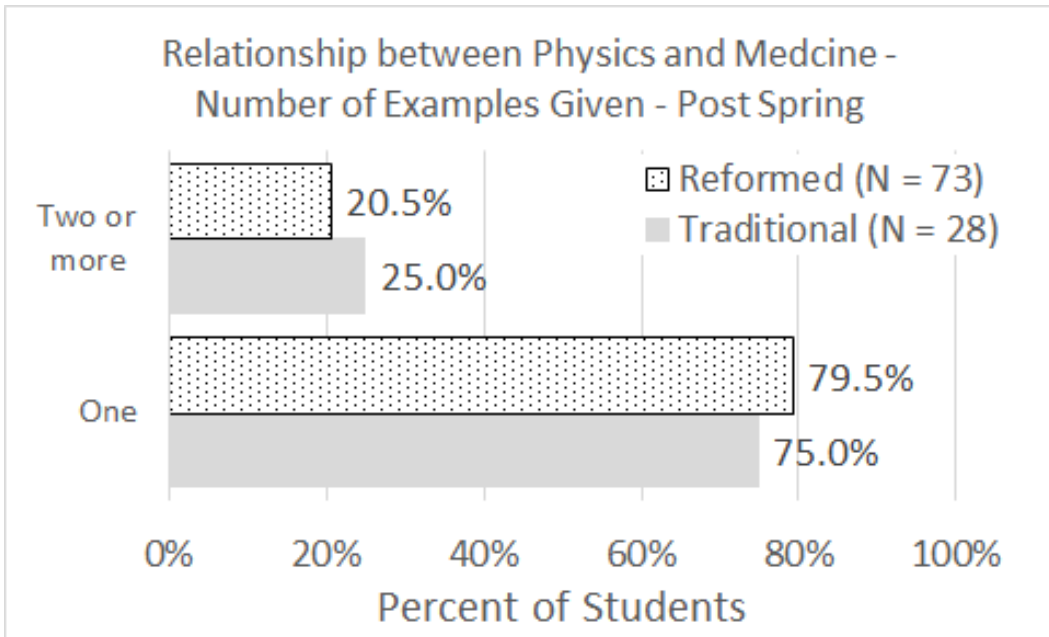

Figure 5.14: Distribution of the number of examples of the relationship between physics and medicine in the post spring survey.

The goal of the research was to explore if course reforms impacted the attitudes of pre-health students. Therefore, the results from Figs. 5.11 and 5.13 were reanalyzed to only include pre-health students that replied to the prompt in both the pre and post survey. These results are shown in Fig. 5.15.

The matched data show very similar results to those of Figs. 5.11 and 5.13. Technology is the most commonly cited example, followed by fundamental science and physiology. The largest change between pre and post surveys is the increase in technology by the student in the traditional course. Other large shifts include the increase in positive, non-descriptive by the students in the reformed course and the decrease in fundamental science by the students in the traditional course. Although both groups began the term at a similar level in this category, the students in the reformed course did not show a large decrease in the foundational responses. 


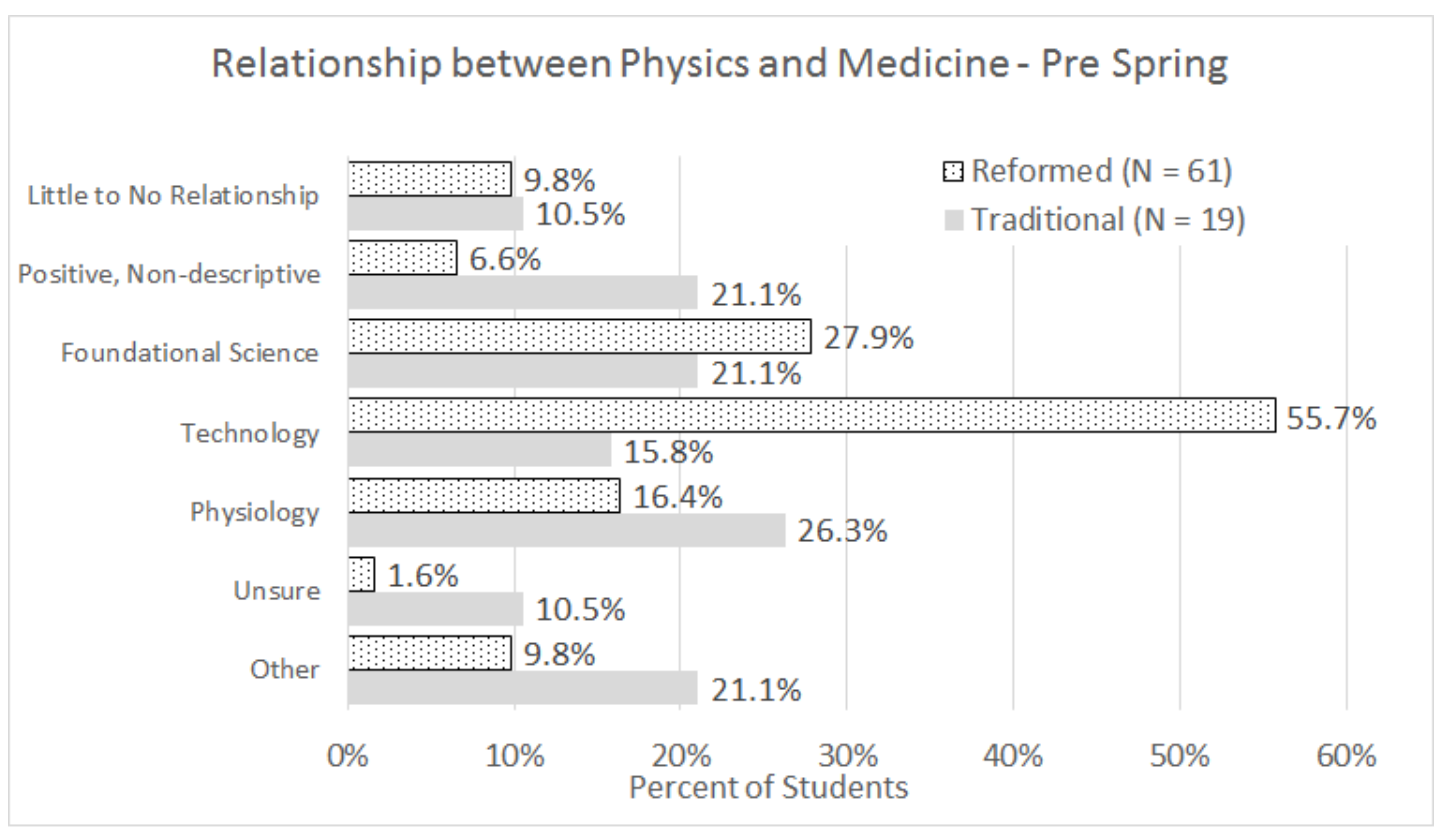

(a)

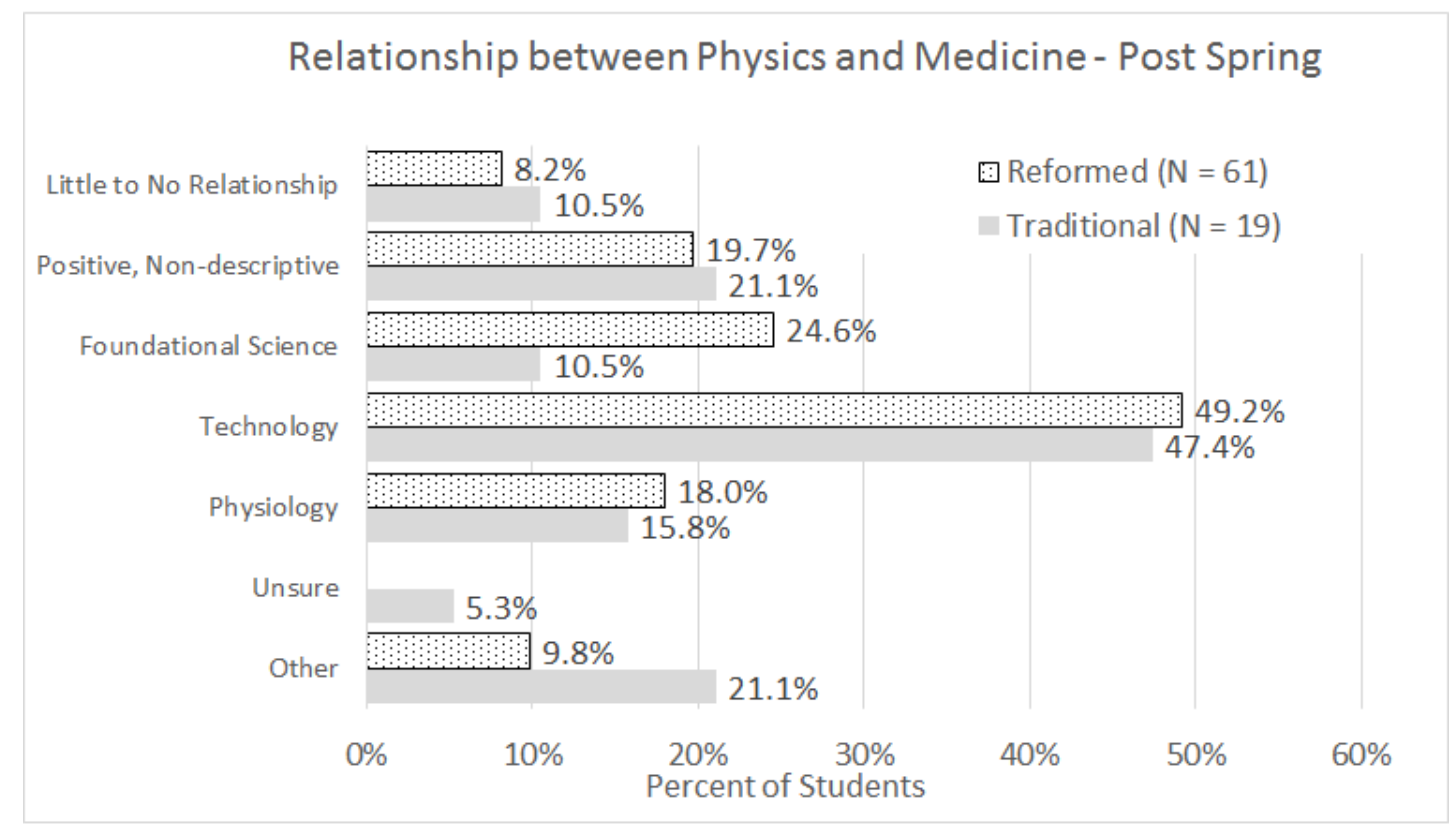

(b)

Figure 5.15: Coded responses from matched pre-health students in the reformed and traditional courses about the relationship between physics and medicine. The results are from the (a) pre spring and (b) post spring surveys. 
The matched data does not show a significant change in the number of examples given by either group (Table 5.3).

Table 5.3: Distribution of the number of examples given by students in the pre and post spring survey about the relationship between physics and medicine.

\begin{tabular}{l|ll|ll} 
& \multicolumn{2}{|l}{ Reformed } & \multicolumn{2}{l}{ Traditional } \\
\hline \hline & Pre & Post & Pre & Post \\
\hline One & 46 & 46 & 15 & 14 \\
Two or More & 15 & 15 & 4 & 5 \\
\hline
\end{tabular}

It is noteworthy that the majority of students in both the traditional and reformed courses recognized that there was a relationship between physics and medicine. Those who did not claim there was a strong relationship, still stated that one existed. Their feeling was not that it didn't exist, rather that they would not need conceptual understanding of physics in their career. As one student stated,

Many tests involve physics (ECG, MRI, ultrasound, PET scan, nuclear medicine studies...) and many therapies involve physics (radiotherapy, minimal-access surgery...) and many physiological phenomenon in the body can only be truly explained with physics. However, in the same way one doesn't need to be a mechanic to drive a car, a physician can use a tool, such as an ECG to properly diagnose and treat an illness, without needing to understand what a band pass filter is. 


\subsection{Should Physics be a Requirement?}

One indicator of whether students are impacted by course reforms must be whether they feel the course should be required. This section describes the analysis from the full year of how students rate each course from each term. The results show that students in both the reformed and traditional course were more likely to state that physics should be a requirement after spring term than after winter term. While attitudes improved in both courses, students in the reformed course were much more likely to state that physics should be required because of its relevance to medical technology. Furthermore, the improving attitudes of both courses in spring seems to fit in a more general trend of improvement over the course of the full year.

\subsubsection{Full Year Analysis}

After each term of the 2015-2016 school year, all students were asked whether they think physics courses should be required as part of their major or graduate program. This was a Likert style question, where students could select one of five options ranging from strongly disagree to strongly agree. An open-ended followup question asked them to explain their response. The responses of the Likert style question are shown in Fig. 5.16.

The first thing to note is that the data in Fig. 5.16 is meant to illustrate students' attitudes toward an individual course. Because this data does not use matched names, it does not show an individual student's change in attitude over the course of the year. Some students switch between the morning and evening sections between terms. So not all of the students in the evening section in winter term were in the evening section of fall term. Furthermore, it does not differentiate between pre-health students and 


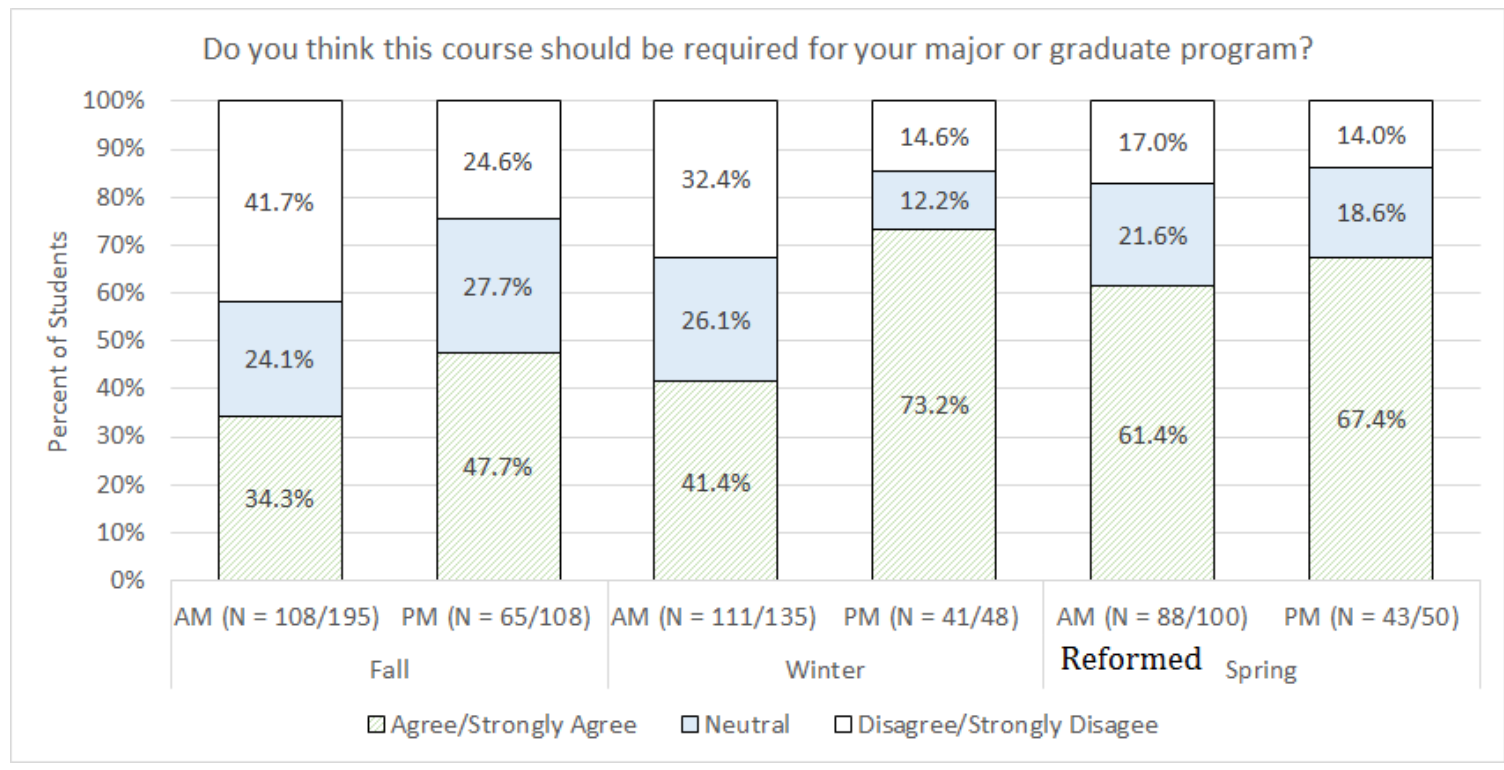

Figure 5.16: Students' ratings of whether their physics course should be required. The number of students who answered the question and the total number of students registered are given for each course. The morning and evening sections are designated $A M$ and PM respectively. The reformed course was the AM section of spring term. The prompt was a five-point Likert-style question that ranged from strongly disagree to strongly agree. The results were condensed down to three points.

students who will not pursue medicine. Matched data that only counts pre-health students will be discussed later in this section.

Students in the evening section are more likely to agree that the physics course should be required than the students in the morning section. This is true of every term, although the spring term results are almost equal. This could be further evidence of the morning/evening effect discussed in the CLASS chapter (Sec. 4.6).

Another feature of the data in Fig. 5.16 is that students in the fall term courses were less likely to think the course should be required than students in the winter or spring courses. Something similar was seen in the results of the CLASS, where spring results tended to be better than those in fall (Sec. A). 


\subsubsection{Categories}

To understand students' rational for these ratings, the open-ended follow-up question was analyzed. The prevalent reasons students cited were very similar to those given when describing the relationship between physics and medicine (Sec. 5.3). The initial coding template was therefore based on the categories used to sort students' attitudes to that question. A brief description of each category is included below.

Little to no need for physics In each term, the majority of students that disagreed that the course should be required stated that a conceptual understanding of physics is not necessary. Some students state that the real benefit is general problem solving and critical thinking.

The scope of this course seems beyond the principles and skills required for medical graduate programs. Other than practicing reasoning/problem solving skills, much of the subject matter seems unrelated to graduate program material. This class, as a pre-med requirement, seems to promote good test taking skills rather than learning and understanding the material. (It's really made me dislike physics, which is quite disappointing.)

Some students think that it's good for people in general to have this knowledge, but that the information given is too extensive.

I can not see how knowing how to apply physics equations will ever be useful as a doctor. The fundamental ideas are good for all people to know but a whole year this extensively is excessive. I think they just want to test us and see how well we can retain hard information but they shouldn't be using physics to do it.

One student expressed doubt about whether the kind of problem solving they have done in class will be useful in their career.

I don't think I'll be calculating the angular moment, kinetic energy or solving any physics problems as a pre medical student. 
Some students state that they will learn at the physical concepts they will need from other courses.

Every pharmacy student I have talked to has told me that they never again used what they learned in physics in their education/careers. Every physics concept we need to know is presented to us in the classes we use it in (e.g., biochemistry, anatomy).

The student also stated that their opinion was based on conversations with people in their field. This was also observed in the 'little to no relationship' category from the previous section.

Physics is a fundamental science Students who gave responses that fit in this category often that physics explains natural systems. "Physics shows you how the world works." Other students stated that physics connected with other sciences. "Biology, physics, and chemistry all inter-relate closely so all 3 of those majors should cross take these courses." Some students also explicitly state that physics is needed to properly understand medical topics. One student wrote,

I'm doing pre-med and I feel like the course concepts really apply to the medical and biological fields. Physics was helpful in understanding chemistry as well as physiology and the way some of the medical equipment we use works. It was also just generally really interesting, although I feel as though most pre-meds probably don't think of it that way.

Positive, non-descriptive Students in this category state that physics is important but do not specify why. "Physics is important for the field of medicine."

Physiology This category contains responses that cite process in the body that can be described or better understood through physics. "I plan to go into medicine. There are useful applications of physics in medicine for how medicines affect our 
bodies and how organ systems work with each other."

Critical Thinking This category describes statements that focus on the development of critical thinking skills developed in physics courses.

It is a challenging course and tests your ability to conceptualize things and problem solve, I find that useful for a premed student.

Although the students do not necessarily see physics as the only way to foster these skills.

I don't see how I'm ever going to use physics in Dentistry. I guess if anything it just helps me problem solve, but I'm getting enough of that in other classes.

Technology Statements classified in the technology section are very similar to those from the technology category in the relationship between physics and medicine prompt. Students state that understanding the concepts underlying medical technology will be important.

Physics is used everywhere. Technology is rapidly advancing, and I think it should be necessary to understand how these technologies work (for example, how an MRI works).

As I am preparing to enter graduate school toward a degree in Physical Therapy, I believe that many of the concepts relate to tools and equipment utilized by professionals in this field.

Physics affects medicine. The medical devices obey physical properties so it is important to understand how things work.

Unsure Students in this category given conflicted descriptions of the relevance of physics. "Some information seems related, other information doesn't." Other students do not seem to be convinced yet, but feel that the connection may become apparent 
in the future. "I'm assuming the relevance of physics in pharmacy will be clear to me as I get higher in my education path."

Additional Categories Students that supplied reasons dealing with the structure of the classes are represented in the 'course design category'. Some students simply stated that the course was a requirement for their program or that it was needed for the MCAT. They are shown in the 'requirement' category. There were also some students that stated a conceptual understanding of the physics topics would be useful. They are in the 'concepts' category.

\subsubsection{Full Year Analysis with Categories}

Whether physics should be a required course was probed with a Likert scale and an open-ended question. Not all students who answered the Likert scale question supplied a response to the open-ended question (Table 5.4). Percentages in the following graphs are taken with respect to the number of students that responded to the open-ended question. The reasons for rating whether the physics courses should be required are shown in Fig. 5.17. Data from each term are included, although the morning and evening sections were combined for improved clarity.

Table 5.4: Comparison of the number of students each term that responded to the Likert and open-ended (OE) questions about requiring physics courses. The percentages represent the fraction of students that replied to both formats. The percentage is calculated with respect to the number of students that replied to the Likert-style question.

\begin{tabular}{l|lll} 
& OE & Likert & Percentage \\
\hline \hline Fall & 133 & 167 & $79.6 \%$ \\
Winter & 119 & 139 & $85.6 \%$ \\
Spring & 110 & 129 & $85.3 \%$
\end{tabular}




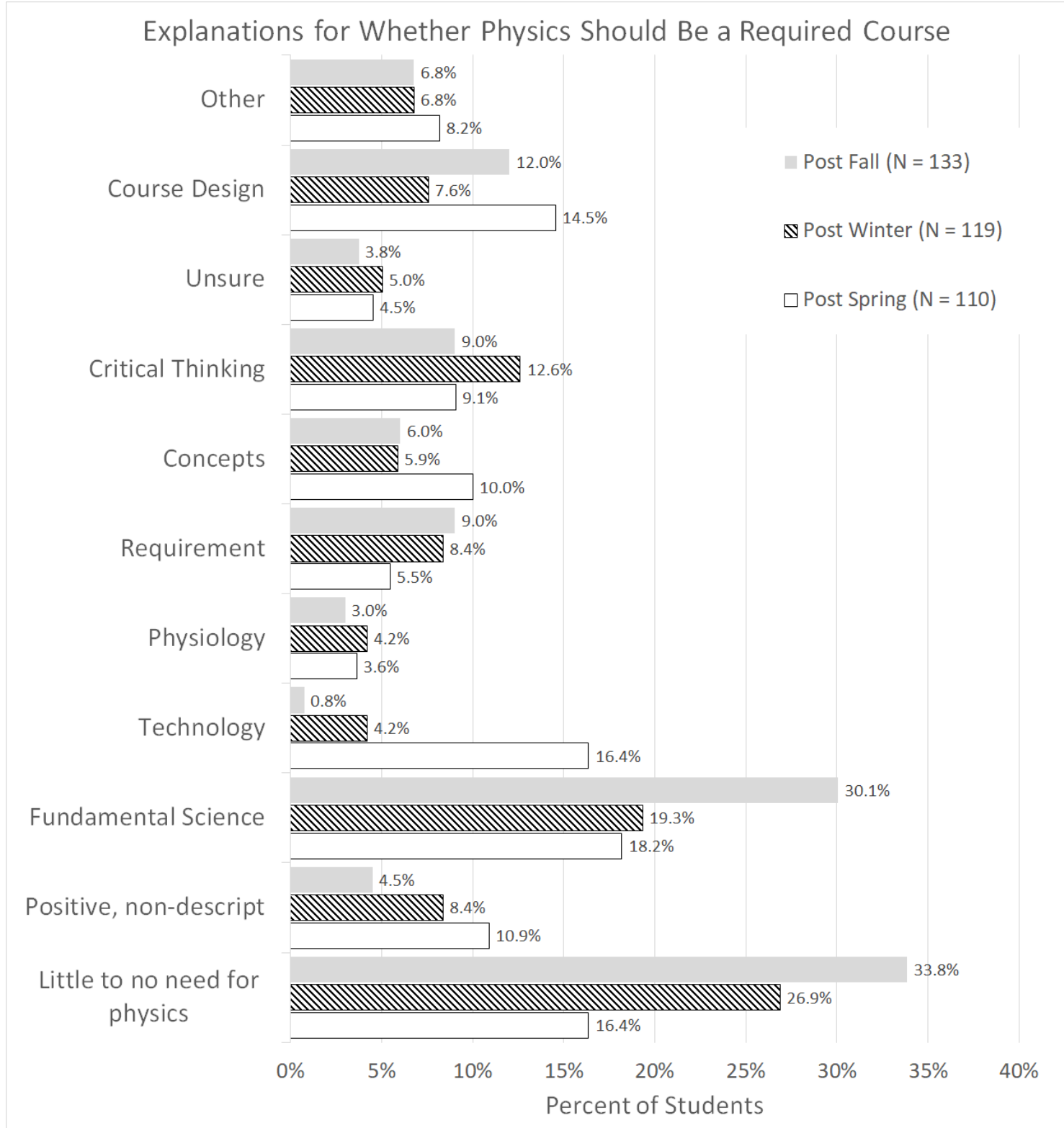

Figure 5.17: Students' explanations for their rating of whether physics should be a required course. Many students gave multiple examples and are placed in multiple categories. Therefore the percentages do not sum to one hundred.

As with the data in Fig. 5.16, the students are not separated by career path and are not matched between terms. This graph is only meant to illustrate the results of each term, not individual shifts over the year. Matched data of pre-health students will be discussed later. 
Data in Fig. 5.17 shows important trends in students' responses. Physiology was not commonly cited as justification for requiring physics courses in any term, even though it was often cited as a connection between physics and medicine (Fig. 5.11). Critical thinking was cited in all three terms in roughly the same percentage of the students. While some students did mention critical thinking or problem solving when asked about the relationship between physics and biomedicine, they represented a small percentage of the total responses.

Students in the winter term were less likely to be placed in the 'little to no need' or 'requirement' categories than those from fall term. Students in the spring term were even less likely to be place in these categories. Each term has a smaller percentage of students in this category than the one before. The opposite is true of the positive, non-descriptive category.

Following fall term, only one student from either section claimed to see how physics is beneficial for understanding medical technology. However, this student saw this benefit in a very limited sense.

Save for radiology, I can think of few medical specialties that require extensive knowledge of physical concepts. Typically, a fundamental understanding of physics, or one that might illuminate certain biological phenomena, is all that a medical doctor might need.

The technology category grew in winter term, but the larger increase was in spring. The percentage of students in the technology category in spring term was nearly quadruple the percentage in winter. This increase in the technology category in spring term was predominantly due to students in the reformed course.

Figure 5.18 shows the data for spring term separated by course type. While the percentage of students in both the reformed and traditional courses are roughly equivalent in many categories including fundamental science and critical thinking, students 
in the reformed course were twice as likely to be in the technology category. This is true even though both courses were roughly equally likely to cite technology as the relationship between physics and medicine (Fig. 5.13). Although conclusive statements cannot be made about that because the students are not matched.

Comparing the data from the Likert style question (Fig. 5.16) and the open-ended question (Fig. 5.17) suggests that student attitudes could be changing over the course of the year. Students were more likely to agree that physics should be required in winter and spring than they were in fall. Students were less likely to cite 'little to no relationship' as the year progressed. The percentage of students in the technology category increased over the year, especially for students in the reformed spring course. Although there may be a relationship, it can not be conclusively stated. So far the data presented had not been matched across terms. Matched data will be discussed next. 


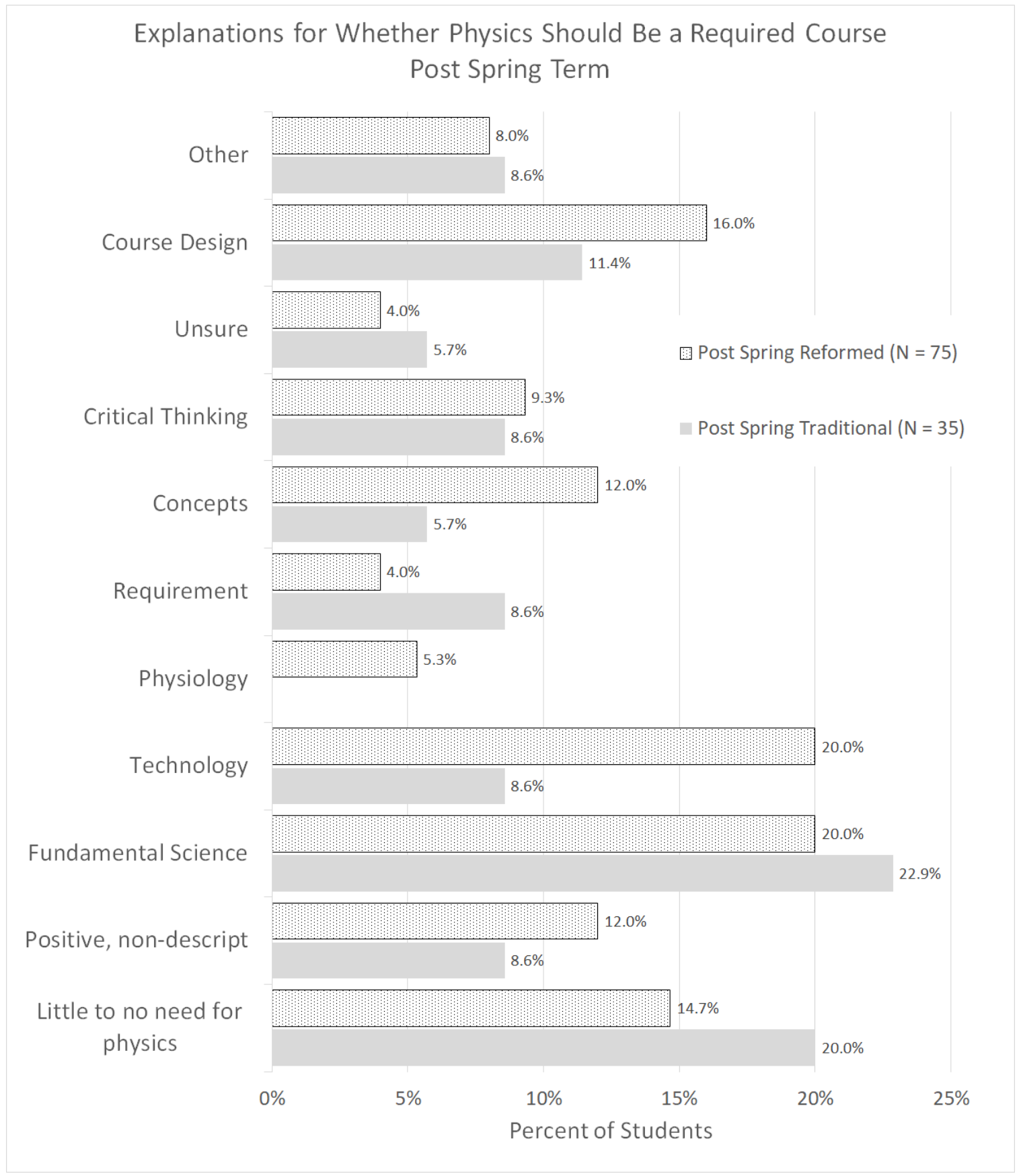

Figure 5.18: Students' explanations for their rating of whether physics should be a required course after spring term. Many students gave multiple examples and are placed in multiple categories. Therefore, the percentages do no sum to one hundred. 


\subsubsection{Matched Data from Pre-health students}

Figure 5.19 shows the matched data for the Likert style question across the course of the year for pre-health students. Because of the smaller percentage of students that replied to all three terms, data from just the winter and spring terms are also included. The students are sorted by which class they took in spring term. The neutral responses are not plotted for clarity.

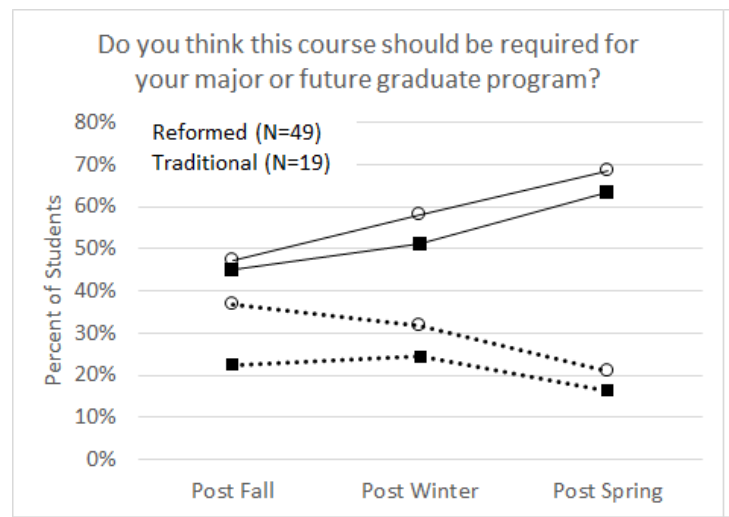

(a)

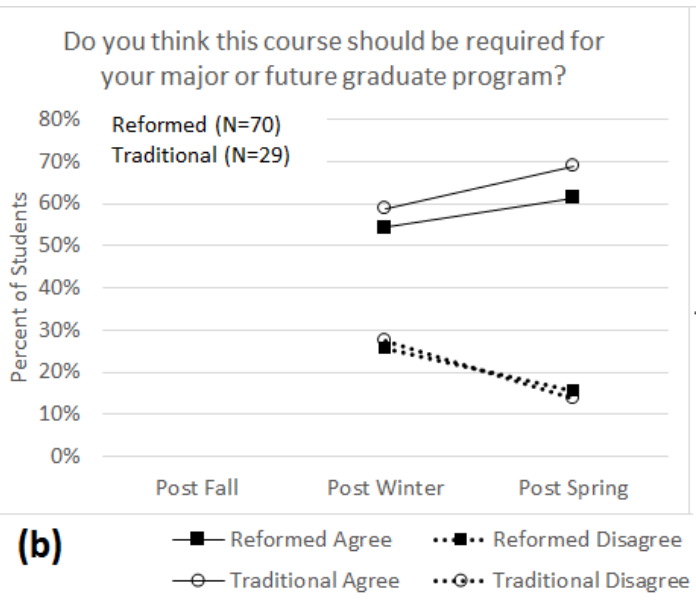

Do you think this course should be required for your major or future graduate program?

Figure 5.19: Students' ratings of whether their physics course should be required. These results only include matched names of pre-health students who responded either (a) all three terms or (b) in winter and spring. The students are sorted by which class they took in spring term. Neutral responses are not plotted for clarity.

As suggested by the unmatched data, students were more likely to agree that physics should be a required course as the year went on. This was true of students in both the reformed and traditional courses.

The reasons students gave for their rankings are shown in Fig. 5.20. This data includes matched pre-health students who responded to the post winter and post spring surveys. Note that only 61 students from the reformed course and 21 students from the traditional course gave a written explanation of their response to the Likert question. 


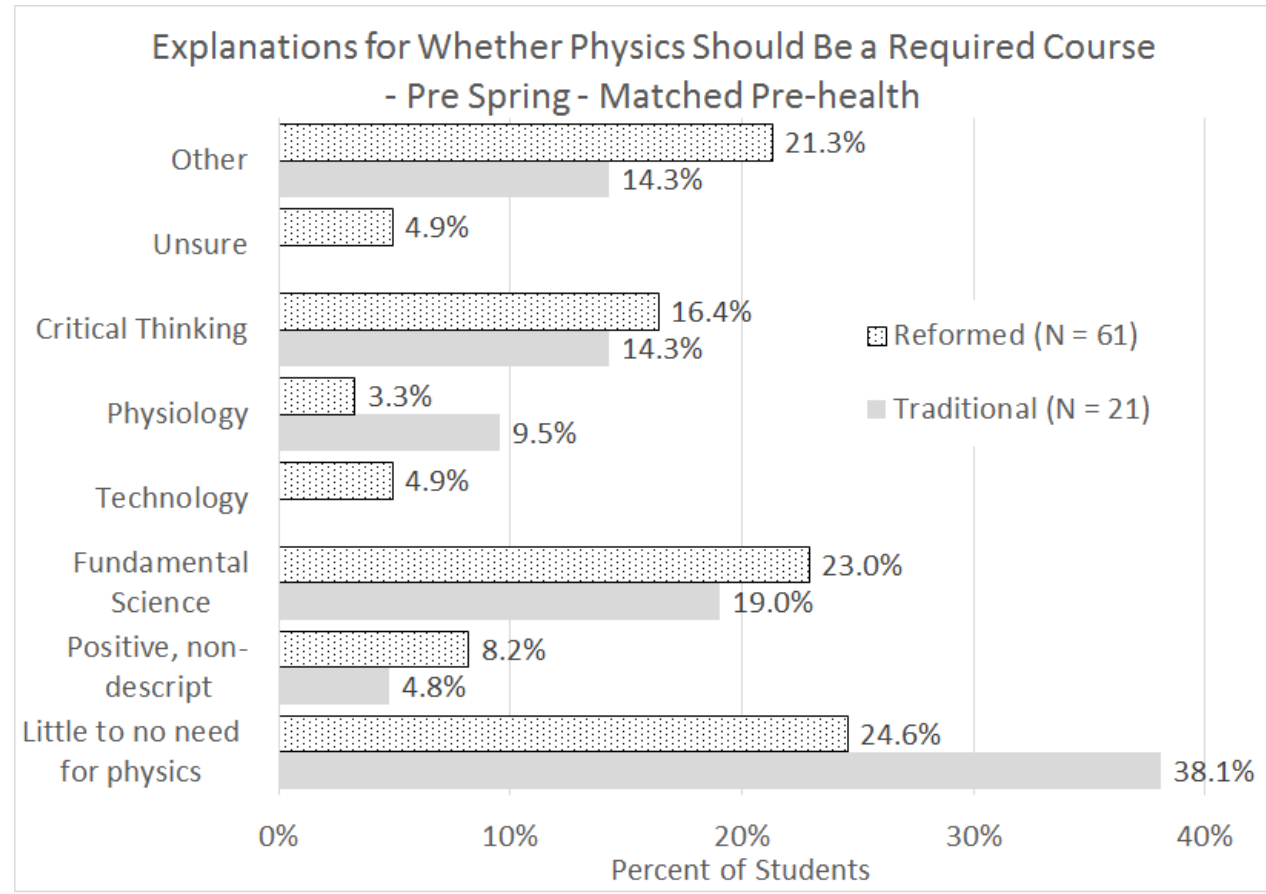

(a)

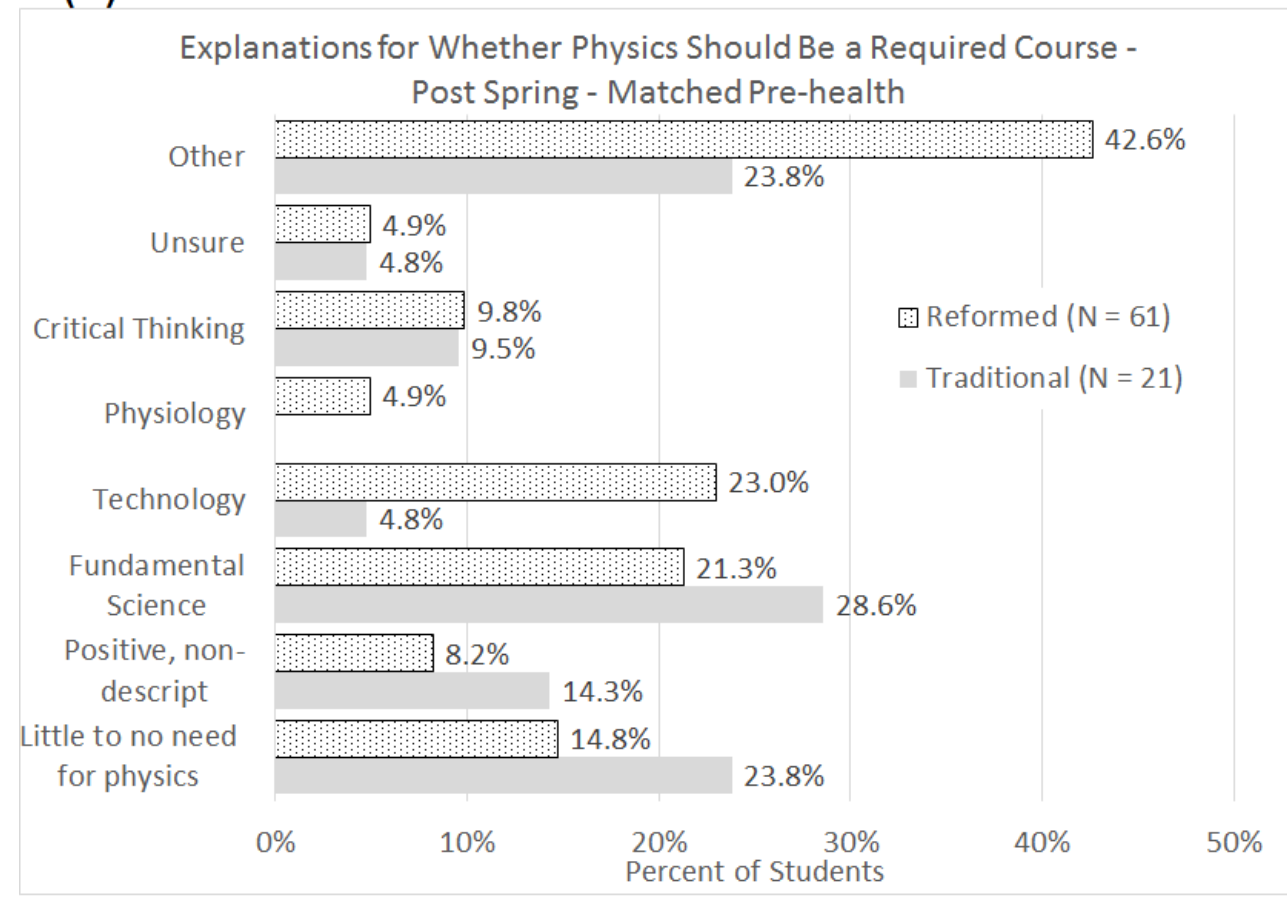

(b)

Figure 5.20: Students' explanations for their rating of whether physics should be a required course. The ratings are from (a) the post winter and (b) post spring surveys. The data includes only matched pre-health students. Some students gave multiple examples and are placed in multiple categories. Therefore the percentages do no sum to one hundred. 
For clarity the course design, concepts, and requirement categories have been collected under 'other'. Because of this the assembled 'other' category greatly increased between winter and spring for the students in the reformed course. Some of these responses discuss course format changes like clicker questions and group problem solving. These were features of the reformed course but not of the traditional.

Some of the same trends from the full year analysis can be seen in this data. Students from both courses were much less likely to be categorized in the "little to no need" group in spring term than in winter term. The percentage of students in the reformed course that cited technology at the end of spring term was over four times that of winter term (Fig. 5.17).

\subsubsection{Value of Physics Concepts and Problem Solving Skills}

In addition to questions about the relationship of physics to medicine and whether physics should be a required course, other questions were posed to students about the value of physics in their future careers.

Figures 5.22 and 5.21 show students' rating of the usefulness of the concepts and problem solving skills they learned in each course. This data only contains responses from matched pre-health students. The students are sorted by which class they took in spring term. The prompt for both questions was a five-point Likert-style question that ranged from strongly disagree to strongly agree. The results were condensed down to three points.

Both groups of students show an increase in the usefulness of problem solving skills. Although the time when the shift happened seems to have been different for each group. Students in the reformed course show a larger improvement in winter 
term, while the students in the traditional course show a larger shift in spring term.

The reformed and traditional groups show large differences at the end of fall term on the question concerning concepts, but approximately equate in winter term. Interestingly, the students who would go on to the spring reformed course seem largely unaffected by winter term. Both groups continue to improve through spring term, though the students in the traditional course had a slightly larger shift.

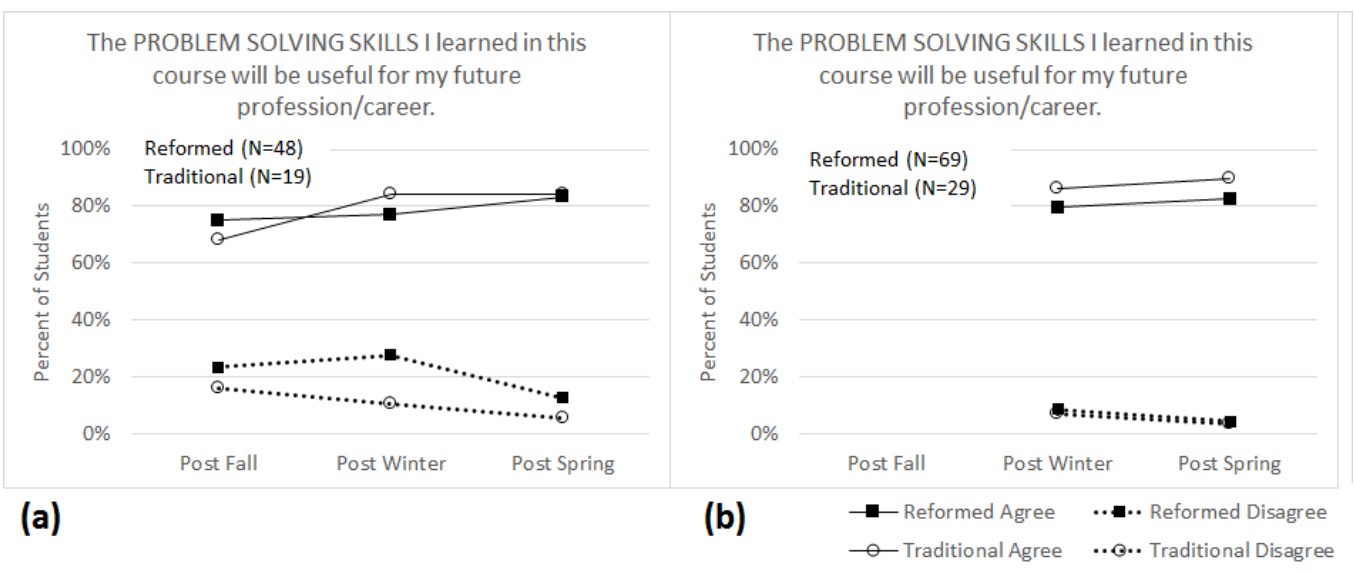

Figure 5.21: Students' ratings of whether the problem solving skills they have learned in their course will be useful in their future career. These results only include matched names of pre-health students who responded either (a) all three terms or (b) in winter and spring.

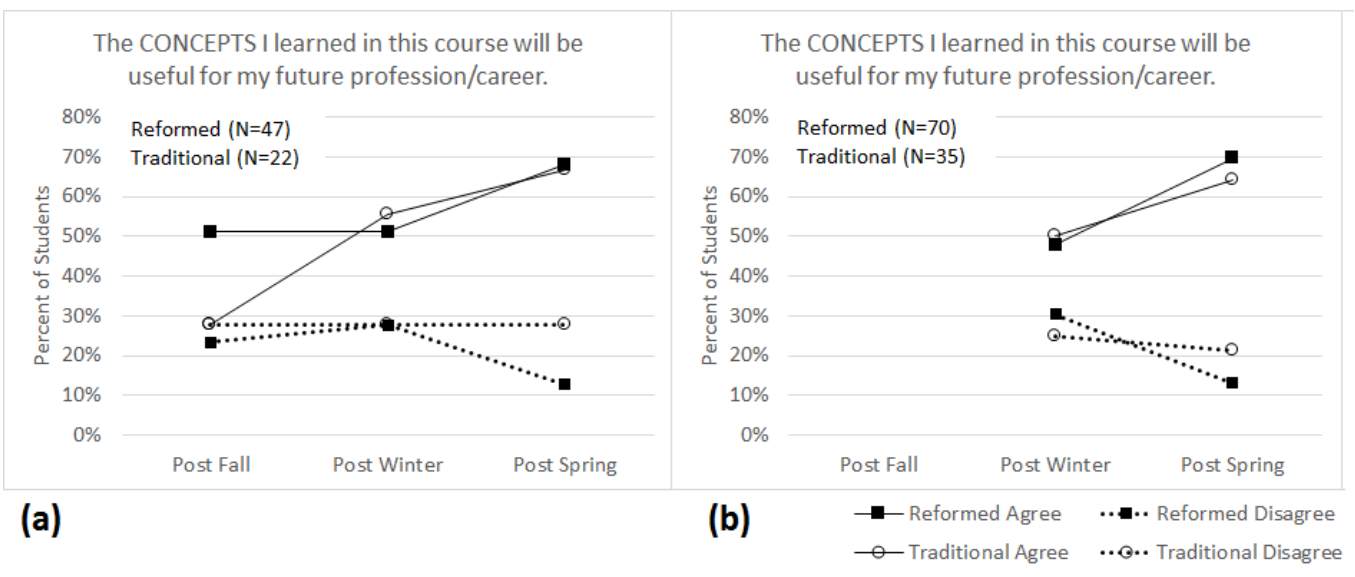

Figure 5.22: Students' ratings of whether the concepts the have learning their physics course will be useful in their future career. These results only include matched names of pre-health students who responded either (a) all three terms or (b) in winter and spring. 


\subsection{Discussion}

The analysis in this chapter shows that students' attitudes were impacted by the biomedical focus of the reformed course. Evidence was also found that attitudes improved over the course of the year regardless of biomedical reforms.

The majority of students in the reformed course indicated that they approved of the course reforms. This was true even though they also reported that the course required a greater time commitment than previous terms. Many of these students stated that the biomedical focus of the course helped them to see how physics is related to their future professions. This was reflected in their comments and the large percentage of students in the technology categories of the relationship and requirement survey questions.

There were students, though, that remained skeptical of the course reforms. Some felt that they would prefer a course with a wider scope that included medical and non-medical applications. Other students preferred the biomedical focus, but did not think that physics was going to be of practical use to them. They often acknowledged that physics was important to medicine but practitioners would not need the kind of detail learned in the course. However, it was not the goal of the course reforms to suggest that this detail was in fact directly useful, but rather to engage them through their existing interests. The fact that some people retained this belief suggests that this might not have been made clear to the students.

While the attitudes of the students in the reformed course improved over spring term, similar results were seen from students in the traditional course. Students' ratings of whether physics should be a required course rose each term for both groups. Because this question was no tasked of students at the beginning of the year, it is 
not known whether we would have seen decreases over fall term as was seen in other attitudinal questions like the real-world connection of the CLASS. Students were less likely to claim that there was little to no need for physics after every term. The percentage of students that said they enjoy discussing physics topics outside of class increased for both groups. By the end of the year, both groups were also more likely to find the concepts and problem solving skills they learned in physics to be useful for their careers. This all suggests that students' attitudes improve over the year. Course reforms meant to improve students' attitude toward physics could therefore have the largest impact in fall term.

Many of these attitudes will be discussed further in the next chapter, which deals with the student interviews. 


\section{Interview Analysis}

This section will use students' interviews to better understand some of the trends in the data, many of which were also expressed in the interviews. Some students are very favorable to the course reforms because of the application to biomedicine. Other students remain skeptical of its practical use. During the interviews, some students indicated that they would prefer a course with a wider scope than just biomedicine.

Table 6.1 lists each of the students that participated in the interviews, their planned profession, and their ratings of whether physics should be required for each term. They are sorted by their response in spring term.

Table 6.1: Students that participated in the interviews and their ratings of whether the course should be required. The students are sorted according to their rating of the spring term course. $(\mathrm{PT}=$ physical therapy, $\mathrm{PH}=$ pre-health, $\mathrm{NA}=$ no answer $)$

\begin{tabular}{l|l|l|lll} 
Name & Career & Gender & Post Fall & Post Winter & Post Spring \\
\hline \hline Maria & PH & Female & agree & agree & agree \\
Sarah & MD, PhD & Female & agree & agree & agree \\
Allison & PT & Female & agree & agree & agree \\
Cathy & PT & Female & NA & agree & agree \\
Hailey & MD & Female & neutral & agree & agree \\
Emily & MD & Female & neutral & neutral & agree \\
Leah & MD & Female & disagree & disagree & agree \\
\hline Alan & MD & Male & neutral & agree & neutral \\
Henry & PT & Male & NA & neutral & neutral \\
Noah & PT & Male & agree & NA & neutral \\
April & MD & Female & NA & disagree & neutral \\
\hline Alex & MD & Male & disagree & disagree & disagree \\
Ryan & MD & Male & neutral & disagree & disagree \\
\hline
\end{tabular}


These students show a variety of attitudes toward their physics classes. Students like Maria, Sarah, and Allison had favorable ratings all three terms. Hailey and Emily's were neutral in at least one term but positive about spring. Leah had an unfavorable view of physics courses until spring term. Alan, Henry, Noah, and April all had mixed feelings about physics courses. Alex and Ryan did not hold favorable attitudes in any term.

Figure 6.1 shows the ratings from the interviewees and those of the rest of the students in the reformed course. There was not a significant difference between these distributions, $\chi^{2}(2, N=88)=0.76, p=0.68$.

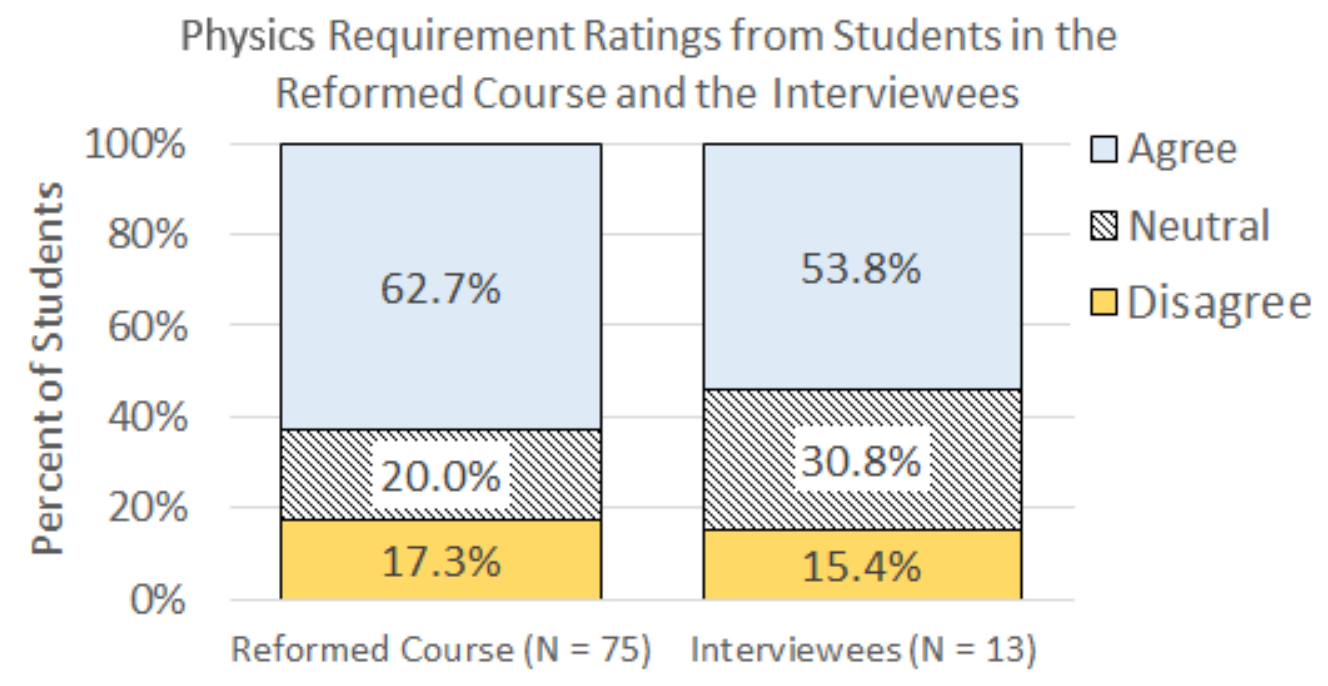

Figure 6.1: Students' ratings of whether their physics course should be required from students in the reformed course and students that participated in the interviews.

The rest of this chapter is sectioned by the ratings students provided after the reformed course in spring term: agree, neutral, and disagree. Each students' survey responses will be addressed first. The attitudes in those responses will then be expanded upon by comparing them to the student's interview. 


\subsection{Should spring term be required? - Students that agreed}

All the people who agreed that physics should be required stated that spring helped them see the relevance of physics in medicine. For many of them, the fall and winter ratings were based on the feeling that the importance of physics was as a fundamental science. Some students claimed that either they saw no relationship between physics and biomedicine until spring term, or that they only saw vague connections. Multiple students talked about how they found certain topics interesting because they had personal experience using the technology. These students also felt that fall term should adopt similar reforms.

\subsubsection{Sarah}

After fall and winter, Sarah wrote in the surveys how physics was important because it helps develop "well-rounded" knowledge and problem solving skills.

Physics is an excellent way to understand how things work and although not explicitly related to medicine, it is necessary for a well-rounded education in science.

Physics makes us learn new ways of thinking about problems and problem solving. It isn't easy but being a doctor won't be either.

It wasn't until spring term that Sarah mentions a connection with medicine.

Although biology has a clearer connection to medicine, physics is completely changing how medicine is practiced due to progressive technologies; therefore understanding how, magnetic fields, say, work is incredibly valuable.

Sarah clarified in the interview how her perceptions changed over the year. She

knew physics was important but had not fully appreciated how. While the spring 
course has helped her see that connection more clearly, Sarah said that she had been getting a better sense of that connection over the course of the whole year.

Interviewer: Can you tell me about how you view with the relationship between physics and medicine?

Sarah: I think they are very inter-related. And I touched upon this a little bit before when I was talking about how technology is becoming so much more advanced. And I mean without physics we can't have technology. And so things are becoming more and more dependent upon all these tools. ... And so because medicine is going more and more towards a technology-based field, I think it's going to be more necessary to know how those things work. Because I feel like, yeah you can read a manual and learn how to use something. But if you actually understand the functioning behind it, or the reasoning behind it's function, then I feel like it'll make you a better operator.

Interviewer: Did you always think that that was inherently important? How was your attitude towards it before taking a physics class this year, even the biomedical physics course?

Sarah: Okay. So originally I kind of thought like, yeah of course it's relevant because physics explains the world. But I didn't have a very good understanding of what that encompassed. And so I feel like now more so than before, I'm starting to see those kind of relationships. And like you were saying earlier like the inter-connectedness of the different sciences and the different things. I'm definitely appreciating it more than I was before.

Interviewer: And do you think that's kind of been developing over the course of the three semesters, like during the fall and the winter as well?

Sarah: Yeah, yeah absolutely. I mean just because we're learning specific things now that relate to medicine, I mean again physics kinds of explains everything. And I'm like at the very, very surface level of what it could be. And so yeah, I mean absolutely.

Sarah said in the interviews that the physics knowledge is valuable to her both because of the role it plays in the development of medical technology and the broader understanding she got from it. 
Sarah: Oh, man. Everything. All the things. I mean clearly having an understanding of the human body and then just physical reality. What I'm noticing more and more, and I've never really given physics much thought, but with technology growing the way that it is currently and how everything is based on, you know, nanotechnology and all this other stuff and the things that are very relevant for biology are also very based in physics. And so I think having an understanding here would, you know, is relevant, is necessary. So I think that just the more well-rounded a person is the better. That's why I want to do and MD as well as a PhD so that... Having a broader range of knowledge is going to be helpful for that purpose.

Sarah said she was anxious about having to take physics because she felt her math skills were poor.

Interviewer: Did you take either of the fall or winter term algebra based physics courses?

Sarah: I did. Both.

Interviewer: Okay. What did you think of those classes?

Sarah: Okay, so physics is kind of... Okay so going into physics I already kind of was biased against it. So part of that was that is because it's all math. And I used to think, because I hadn't taken math in like 10 years, I thought that I was bad at math. I was out of practice at math.

But Sarah then described how her interest in physics grew the more she studied it.

Sarah: So I think that physics in general is really interesting. The more I learn about it, the more interested I become in it. Because I see how things start to relate. And like, you know, why is it that when you drink out of a straw it works? You know, like really random... Yeah, really random things like that. And so the more I learned about it, the more I am interested in knowing more. 
When asked whether she thought any of the physics courses she had taken would be valuable for her career, Sarah replied that the concepts in spring term would be more applicable than those of fall or winter.

Sarah: Sure. Well I am personally more interested in the things we're learning this time. They do seem more applicable for a medical profession just because it is something that we utilize all the time. Whether it's, you know, with microscope use or, you know, the endoscopes or anything like that. Anything that allows us to get a better view into the body or, you know, be able to look at pathologies or what not. So I definitely think that this term is more applicable. It may be that that's the presentation because that's what we are being taught in the course. It's, okay how does this relate to medicine?

Interviewer: Was that discussed previously in other courses, like how things relate to medicine?

Sarah: No, not really. I mean we would talk about like how they relate to real life, but not necessarily anything to medicine in particular. So again it could just be because that's how I'm being presented the information.

Some of the topics that Sarah most enjoyed were things with which she had personal experience. When the interviewer asked her which topics she found most interesting, she replied,

Sarah: Well I think it's so cool how microscopes work. I mean I use microscopes every day. I work in a couple different labs and so I use them all the time. And then of course just in being in anatomy, we're constantly looking at histology. But it's just so interesting to me how the interaction between different wavelengths of light and visible light and, you know, infrared and ultraviolet and all that stuff and how that works. And looking at the interaction between, you know, the different kinds of lenses. I used to work in optometry and so I never really understood, like I mean understood the basics. Like okay you have a convex lens for farsighted people and here's what the rays do. But I didn't have any kind of real appreciation for it. And so having a better understanding, I feel it gets me more excited. 
... So I just think in general the more context I have to the tools that I'm utilizing, the more able I will be to use them.

Even though Sarah said she enjoyed the biomedical content because it was more relevant to her career, she also expressed that she would have appreciated a broader scope as well.

Interviewer: And what do you think about the biomedical content? ... Would you say that it made the course more interesting versus...?

Sarah: Yes, it has made it more interesting, because I think it's more applicable. But on the other hand, I would also like to have a maybe wider-world view of these concepts. Because of course it's super valuable that I know how a microscope works or an ultrasound works. But I feel like, even though this is what this is focused on, it would be nice to get just a little bit more information. ... But yeah it would be nice to have kind of a mix of both.

\subsubsection{Allison}

Allison was favorable to all three terms of physics. In fall term, she indicated that the concepts were foundational.

The physics that was covered in this first term was very applicable to many different fields as it covers basic aspects in life. I believe it provides a strong foundation for many different areas.

By winter term she had already made connections between physics and medical technology.

As I am preparing to enter graduate school toward a degree in Physical Therapy, I believe that many of the concepts relate to tools and equipment utilized by professionals in this field.

Allison reiterated her statements again in spring term. 
I think there is quite a bit of physics related to Physical Therapy and the information will provide good background for the profession and the concepts I'll be learning and working through later.

Allison indicated that she had an appreciation for the connection between physics and medical technology before coming into any of the courses. Although she clarified that she was curious about those connections but didn’t understand them.

Interviewer: Can you tell me how you view the relationship between physics and medicine? What was your attitude before taking any of these courses?

Allison: I think to some degree, to some degree just totally oblivious. But some degree was, you know, just kind of curious. You know, how does the MRI machine work? And when you get your, you know, even X-rays, and obviously that's not something we're going over in this class currently. But, you know, how does medicine get to manipulate science to better understand bodies? ... It's definitely there and being able to explore a little more has... I don't know, it's been fun.

Interviewer: Did any aspect of the course or the courses you had change your attitude about the relationship between physics and medicine.

Allison: Yeah, just in gaining more knowledge and a little bit better understanding. I definitely wouldn't say that I have more than a layman's understanding at this point still. But I would be able to talk about it and I definitely have a better understanding of the ideas behind how that science works.

Allison explained that even though she didn't think she would be using the technology discussed in the spring course, she felt that the knowledge would still be useful to her. Earlier she stated that she would not be using ultrasound imaging as a physical therapist, but could potentially use an ultrasound wand.

Allison: When I heard that there was a biomedical option I was 
definitely more interested in taking that with regard to, you know, like obviously I'm not likely to ever use an ultrasound machine as we talked about in class. Like maybe I'll use the wand for healing modality, but a lot of what we're going over isn't necessarily physical therapy related. But it's still really good knowledge. I feel like because it will, you know, there's still that opportunity for it to be something that relates, again like the ultrasound wand, to something that I will be using. And I like having that knowledge because when one of my clients ask about it, I'll be able to provide them with that information. I know what I'm doing with it.

Allison said that she enjoyed the biomedical focus but would also appreciate other types of real-world applications. Here, interviewer had asked Allison whether she would want to have had a similar biomedical focus in fall term. She explained that she had considered that before, but had been unsure of how to answer. She felt that having an instructor with a background in traditional physics was beneficial, because it allowed for both biomedical and non-biomedical topics to be explored.

Allison: It was really cool to be able to apply it to real-world things and kind of have that basis of physics. But it doesn't necessarily have to be biomedical like the whole time. I don't know. You know? I still think...

Interviewer: You would appreciate some other type of real-world things in this course and not just the exclusive...? Or do you think that's happening? Do you think little bit of both?

Allison: I guess, maybe... We have focused mostly on the biomedical and... Which is great. I think it's been really great. Especially sound waves and light waves and those kind of things. Like when you think about application in the real world, for me, I think my strongest interest is in the medical field and the technology and tools that they use. And maybe I don't feel like I'm missing a ton out on that, just in part because Dr. Dunlap fully recognizes that it, it's not his strong suit. The medical side is not his strong suit. So when he's like, "Hey, here's an example." He doesn't go to the medical side of it. He goes, "Hey, here's a bubble and see how this works." You know, so. I kinda, we're still kind of getting that. 


\subsubsection{Hailey}

Although Hailey didn't provide written comments on the spring survey, her responses in fall and winter indicate that she was doubtful about how much physics should be required of pre-meds.

I think pre med should be an equal amount of science and non science. Just because you are good at science and math, doesn't mean you'll be a good medical provider.

I think some of it is relevant to my future career as a doctor but I don't think taking a full year of physics is a necessity.

During the interview, she clarified that fall and winter term concepts were taught through idealized scenarios such as point particles. Hailey then explains that without real-world applications, she didn't see the value of learning the material.

Hailey: I mean of all the terms, this is my third term of physics, I think that this is definitely the most relevant to any sort of health care field going forward. At my work we do use the pulse oximetry and ultrasound and stuff like that. So it's been kind of cool being able to chat with my co-workers about the stuff that I've been learning. It's a good basis for things like that. Just kind of completing the circle, which is kind of the way that I prefer to learn.

Interviewer: Okay. Can you tell me what you mean by that?

Hailey: Yeah, so I think the first two terms were very general. Physics and lots of math, lots of point particles. That doesn't really relate to real life for me.

Interviewer: Idealized situations?

Hailey: Yeah, yeah. I would rather know like how this translates in the real world kind of thing. Rather than this kind of, and I think I have the disadvantage of being brand spanking new to physics as of fall term. But that seems kind of, why do I need to worry about point particles, or magnetic fields, or which way is this current running. Like I... Sell me about why I should care about this. But this 
term has been great in getting that buy-in from me and my interest, because it's relevant.

Hailey said in the interviews that by spring term she had begun to see a connection between physics and medicine. And by having that connection, she started thinking back to possible connections relevant to concepts from fall term.

Interviewer: Can you tell me, how do you view the relationship between physics and medicine?

Hailey: I mean, I think again, since I'm so new to this, if you had asked me in first term, I would have said there isn't one. But definitely as time has gone on I've seen that, especially in technology now-a-days, I would say like medical technology is... There's definitely physics happening there. I haven't taken any classes on this yet, but I would imagine that body mechanics, there's got to be some sort of ways to not injure yourself, your body. But definitely in technology, it seems very much based on physics.

Interviewer: Would you say that taking the spring course has really changed your view of how that fall is connected to that?

Hailey: Definitely.

Interviewer: What in particular from the spring course do you think has helped that?

Hailey: I mean, just being able to relate it to real life stuff that's easily accessible or things that people come across in everyday life. I mean sure we come across currents and circuits and stuff like that but you don't, I haven't had to deal with them that much. I know that it happens all around me but I'm not actually using that stuff directly. But stuff like how a pulse oximeter works. I use those every time I work. When we did our deliberative democracy talking about auscultation versus using these hand-held ultrasounds and stuff like that. I felt like that was a really good way to kind of tie-in kind of old school versus new school and kind of tie-in more of the physics stuff too, where we might see it in medicine.

Hailey indicated in the responses above that she had enjoyed learning about ultrasound and pulse oximetry partly because she has experience with those technologies. 
She reiterates this feeling when the interviewer asks her which topics she found interesting.

Interviewer: Where there specific topics from this course that you've found interesting?

Hailey: I like the ultrasound stuff, and the endoscopy stuff was kind $\overline{\text { of cool. }}$ What we're talking about now with the light intensity stuff seems kind of interesting. [referring to the pulse oximeter module]

Interviewer: What do you think it is about those things that makes it interesting?

Hailey: I mean just personal preference. Just personal. Just stuff that... I mean the ultrasound thing... Like just before I started this course I was working. And one of the girls at my work, we were cleaning ultrasound probes. And she was like, 'I wonder if it's the probe that costs so much or if it's the actual computer part and what about the probe makes...?' And I was like, 'I don't know. I'll tell you after I take this class.' And we found out it's definitely the probe. They're like 10,000 dollars... The pulse oximeter stuff. When I can go back to work and be like, 'This is how that works. Did you know that?' And some people are like, 'Uh, yeah. How did you not know that?' And other people are like 'That's really cool.' So just I mean personal preference.

\subsubsection{Leah}

Leah's attitude toward physics noticeably changes over the course of the year. After fall and winter term, Leah did not think physics should be required. Her survey responses indicated that she recognized that physics was a fundamental science, but that the courses were excessive. She also hints at the idea that physics is used as a weed-out course.

I can not see how knowing how to apply physics equations will ever be useful as a doctor. The fundamental Ideas are good for all people to know but a whole year this extensive is excessive. I think they just want to test 
us and see how well we can retain hard information but they shouldn't be using physics to do it.

By winter term, her attitude was beginning to shift. Although she maintained that physics shouldn't be required, her comment suggests that she is more in favor of the course than in spring term.

I kind of get why they want us to take this class as a premed., they want us to be well roundly educated. I feel like algebra based physics is useless for anything else. If they are going to require it, they should require calc based so it actually has meaning.

After spring term, Leah was very in favor that physics should be required. Like in fall and winter, her comment in spring suggested that physics as a fundamental science was valuable. However, she was much more adamant about this in spring term.

I think this course should be required for all people in the world. Physics is a part of everything we do and I feel everyone should have a good general understanding of what's going on in the world...

During the interviews, Leah talked about how her attitude toward physics has changed over the year. She came to see having a 'well-rounded' view to be beneficial.

Interviewer: Do you think that these classes were important for your planned profession at all? The fall, winter, and spring courses?

Leah: I do. I didn't at first. For most of first term physics, I was like, 'Why do I have to know this?' But as I've gone on I've just kind of realized that if you're going to be somebody with, you know, an MD or PhD, you should have well-rounded knowledge of the world. It shouldn't just be this one thing that, you know, maybe I'll get there and I'll be like, 'God, I'm so glad I learned physics. Everything just makes sense now.' Maybe not. But I think it should be something that you're aware of and know. Maybe all college students should know. 
When the interviewer asked Leah if anything she has learned will be useful for her career, she replied that spring term made those connections clear. Fall and winter terms may be useful as well, but since medicine wasn't emphasized, it was hard for her to tell.

Leah: I think that question is a little easier to answer for this term, because they kind of spoon-fed it to us. Like 'Here, this has to do with what you're going to do.' Now we're talking about lenses and the eye. So obviously this relates, because we're going to learn about human beings, everything in them. So I think that's kind of a hard question to answer about the first two terms, because it wasn't made as obvious to us. But I'm sure the electrical stuff, what am I going to do with that? I hated circuits. I don't see that ever being something where I'm like, 'Yay, let's use this.' But definitely first term, learning about forces and stuff like that at some point have to relate somewhere. I don't know exactly where.

interviewer: So you're skeptical whether the first two terms...

Leah: I wouldn't say I'm necessarily skeptical. I think I just.. I'm not in medical school. If you came back and asked me in eight years when I'm done. I might be like, 'Oh yeah, this goes here and...' But I don't have any of that knowledge yet. And so, it's easier to say about this term, because they gave it to us.

When asked about the relationship between physics and medicine, Leah talked about how physics underlies technology. She stated though that she wasn't sure just how useful that knowledge would be to practitioners.

Interviewer: So can you tell me about how you view the relationship between physics and medicine?

Leah: From what we've learned so far, mostly between like the instruments that we're going to use. ... I'm not sure how that's going to relate to prescribing medicine and stuff like that. So I'm not sure if it's more related to the people who are creating our instruments or us, you know, future doctors. 
When asked about whether the physics she learned will be useful for her future career, Leah was not sure. She stated that she did not yet know if it would be useful, but that because of spring term she was optimistic.

Leah: I think so. I just don't know. I don't know, cause I haven't really like been there yet. It's kind of a hard question to answer. I would have to answer based of what I think [doctors] do, but I don't actually know. I'm not in it.

Interviewer: And that's something that you have a hopeful expectation, that all three courses have made an impact on that? Or does the spring course have a different impact than the other courses or anything?

Leah: I think the spring course make me more hopeful that it will be connected. That there will be more things to... I kind of wish that we had a whole year. Cause I think it would have been fun to see them related to the other stuff if they can.

\subsubsection{Emily}

Emily was neutral about whether physics should be required in fall and winter. Her comments show that she thought physics knowledge was important because it is a fundamental science. However, she felt a full year was excessive.

I believe that taking this class will help me better understand the world around me and certain aspects of biological life, that I might not have understood otherwise. I also feel that in order to fully understand a topic, you need a comprehensive knowledge of that topic and because biology, chemistry etc. can be explained by physics, I think aspects of this class are vital to my future medical profession. However, I'm not convinced that the information covered in this course couldn't be condensed to a shorter, more medically relevant course. I'm also not convinced that I couldn't learn the relevant physical topics while in medical school.

In winter term, she stated that she couldn't see the relevance of physics. 
While it is likely helpful to understand basic physics, I had a lot of trouble drawing parallels with this terms material and my future career.

By the end of spring term, Emily was able to see how physics is important to the development of medical technology. She felt that first and second term had more biomedical content.

This course has really helped me see the connection between physics and medicine. I just wish 1st and 2nd terms were more related. I think having the biomed focus with the physics class helped me gain a greater understanding of the role physics plays in medicine. It also helped me see realized that somewhere someone understood physics well enough to develop these incredible diagnostic tools. Without an understanding of physics, we likely wouldn't have LASIK, ultrasound, centrifuge, pacemakers...the list goes on and on.

Emily state in the interviews that she wasn't able to see the relevance of physics to medicine in the fall or winter terms. The applications in spring term changed her opinion though.

Interviewer: Can you tell me about how you view the relationship between physics and medicine?

Emily: Before this class, I couldn't tell you. There was nothing for me that seemed relevant. After this class, I mean obviously with ultrasound. So if you want to do radiology, like it's all physics. It seems like it would be all physics. Definitely if you wanted to do optometry or ophthalmology. Like I totally get why you need to understand where the image is projecting. Is it behind the eye? Is it in front of the eye? Do you need a virtual image? Do you need a real image? And what type of lens to prescribe? I think, like that's all exciting.

Because she wasn't able to relate physics to medicine, Emily said she wasn't as interested in the material. Although she couldn't see the relevance of physics in fall term, by the end of the year she felt that there might actually be connections. Emily 
started to see this on her own while she was working.

Interviewer: Okay, did you think those classes in fall or winter were useful or...?

Emily: It was a lot harder for me to see why it was relevant. I love that about this class, because it's like, "Oh, physics might actually matter in my career." You know? ... I took physics in high school as well and it was never related to medicine either. And I had a pretty low interest in it then. ... I think in terms of generating interest in class, this has been a lot better for me.

Interviewer: Do you think that the fall or winter courses are going to be important for your medical school or anything like that?

Emily: I think some of it. You know I'm sure I'll, it'll be immediately apparent when I start to do it. But I shadowed an orthopedist and, you know, how you set a broken bone and the angle you set a broken bone at really matters. I was like, "Oh, that's a little bit of physics." And probably knowing what happened and where the bones went is probably, you know, good to know and that sort of thing. But this is really easy for me to see how it's useful. I love that about it. I would love first and second term to be all about this.

Emily explained that she took the biomedically-focused course because she wasn't able to see the relevance of physics to her career and she wanted to find out why it was required. She recognized that experts valued the knowledge, but couldn't see why until spring.

Interviewer: So you can tell me why you took the physics course with the biomedical focus?

Emily: Because I want to love it. I've always loved school, always. ... So I love learning and I love understanding about the world around me. So... But I took bio [referring to reformed physics course] because I wanted to know why it mattered, you know? Why is this required for medical school? Because obviously people understand that it's important or else they wouldn't require it for medical school. So there's got to be a point to all of the things that we've learned all 
year. But I just couldn't see how it was relevant until this term.

\subsubsection{Maria}

In each of the three surveys, Maria stated that she thought physics should be required because she will need it for her future career.

In the interviews, Maria states that what she enjoyed about the biomedical focus was learning how medical technology works.

Interviewer: What did you think about the biomedical content, overall through the class?

Maria: I thought it was absolutely wonderful. It was very relatable. I was excited to see how the instruments actually work because...yeah with ultrasound sound you get white images and black images, but I didn't understand what any of that meant or why. And so it was exciting to learn about those.

Even though Maria indicated in her survey responses that she knew physics would be important for her career, she stated in the interviews that she did not see a connection to medicine until spring term. She was surprised to find out that biomedical experts retain physics knowledge and use it in their careers.

Interviewer: Can you tell me about how you view the relationship between physics and medicine? Maybe like start off with before you ever had physics classes, did you ever connect those two things together?

Maria: No I did not. ... And I was actually really blown away by this term. How some of our flip it models have some of these doctors from a local hospital at OHSU doing interviews and they're like really talking about these tools that they use every day and how it is so physics heavy. And it's not like something that you just learn and I'm a doctor now I'm just diagnosing symptoms. It's like really a 
part of their everyday life. So I actually didn't realize how much it was retained. I mean, I don't know if I was ever some ultrasound technician or endoscopy doctor, if I would really think about the physics of it but they all clearly did. Cause they just did it really naturally. So yeah, it was everywhere. It was everywhere and I didn't realize it.

Interviewer: So did that happen at all in the first or second term, do you think?

Maria: With medicine?

Interviewer: Yeah, did that impact at all? Did anything really...

Maria: No. Not a single time.

\subsubsection{Cathy}

Cathy replied that physics should be required after winter and spring term, however she did not see a strong connection to her future career as a physical therapist in those classes. The value for her seems to have been more about problem solving and a better understanding of the world. In winter Cathy wrote,

Knowing math and being able to problem solve is important. The engineering biased material was not as necessary.

And after spring she wrote,

Some content applicable to PT. Most not but still good for a basic understanding. A good class to test students' ability to understand physical world and drive to succeed (get a good grade).

Although she did not respond to the interviews after fall term, Cathy indicated in the interviews that she viewed fall term favorably because she thought it was more widely applicable. Spring term seemed less useful. 
Interviewer: Can you articulate what kinds of knowledge, skills, or experiences you think are important for being a physical therapist?

Cathy: I think levers. A lot of the first term stuff was really good in physics for physical therapy specifically. That's about it. Now is good for the mostly med focus, like doctors. It's not great for physical therapy unless you get more specialized and start working with ultrasound and things like that.

Cathy later stated that what was valuable was getting a better understanding of the world. When she was asked if there were any concepts she learned that would be useful to her she replied,

Cathy: Well for me, I do enjoy just knowing and learning about it because then the physical world you can just see all of the way it pertains and then how everything works. For like, my field or what I'm really going to use in my career, no not really.

\subsection{Should spring term be required? - Students that were neutral}

These students reflected some of the attitudes seen in the survey data. Henry thought

the reforms were an improvement, but remains skeptical of how the knowledge will be of practical use in his career. Noah also liked the reforms, but has a greater interest in physics as a foundational science. April and Alan think the reforms are an improvement over the previous terms, though they provide less detail about what impact that had on them.

\subsubsection{Henry}

Although Henry did not respond to the survey in fall, his neutral responses in winter and spring seem to be mirror feelings of other students. Physics is a weed-out course 
but it does help develop problem-solving skills. In winter, Henry wrote, "It's a good way to filter out people that aren't willing to work hard." And in spring he simply stated, "Builds reasoning skills."

Toward the beginning of the interview, Henry restated these ideas. When asked about his opinion of the fall and winter terms, Henry stated " They were difficult and just another obstacle in my way. That was exactly how I see them. "He told the interviewer that he was considering two career tracks, physical therapy or public health administration. The interviewer then asked what kinds of skills or knowledge will be useful for those fields.

Henry: Yeah, I would say physics not so much. I see the reason why they put physics inside like the course requirement as more, I think, to develop a ... problem solving skills for the students, you know. And also a way to like weed students out that aren't serious about actually working hard to get to their degree path. Because I can't see, even with this new course, physics being used in my career path. It just doesn't happen. Like they just don't use physics. ... I've worked in physical therapy clinics for some time now. And I'm still not seeing how any of this is, how any of this is really applicable. But I see why they're doing the physics course in the sense that it's used to develop ... problem-solving skills and to weed students out.

Henry was asked whether anything from fall or winter was going to be important to his career. At first he seems to be making some conceptual connections, but soon discounts their practical value.

Interviewer: Was there anything in like particularly the fall or winter term courses that you thought were important for what you're going to be doing in the future?

Henry: I could see some...

Interviewer: Other than problem-solving I guess. 
Henry: Yeah, problem solving and things like that. Other than that

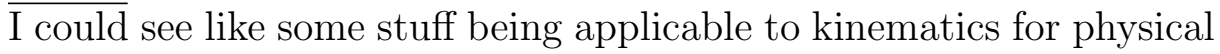
therapy. But I mean in the real world, not really, from what I've seen in clinics, which you know I haven't had a ton of experience. I've had several hundred hours which is like a good amount of experience. But not like I'm not an expert. But I have experienced enough to like, I haven't seen physics used yet.

He restated this idea when asked whether the fall term should incorporate more biomedical example, including topics specific to physical therapy.

Henry: Yeah, I think they could definitely make it like applicable to physical therapy when it comes to load bearing and things like that. I mean, in reality in the clinics, you're not really going to be using equations like that. But it would make me more interested and it may make other students more interested in the material through that.

Henry recognized that physics is important to medical technology but doubts that in-depth knowledge of the underlying principles are needed by practitioners. The interviewer asked Henry whether there was a relationship between physics and medicine. Henry replied,

Henry: Oh definitely. I mean the medical equipment is based on principles of physics, you know. Like without physics we wouldn't have our equipment. So yeah. I would say medical equipment development. But I mean like a physical therapist isn't necessarily developing an oximeter, you know, to measure the oxygen level in your finger tip. You know, it's stuff like that. Or the optometrist may not necessarily be inventing the lasers used in the LASIK procedure.

Interviewer: Right, so somebody else is pulling those things along.

Henry: You know I'm sure they have some part in it. And maybe they have a bigger part in it than I can comprehend. I'm not sure, but it seems like there's an engineer and there's a doctor. And yes, they probably do collaborate. I hope they do. But I would say the engineer would be the more the technical person doing the actual 
thing. And the doctor would be like, "Hey this is what we want. Can you make it happen?"

Another issue for Henry, is not a disinterest in physics, but rather using his resources efficiently in school. When asked whether he thinks about the physics of everyday life, he replied,

Henry: Yeah, I mean I love, I like physics. I actually do find it interesting. It's like by me saying that it's not applicable to my career path... I'm in college to have a career. I'm not here to just have fun. ... I enjoy learning about physics. I enjoy learning about the natural world, and like how science has studied that. It is interesting to me, but I'm here for my career. Like you know? I like to paint too you know? But I'm not taking those courses because it's not required.

Interviewer: Yeah, you're not going to pay money to go paint some...

Henry: I'm not going to pay thousands of dollars to go do painting. I mean luckily I have a scholarship where I don't have to pay tuition, however I'm not wasting my scholarship. I'm not here to waste it.

\subsubsection{Noah}

In fall term Noah agreed that physics should be required. His justification was closely tied with the idea that physics is a foundational science. "It is important to understand the fundamental nature of the universe. Physics like chemistry is an important piece of that understanding." By the end of spring term he maintained that attitude, but expressed that the in-depth detail of the courses might have been excessive. " An understanding of physics is important for studying science but an in-depth exploration doesn't seem entirely necessary for my profession."

Noah explained early in the interview why studying science was valuable to him. 
Noah: I think that the great thing about taking the variety of science classes is that it's given me a good foundation in understanding the principles of science, the principles of research, the principles of how that research is taken and then applied to the sciences. And you know everybody talks about evidence based practice in medicine. And I think to be able to use evidence based practice, you have to have a good foundation in understanding the sciences. ... You know, in some ways taking all these classes, to me, are just hoops that I have to jump through to get into grad school but at the same time, I'm also getting a good foundation in the sciences. So hopefully I can understand them well enough to be able to, you know, read literature and be able to apply it to my practice.

Noah didn't feel that the content knowledge would be valuable until later on. What he felt is important for him now is a broad understanding.

Noah: Yeah, you know, I think the specific concepts will probably be applicable if something similar comes up later, later on down the line as I'm as I'm reading literature, as I'm exploring something tied to my profession. And it might draw on some of these concepts. So it's great to have an understanding of what these basic concepts and ideas are. But to me, I feel like I'm trying to take a broader general sense of what physics is, what the study of physics is and trying to kind of understand more practical applications for it. Rather than necessarily holding on deeply to the to the specific concepts that I'm learning.

Noah stated that the course reforms have impacted his views on how physics is related to medicine and that he did not see an application of physics in medicine until spring term.

Interviewer: Could you tell me how you view the relationship between physics and medicine?

Noah: You know obviously there is a lot of physics that goes into the technology that's used in medicine. And that's something that's that I've really taken away this term especially through optics, things used in vision. As well as obviously medical devices like an ultrasound or 
even a microscope or, you know, we've been studying lasers in class. And how those are used to treat a body. And I'm sure you could probably look at every piece of medical equipment and pull out the physics that was used to make that happen.

Interviewer: Okay, sure. Can you tell me something about your attitude about physics in medicine before taking these physics courses? Was there...?

Noah: I would have never thought about the application and the relationship between the two of them.

Although what was important for Noah, was not how physics is used to develop medical technology specifically. Rather he appreciated learning how physics underlies technology in general.

Noah: I think that was the biggest thing I took away, is being able to see, ... and I mean obviously, applying it to medicine because that's the, that's the field that I'm going into, and a lot of my classmates are going into it as well. But, you know, obviously just taking the pieces of physics and how they can be applied to anything in our world, you know? Like the microwave or whatever it is. What are the kind of foundational principles that go into those? That's to me really fascinating. And, you know, we use tools in everything that we do and obviously those ideas and those principles came from somewhere. Maybe they were created without people necessarily understanding those principles. But to me that's been the biggest takeaway, is just like understanding when I'm looking at a tool or using the tool, all of the ideas in the background work on very simple principles that are built up to make it work.

At the same time, Noah felt that the scope of the course was too narrow.

Noah: I mean, we spent weeks and weeks on ultrasound. And so it just kind of got to the point where I was sick of hearing about ultrasounds, especially since I just don't really care about ultrasound. And from that aspect, from that point I would love to get a larger variety of things that we're studying, you know? We've studied ultrasound. We've studied I guess laparoscopic surgery. We've studied 
basically things to do with the eye: lenses and optics and that kind of thing. And so in the whole world of medicine we've only really touched on, you know, three little pieces of it. ... So I guess it's nice to have the concepts but the field that we're studying them in is so narrow, that they just kind of they start to confuse each other.

Noah clarifies that he can get lost in the details when studying one application. Having multiple applications helps him grasp concepts more easily.

Noah: Attenuation, resolution, all of these different things looking at all of the different things that an ultrasound will look at. Sometimes they become so similar to me when I'm thinking about them, that it's hard to tell the difference. Whereas, you know, when I'm thinking about the Doppler effect rather than thinking about imaging blood, I can think about, you know, using it to understand the speed of rain particles in a cloud. ... And so, you know, for me, if I'm relating it to things in the real world then ... if I can relate them to separate things, it's sometimes easier to hold on to them rather than all being related to, you know, imaging like one little square and all the different little details that go into it.

Because Noah felt the course was so focused on a small number of medical applications, he thought that he was not getting the same broad knowledge he got from other terms.

Interviewer: So compared to the fall and winter terms, you're learning about the same amount or...?

Noah: I feel like I've learned a lot less this term.

Interviewer: Okay.

Noah: Yeah. I feel like I've really learned a lot less. Yeah.

Interviewer: Okay. And by a lot less, do you mean like physics or...? ... What did you get more of in the previous two courses that you're not getting as much of in this course?

Noah: I feel like we covered a lot more concepts and ideas in the previous terms. ... Just in general, I felt like we covered a lot more 
concepts and ideas. We got through more things each day in class than we're getting through now. And so, you know, I feel like now we're kind of digging into stuff a little bit deeper. But before we ... We got more a general knowledge about a lot of different things.

\subsubsection{April and Alan}

April and Alan provided less detail in the interviews about their attitudes toward physics and medicine or how that had been impacted by the spring course. Neither student claimed that fall term physics was very interesting. It was not until the reformed course in spring that they saw some connections between the content and medicine.

Alan was asked whether he thought fall term was interesting or useful. He replied,

Alan: As far as interesting, I'm not sure anyone finds basic kinematics interesting. I mean, I'm glad it exists. I'm glad someone knows how far a baseball goes when it's dropped from a blimp or something. But it's wasn't personally interesting to me. But I respect is a science and I'm glad someone knows it.

When asked what kind of knowledge would be useful to him for his future career, his response suggested that physics is a fundamental science. He also stated that some topics from spring term might be more relevant.

Interviewer: Is there anything like knowledge-wise from your postbacc education process that is going to be most important?

Alan: O-chem definitely and then also physics feeds into that. They always say chemistry is physics and I'll take their word for that. ... But some of this stuff that were using here, like when we're talking about LASIK and Optometry. That seems like that might actually come up. 
April seems to share similar views. She did not see a connection to medicine until spring term.

Interview: So can you tell me about what was your what was your view on the relationship between physics and medicine? In particular like before taking any of these courses, did you think that they were connected at all or were they...?

April: I had yet to make a connection, like through like pre-med experiences, I had yet to make a connection. ... It felt like just a mandatory requirement.

Interview: Sure. And like did any aspect of the courses that you took change your attitude about that?

April: Yeah, I think the third term really did. In second term I like started to... Second term I like started to see like a little bit of the connections like with that, like researching ... certain surgeries. But it wasn't until the third term that I really made, like it didn't even occur to me that a radiologist did anything with physics.

Both Alan and April felt that the biomedical experts lent authenticity to the content. Although in April's response, she draws a distinction between the content from the biomedical experts and physics content.

Alan: It seems authentic. I mean I went to public schools in Ohio and not to bad-mouth it or anything but we'd get the inauthentic kind. Where like Timmy is, you know, an astronomer and he wants to add one plus one. And it doesn't have anything to do with astronomy. You just threw the word in there to make it seem fancy. But in this, it seems that you have actual, real doctors from one of the premier research institutions coming in. And they work it in very well. It seems like it's medically oriented. I mean I obviously don't have the knowledge to say $100 \%$, but if I got somewhere and I found out it was a fraud I would be shocked.

April: I feel like, I mean the stuff that he's actually lecturing about 
his more physics. ... I'm not going to like sit down and draw like an object and my rays and my mirror and stuff like that as a doctor. But I do think that the stuff, at least in the videos, is pretty authentic. ... They're so many videos on LASIK that they had an ophthalmologist do. And he was really in-depth and I was like, it was so far above my head. So I do think that the content is genuine. And like you could, like those are all doctors talking about their field. So I assume, I mean granted I don't really want to go to those fields but I do think it is like real material.

\subsection{Should spring term be required? - Students that disagreed}

Neither Ryan nor Alex felt that physics should be required after taking the reformed course in spring. Both students felt that the biomedical reforms made the course more relevant to their future careers. Despite this, Ryan still had doubts about how useful physics knowledge would be on a daily basis. Alex felt that the course did not actually value the biomedical content and that the topics lacked depth.

\subsubsection{Ryan}

Ryan's explanation for his ratings on the fall and winter surveys clearly fit in the 'little to no need' categories. In fall he said, "No, because I don't see the practical use of this in medicine." And in winter, "I don't see how physics apply to medicine." His response in spring was more difficult to categorize, "Not everybody wants to be a doctor."

In the interviews, Ryan said that he had apprehension about taking a physics course even though he enjoyed learning about physics concepts outside of school. When he was asked about his view of fall and winter term, he replied, 
Ryan: I was neutral about it. It was another class that I had to take it. Before taking it, I was dreading kind of taking physics. I like physics. I like listening to like Neil DeGrasse Tyson talking about, you know, random stuff. I like that. But in terms of learning these things, I don't think I'm as excited. So going into it, I wasn't too excited about taking a physics course. So it was just another class that I had to take. It wasn't anything that I was either excited about or...I guess I was less excited than my biology classes I had to take.

Ryan had hoped that a course with a biomedical focus would have been more interesting, because he wanted to see the practical application of physics.

Interviewer: Can you tell me why you took the physics course with the biomedical focus?

Ryan: I thought it would be more interesting if I could take a class that would be more relatable in what I'm interested in, which is medicine. That's the main reason. Before I took the course, I know that physics has a lot to do with medicine and all that stuff. But being able to apply it was something I thought I was going to enjoy.

When the interviewer asked Ryan about the relationship between physics and medicine, he said the importance of physics is in its relationship to other sciences and to medical technology. However, Ryan is unsure whether the content will be of practical use for his career.

Ryan: As of now I know that physics relates to medicine as well as many other things like chemistry, biology. Physics relates to a lot of the technology that you use and how they work and why they work the way they do. But as of now, I guess I'm still not that excited about it. I feel like that's something that maybe when I get into medical school and I actually start using these techs I'll be more interested in exactly how they work.

Interviewer: Okay, you expect that's going to be a part of that medical school experience?

Ryan: Maybe, maybe not. Maybe...I'm not sure...I have to look 
at the curriculum for school. But I don't expect it to. I expect it to be more useful during residency or some fellowships that you decide to do. Because if you want to be a radiologist, you definitely need to know how an x-ray works and how an ultrasound works and how different imaging technology works. ... I'm sure they'll go over exactly how does the machine work and what makes it work. But in medical school, I don't think they go into too much detail about how an ultrasound works at the level we're learning it now.

Later in the interview, Ryan reaffirmed that the biomedical content is relevant to his planned career.

Interviewer: Okay. Do you think the biomedical content seems relevant to your career plans?

Ryan: Oh yeah. Definitely, yeah.

Interviewer: Do you think that makes the physics more relevant to your career plans or just that biomedical content might have something to do with it?

Ryan: It makes it more relevant to my career plans.

However, he soon qualified this by restated that he was skeptical of whether the physics knowledge he learned in class will actually be used. The interviewer asked Ryan whether he thought the biomedical content of the course was authentic.

Ryan: It's just that it's hard to imagine when you're giving someone an ultrasound that you're ... mathematically going to a piece of paper and saying, 'Okay, that's how fast the blood is traveling.' It's usually that now a days you just have somethings that just pops up in front of you. The computer does all the work and just tells you. So I just can't see that someone will sit down and, you know, do math problems to figure it out. Even for an optometrist, I'm sure that they have some sort of computer program that they can plug in and the information just spits out. And I know it's important to know those numbers were generated. ... Maybe in a world where we don't have technology to calculate all that, then it would be more relevant, but... 
Even though Ryan hoped that the reformed course would show more applications of physics to medicine, he indicated that having the reforms was not always as enjoyable.

Interviewer: Do you feel that you're missing out on other topics that couldn't be taught in this course because there is a biomedical focus?

Ryan: Yeah, I actually talked to someone else who was in my lab who was in the normal physics. And he said that they were going to touch on quantum mechanics. Yeah, I would like to hear about that. It would be interesting to hear how that relates to... I don't want to hear that how it relates to medicine. I want to hear how it relates to the universe and the world that we live in. So I'm kind of bummed about that when we get to that point. And I think I did see in the manuscript that we do touch on it, but I'm actually kind of bummed that it's actually geared towards medicine. I haven't read it yet, but I was hoping that part... I'd be glad if he deviates from that and just talk about how it relates to anything.

\subsubsection{Alex}

In his survey response, Alex makes it clear why he didn't think fall term should be a requirement.

I think there should be a physics class for medical student, that way we could focus on material important to the human body. And less time on why Janie (since her waist is 30 inches) roles faster than Jill (whose waist is 50 inches) and they weigh the same... I find the material tedious.

Even though he completed a course with exactly this kind of focus, Alex still thought that physics should not be required at the end of the year. He justified his survey response at the end of spring term by saying, "Ochem helps me sufficiently to analyze complex ideas. I don't really want or need physics."

When he was asked about his opinion of the fall and winter terms during the interview, he reiterated his sentiment from the fall survey. Physics courses should 
have more biomedical content. Alex also felt that he might have been affected by opinions from other students.

Alex: So the thing about physics is there's not a lot of encouragement within the student body to see it as important. So a lot of students have a lot of negative things to say about it. So I think that it's fair to say that my viewed was tainted by a lot of the negative comments. But it is also at times very difficult to see it as useful. Just because, you know, we're learning about how to tell how fast a wheel is turning and you know I'm not sure that I'm interested in that.

Alex stated that the lack of relevance was one of the reasons he took the biomedically focused course. The interviewer asked Alex what his expectations were coming into spring term.

Alex: So I hoped that it would be more interesting because it was being applied to like, ... I'd hoped I wasn't going to be asked, you know, a dog is falling from a tree. What rate...dit-duh-duh? Where I'm like. "I don't really care." You know? I was really hoping more to see more like, the blood is going at this rate and duh-duh-duh. I was hoping for more of an in-depth kind of, and maybe that was unrealistic for a 200 level course, I think. Sort of get a little deeper into the ideas of why things are happening. And being to apply it to physiology really.

Alex stated here that he was looking for not only biomedical content, but "indepth" content. Later Alex describes how he approved of the course reforms and would like to see similar reforms in other terms, however he was not convinced the reforms were sufficiently extensive. He did not see the kind of in-depth content he was hoping for.

Interviewer: Do you think the biomedical content has made this course more interesting or just...? 
Alex: For certain. For certain.

Interviewer: Okay, so there has been a positive aspect?

Alex: There has been. I think that it needs to be more part of the curriculum as far as what is valued by test standards... In order for it to be valued by the students.

Interviewer: Do you think that the learning about that biomedical content is relevant to your career plans for going into medical school or do you think that's...?

Alex: Yes. [hesitantly] It is valuable. I think that I'd like, I wish... And granted this is a survey course, but I kind of wish we were spending a little bit more, in-depth on some things. If we're going to talk about something, let's talk about it, you know? Maybe, and in that sense as a teacher, maybe picking... I don't know how many subjects are in fall term but let's say there's 40 . Why don't we pick 10 of them to focus on the entire term? Where you're like deep, deep, deep, and you're like, "These are what we're, it's not that everything else is unimportant, but this is what we're digging into." Put the biomedical concepts in there, put everything else. The other 30 ... You know basically if I give you this exam it's going to be straightforward, just kind of almost like a plug and chug understanding on a basic sense. And these ten things, like this is worth digging into. I would prefer that very much.

When the interviewer asked Alex what topics felt most authentic, he said the endoscopy module felt the most authentic because the class spent more time on it.

Alex: Well you know that was the beginning of term. We had a little bit more time and I think that we went over it more. And we kind of, we got a little bit more in-depth into it. I feel like the videos were more, more interesting. And that's not just cause I liked endoscopy more. Frankly, it was difficult. But I feel like the videos were less chitty-chatty. Some of the later ones were a little more chitty-chatty.

Finally, Alex makes it clear that he did not feel that the biomedical content was being valuable to the course because grading was based solely on traditional physics content. 
Interviewer: So there's quizzes in his classroom?

Alex: There are quizzes purely of the equation based information. So like the checkpoints will have like ideas often. Sometimes equations but a lot of them are ideas. And like, within like the video they talked about ... information that is interesting, but we have a cumulative final, as you probably know. And nothing that is biomedically, that is medically specific is going to be on there. Only physics. So basically it just kind of boils down to last year, you know, the last two terms. That's what it boils down to.

Interviewer: So despite all of the other content and the way it's presented...

Alex: In the in the end, it's not presented as important in my opinion. Because if you're not going to test me on it, that says to me that you don't value it.

Interviewer: So it's been your experience that the quizzes haven't focused on that aspect at all? It's mostly just the physics stuff?

Alex: No nothing.

Interviewer: And that you have a clear expectation of the final is going to be on that same subject?

Alex: Yes, very clearly. He told us first day of class that if it's medical, which means he's not going to know a lot about it, it's not going to be on the exam. 


\subsection{Discussion}

Every student that participated in the interviews stated that the biomedical focus of the reformed course in spring was more relevant to their future careers than the traditional fall and winter courses. Although the impact this had on their views of physics instruction varied. Many of the reasons the students cited in the interviews were also seen in the previous chapter.

Most students stated that they did not see a connection to medicine until spring term. Sarah said she knew there was a connection, but did not feel that she really appreciated it. April stated that it had not occurred to her until the reformed course that radiologist used physics. Maria was surprised by how the experts in the videos actually used physics in their work. Alex thought the biomedical focus made the course more interesting, and wanted the course to put greater value in learning the medically-related material.

Students said that they found it interesting or enjoyable to study the physics behind technology they actually use in their work. Sarah said she was interested to learn about microscopes and interactions of different types of light. Hailey enjoyed talking about pulse oximeters and ultrasounds with people at her job.

Some students felt that their appreciation for physics had grown over the whole year. Sarah stated that, while she was hesitant at the beginning of the year, the more she studied physics, the more she interested she becomes. Emily and Leah both said that by the end of the year they could start to see applications of fall term physics in medicine.

Ryan, Henry, April, and Leah all expressed doubt to some extent exactly how useful physics skills will be on a practical level even though they all recognize that 
physics is essential to the development of medical technology. Each of these students made a statement that the kind of calculations they do in class would not realistically be performed in the field. Henry and Ryan make it clear that the in-depth knowledge they gained in class was less valuable to them because it was not needed to operate the technology. Although the course reforms were not intended to prove that all of the knowledge and skills learned in a physics course are directly relevant to the dayto-day tasks performed by medical professionals, it seems that some students might have interpreted it as such. This suggests that future versions of the course should make that clearer to students.

Many students stated that they appreciated the broad scope of the fall and winter terms. Sarah and Allison suggested that the reformed course should include biomedical and non-biomedical examples. Noah felt that he learned less in spring term because of the narrower biomedical focus. Ryan said that he thought his was missing out on other topics like quantum mechanics.

Every student claimed that the biomedical focus had impacted their views of physics in some way. For some students, the reforms built on an appreciation that already existed or had been developing. For other students, biomedical application helped them to understand how physics can be valuable to them in a way they did not see in previous terms. Some students appreciated the new focus, but were not convinced the knowledge would be significantly more useful to them in the field. Some students supported the reforms, but did not want the broader scope of traditional physics to be completely replaced. 


\section{Conceptual Understanding and Contextualization}

The purpose of these quizzes was to assess whether the reformed curriculum had an effect on students' conceptual understanding and contextualization of physical phenomena. The prompts for the quizzes were presented in an open-ended manner so as not to influence students' conclusions about how the different phenomena were applied outside of the classroom.

Each section in this chapter details the data for each of the quizzes given. The intended, expert interpretation of the prompt is given at the start of each section. This is followed by an analysis of students' conceptual understanding and real-world contextualization of the physical concept.

Based on the analysis in this chapter, the reformed curriculum does impact students' contextualization of physical phenomena. However, the open-ended nature of the quizzes makes correlating contextualization with conceptual understanding difficult. 


\subsection{Doppler Shift}

\subsubsection{Illustration Prompt}

The illustration depicted a particle with a velocity $v$ moving away from a source of sound $S$. The description given to students stated, "The image depicts a sound wave interacting with a particle in motion." This was followed by the general instructions shown in Fig. 3.7.

The image depicts a sound wave interacting with a particle in motion. Using the illustration below:

1. Complete the diagram by drawing the path and wave fronts of the wave as it interacts with the particle. Include as much detail as you can including descriptions of the physical principles that predict the path of the wave. ( $0.5 \mathrm{pt}$. for attempting a solution and description $+0.5 \mathrm{pt}$. for a correct solution and description)

2. BONUS: Describe at least one real-world application of this physical principle that is used outside of a physics classroom. Include as much detail as you can including describing how the principle is used.

( $0.25 \mathrm{pt}$. for a correct description of the real world application)

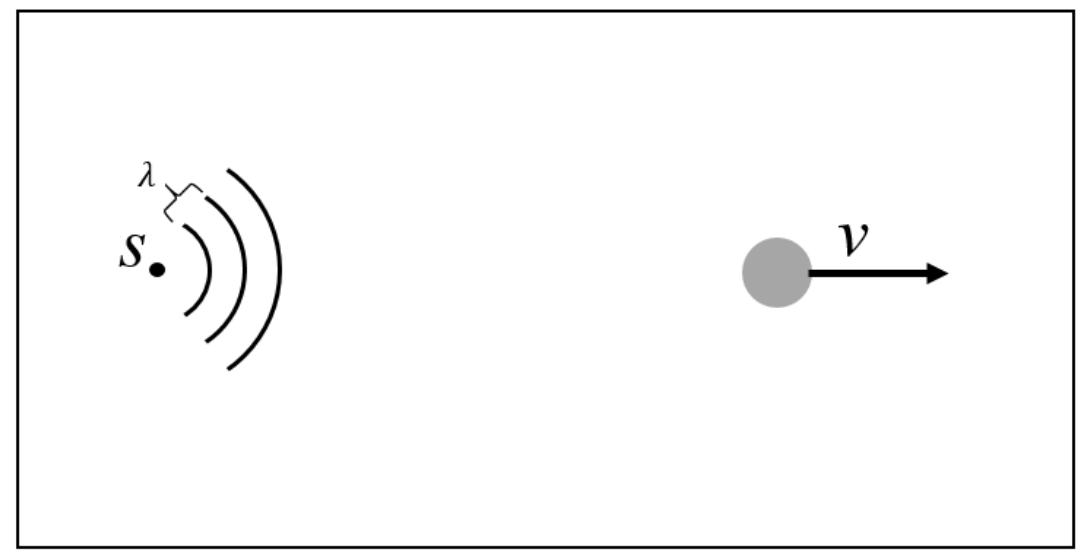

Figure 7.1: Illustration given to students for the quiz on sound waves and Doppler shifts.

As the particle is moving away from the source of sound, the frequency observed by the particle will be lower than the original frequency. This is described by the 
appropriate form of the Doppler equation

$$
f^{\prime}=f \cdot \frac{v_{s}-v}{v_{s}}
$$

where $f$ is the frequency of the original sound, $f^{\prime}$ is the frequency observed by the particle, and $v_{s}$ is the speed of sound.

One possibility in this scenario is that the sound will reflect off of the particle and travel back toward the sound source. In this case, the frequency observed by the source undergoes another Doppler shift that further lowers the frequency as described by the equation

$$
f^{\prime \prime}=f^{\prime} \cdot \frac{v_{s}}{v_{s}+v}
$$

where $f^{\prime \prime}$ is the frequency observed at the source of the original sound.

\subsubsection{Conceptual Understanding}

The prompt given to the students explicitly stated that the waves in the diagram represented sound, though some students gave alternative descriptions. One student from the reformed course and three students from the traditional course described the waves as electromagnetic radiation. These four students are excluded from further analysis to reduce the possibility of confounding factors. After also subtracting absent

quizzes, this leaves 93 students from the reformed course and 44 from the traditional course.

Although Doppler shifts in sound waves were covered in both the traditional and reformed courses, many students did not mention this phenomenon in their responses. $59.1 \%(\mathrm{~N}=55)$ of the students from the reformed course and $38.6 \%(\mathrm{~N}=17)$ of the students from the traditional course mentioned the Doppler effect in their response. 
Many of the other students gave descriptions having to do with more general concepts including wave propagation and sound intensity. The majority of students who described the Doppler shift correctly stated that the frequency observed by the particle is less than the original frequency. Only one student from both course incorrectly stated that the observed frequency was higher.

Of the students that mentioned the observed shift in frequency, 42 from the reformed and 11 from the traditional course explained their response with the Doppler equation. Once again students from both courses were highly likely to give the correct version of the equation. Only three students from the reformed course wrote an incorrect form of the Doppler equation. There was no relationship between these responses and the course reforms, $\chi^{2}(1, N=53)=0.83, p=0.36$.

The frequency of the reflected wave was described in only a subset of the responses. Figure 7.2 show the distributions of students' depictions of interactions between the sound waves and the moving particle. The majority of students drew a passive interaction in which the sound waves either end before reaching the particle or pass over the object unaltered. A smaller number of students drew the waves diffracting around the particle on the far side, opposite to the source of sound. $39.8 \%$ of students in the reformed course and $34.0 \%$ of students in the traditional course drew reflected waves or a combination of reflected and diffracted waves. There was not a significant relationship between the type of interaction and the course reforms, $\chi^{2}(4, N=137)=3.24, p=0.52$. 


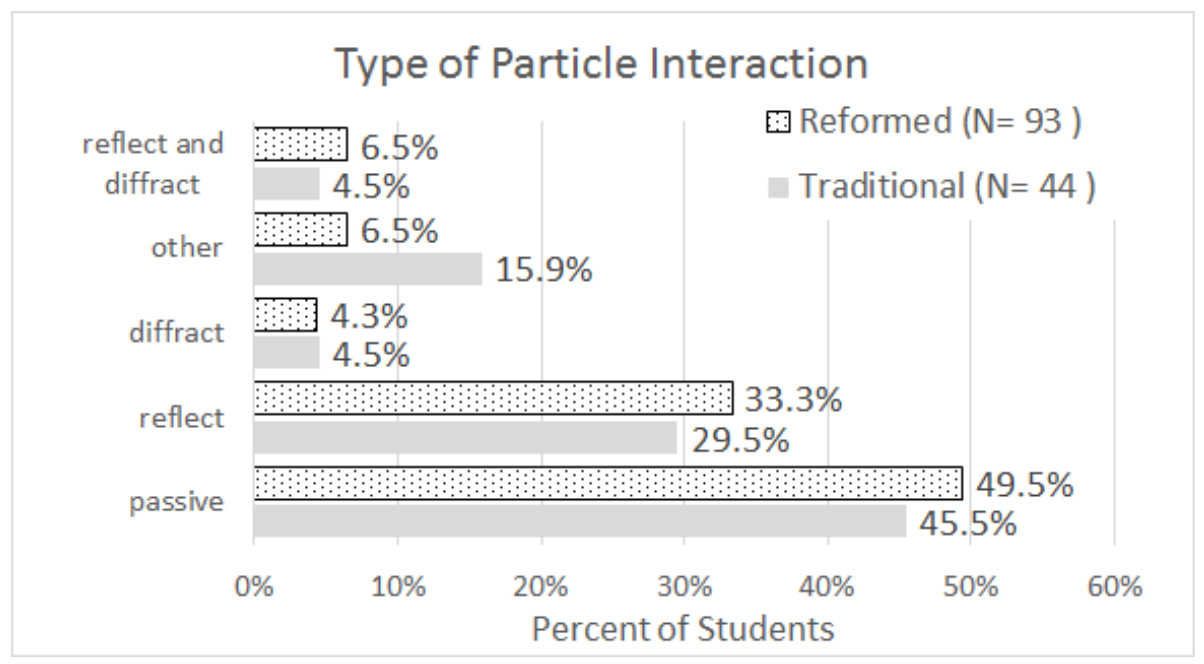

Figure 7.2: Distribution of the types of interactions between the sounds waves and the moving particle as depicted by students for the prompt in Fig. 7.1.

Of the students who depicted a reflection, only a few also described the Doppler shift of the reflected wave. The small sample population does not allow for meaningful comparison between the cohorts.

Although students in the reformed course were more likely to describe a Doppler shift, a large percent of both classes failed to do so. It is possible that these students interpreted the significance of the vector in Fig. 7.1 not as suggesting a Doppler shift, but rather simply a greater separation between source and observer. Many students described how the intensity of the sound, as heard by the particle, would decrease as the particle moves away from the source. This is true, but was not the intended purpose of the velocity vector.

It could be possible that students are conflating these two ideas. The intensity and frequency of sound observed by the particle would be less than at the source, but for two different reasons. The intensity decreases as the wavefront is spread out over an increasing, spherical surface area. The observed frequency decreases due to the Doppler effect. One of the students who participated in the interviews, who will be 
called Emily, did conflate these ideas.

Emily: And then I don't know if this is a hearing object or not, because then you'd have like a Doppler situation.

Interviewer: Sure, let's assume it is. What do you mean by that?

Emily: Well so, I know that, I think that I know anyway, how this object, person perceives the sound is based on the relative velocity or non-velocity if this isn't moving. So that's all.

Interviewer: So let's assume the source isn't moving.

Emily: Isn't moving. So if this has a velocity away... I don't know. I'm not sure that I know what it sounds like to this person. I think when the sound wave catches up it'd be much quieter than it is here at the source.

Interviewer: So quieter. What does the level of quietness have to do with where it's at?

Emily: Well probably nothing. Well because as you approach a point source I would assume it would sound louder.

Interviewer: Okay the closer you get, the louder it will get?

Emily: In decibels. I would assume. Because if you walk up to a speaker, it's much louder than when you're two hundred fifty yards away.

The interviewer then asks Emily if the sound would be different if the particle was moving toward the source rather than away from it.

Emily: Probably because, you know, the ambulance example, right? Interviewer: What's that?

Emily: So it's the ambulance goes by you, you get that the doppler effect, right?

Interviewer: What is that?

Emily: I don't know. Uhm. So it's far away. You can't hear it as well. It comes toward you, gets really really loud and painful and then it goes away. So if you're standing still that's kind of what happens. And then, I'm not sure. That's all that I know about it. 
Interviewer: Okay, so it's mostly a volume thing that is playing a part?

Emily: Yeah.

Although Emily expresses a great deal of doubt about her description of the Doppler effect, she does incorrectly cite the Doppler effect for the change in intensity rather than frequency.

Whether the students who describe intensity are conflating it with the Doppler effect can not be conclusively determined from the data though. It would require the students explicitly citing the cause of the change in sound intensity. Most students simply stated the cause was due to the particle moving away from the source. That could mean they correctly described loss of intensity due to the spherical wave front or incorrectly due to the Doppler shift.

\subsubsection{Real-World Contextualization}

Students' examples of real-world applications were sorted into the biomedical and nonbiomedical categories (Fig. 7.3). The biomedical category contained responses that described ultrasound imaging and Doppler blood flow monitors. The non-biomedical category included examples like animal echolocation, sonar/radar, and acoustics. This category also contained answers that can be described as standard textbook examples, e.g. an ambulance driving past a stationary observer. Multiple students gave more than one example (Fig. 7.4). For example, one student referenced both echolocation as used by bats and ultrasound imaging. Therefore the same student could be represented in both the medical and non-medical categories. Because of this, the category percentages do not sum to 100 percent. Furthermore, chi-square tests of independence could not be conducted because the categories were not unique. 


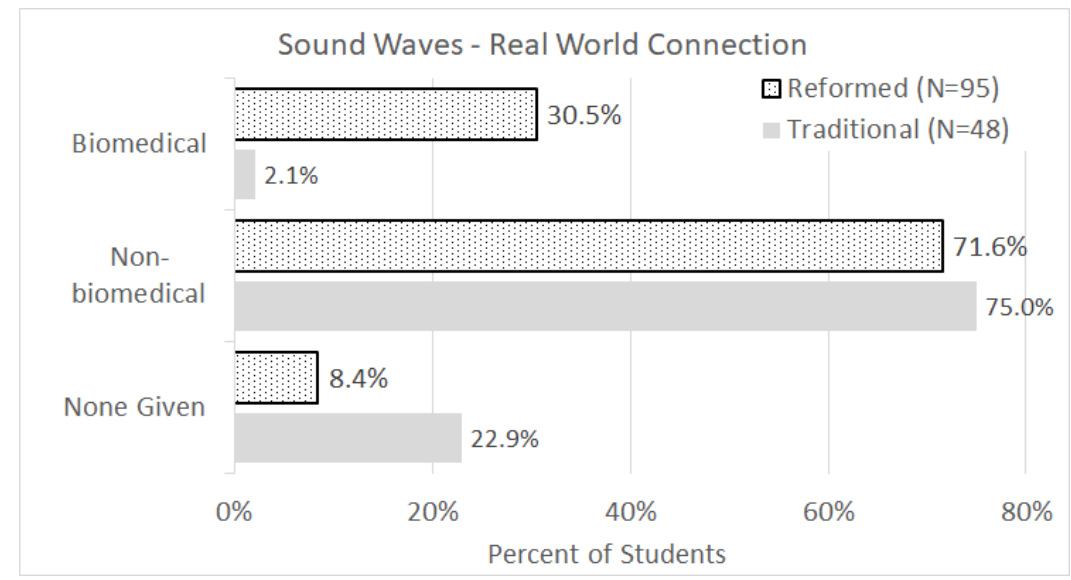

Figure 7.3: Distribution of students' contextualization of sound wave illustrated quiz prompt. Note that multiple students gave responses with more than one example. Therefore the category percentages do not sum to 100 percent.

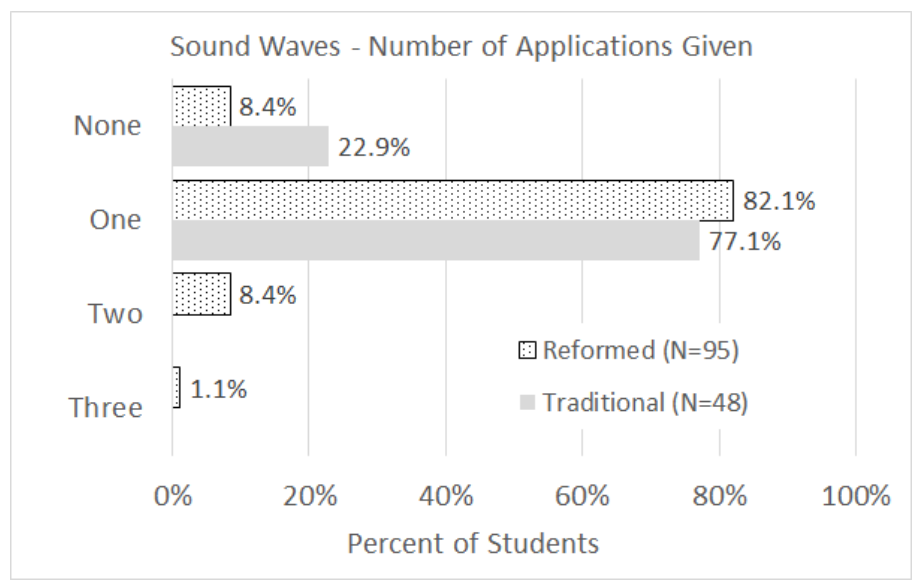

Figure 7.4: Distribution of the number of real-world application of the phenomena depicted in Fig. 7.1.

\subsubsection{Discussion}

The fact that only a portion of the students in both classes described the Doppler effect makes comparisons between cohorts difficult. Although students from the reformed course were more likely to describe the Doppler effect, those who did describe it did so correctly regardless of the course they were in. 
Greater understanding could be gained if students were asked to more clearly defend their response. For example, equations could be required as part of their answer. If students cited the equation that describes the intensity as a function of distance as their reasoning for quieter sound, they would be correct. If however they cite the Doppler equation, they would be incorrect. This might illuminate students' understanding more clearly.

Students in the reformed course clearly showed a greater likelihood of supplying a biomedical example than students in the traditional course. A similar percentage of both groups cited non-biomedical examples. The students in the traditional course were more likely to not cite any real-world applications at all. It remains unclear whether students in either group were more likely to understand concepts like the Doppler effect though, due to the low numbers of students that mention the effect at all.

\subsection{Total Internal Reflection}

\subsubsection{Illustration Prompt}

The illustration on this topic depicted a light ray inside a medium with an index of refraction of $n_{1}$ (Fig. 7.5). The description given to students stated, "The image depicts a light ray interacting with different media." This was followed by the general instructions shown in Fig. 3.7. 
The image depicts a light ray interacting with different media. Using the illustration below:

1. Complete the diagram by drawing the path of the ray as it interacts with the media. Include as much detail as you can including descriptions of the physical principles that predict the path of the ray.

( $0.5 \mathrm{pt}$. for attempting a solution and description $+0.5 \mathrm{pt}$. for a correct solution and description)

2. BONUS: Describe at least one real-world application of this physical principle that is used outside of a physics classroom. Include as much detail as you can including describing how the principle is used.

( $0.25 \mathrm{pt}$. for a correct description of the real world application)

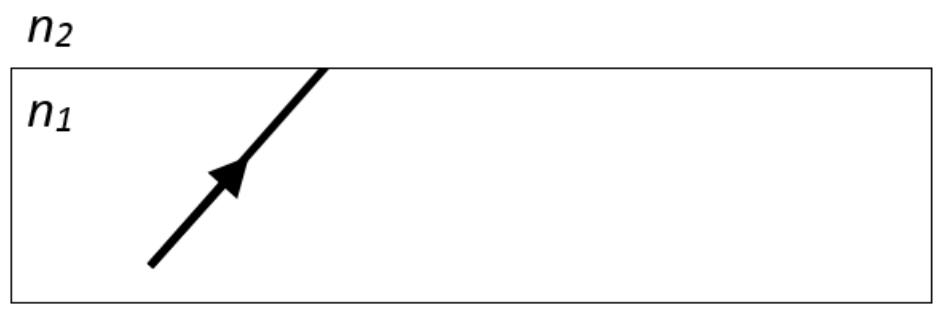

Figure 7.5: Illustration given to students for the quiz on total internal reflection.

The ray is incident on the surface boundary with another medium with an index of refraction of $n_{2}$. The full path of the ray will depend on the refractive indexes and the angle of incidence. One possibility is that the ray will split into two beams. The first ray will refract as it exits the $n_{1}$ medium into the $n_{2}$ medium. The second ray will reflect back into the $n_{1}$ medium. Another possibility is the phenomenon called total internal reflection (TIR). If $n_{1}>n_{2}$ and the angle of incidence is greater than the critical angle, $\theta_{c}=\sin ^{-1}\left(n_{2} / n_{1}\right)$, no light will exit the block. The light ray will reflect off of the top and bottom surfaces of the $n_{1}$ medium, moving down the length of the block. This allows light to be "guided" along the length of the medium. This is the underlying physical concept behind fiber optics and endoscopic surgery.

The focus of the analysis of this quiz was on how students drew the reflection and/or refraction of the ray at the top and bottom surfaces of the $n_{1}$ medium. The possible reflection and refraction events are depicted in Fig. 7.6. 


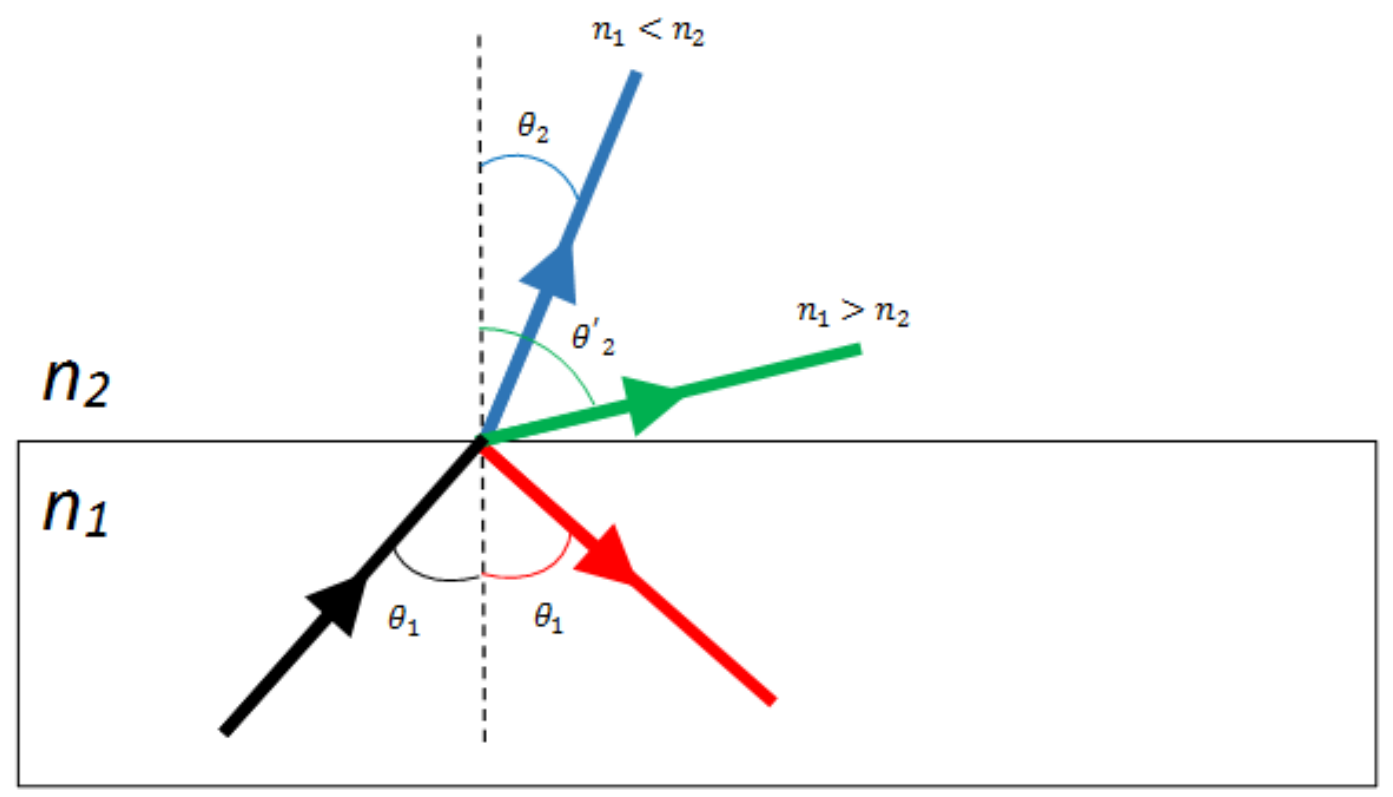

Figure 7.6: Illustration of the possible refraction and reflection events for the prompt in Fig. 7.5. The angle of reflection equals the angle of incidence $\theta_{1}$. Snell's Law states that the refracted ray will either bend toward or away from the normal depending on the values of $n_{1}$ and $n_{2}$. If $n_{1}<n_{2}$ and the incident angle is greater than the critical angle, all of the light will reflect and no light will refract. The angles are taken with respect to the normal.

The law of reflection states that the incident angle is equal to the reflected angle. Reviewers rated students' responses based on whether the reflected ray appeared to have been drawn at an angle other than the incident ray.

The law of refraction, or Snell's Law, states that

$$
n_{1} \sin \theta_{1}=n_{2} \sin \theta_{2},
$$

where $\theta_{1}$ and $\theta_{2}$ are the incident and refraction angles respectively. This means that if $n_{1}>n_{2}$ the refracted ray will bend away from the normal and that if $n_{1}<n_{2}$ the refracted ray will bend toward the normal. Each student was rated on whether they correctly drew the refraction based on the student's rankings of $n_{1}$ and $n_{2}$. 
We also examined the depiction of angles. Both the law of reflection and refraction assume angles with respect to the normal as in Fig. 7.6. Students were rated on whether they drew angles with respect to the normal, the surface boundary between $n_{1}$ and $n_{2}$, or a mixture of both.

\subsubsection{Conceptual Understanding}

All students who submitted a quiz (92 in the reformed and 49 in the traditional course) drew either a refraction, a reflection, or both (Fig 7.7). Students in both groups were more likely to draw a refraction without a reflection. This is incorrect since there is always some amount of reflected light at a surface boundary. Though it is not surprising that a majority of students made this mistake. It is common practice in physics curriculum to illustrate refraction without reflection. This is done to simplify instruction when focusing on refraction. Chi-square tests do not indicate that there is a relationship between this distribution and the course reforms, $\chi^{2}(2, N=141)=$ $5.27, p=0.07$.

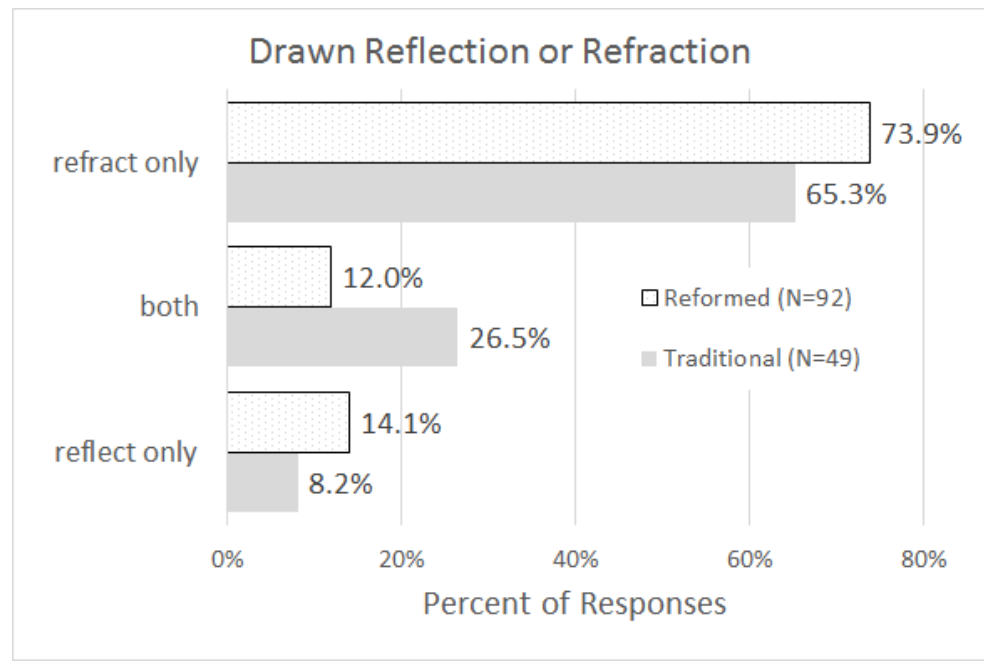

Figure 7.7: Distribution of events drawn by students to the prompt in Fig. 7.5. 
The goal of this quiz was to probe students' understanding of TIR, but only $26 \%$ of the reformed course and $34 \%$ of the traditional course drew reflections. The student interviews may partially reveal why the majority of students drew refraction events without reflections. Of the four students who were given the TIR prompt during the interview, only one drew the complete $n_{1}$ block. The other three students only drew the upper surface boundary. This might suggest that they did not view the $n_{1}$ as being a finite block. One student, who will be called Alan, initially only drew the top surface. Alan suggested TIR only after the interviewer prompted him to think about the bottom surface.

Alan: ...Some of it's also going to reflect and what we see is going to be what it reflects...

Interviewer: Okay so what happens that way, so maybe if you can continue your drawing just a little bit. The reflected ray, what happens to that as it persists? If there's a bottom like in the picture there [indicated the printed version given to the student].

Alan: Like an eye or something?

Interviewer: If we assume...there's $n_{2}$ on both sides of this. So there's $n_{1}$ in the middle and then it kind of reflects back down. What happens when it hits the other surface on the other side?

Alan: Oh, well then you'd be in the ballpark of total internal refraction $[s i c]$, I believe. Where if it was not at the critical angle then it would just all just shoot back and forth ad infinitum like that. Like a fiber optic cable.

Alan demonstrates some misconceptions. He incorrectly names the phenomena as total internal refraction rather than reflection. His analysis of the critical angle is vague. The point though is that he did not recognize the possibility of TIR until the interviewer asked him to consider the bottom surface.

Although few students depicted a reflection, they were highly likely to be drawn 
correctly. 24 students (26.1\%) from the reformed course and 17 students (34.7\%) from the traditional course drew a reflection at either the top or bottom surface of the $n_{1}$ medium. Of those 41 students, only two drawings were judged by the reviewers as being incorrect. Therefore there was no significant difference between cohorts.

Students' drawings of refraction events at the top surface showed a greater diversity of answers (Fig. 7.8). Raters categorized a drawing based on whether the student showed the correct direction of the refraction. In order to correctly judge the refraction, the student had to specify the refractive indexes of the two materials in their answer. If the student did not indicate values, they were categorized as 'n not specified'.

Students in the traditional course were more likely to draw the correct refraction, however the chi-square test for independence did not indicate that there was a relationship between this distribution and the course reforms, $\chi^{2}(3, N=124)=5.86, p=$ 0.12. No student drew a refraction event at the bottom surface of the $n_{1}$ block.

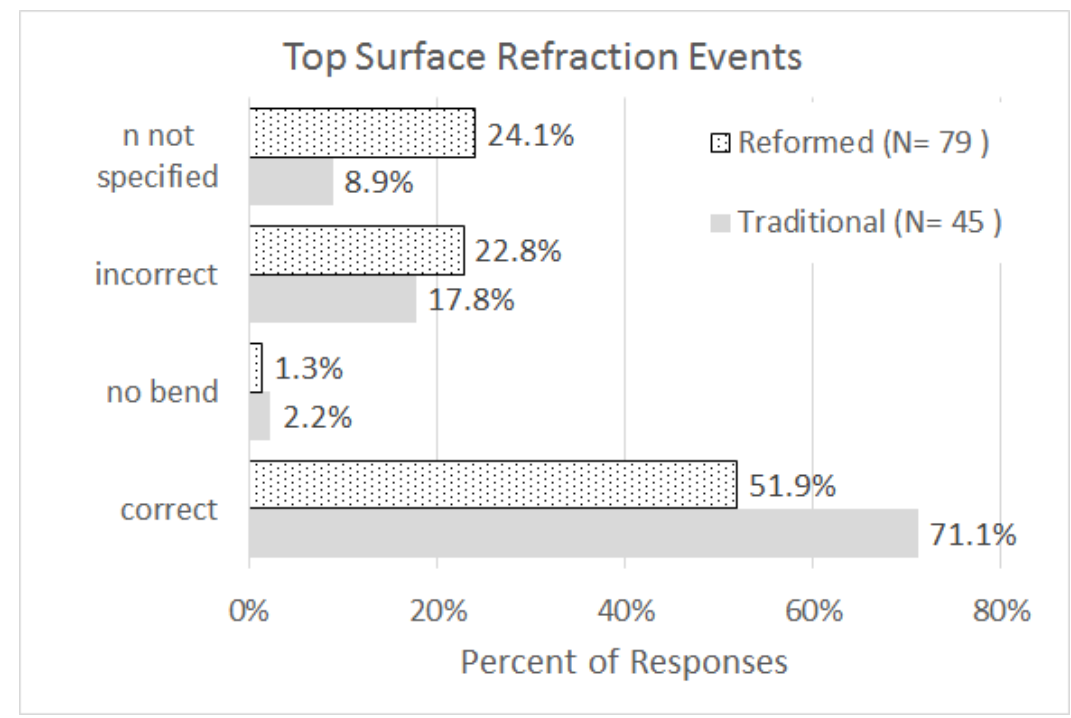

Figure 7.8: Distribution of refraction events drawn by students to the prompt in Fig. 7.5. 
Unlike the previous qualities under investigation, analysis of whether students correctly represented the angles of reflection and refraction was conducted by only a single reviewer. While the original two reviewers did analyze this topic, it was later decided that the categories were not sufficiently comprehensive to describe the variety of responses.

There was a clear difference in the locations of the angles in students' drawings. Students in the traditional course were more likely to correctly draw the angle of reflection and refraction with respect to the normal (Fig. 7.9). Students in the reformed course were more likely to draw the angles with respect to the surface boundary between the $n_{1}$ and $n_{2}$ mediums. Many students drew only the normal line without drawing any angle markings. Other students drew some angle markers with respect to the normal and other markers with respect to the surface. Three students in the reformed course drew angles with respect to the path the ray would take in the absence of the $n_{2}$ material. These responses are represented in the category 'incident' in Fig. 7.9. This error could be a misunderstanding of the word normal, which could have been interpreted by these students as meaning the 'normal path' of the ray in the absence of the $n_{2}$ medium. The chi-square test indicates that there is a relationship between the course type and the location of the drawn angles, $\chi^{2}(4, N=116)=10.38, p=0.03$. 


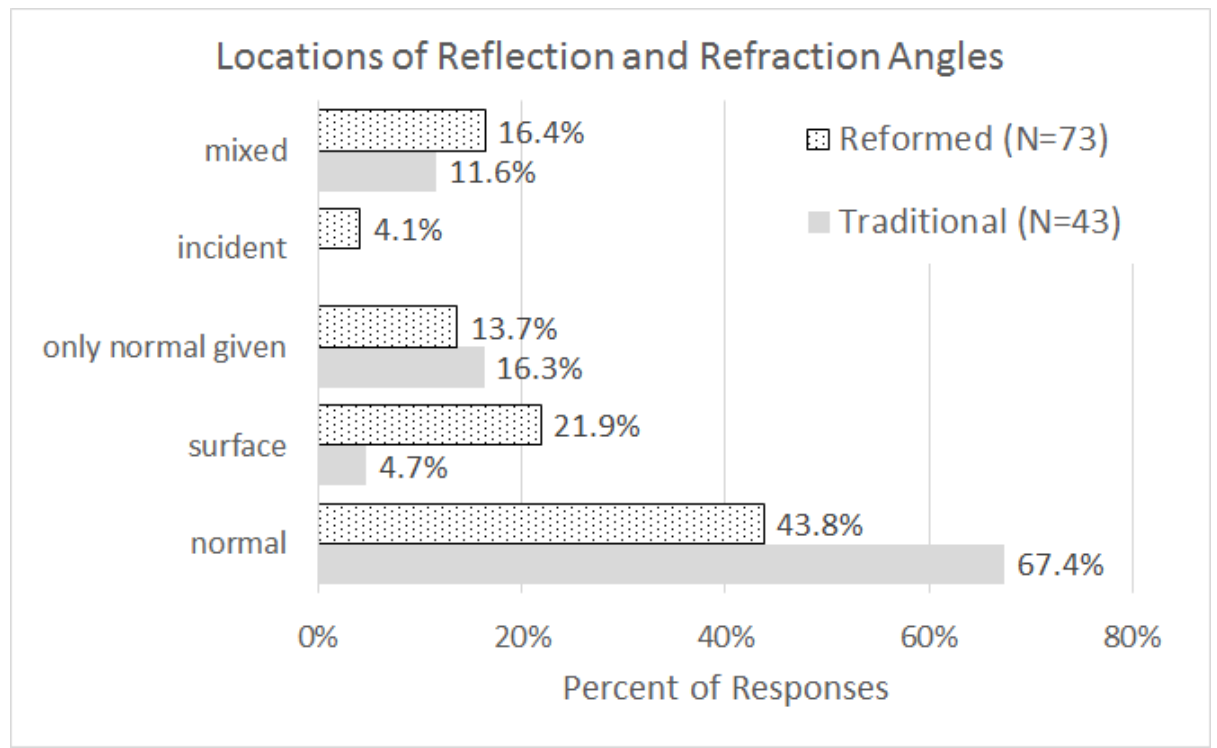

Figure 7.9: Distribution of the angles drawn by students to the prompt in Fig. 7.5. The category 'only normal given' represents drawings where the normal was included but no indication of the location of the angle. The 'incident' represents angles with respect to the path of the incident ray in the absence of the $n_{2}$ medium.

The incorrect responses concerning the refraction at the top surface (Fig. 7.8) can be partially explained though comparison with the angle data. Of the 26 students in both the traditional and reformed courses who drew the incorrect refraction at the top surface, nine either only drew the normal or no angle information, nine correctly drew the angles with respect to the normal, and eight drew angles at least one angle with respect to the surface. Positioning the angles with respect to the surface can lead to incorrect conclusions even when the correct analysis of Snell's Law is followed.

This reasoning was seen during one of the student interviews. The student, who will be called Cathy, drew both the incident and refracted angles with respect to the surface. Cathy was asked to explain why the refracted light bent the way she had drawn it. Cathy correctly wrote Snell's Law on the board and explained her reasoning out loud. She had already drawn the incident angle $\theta_{1}$ with respect to the surface. Text in italics was added to clarify the meaning. 
Interviewer: So if $n_{2}$ is bigger than $n_{1}$, what does that imply?

Cathy: I have to do the math to tell you. [writes Snell's Law in the $\left.\overline{\text { form } \theta_{2}}=\sin ^{-1}\left(n_{1} \sin \theta_{1} / n_{2}\right)\right]$ Okay, so if $n_{2}$ is larger than $n_{1}$, that's going to make this value $\left[\theta_{2}\right]$ smaller? Yeah, smaller.

Interviewer: Okay and why does it make it smaller?

Cathy: Because $n_{2} \ldots$ I guess it's just larger than $n_{1}$. So it's just like two over one versus one over two. So then if $n_{2}$ is larger, that's going to make $\theta_{2}$ smaller.

Interviewer: So can you sketch now $\theta_{2}$ on your picture?

Cathy: I honestly, I think it's right here, $\theta_{2}$. [draws $\theta_{2}$ with respect to the surface] I think. Not entirely sure.

Interviewer: Alright. Is that consistent with what you said? Like the angle gets smaller?

Cathy: Yeah, because this angle $\left[\theta_{2}\right]$ will be smaller than that angle $\left[\theta_{1}\right]$. Because if they're the same then this will be a straight line. So if it's smaller, it's going to be closer to this [the surface].

Using Snell's Law, Cathy correctly stated that if $n_{2}>n_{1}$, then $\theta_{2}<\theta_{1}$. But because she placed both $\theta_{1}$ and $\theta_{2}$ with respect to the surface, she drew the refracted ray bending away from the normal rather than toward it.

\subsubsection{Real-World Contextualization}

Figure 7.10 shows the distribution of the real-world applications of the physical concept illustrated by the prompt in Fig. 7.5. Once again, the students in the reformed course were much more likely to cite biomedical examples than the students in the traditional course. These biomedical examples mostly dealt with endoscopic surgery and fiber optic cables. The non-biomedical examples often referred to responses that identified the $n_{1}$ block as water and the $n_{2}$ block as air. Typical examples of real-world applications from this category include spear fishing, swimming pools, and coins in a 
cup of water. The later example is a common physics education demonstration done in class that shows that the location of a coin in water appears to be in a different location then it actually is due to refraction.

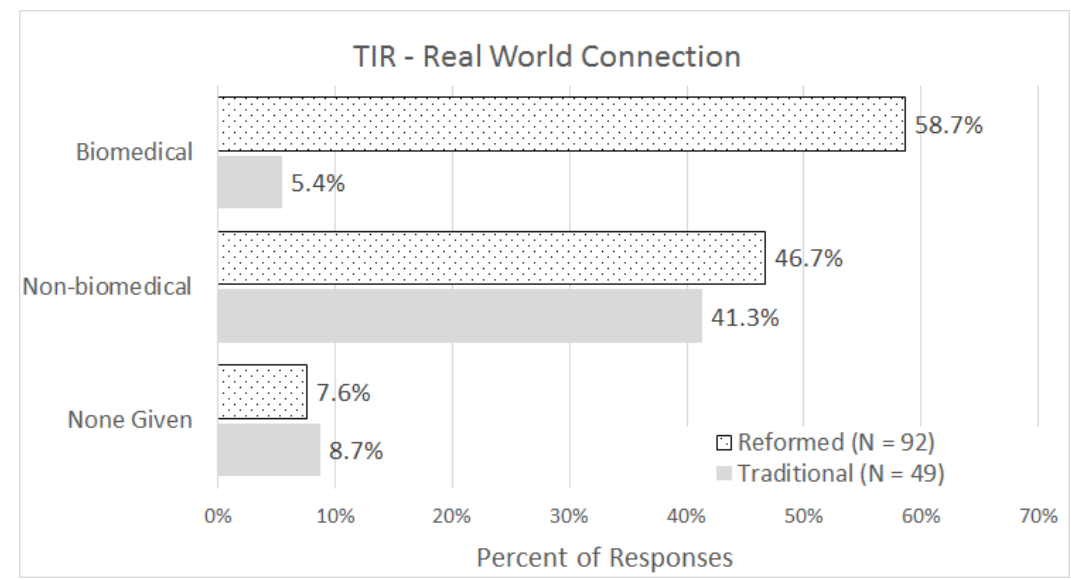

Figure 7.10: Distribution of students' real-world applications of the physical concept depicted in Fig. 7.5. Many students gave multiple examples, so the percentages do not sum to 100 percent.

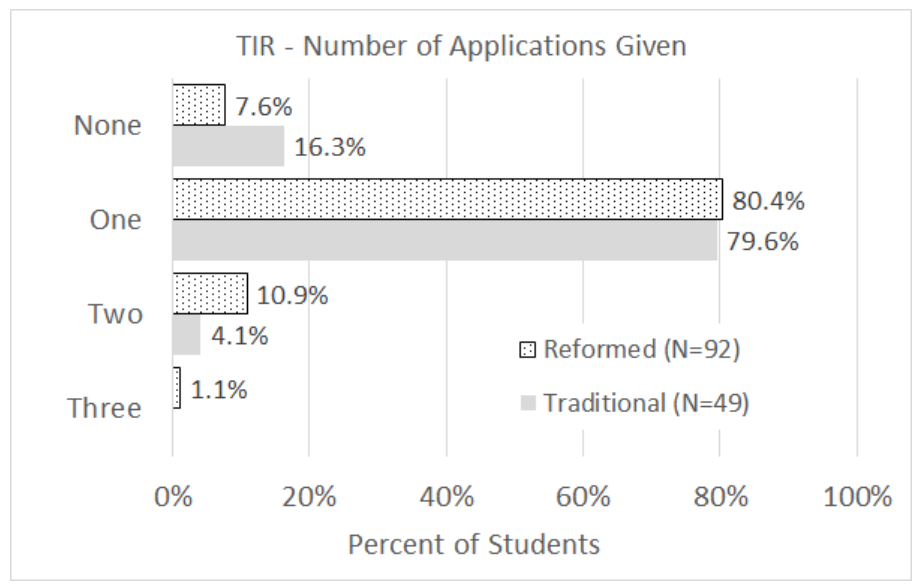

Figure 7.11: Distribution of the number of real-world application of the phenomena depicted in Fig. 7.5.

The reason for these differences could be explained by the focus of the instruction the students received.

Endoscopy was heavily discussed in the reformed course but not in the traditional 
course. Students in the reformed course did an entire laboratory session on endoscopic surgery as well. This activity was not part of the lab curriculum for the students in the traditional course.

Both the traditional and reformed courses had instruction relevant to the category of 'water system'. The students in the reformed course were given an in-class example of the view from the beach of a submerged beach ball. There was also a clicker question that accompanied the demonstration of a coin in a cup. The same demonstration was

performed in the traditional course. Students in the reformed course were walked through an in-class sample problem of a kingfisher in flight locating the true location of a fish underwater. The traditional course also had at least two homework questions on this same phenomenon.

It is worth noting that while 51 students cited either fiber optics or endoscopic surgery, only 15 of those same students drew reflection events. An even smaller number drew TIR. These students still recognized the relevance of refraction and reflection to fiber optics and endoscopy.

\subsubsection{Discussion}

There is clear evidence that the reformed curricula had an impact on students' contextualization of the physical phenomenon, but the extent to which that aided or hindered their conceptual understanding is unclear. While there is some reason to suspect that the students in the reformed course had a slightly poorer conceptual understanding than students in the traditional course, confounding factors due to the open-ended nature of the quiz makes it difficult to make conclusive statements.

The values of the refractive indexes were purposely left blank rather than influence 
students' contextualization of the prompt. If, for example, students were told that the $n_{1}$ material was plastic and the $n_{2}$ material was air, students would probably have been less likely to suggest water based systems. The drawback to not identifying the refractive indexes is that many students did not specify a material. Without knowledge of the relative values of the indexes, reviewers could not rate whether the correct refraction was drawn. Since the students in the reformed course were more likely to omit the refractive indexes, we cannot state conclusively that the reformed curriculum impacted students' understanding of refraction.

Students in the reformed course were significantly more likely to incorrectly draw the angles of reflection or refraction with respect to the surface rather than the normal. The majority of students in both courses included angle markings somewhere in their drawings. This suggests that proper use of angles may not have been emphasized to the same extent as it was in the traditional course.

It can not be stated that students' understanding of TIR was adequately assessed since a majority of students interpreted the prompt as being only a refraction phenomenon. Based on the student interviews, this could be improved by explicitly stating that the $n_{1}$ material is surrounded by the $n_{2}$ material.

Correlations between students' conceptual understanding and real-world applications could not be adequately performed due to the open-ended quality of the quizzes. Many students supplied more than one real-world application. There was a variety of physical concepts that students represented. Any attempts to compare these two qualities result in low sample sizes which may not be representative of either cohort.

Based on the analysis in this section, the following alterations should be made to future implementations of this quiz prompt: 
1. Without giving specific values or naming the materials, the prompt should explicitly state that $n_{1}>n_{2}$.

2. The prompt should state that the $n_{1}$ material is surrounded by the $n_{2}$ material. The illustration should also include $n_{2}$ labels above and below the $n_{1}$ material.

3. Students should be told to include all angle markers. Researchers should simply say 'all angles' rather than 'angles of reflection and/or refraction' as that may be too leading.

4. Students should be told to include only one real-world application.

These changes should simplify the analysis while still allowing for a sufficiently openended quiz that does not artificially lead students to specific conclusions.

\subsection{Thin-Film Interference}

\subsubsection{Illustration Prompt}

The illustration for this topic is displayed in Fig. 7.12. It shows a light ray incident on the surface boundary between two mediums with refractive indexes $n_{1}$ and $n_{2}$. Below the $n_{2}$ layer is a third layer with a refractive index of $n_{3}$. The description given to students stated, "The image depicts a light ray interacting with different media." This was followed by the general instructions shown in Fig. 3.7. 
The image depicts a light ray interacting with different media. Using the illustration below:

1. Complete the diagram by drawing the path of the ray as it interacts with the media. Include as much detail as you can including descriptions of the physical principles that predict the path of the ray.

( $0.5 \mathrm{pt}$. for attempting a solution and description $+0.5 \mathrm{pt}$. for a correct solution and description)

2. BONUS: Describe at least one real-world application of this physical principle that is used outside of a physics classroom. Include as much detail as you can including describing how the principle is used.

( $0.25 \mathrm{pt}$. for a correct description of the real world application)

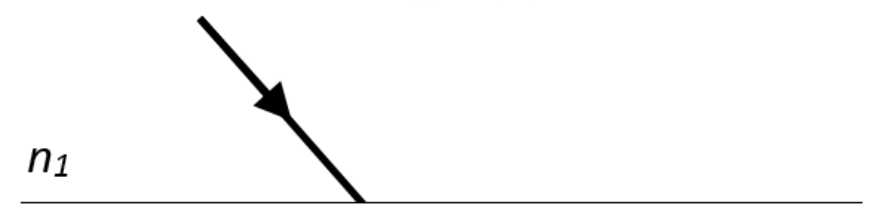

$n_{2}$

$n_{3}$

Figure 7.12: Illustration given to students for the quiz on thin-film interference.

Thin-film interference describes a phenomenon where light reflected from the top and bottom surfaces of the film interfere and either increase or decrease the intensity of the light reflected off of the top surface. Whether the light constructively or destructively interferes depends on the wavelength of the light, the refractive indexes of the mediums, and the thickness of the film $d$. The film thickness is important because the light that has reflected off the bottom surface of the film has traveled farther than the light reflected off of the top surface. The path-length difference between the two rays is $2 d$. Another central concept to this phenomenon is that light that reflects off of a medium high a higher refractive index than the original medium will undergo a $180^{\circ}$ phase shift. No phase shift will occur when reflected off a medium with a lower refractive index or during refraction. This alters the pathlength difference by a half of a wavelength.

Figure 7.13 shows the customary way of depicting thin-film interference. The incident ray will both reflect and refract at the $n_{1} / n_{2}$ surface. The refracted ray 
will also reflect off the $n_{3}$ surface. This ray will either constructively or destructively interfere with the ray reflected at the $n_{1} / n_{2}$ surface. The angles of reflection and refraction are taken with respect to the normal. Reflections and refractions are only shown at the first surface of Fig. 7.13, but occur at each interface.

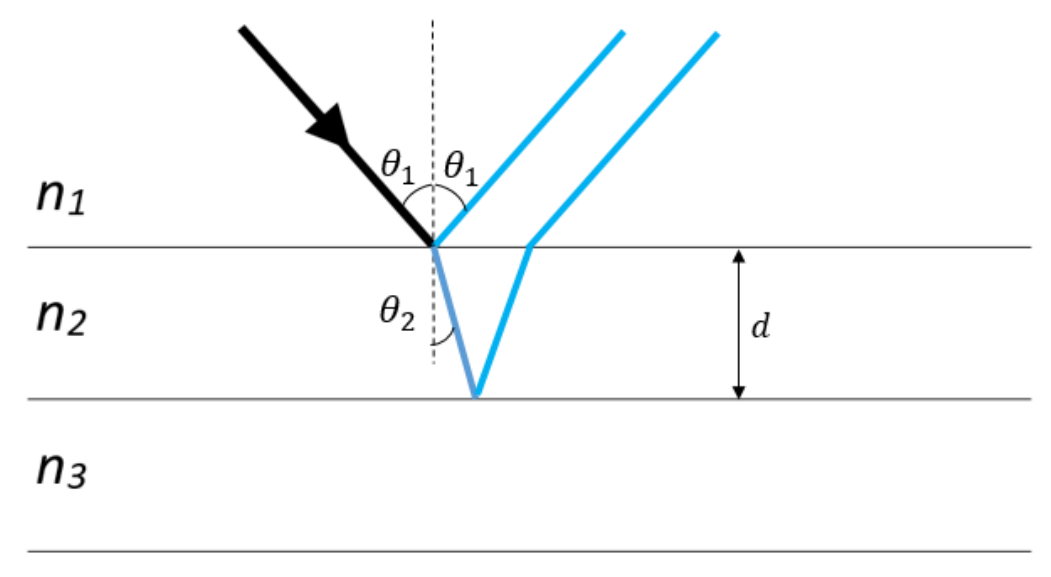

Figure 7.13: Customary depiction of thin-film interference.

The type of interference will depend on the wavelength of the light, the refractive indexes of all three mediums, and the thickness of the $n_{2}$ layer. There are three common ways of depicting thin-film interference. A common situation where $n_{1}<$ $n_{3}<n_{2}$ is a layer of oil on water. Soap films are often used to describe situations where $n_{1}=n_{3}<n_{2}$. In both of these cases, there is a phase change at the top surface but not at the second surface. The conditions for constructive and destructive interference are given by

$$
\begin{array}{r}
2 d=\frac{m \lambda}{n_{2}} \quad \text { (destructive) } \\
2 d=\left(m+\frac{1}{2}\right) \frac{\lambda}{n_{2}} \quad \text { (constructive) }
\end{array}
$$

where $m=0,1,2, \ldots$ 
The third example is when $n_{1}<n_{2}<n_{3}$. Anti-reflective coatings are an example of this situation. In this case, there would be a phase change at each side of the $n_{2}$ medium. This means that the only phase change is due to thickness of the film.

\subsubsection{Conceptual Understanding}

Refractive Index Rankings One indicator of how students interpreted the illustration prompt is how they defined the three refractive indexes. Coding of these relationships was based on explicit numerical values (e.g. $n_{1}=1.0, n_{2}=1.5, n_{3}=$ 1.33), inequalities (e.g. $n_{1}<n_{3}<n_{2}$ ), or written descriptions (e.g. $n_{1}$ is air, $n_{2}$ is oil, $n_{3}$ is water). Figure 7.14 show the distribution of students' description of the different refractive indexes. Each of the categories are described below. There was not a significant relationship to the course reforms, $\chi^{2}(3, N=104)=3.78, p=0.29$.

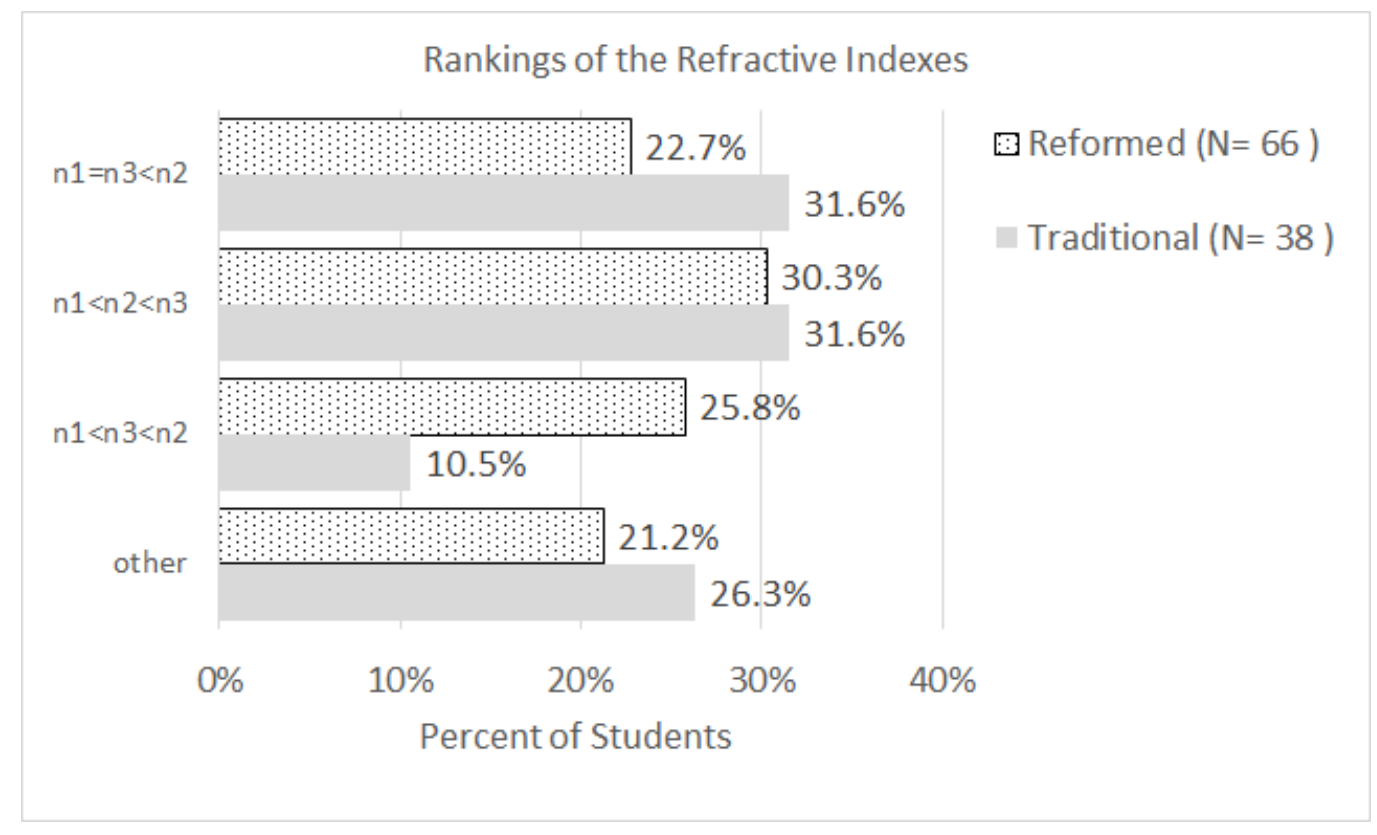

Figure 7.14: Distribution of students' rankings of the refractive indexes for Fig. 7.12.

Many students did not provide sufficient information on the relative values of the 
refractive indexes. This is a similar problem as that experience in the analysis of the total internal reflection prompt in Sec. 7.2. An additional problem in the thin-film interference quizzes was that only part of the relationship between the three refractive indexes was provided. Student in the 'other' category often failed to provide complete information. Many of these students defined the relationship between $n_{1}$ and $n_{2}$, but gave no information at all about $n_{3}$. Other students described the relationship between $n_{1}$ and $n_{2}$ as well as between $n_{2}$ and $n_{1}$, but failed to define the relationship between $n_{1}$ and $n_{3}$.

The relative rankings of the indexes of refraction are not necessarily direct evidence of thin-film interference responses. In a few cases students specified one of these three situations, but only drew refractions. Rather than thin-film interference, these students often described more general refraction phenomena such as light passing through a pane of glass or the cornea of the eye.

It should be noted that while two student researchers coded the rankings of the refractive indexes, I later re-coded some of these responses. The initial coding grouped responses that fit in the ' $n_{1}<n_{3}<n_{2}$ ' under the 'other' category'. Using the new category, I re-coded the quizzes that were originally placed in the 'other' category. The other two categories are the original analysis by the student researchers.

Phase Shifts Only a fraction of the students in both courses described phase shifts upon reflection. The vast majority of those students correctly stated that a phase shift would occur when the light reflects off a medium with a higher index of refraction than the incident medium. Students' description of any phase shift at either the $n_{1} / n_{2}$ or $n_{2} / n_{3}$ surface was analyzed. The results are presented in Table 7.1. 
Table 7.1: Students descriptions of the phase shift of the light ray in Fig. 7.12.

\begin{tabular}{l|l|l|l}
\multicolumn{2}{c|}{ Surface Boundary } & Reformed & Traditional \\
\hline \hline & Correct & 24 & 21 \\
$n_{1} / n_{2}$ & Incorrect & 1 & 2 \\
\hline & Correct & 12 & 12 \\
$n_{2} / n_{3}$ & Incorrect & 0 & 1 \\
\hline
\end{tabular}

Reflection and Refraction Although reflection and refraction were not the primary features under analysis, the coding scheme from the total internal reflection quiz (Sec: 7.2) was directly applicable to the quizzes about thin-film interference.

The analysis explored how students depicted reflection and refraction at three locations called the first, second, and third surfaces. These are represented in Fig. 7.15. The first and second surfaces are the points where the light ray passes through the $n_{1} / n_{2}$ and $n_{2} / n_{3}$ boundaries respectively. The third surface is the point where light that has reflected off the $n_{3}$ medium passes back into the $n_{1}$ medium.

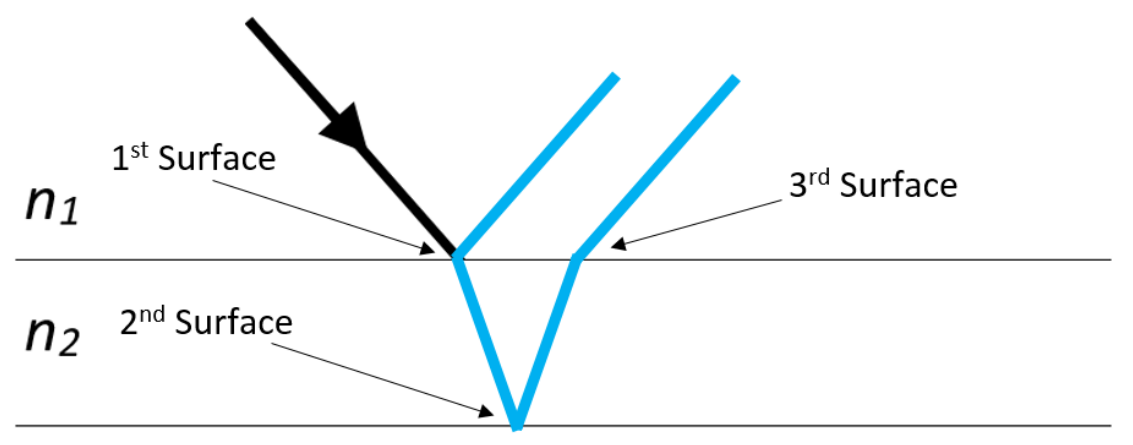

\section{$n_{3}$}

Figure 7.15: Locations of the surfaces used in the analysis of reflection and refraction events for Fig. 7.12.

The majority of students in both courses drew reflection events at both the first and second surfaces. Almost all of these students' drawings were as accurately depicting 
the law of reflection. Very few students drew a reflection at the third surface. This result is to be expected given students' success at depicting the same principle in the TIR drawings.

The types of refraction events drawn by students at each of the three surfaces are shown in Fig. 7.16. The categories are the same as those used in the analysis of the TIR quiz (Sec. 7.2). These quizzes suffered from the same problem of students not specifying the relationships between the refractive indexes. It is therefore not possible to determine the accuracy of refractions drawn by those students.

As shown in Fig. 7.16(a), nearly every student who submitted a quiz drew light passing through the first surface. Students in the traditional course were more likely to draw no refraction at the first surface, but rather draw a straight line that continues from the incident, printed light ray. This is shown in the 'no bend' category. The refraction events at the first surface were not independent of the course reforms, $\chi^{2}(5, N=141)=16.96, p<0.005$. 


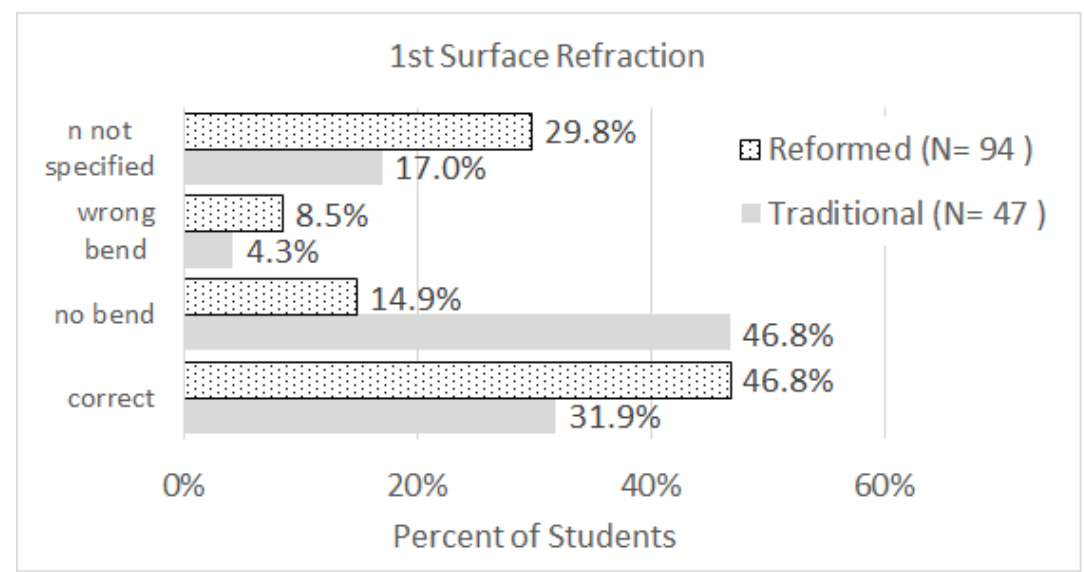

(a)

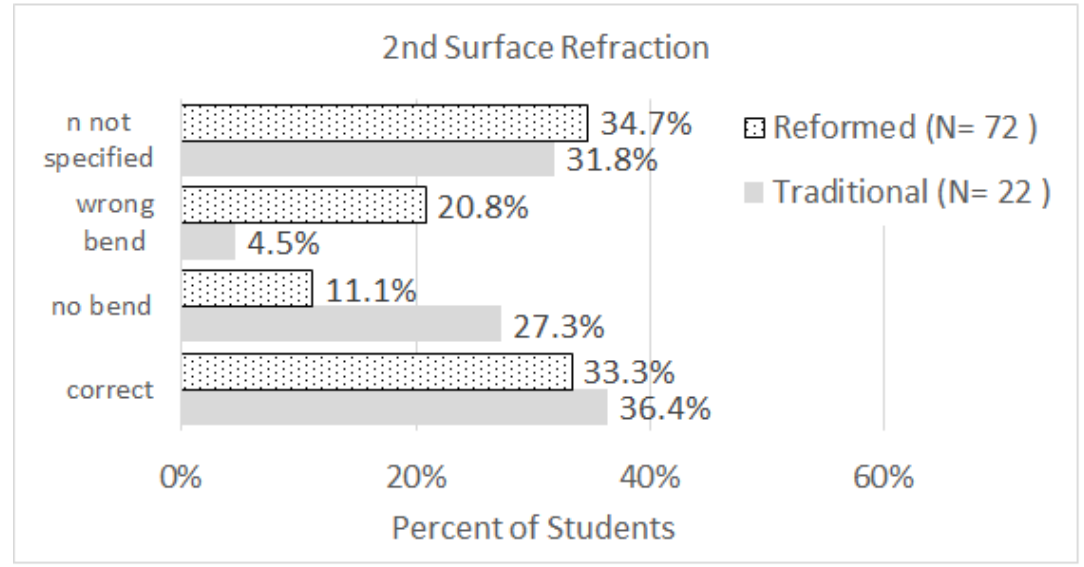

(b)

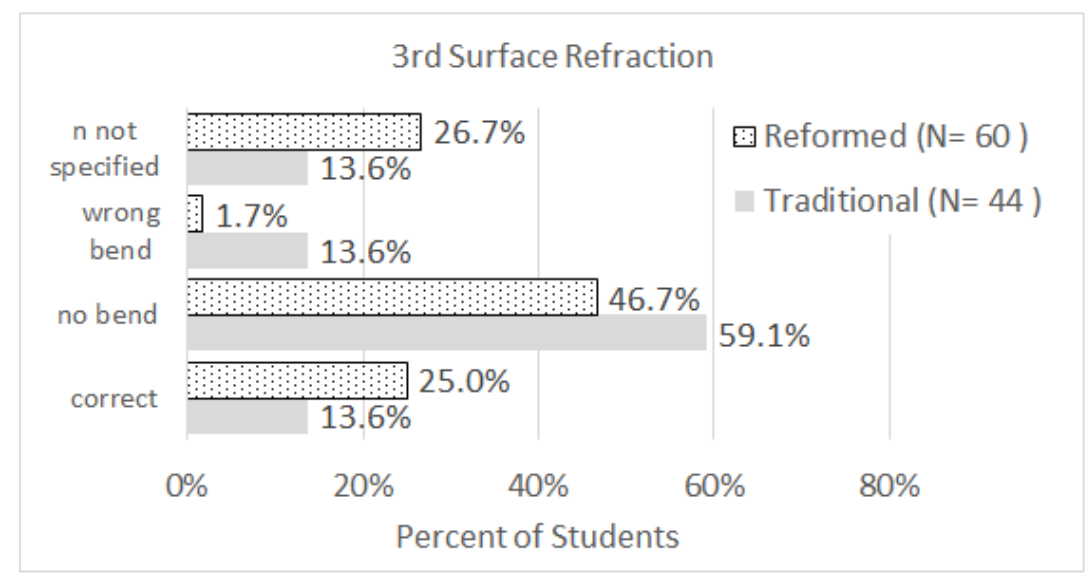

(c)

Figure 7.16: Distribution of students' depictions of refraction events for Fig. 7.12. The location of the three surfaces are shown in Fig. 7.15. 
Students in the traditional course were more likely than the students in the reformed course to draw no refraction at the second surface as well. However, more students in the reformed course were more likely to draw a refraction that bends in the wrong direction. Despite these differences, there was not a significant relationship between the refractions at the second surface and the course reforms, $\chi^{2}(5, N=94)=5.67, p=0.34$.

A high proportion of students in both courses drew no refraction at the third surface. There was not a significant relationship between the refractions at the third surface and the course reforms, $\chi^{2}(5, N=104)=9.82, p=0.08$.

It is interesting that such a large number of students failed to draw a refraction ('no bend' category) as the light ray passed through two different mediums. A conclusive statement about the cause of this cannot be made based on the data in the quizzes. However, it is worth noting that this issue was not prevalent in the TIR quiz, which also rated refraction events. (See Fig. 7.8.) Fourteen students from the reformed course and 22 from the traditional course failed to draw a refraction at the first surface of the thin-film interference quiz. Figure 7.17 lists the types of refractions those same students gave to the TIR quiz. A large number of the students who failed to draw a refraction in the thin-film interference quiz, correctly drew refractions in the TIR quiz. 


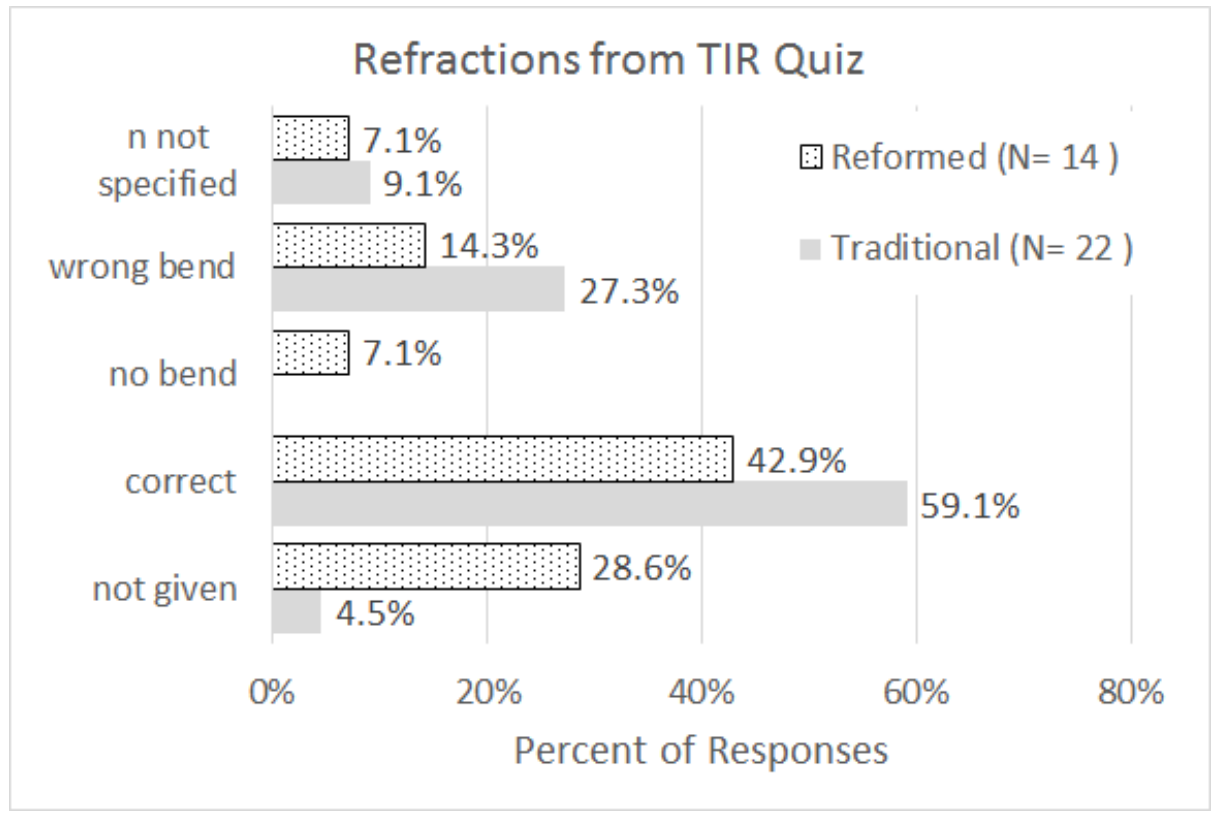

Figure 7.17: Distribution of students' depictions of refraction events from the TIR quiz (Sec. 7.2). Only students from the 'no bend' category from Fig. 7.16(a) are included here.

Depictions of Angles Students were less likely to include angle markers than they were in the TIR quiz (Fig. 7.9). Only five students from the traditional course indicated the position of either the angle of refraction or reflection. All but one of these students correctly drew the angles with respect to the normal. Forty students from the reformed course drew angle markers. Sixteen of these students incorrectly drew at least one marker with respect to the surface. This was also a problem for the students in the reformed course who completed the TIR quiz.

\subsubsection{Real-World Contextualization}

The distribution of students' real-world applications is shown in Fig. 7.18. Typical examples from the biomedical category include microscopes and human vision. The non-biomedical examples focused on the examples cited previously in this section: 
soap bubble, oil slicks, and anti-reflection coatings. The non-biomedical examples also included a number of purely refraction events, e.g. light traveling through a pane of glass.

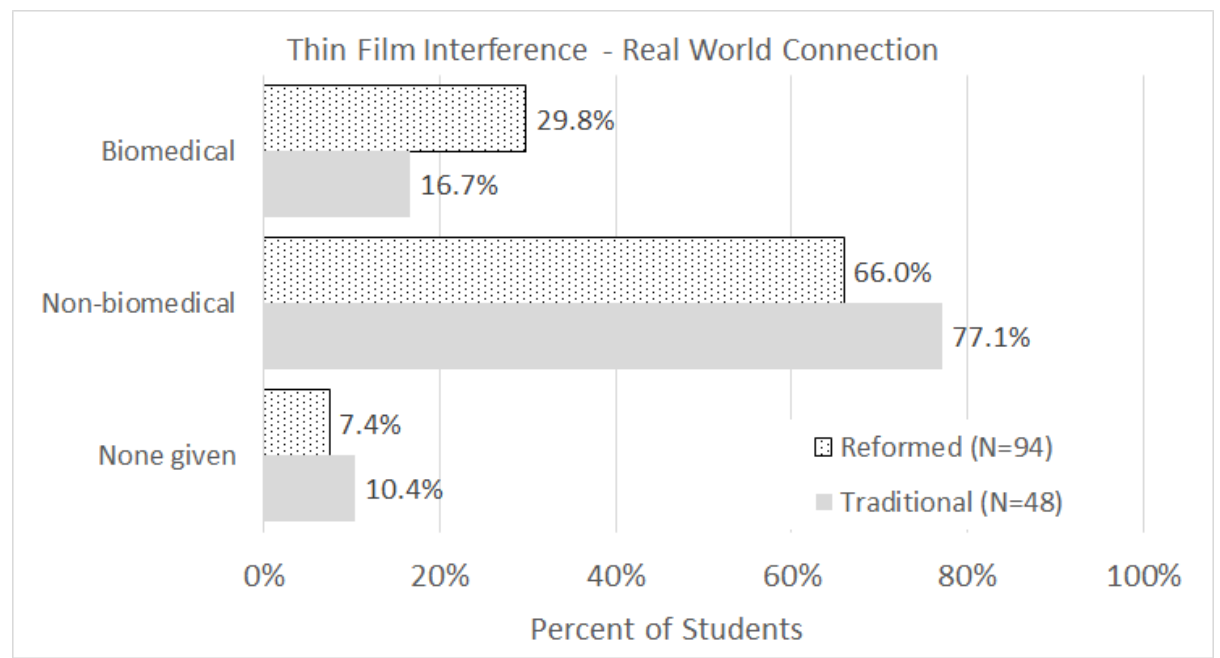

Figure 7.18: Distribution of students' real-world applications of the concept depicted in Fig. 7.5.

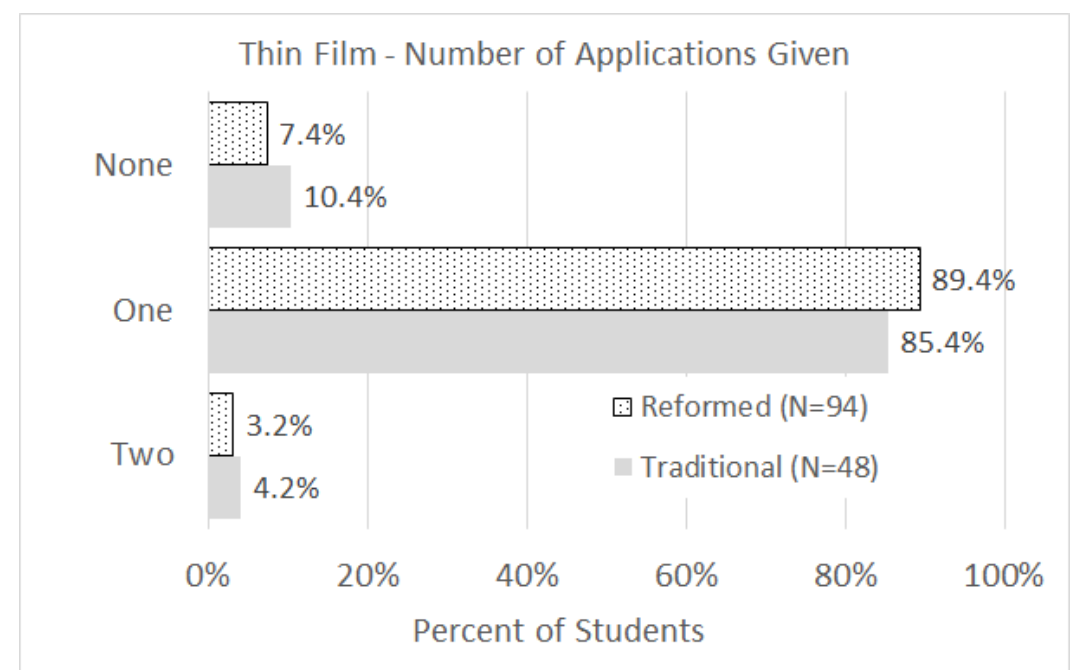

Figure 7.19: Distribution of the number of real-world applications of the concept depicted in Fig. 7.5.

Compared to the TIR and sound waves quizzes, the difference in the number of biomedical examples between courses is much smaller. One cause of this is that micro- 
scopes were prevalent among both the reformed and traditional groups. Microscopes were counted as biomedical because they were the focus of an entire module of the reformed course. They were also discussed in the traditional course, though to a much smaller extent and without a strong focus on their use in medicine. Another issue is that compared to the sound waves and TIR, thin film interference was not as strongly emphasized in a biomedical context. The use of thin films in biomedicine only constituted a small portion of the microscopy module. Students in the reformed course may have had more exposure to thin films using the more traditional applications.

\subsubsection{Discussion}

No significant differences could be found in students' conceptual understanding of the prompt. However the open-ended nature of the illustration allowed for a large variety of responses and therefore greatly limited the analysis. Students understanding of thin-film interference specifically could not be conclusively determined since so many students either failed to mention it. Furthermore, those who did describe it, gave only general descriptions.

Analysis of this quiz was complicated by the fact that so many students from both groups failed to define the relationship between the refractive index of each material. Defining the relative values though would possibly suggest a real-world application for students. Students would be less likely to cite soap films if the refractive indexes given by the prompt suggest an oil slick. Future versions of this prompt should compel students to define the refractive index of each material, either with explicit numbers or with inequalities.

Without specifying a thickness of the $n_{2}$ material or the frequency of the incident light, it is difficult to know whether students can correctly determine whether the 
light reflected off of the first surface is constructively or destructively interfering with light reflected off the second surface. Defining the frequency of light would probably have little influence on how students perceive the prompt. Although citing a thickness could influence their responses. Future versions should therefore define the frequency of light, but only include a symbol $d$ for the thickness. Students should be explicitly prompted to define that thickness for themselves.

Students' real-world applications for the thin film interference prompt noticeably differed from those for the sound waves and TIR illustrations. In those quizzes, students were much more likely to cite biomedical examples. While students in the reformed course were more likely to cite biomedical examples than students in the traditional course, the split was much smaller. This was quite possibly because the focus of the instruction for thin-films did not put the same emphasis on medicine that the previous topics did.

\subsection{Image Formation by a Lens}

\subsubsection{Illustration Prompt}

The illustration for this topic (Fig. 7.20) depicts light rays from a point source incident on a convex lens. The description given to students stated, "The image depicts light rays interacting with different media." This was followed by the general instructions shown in Fig. 3.7. 
The image depicts light rays interacting with different media. Using the illustration below:

1. Complete the diagram by drawing the path of the rays as they interact with the media. Include as much detail as you can including descriptions of the physical principles that predict the path of the rays.

( 0.5 pt. for attempting a solution and description $+0.5 \mathrm{pt}$. for a correct solution and description)

2. BONUS: Describe at least one real-world application of this physical principle that is used outside of a physics classroom. Include as much detail as you can including describing how the principle is used.

( $0.25 \mathrm{pt}$. for a correct description of the real world application)

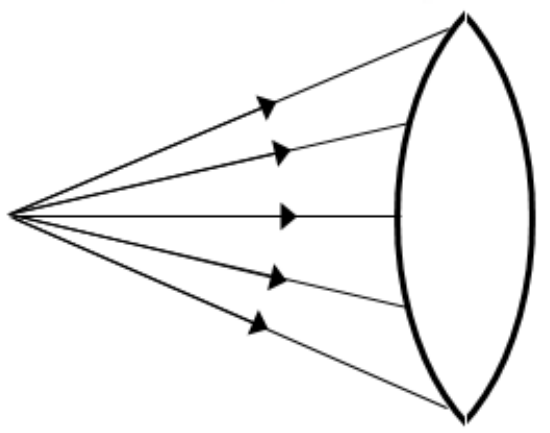

Figure 7.20: Illustration given to students for the quiz on image formation by lenses.

The behavior of the rays as they exit the lens will depend on the distance between the lens and the point source. If the point source is located at the focal point of the lens, the light rays will exit the lens parallel to the optical axis (Fig. 7.21(a)). If this distance is greater than the focal length of the lens, the rays will converge (Fig. 7.21(b). If the light source is located between the focal point and the lens, the exiting rays will diverge (Fig. $7.21(\mathrm{c})$ ). 


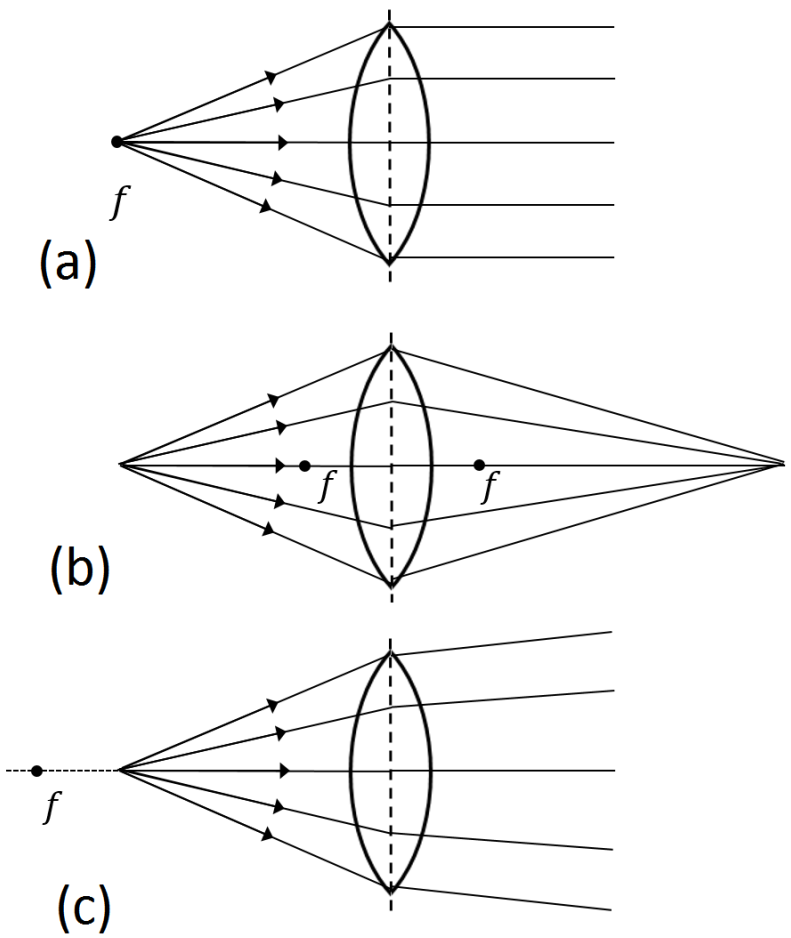

Figure 7.21: Possible correct answers to the prompt in Fig. 7.20. The paths of the rays exiting the lens depend of the location of the point source to the focal point $(f)$. (a) If the point source is located at the focal point, the rays will exit parallel to the optical axis. (b) If the point source is located farther than the focal point, the rays will converge. (c) If the source is located in between the focal point and the lens, the rays will diverge. Note that each image uses a different scale.

\subsubsection{Conceptual Understanding}

Almost all of the students described the object in Fig. 7.20 as a lens. One student in the reformed course and three students in the traditional course interpreted the object as being a mirror. These four students were removed from further analysis of conceptual understanding. With this subtraction, a total of 90 students from the reformed course and 45 from the traditional course submitted a quiz.

In order to assess whether students correctly drew the paths of the rays, the focal point of the lens must be determined. However, focal points were drawn or described 
by only $38.9 \%(\mathrm{~N}=35)$ of students from the reformed course and $53.3 \%(\mathrm{~N}=24)$ of students from the traditional course. Figure 7.22 shows the distribution how students drew the focal point of the lens. There is not a statistically significant relationship between the location of the focal points as drawn or described by students and the course reforms, $\chi^{2}(2, N=59)=3.86, p=0.15$.

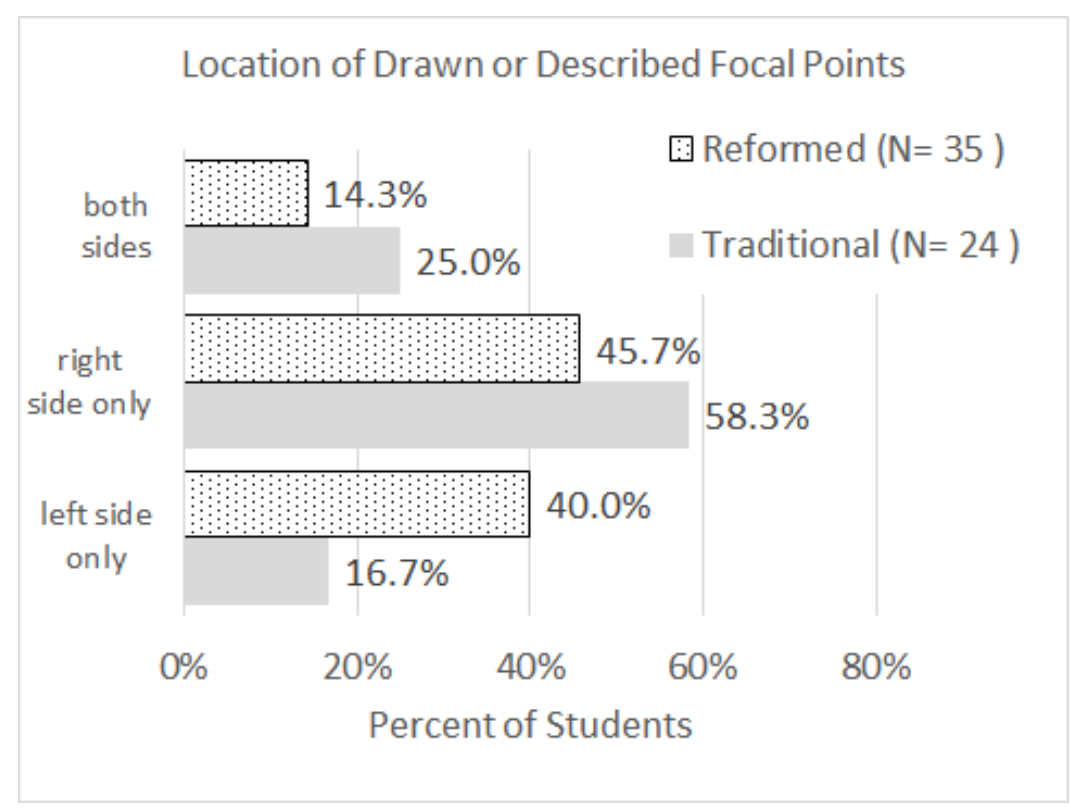

Figure 7.22: Distribution of the locations of focal points as drawn by students.

Five students from the reformed course and six from the traditional course placed focal points on both sides of the lens. Of these 11 students, only three drew what could be a correct diagram of the lights rays as they exit the lens. These three students were all in the traditional course. The other eight students all drew incorrect diagrams similar to the example in Fig. 7.23, where light from the focal point converges on the other focal point after passing through the lens. The correct diagram would show light from the focal point exiting the lens parallel to the optical axis as in Fig. 7.21(a). 


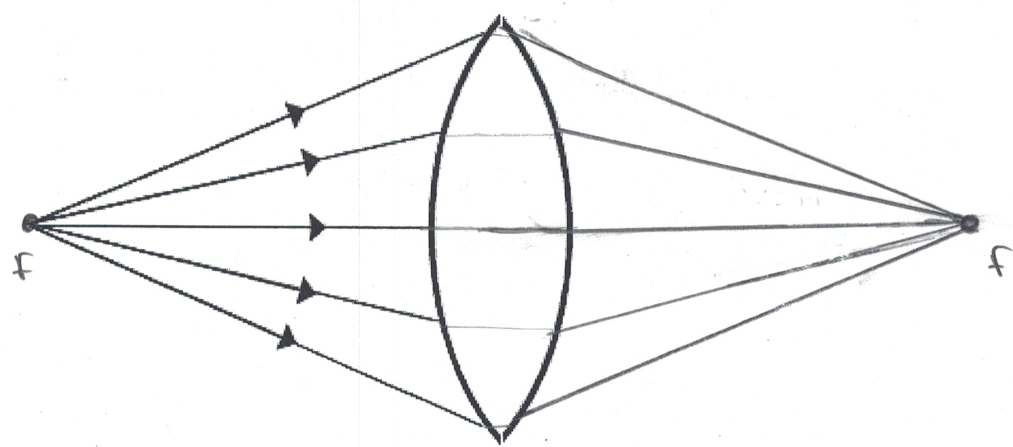

Figure 7.23: Example drawing by a student. The focal points of the lens are drawn on both sides. The student incorrectly depicts light from the focal point on one side converging at the focal point on the other side of the lens.

Fourteen students from the reformed course and four from the traditional course drew focal points only on the left side of the lens. All of these drawings placed the focal point at the light source as in Fig. 7.21(a). Of these 18 students, 16 correctly drew the lights rays parallel to the optical axis. Both of the incorrect drawings came from the reformed course.

Of the students who drew or described the location of a focal point, the most common placement was on the right side of the lens only. 16 students from the reformed course and 14 from the traditional course fell in this category. All but one of these students incorrectly drew the rays converging to the focal point they had drawn. An example of this is shown in Fig. 7.24. This is an incorrect depiction. The rays will converge at the focal point only if the incident rays are parallel to the optical axis. This is evident when considering the inverse case shown in Fig. 7.21(a). The single student who drew a correct image was in the reformed course. 


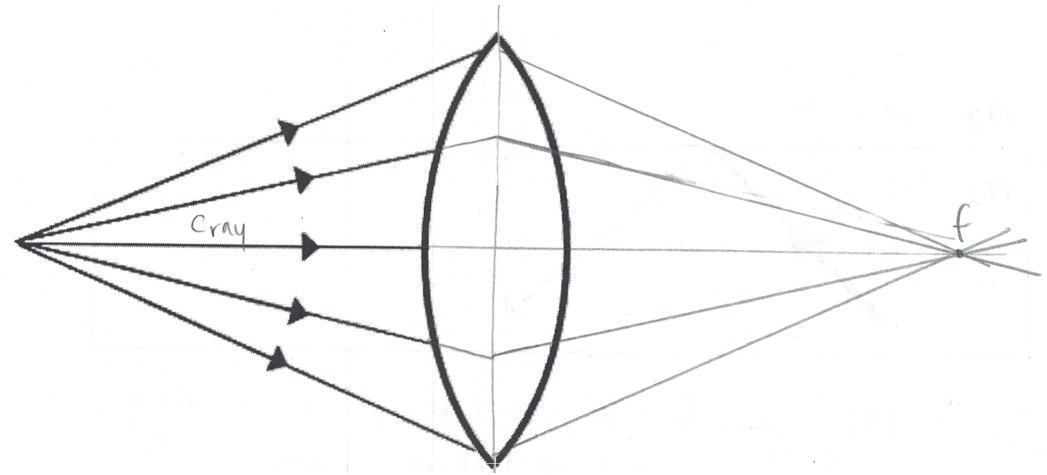

Figure 7.24: Example drawing by a student. The focal point is drawn only on the right side of the lens. The converging rays are incorrectly shown as passing through the focal point.

Using this analysis, the students who drew or described a focal point can be reorganized as having correct or incorrect solutions. The results are shown in Table 7.2 .

Table 7.2: Distribution of students who drew correct solutions to the prompt in Fig. 7.20. The columns are separated by the location of the focal point of the lens.

\begin{tabular}{lccc|ccc} 
& \multicolumn{3}{c}{ Reformed } & \multicolumn{3}{c}{ Traditional } \\
\cline { 2 - 7 } & left & both & right & left & both & right \\
\hline correct & 12 & 0 & 1 & 4 & 3 & 0 \\
incorrect & 2 & 5 & 15 & 0 & 3 & 14 \\
\hline
\end{tabular}

Using the information in Table 7.2 , it may be possible to make inferences about the accuracy of the illustrations that do not specify a focal point. Students who drew focal points only on the left side of the lens were highly likely to have a correct solution. Students who drew focal points on the right side only or on both sides were highly likely to have incorrect solutions. This could mean that we can use the paths of the light rays a proxy for accuracy of the drawings of students who did not include a focal point.

The responses of students who did include a focal length must be compared to those of students who did not include a focal length to suggest the sample of students is 
representative. Figure 7.25 shows the distribution of students' illustrations. Students are separated by whether or not they included a focal length in their answer. They are not separated by class type. Although the students who drew a focal point were more likely to draw converging rays, there was not a significant relationship between groups, $\chi^{2}(3, N=135)=7.63, p=0.054$. It should be noted that the test for independence is only slightly exceeds the criterion for significance of $p=0.05$.

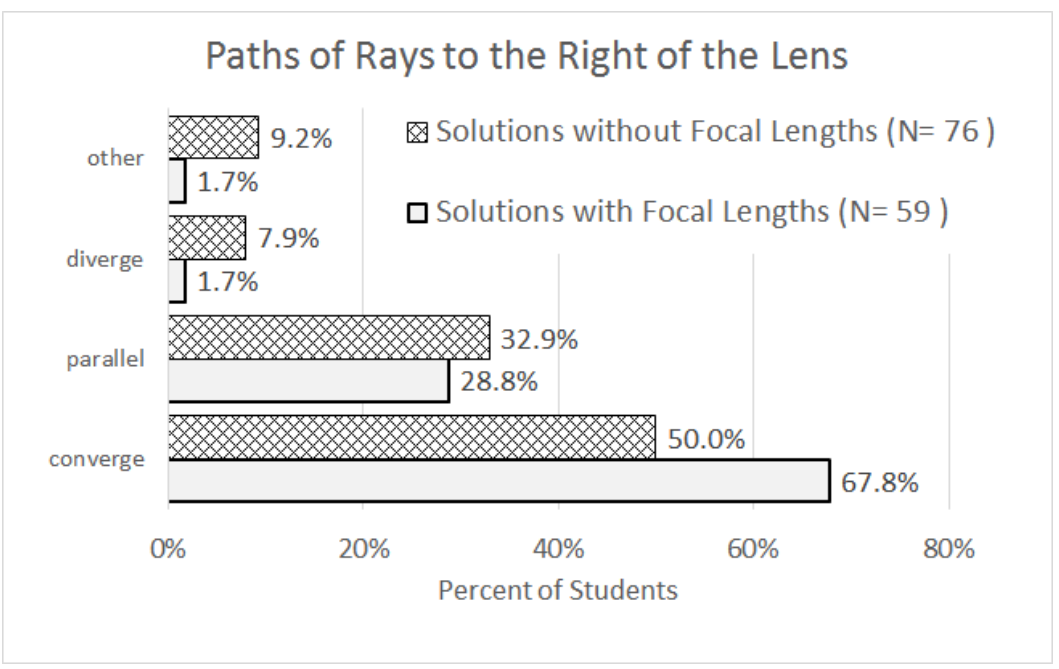

Figure 7.25: Comparison of the paths of light rays as drawn by students who did or did not include focal lengths.

There was a significant relationship between the path of the rays and the course reform, $\chi^{2}(3, N=135)=9.20, p=0.027$. 


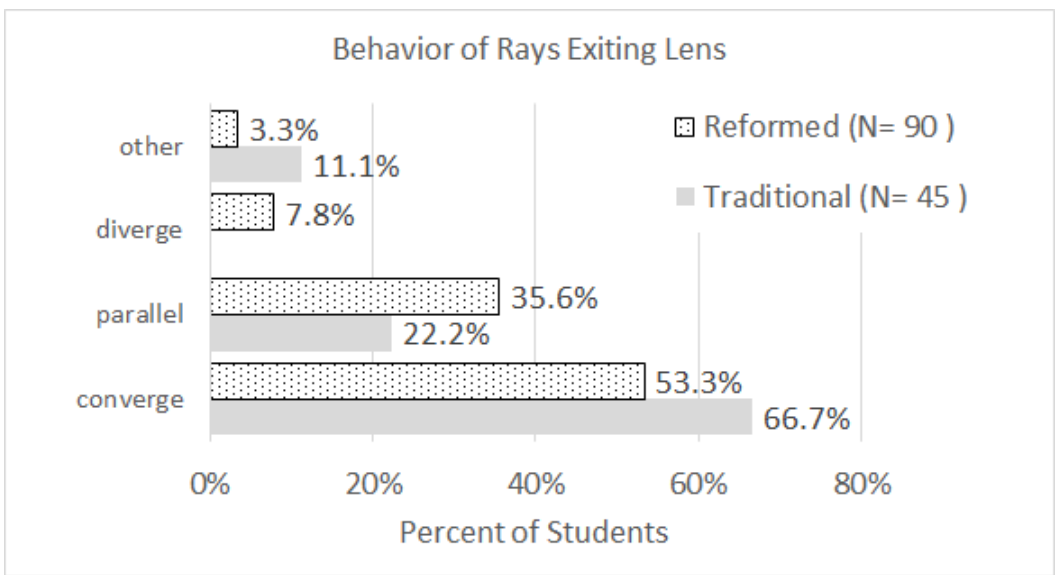

Figure 7.26: Comparison of the paths of light rays as drawn by students in the reformed and traditional courses.

This analysis is not conclusive evidence that the students in the reformed course demonstrated a greater conceptual understanding of image formation. The reasons for this are discussed in the conclusion of this section.

Location of Lens Refraction One of the unexpected trends in this analysis dealt with how students portrayed lens refraction. Figure 7.27 shows three responses from students' quizzes. Physics textbooks and educational material typically portray the location of the refraction of a lens as occurring at either both of the surfaces of the lens or at the principal plane. Parts (a) and (b) show similar depictions respectively. Part (c) depicts the refraction location as only occurring at one side of the lens. This is rarely found in physics texts because it is a misrepresentation of refraction. 


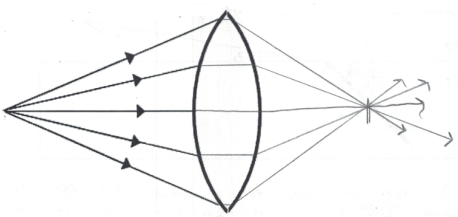

(a)

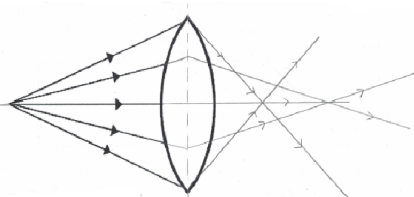

(b)

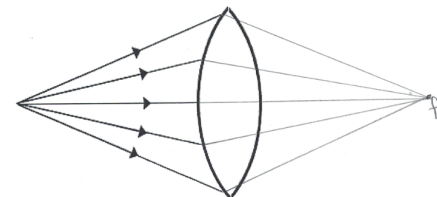

(c)

Figure 7.27: Examples of the location of the lens refraction as drawn by students. The drawings shoe the refraction occurring at (a) both sides of the lens, (b) the principal plane, and (c) the left side of the lens only.

Students in the reformed class were more likely to represent the refraction location as occurring only at the left side of the lens (Fig. 7.28). This is a significant difference when compared to the students in the traditional course who were more likely to use the principal plane $\chi^{2}(4, N=135)=18.76, p<0.001$. The cause of this is unclear. Content created for the reformed course regularly used depictions of refraction occurring at the principal plane.

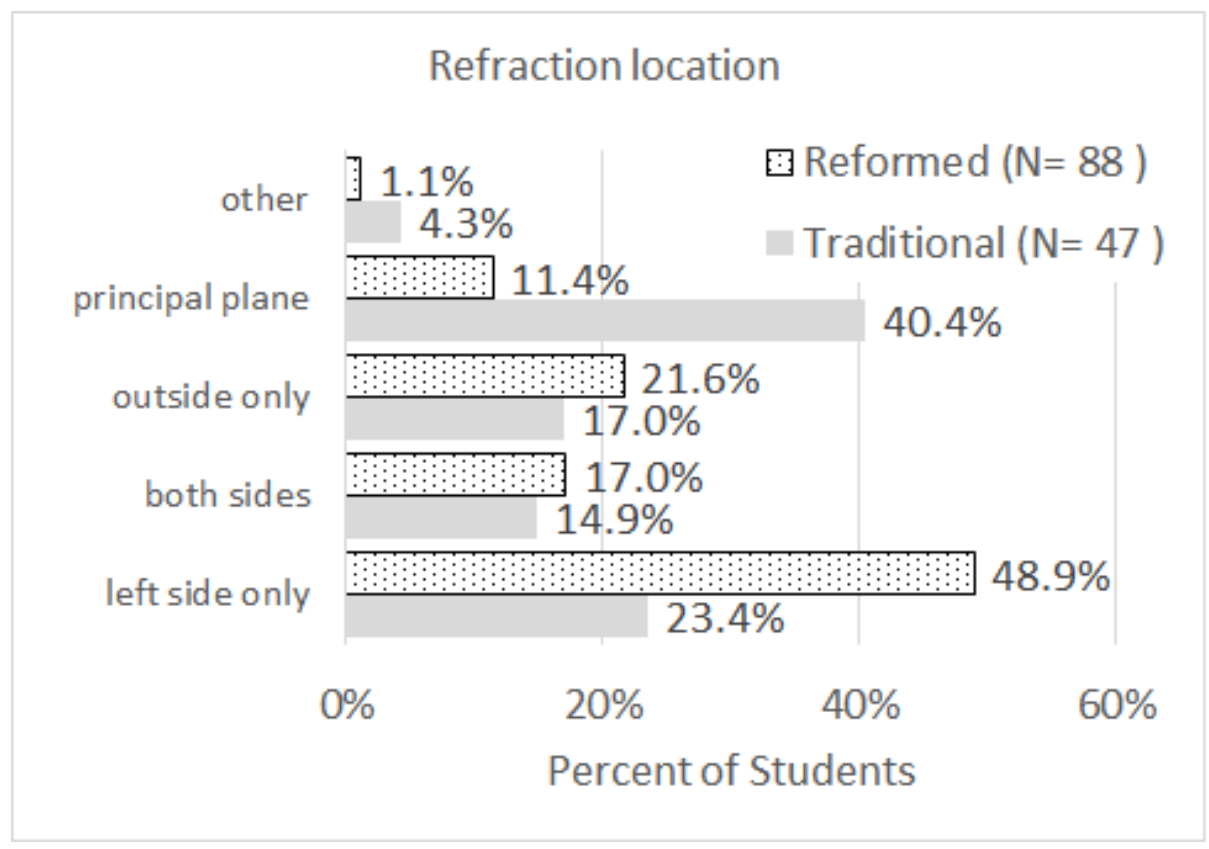

Figure 7.28: Distribution of students' depiction of the location of the lens refraction. Chi-square test of independence resulted in a significant difference in the distributions. $\chi^{2}(6, N=150)=20.3, p<0.01$ 


\subsubsection{Real-World Contextualization}

Figure 7.29 shows the distribution of students' real-world contextualization of the image formation prompt (Fig. 7.20). While students in the reformed course were more likely to cite a medical example $(72.5 \%)$, over half of the students in the traditional course did as well (52.1\%). This is the largest percentage of biomedical applications for either group.

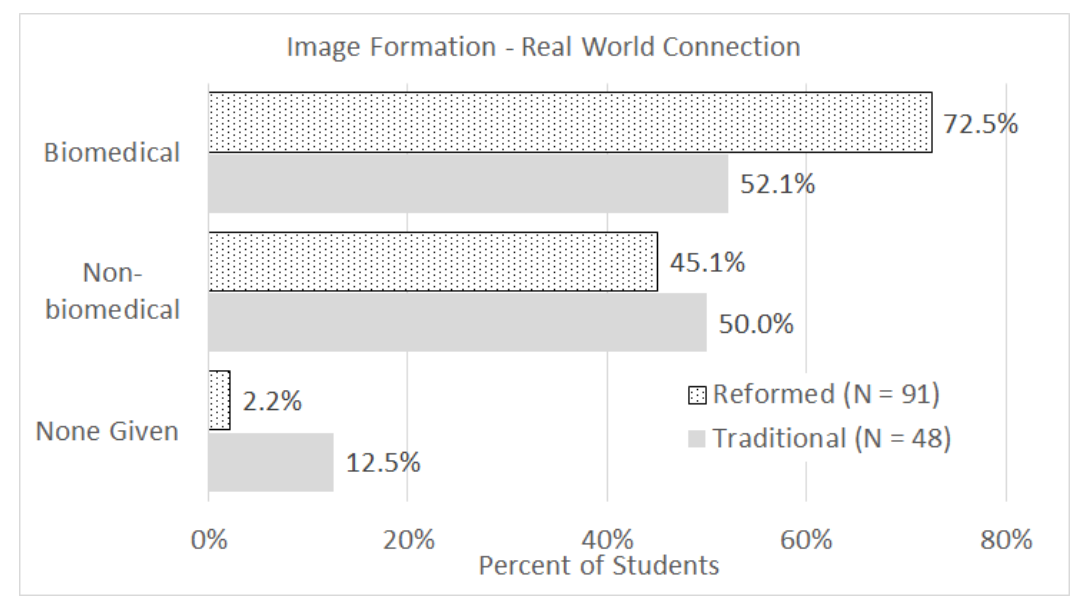

Figure 7.29: Distribution of students' real-world contextualization of the image formation prompt (Fig. 7.20). Many students gave multiple examples, so the percentages do not sum to 100 percent.

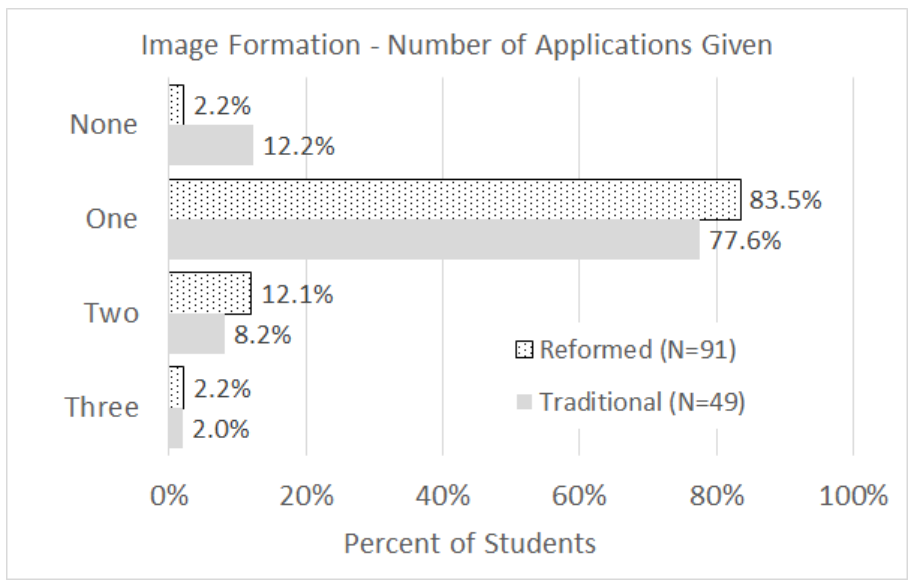

Figure 7.30: Distribution of the number of real-world applications given by students of the image formation prompt (Fig. 7.20). 
The most prevalent biomedical examples in both the traditional and reformed courses were vision defects and corrective lenses. Other biological applications include eye anatomy, LASIK surgery, and the Hartmann-Schack sensor, which measures aberrations in the cornea. Although these examples were almost entirely cited by students in the reformed course. Only one student in the traditional course cited eye anatomy. The non-biomedical category composed primarily of optical devices such as telescopes and magnifying glasses.

The large number of biomedical examples from the reformed course is to be ex-

pected. Students were exposed to multiple examples of the use of lenses in medicine both in lecture and the required laboratory section associated with the course.

Approximately half of the students in the traditional course cited biomedical examples. Students in this course were also exposed to descriptions of image formation by the eye and vision defects. However, much less time was spent discussing these topics than in the reformed course. Two class sessions were spent exploring optical equipment including image formation in the eye and vision defects. Only a portion of that time was focused on biomedical examples. The rest of that time was used to detail other optical technology like magnifying glasses, microscopes, and telescopes. The required laboratory session associated with the traditional course did not include biomedical applications.

\subsubsection{Discussion}

As with the other quizzes, the open-ended nature of the prompt made conclusive analysis between groups difficult. However, unlike the other quizzes the issue should be easily remedied by compelling students to draw the focal point of the lens on their image. The focal point could be included as part of the quiz, but certain 
misconceptions might not be discerned. For example, students may be less likely to draw an image like in Fig. 7.24, where the rays converge at a focal point on the right side of the lens.

Based on the responses of students who included a focal point in their response, an inference was made about students' conceptual understanding. Although there may be evidence that the students in the reformed course demonstrated a greater conceptual understanding, it should be emphasized that this is not a conclusive statement. Rather it is based on inferences using the analysis of students who included a focal point. Students who did not include a focal point might not have made the same errors as those who did include the focal point. The reason the students who drew focal points and converging rays were often wrong is because they had the rays converging at the focal point. It is quite possible that the students who did not label the focal point is because they knew that the rays would converge at a different location.

Students in the reformed course were significantly more likely to draw the refraction as only occurring at the left side of the lens. It is possible that these students were thinking of the depiction of the lens of the eye. Before contacting the retina, light that enters the eye goes through multiple mediums: the cornea, the aqueous humor, the lens, and the vitreous humor. There is a refraction at each of the boundaries between these mediums because they all have different refractive indexes. However, the differences in refractive index between the mediums are very small. This means that the refractions are very small. The largest refraction happens at the air-cornea interface. Therefore depictions of light entering the eye do not always depict refractions at locations other than at the cornea. A small number of students even explicitly drew eyeballs around the lens given in the prompt.

Students in both courses showed a high probability of citing a biomedical real- 
world example in their response. The most prevalent application for both groups was vision defects and corrective lenses. While both groups received instruction on this topic, the reformed course objectively spent more time exploring it in both the lecture and lab sessions. This may explain why the students in the reformed course were still more likely to cite a biomedical application than students in the traditional course.

\subsection{Discussion}

The open-ended nature of the prompts was as a necessity if students' contextualization of the physics concept in the illustration was not going to be influenced. However, this same feature resulted in a wide variety of responses, many of which lacked enough detail for conclusive analysis. It is possible that we have been able to glean whether students are framing these phenomena in the context of biomedicine. But it is less clear whether that context has had an impact on their conceptual understanding.

The results from the real-world application analysis consistently show that a greater percentage of students from the reformed course contextualized the physical phenomena in biomedicine. $30.5 \%$ and $58.7 \%$ percent of the students from the reformed course cited biomedical applications for the sound waves and TIR quizzes respectively. Whereas almost none of the students from the traditional course gave a biomedical example for these quizzes at all. Over twenty percent of the students in the traditional course did not even cite a single non-biomedical example for the sound waves quiz. Although $16.7 \%$ of students in the traditional course cited a biomedical example for the thin film interference quiz, nearly twice the percentage of students from the reformed course (29.8\%) also cited biomedical applications. Both the traditional and reformed courses discussed vision defects. However, the reformed course spent a great deal more time on the topic than the traditional course. This may 
explain why half of the students in the traditional course cited a biomedical example in the image formation quiz compared to three quarters of the reformed course.

While it is difficult to state conclusively whether one cohort demonstrated greater conceptual understanding than the other, there are only minimal signs that a difference in understanding exists between students in these courses. Students in the reformed course were more likely to mention the Doppler shift than the students in the traditional course. However those who did describe it, did so correctly regardless of instructional method. Students in the reformed course were more likely to incorrectly draw refractions only at the top surface of a lens in the image formation quiz. However, there is inferential evidence that suggests they were more likely to correctly describe image formation by a lens. They were also more likely to incorrectly draw the angles of refraction and reflection with respect to the surface in the TIR quiz. Students in the traditional course were more likely to not draw a refraction of light passing into a different medium on the thin film quiz. However most of these same students correctly drew refractions in the TIR quiz.

Future versions of these illustration-based quizzes will benefit from the analysis described in this chapter. Each quiz should be modified so that the illustrations are slightly more specific. Students should be explicitly told to provide quantities like index of refraction or focal points. They should also be incentivized to defend their responses with the correct equation that describes the phenomenon. The openended nature should be maintained, but slight modifications should greatly improve researchers' ability to probe students' conceptual understanding.

Although correlations between student understanding and contextualization could not be performed due to the wide variety of responses, this should remain a goal for future iterations. It will also be valuable to run similar tests on a physical concept that 
is not framed in a biomedical context in either course. In the past, the introductory courses have discussed aspect of relativity and quantum mechanics. Although both topics have medical applications discussed in the intermediate course, content has not been developed that is appropriate for the introductory course. Students' responses to these topics could demonstrate whether any differences in conceptual understanding are actually due to biomedical contextualization.

There is clear evidence in this chapter that students' contextualization of different physical phenomena was impacted by the course reforms. What is less clear is what effect, if any, this had on students conceptual understanding. 


\section{Discussion}

Attitude and motivation are important components of the affective domain [47-56]. In order for students to develop good attitudes and strong motivation for content, they must transition from a short-term situational interest to a deeper individual interest in the material $[47,50,55,56]$. Individual interest requires that the students recognize utility value in what they study [48]. Therefore, favorable attitudes toward physics can develop when students see practical value in what they learn in class. The course reforms developed in this project were designed to provide that connection for prehealth students. This chapter will discuss how the results of the study demonstrate the impact of biomedically-focused course materials on students' attitudes and the implications for future research at PSU and by the IPLS community.

The hypothesis explored in this research is:

By participating in the reformed course, pre-health students will exhibit increased levels of interest in physics and be able to accurately describe physical concepts in a biomedical context.

The individual research questions addressed in the hypothesis are,

1. Are student opinions on the relevance of physics impacted by biomedicallyfocused physics instruction?

2. Do students learn core physics principles and make links to biomedical applica- 
tions from the curriculum?

The evidence collected for these questions is addressed in the following sections.

\subsection{Research Question 1: Opinions on the Relevance of Physics}

There is a clear evidence that students' attitudes toward physics instruction was impacted by the biomedically-focused instruction. However, there were other factors beyond the course reforms that may have influenced the results.

The majority of students stated that the course reforms were an improvement in physics instruction. They felt this way even though they felt the course required a larger time commitment than previous courses. Students' reported that the biomedical context made it more enjoyable to study physics and easier to understand. They indicated that it is easier to engage with physics topics when they are presented by biomedical professionals.

Many students indicated in the surveys and in the interviews that they had not seen the connection between physics and medicine until the reformed course in spring. Some students stated that it had not occurred to them to associate the two while others explicitly stated that there was no relationship. Having made this connection in spring term, students said that they had started to see connections elsewhere. Some students said that they were able to see applications to content covered in fall term, even though it had not been presented in a biomedical context. Other students commented that they had felt a stronger connection with the spring material because it dealt with technology with which they have professional experience. Students in the interview mentioned talking about the physics behind the technology with their co-workers. 
Attitudinal shifts as measured by the CLASS showed that students in the traditional course had more shifts toward expert-like responses than students in the reformed course. It is true that students in the reformed course had a greater percentage of expert-like responses both at the beginning and end of spring term in almost all categories. However, the effect size of the shifts from both cohorts suggest that the traditional section did in fact have more expert-like gains.

The course reforms are meant to improve students' attitudes toward the physics by highlighting how it is involved in their future careers. Therefore it is important to consider whether the questions of the CLASS are adequately addressing this. The results of the rewritten CLASS questions suggest that students are not interpreting phrases like 'everyday life' and 'the real world' as pertaining to their careers. Similar results were seen in the interviews. When asked whether they think about the physics they experience in everyday life, students failed to supply any medical examples.

Many aspects of the study suggest that students' attitudes toward physics improved over the course of the year. After winter and spring term, students were more and more likely to think that physics should be a required course. They were also more likely to state that they enjoyed talking about the course content outside of class. Students in both groups were able to give more examples of the relationship between physics and medicine by the end of the year than at the beginning. It is possible that course reforms were building on these improving attitudes. Adopting biomedically related content in fall term may results in even larger shifts than those seen in spring. 


\subsection{Research Question 2: Conceptual Understanding and Contextualiza- tion}

There is clear evidence that students' contextualization of certain physical phenomena was impacted by the biomedical reforms. And while it is unclear to what extant this also impacted their conceptual understanding of those same phenomena, few significant differences were found between the two cohorts.

In each if the four quizzes, students in the reformed course were more likely to cite a biomedical application than students in the traditional course. The greatest differences were observed in the TIR and sound waves quiz. $30 \%$ of the reformed course cited a biomedical example for the sound waves and $59 \%$ for the TIR quiz. Only a few students in the traditional course mentioned any biomedical applications on these quizzes at all. Roughly $30 \%$ of the reformed course and $17 \%$ of the traditional course described a biomedical example in the thin film interference quiz. This is phenomenon used in certain kinds of microscopy. While microscopes were discussed in both courses, the reformed section spent more time on it than the traditional course. The largest percentage of biomedical examples from either group was seen in the image formation quiz. $73 \%$ of the reformed course and $52 \%$ of the traditional course mentioned different forms kinds of vision defects or corrective lenses. The fact that this was a popular category for both groups is not surprising, since they were discussed in both courses. However, students in the reformed course had a great deal more instruction in these topics. This may explain why they were more likely to cite vision defects in their answers.

As stated above, measuring conceptual understanding through these quizzes was less straightforward than the analysis of the real-world connections. The open-ended 
nature of the quizzes was chosen to avoid influencing students' contextualization. However, this allowed for a wide variety of responses that were at times difficult to analyze.

Even though conclusive statements about the conceptual understanding of either group can not be made, the analysis of students' responses differed in various aspects. Notably, the students in the reformed course were more likely to take angles with respect to the surface than from the normal in the TIR quiz. They were also more likely to draw refractions at the surface of the lens rather than at the principal plane.

\subsection{Implications for IPLS Research}

Many aspects of this study were designed to further elucidate research carried out by other IPLS developers. Many trends observed in those studies reflect the findings presented here, notably that the majority of students responded favorably to IPLS course reforms. $[28,33,34,58]$ There are also features that have yet to be extensively studied, but should be explored by further research.

\subsubsection{Pre-health Students' Attitudes toward Physics}

IPLS researchers have identified improving students' interest in physics and demonstrating its relevance as unsolved challenges [43]. Studies of reformed IPLS courses found that students responded favorably. Students stated that the reforms helped them see the usefulness of physics in understanding the life-sciences $[28,33]$. The reforms also made physics more enjoyable [58] and helped students see the relevance

of physics to biology and medicine $[34,58]$. One study found that life science students gave the IPLS reforms high ratings even though they also indicated that it required 
more time on course work [34].

The results of this study find similar positive impacts of the biomedically-focused course reforms. Students stated that the course was an improvement in physics instruction even though it required more work outside of class. The majority also stated that the biomedical applications helped them enjoy and understand physics more than traditional instruction. Students indicated that the reforms helped them see connections between physics and medicine more so than in the traditional fall and winter terms.

Even though the student response to IPLS reforms are largely favorable, some students had a mixed reaction. They stated that they would have preferred more traditional instruction $[34,58]$. This appears to have been due to a feeling of missing out on other topics because of the focus on biological applications. The students surveyed and interviewed in the current study cited similar reasons for potentially favoring traditional instruction. Although this feeling does not necessarily mean that these students were not in favor of the reforms. Similar comments were seen from students who gave the course high ratings. These students suggested that they would have preferred a mix of biomedical and traditional applications.

The results from this study and others show that life science and pre-health students are generally in favor of more explicit biological and biomedical applications of physics. This is certainly an encouraging trend and increased student interest should be considered a significant improvement in instruction. However there is insufficient evidence to state whether these applications are only promoting a short-term situational interest or the deeper individual interest that can develop from a perceived utility value. 


\subsubsection{Utility Value of Physics to Life Science Students}

One of the goals of the IPLS community is demonstrating the utility value of physics to life science students, even though this is not usually explicitly stated in the literature. Research in the development and assessment of IPLS courses has tended to focus on terms such as relevance or authenticity [58,62]. Recommendations from expert committees have also cited demonstrating the relevance of physics as one of the unsolved challenges in the IPLS field [43]. Medical organizations have stated that being able to apply physical principles to living systems should be considered an important competency for students entering medical education [2]. Citing the application of relevant and authentic physical principles as a competency for pre-health students is tacitly stating the importance of demonstrating the utility value of physics.

Studies of IPLS courses indirectly probed the idea of utility value with survey questions that asked about the relevance or usefulness of physics to biology and/or medicine $[28,34,58]$. These questions and others like them begin to probe students' views of the utility of physics concepts and/or methods, but do not describe what is likely a nuanced attitude. In one study, for example, students were asked two questions on a five point Likert scale. The questions asked students whether they found the biological applications to be (1) interesting and (2) relevant to their other courses and/or future career [58]. While nearly $80 \%$ agreed that they found the applications interesting, less than $60 \%$ agreed that the applications were relevant. This potentially addresses the difference between the development of situational and individual interest, but questions still remain. When students state something is relevant to their future career, what exactly do they mean? Do they believe that they will be actively using the physics content they learn in class? 
Something similar was seen in this study. For example, many students stated in surveys and interviews that the biomedical content was more relevant or applicable to their future career. However, that did not necessarily mean that they felt that they would really need to regularly apply that knowledge. One reason cited was that practitioners would not need a detailed understanding of medical technology in order to use it. Students pursuing a career in physical therapy also indicated that the reforms were more interesting, even though they stated the technology covered in the spring reformed course was not likely to be used in their profession. Examples like this suggest that for some students, the course reforms could have been more successful in promoting short-term situational interest rather than deeper personal interest fostered though perceived utility value.

Engendering situational interest in physics should certainly be regarded as a positive result in any intervention. However, achieving the implicit goal of demonstrating utility value requires more explicit research into the impacts of course reform.

\subsubsection{Use of the CLASS by IPLS Researchers}

The CLASS is a tool commonly used by researchers for measuring attitudinal shifts as a result of instruction [96]. It was developed for use in all physics courses including non-IPLS classes [60]. Because of this, it might not be adequately measuring shifts in the attitudes IPLS course are attempting to modify.

This sentiment has been expressed by other IPLS researchers exploring the impact of course reforms on students' attitudes $[33,58]$. In one study, students' score on the real-world connection category of the CLASS shifted by $-1.3 \% \pm 2.8 \%$ [58]. The investigators acknowledged that this was not a significant shift, but pointed out that this conflicted with responses on student surveys. In that survey, $28 \%$ of students 
agreed with the statement "It has become much more evident to me how much biology is dependent on physical principles." The researchers stated that one of the possible reasons for this disparity was due to the broad nature of the CLASS.

The CLASS is not focused on biology in particular, so students may have been thinking about other applications (e.g., cars, toaster ovens) about which their opinions did not change. [58]

The results in this study of the rewritten CLASS questions seem to support this idea (Sec. 4.4). The rewritten questions replaced phrases such as 'everyday life' and 'the real world' with phrases like 'my future career'. The results of these rewritten questions often differed from the original CLASS questions. However, only limited conclusions can be drawn from these results given that the questions used in this study did not undergo the same rigorous validation process that the developers of the CLASS carried out.

Stronger claims could be supported with additional data from future iterations of the reformed course. Furthermore, IPLS researchers would benefit from further explorations or possible alterations to these attitudinal questions.

\subsubsection{Accounting for Gains in Favorable Attitudes}

Multiple attitudinal aspects in this project, including the CLASS, suggested that students' attitudes were improving over winter and spring term. Improvements in CLASS scores during the year has been observed by other researchers. The original study used in the development of the CLASS looked at the shifts in students' attitudes over two semesters: fall and spring. They found that shifts in students' attitudes were

less favorable in the fall semester than they were in the spring semester [9]. In a study of IPLS reforms, researchers found that a reformed course in the spring semester had 
more shifts toward expert-like behavior than the traditional course offered in the fall semester [57]. If students' attitudes are already improving during the year, it may be difficult to separate those gains from gains that result from course reforms.

The shift away from expert-like beliefs at the beginning of the year also suggests that course reforms in fall term could have the largest impact. In the same IPLS study mentioned above, researchers found that the largest attitudinal gains at the end of the semester were made by students that had the lowest CLASS scores at the beginning of the semester [57]. In another study, the MPEX was used to assess shifts in attitudes due to an IPLS course offered in the fall semester [33]. They did not find significant shifts as a result of the course, however this was actually an improvement over the results of previous traditional courses offered in the fall semester, all of which resulted in large, significant shifts away from expert-like responses.

Like the studies mentioned above, we found that students' attitudes in fall term showed large losses in expert-like beliefs, but improved during the year. Why are the results of fall term worse than for winter and spring? One possibility could be the decreasing number of students each term. A meta-analysis of CLASS data found that a smaller class was more likely to results in favorable shifts. [96] The idea that students with unfavorable attitudes were more likely to withdraw from physics courses as the year progressed does not appear to have been the cause since matched student data throughout the year shows a similar trend of improved attitudes. Another factor could be that students had to acclimate to the many demands placed on them in fall term, including the focus on applied mathematics. Future research should explore the effect of reformed fall and winter term courses at PSU. In the 2017-2018 school year, selected biomedical topics will be added to both of these courses. Multimedia curriculum for physical therapy and the cardiovascular system have been developed 
at PSU for fall and winter term, but more materials are necessary to reform the entire terms. Surveys of students in these courses may reveal additional information.

\subsubsection{Physics Content for Pre-Health Students}

The life-science students that are meant to benefit from IPLS reforms have a diverse range of backgrounds and career aspirations. Because of this, researchers have stated that there is not a single IPLS course that could equally serve all life-science students $[28,43]$. Therefore an important goal of the IPLS community is to develop a wide variety of course materials that can be made available to educators who can decide what content is most appropriate for their students [43].

The content developed in this project adds to the existing resources for instructors of IPLS courses. The materials are designed to be of particular relevance to prehealth students. The modular nature of the topics covered allow for easy adoption in whole or in part. The multimedia materials offer instructors multiple methods of incorporating biomedically relevant content in introductory and intermediate level physics courses.

\subsubsection{Adoption by Instructors of Traditional Physics}

Adopting course reforms can be a large challenge for any instructor. One of the concerns raised by IPLS researchers is whether content with a focus on biology or medicine can be readily adopted by instructors with little experience in these fields $[28,33,43]$. The reforms developed by this project were designed with that challenge in mind.

We believed that content exploring biomedical technology would be something an 
instructor with a traditional physics background could more readily adopt. This is because instrumentation is a common factor in both biomedicine and physics. Based on the comments by the instructor of the reformed 2016 spring term course, this was largely true. The instructor did state though that he occasionally received medicallyfocused questions that were difficult to answer. However, this was not a common occurrence. Students tended to focus on the physical concepts being discussed. Future instructors who adopt these reforms will also benefit from the large amount of lecture material developed for this project, including material created by the instructor of the 2016 spring term course.

\subsubsection{Influence of Peers and Professionals on Student Attitudes}

Multiple students indicated in the surveys and the interviews that their opinions of physics instruction were affected by comments from others. Some had spoken with students who had taken physics in a previous year. They were told that the course would be difficult or not relevant to their overall education. Other students mentioned speaking with professionals in their field. Those professionals told the students that physics had not played a role in their careers. It would seem likely that students who have yet to take a physics course, and therefore do not yet have firm opinions on physics instruction, might rely on the views of their peers. This could cause students to hold negative views of physics before class even starts.

Education research has found that the teacher can be an important factor in developing motivation on students [47]. However, student motivation can also be influenced by agents outside of the classroom. One study of high school students explored this idea by introducing a motivational intervention to a random selection of parents [115]. The intervention consisted of a brochure and website sent to parents which detailed 
"the utility of mathematics and science courses for their children's futures" [115].

The researchers found that parents that received the intervention had a significantly higher perceived utility value of mathematics and science courses. They also found that children of those same parents also had a significantly higher perceived utility value and enrolled in more mathematics and science courses in their final two years of high school.

The question IPLS research should address is whether students' attitudes toward the relevance of physics are being influenced by agents outside of the classroom and who the outside agents are. Comments from students in this study suggest the outside agents that could have had the largest impact on attitudes toward the relevance of physics in medicine are other pre-health students and professional colleagues. IPLS researchers have suggested that academic advisers may also have influence over student attitudes [1].

If these agents are found to have a significant impact, the IPLS community would benefit from determining interventions to positively impact this influence. The results of this study suggest that the biomedical expert interviews helped students see the relevance of physics to biomedicine. Similar videos could therefore be a tool to reverse low levels of perceived utility value.

\subsubsection{Viewership of Biomedical Expert Interviews}

In the chapter that dealt with the response to the biomedical focus (Sec. 5.2) many students indicated that the interviews with the biomedical experts were an important part of their increased appreciation for the relationship between physics and biomedicine. However, some students commented that they did not watch videos toward the end of the term. This trend was observed in viewership data collected 
though FlipItPhysics. A similar decrease in viewership during the course of the term was observed by the creators of the original FlipItPhysics content [114].

However, the data in this report suffers from being an incomplete record of students' interactions with the videos. Students that view the video in one window and answer questions in a separate window at the same time might not have been categorized as viewers. Understanding the extent of the impact of the biomedical interviews requires a better measure of students' interactions with the videos. Is the change in behavior due to students becoming more efficient by using multiple windows? Or are there students who become less engaged in the content because of the perceive lack of value the course places on the videos? Future iterations of the reformed course could explore additional ways of tracking student behavior through software developed by Google Analytics [116] or through more detailed surveys and interviews. 


\section{Conclusion}

Pre-health students are often required to take introductory physics courses as undergraduates. These students often struggle with this requirement as it is not always apparent how the concepts covered in an introductory physics course will benefit them in their future careers. PSU has developed curriculum for introductory and intermediate level physics courses that strive to make those connections by teaching physics in a biomedically relevant context.

The laboratory activities that were developed as part of this project focus on exploring the physical principles behind common medical devices or concepts. The BIA activity leads students through how body composition can be estimated through measurement of electrical currents. This required researching, designing, and building a device to make these measurements in the classroom. The CT project demonstrates to students how an opaque object can be imaged using invisible light. For this activity, both the equipment and software necessary for the lab were created. The additional laboratory activities developed for this project explored vision, radiography, pulse oximetry, electrocardiograms, and body mechanics. All of these labs were modified for adoption in either the introductory or intermediate courses. Their use in the classroom has been assessed including through pre/post quizzes.

The multimedia modules show the relationship between physics and medicine through video interviews with biomedical experts, original texts, and relevant home- 
work questions. In each of the video interviews, biomedical experts detail how physics is used in their fields. The texts written for the modules serve as a fundamental scientific introduction to the physical concepts and technical discussions of their application in biomedicine. An online homework platform integrates the material, stimulating deeper exploration and engagement from a conceptual standpoint with quantitative calculations and qualitative problem solving. Detailed analysis of the multimedia modules was conducted using original python code and students' log data from the FlipItPhysics platform. This analysis has been used to improve the content and clarity of the modules.

The study of the reformed curriculum explored the impact these reforms had on the attitudes of pre-health students toward physics instruction. Attitudes were assessed with the CLASS, course surveys, student interviews, and conceptual quizzes. The results indicate that students' attitudes were impacted in multiple ways, though there is evidence that other factors also played a role.

Data from the CLASS shows that in spring term students in the traditional course had larger favorable shifts in expert-like responses than students in the reformed course. However, students in the traditional course had a higher percentage of expertlike responses going into the term and remained higher at the end of the term. Furthermore, data from the rewritten CLASS questions suggest that the original questions might not be measuring shifts in attitudes about how students' value physics in their future careers. Analysis of the full year of CLASS results suggest that other factors like course meeting times and students' development over the year may have impacted the results.

The survey and interview results show that a majority of the students approved of the course reforms even though it required a larger time commitment. Many of these 
students expressed that they struggled to see the connection between physics and their future careers in the health sciences until spring term. They stated that including biomedical content and information from biomedical experts made the course more enjoyable. A portion of the students remained skeptical of the reforms. Even though they stated that the biomedical focus improved the course, some of them felt that the detailed physics knowledge they learned in the course would not actually be needed on a practical basis as a medical professional. Other students felt that they were missing the broader scope of topics and ideas they encountered in the traditional course. Evidence was also shown that students' attitudes seemed to be improving over the course of the year, suggesting that the largest impact of course reforms could be seen in fall term. This could be explored when the additional biomedical material is added to fall and winter terms in the 2017-2018 school year. At PSU a module on physical therapy will be added to the algebra-based introductory course in fall term, and a module on the cardiovascular system will be added to the winter term course.

The results of the illustration-based quizzes clearly showed that students' realworld contextualization of certain physical phenomena was impacted by the course reforms. In all four quizzes, students in the reformed course were more likely to cite biomedical applications than students in the traditional course. Virtually no students from the traditional course mentioned biomedical examples for the sound waves or total internal reflection quizzes, probably because they did not receive instruction that presented those ideas in a biomedical context. Students in the traditional course did learn certain biomedical applications of the thin film interference and image formation topics, and this was reflected in the real-world applications they cited. However, the students in the reformed course were still more likely to describe a biomedical application. This is not surprising since they spent a great deal more time exploring biomedical connections. Measurements of conceptual understanding through the 
quizzes was more difficult to perceive. The open-ended nature of the quizzes resulted in a wide variety of responses that were difficult to fully analyze. However, few significant differences between the cohorts were found. Students in the reformed course were more likely to incorrectly place take the angle of refraction with respect to the surface boundary than the normal. They were more likely to draw lens refraction only at the surface of the lens rather than at the principle plane. Students in the traditional course were more likely to fail to draw a bend due to refraction in the thin film interference quiz.

This study explored the development and impact of reformed curriculum on the attitudes of pre-health students toward physics. The curriculum included laboratory activities and video interviews with biomedical experts as well as original text and homework that explore physics in biomedicine. Direct responses from the students indicated that they approved of the course reforms and appreciated the inclusion of biomedically relevant content. They stated that the course had helped them to make connections with physics that they were not able to make in previous physics classes. 


\section{Bibliography}

[1] Dawn C Meredith and Edward F Redish. Reinventing physics for life sciences majors. Physics Today, 66(7):38, 2013.

[2] AAMC-HHMI Committee et al. Scientific foundations for future physicians. Washington, DC: Association of American Medical Colleges, 2009.

[3] Report IV contemporary issues in medicine: basic science and clinical research. American Association of Medical Colleges, Washington, DC, 2001.

[4] Thomas J Lawley, Jonathan F Saxton, and Michael ME Johns. Medical education: time for reform. Transactions of the American Clinical and Climatological Association, 116:311, 2005.

[5] Catherine H Crouch, Robert Hilborn, Suzanne Amador Kane, and Timothy McKay. Physics for future physicians and life scientists: a moment of opportunity. APS News, 19(3), March 2010.

[6] Julia Svoboda Gouvea, Vashti Sawtelle, Benjamin D Geller, and Chandra Turpen. A framework for analyzing interdisciplinary tasks: implications for student learning and curricular design. CBE-Life Sciences Education, 12(2):187-205, 2013.

[7] Richard W Moore and Rachel Leigh Hill Foy. The scientific attitude inventory: A revision(SAI II). Journal of Research in Science Teaching, 34(4):327-336, 1997.

[8] Edward F Redish and David Hammer. Reinventing college physics for biologists: Explicating an epistemological curriculum. American Journal of Physics, 77(7):629-642, 2009.

[9] Wendy K Adams, Katherine K Perkins, Noah S Podolefsky, Michael Dubson, Noah D Finkelstein, and Carl E Wieman. New instrument for measuring student beliefs about physics and learning physics: The colorado learning attitudes about science survey. Physical Review Special Topics-Physics Education Research, 2(1):010101, 2006. 
[10] Robert C Hilborn and Michael J Friedlander. Biology and physics competencies for pre-health and other life sciences students. CBE-Life Sciences Education, 12(2):170-174, 2013.

[11] National Research Council (US) Committee on Undergraduate Biology Education to Prepare Research Scientists for the 21st Century et al. BIO2010: Transforming undergraduate education for future research biologists. National Academies Press (US), 2003.

[12] Carol A Brewer and Diane Smith. Vision and change in undergraduate biology education: a call to action. American Association for the Advancement of Science, Washington, DC, 2011.

[13] Susan R Singer, Natalie R Nielsen, Heidi A Schweingruber, et al. Discipline-based education research: understanding and improving learning in undergraduate science and engineering. National Academies Press, 2012.

[14] Terry Woodin, Helen Vasaly, Duncan McBride, and Gary White. Integration of physics and biology: Synergistic undergraduate education for the 21st century. CBE-Life Sciences Education, 12(2):120-123, 2013.

[15] Alastair Baker. Crossing the quality chasm: a new health system for the 21st century. BMJ, 323(7322):1192, 2001.

[16] Matthew L. Ward. Cancer risks debated for type of x-ray scan. The New York Times, Jan. 2010.

[17] Rebelo Bogdanich W. Bad x-rays found again at a brooklyn hospital. The New York Times, April 2011.

[18] Darrell G Kirch, Karen Mitchell, and Cori Ast. The new 2015 MCAT: testing competencies. JAMA, 310(21):2243-2244, 2013.

[19] Robert C Hilborn. Physics and the revised medical college admission test. American Journal of Physics, 82(5):428-433, 2014.

[20] Alexander Stone Macnow, editor. MCAT 2015: What the Test Change Means for You Now. Kaplan Publishing, 2014.

[21] David Sidebottom. Use of bratwurst sausage as a model cadaver in introductory physics for the life sciences lab experiments. The Physics Teacher, 53(6):367-371, 2015.

[22] Dean Zollman, Dyan McBride, Sytil Murphy, Bijaya Aryal, Spartak Kalita, Johannes VD Wirjawan, Boonchoat Paosawatyanyong, and Pornrat Wattanakasiwich. Teaching about the physics of medical imaging. In AIP Conference Proceedings, volume 1263, page 3, 2010. 
[23] Kimberly Moore, J Giannini, and Wolfgang Losert. Toward better physics labs for future biologists. American Journal of Physics, 82(5):387-393, 2014.

[24] Timothy A Stiles. Ultrasound imaging as an undergraduate physics laboratory exercise. American Journal of Physics, 82(5):490-501, 2014.

[25] Yvonne Klapper, Karin Nienhaus, Carlheinz Röcker, and G Ulrich Nienhaus. Lipid membranes and single ion channel recording for the advanced physics laboratory. American Journal of Physics, 82(5):502-509, 2014.

[26] Ivan G Darvey. A simple inexpensive procedure for illustrating some principles of tomography. The Physics Teacher, 51(5):298-299, 2013.

[27] Michael Plomer, Karsten Jessen, Georgi Rangelov, and Michael Meyer. Teaching physics in a physiologically meaningful manner. Physical Review Special Topics-Physics Education Research, 6(2):020116, 2010.

[28] Catherine H Crouch and Kenneth Heller. Introductory physics in biological context: An approach to improve introductory physics for life science students. American Journal of Physics, 82(5):378-386, 2014.

[29] EF Redish, C Bauer, KL Carleton, TJ Cooke, M Cooper, Catherine Hirshfeld Crouch, BW Dreyfus, BD Geller, J Giannini, J Svoboda Gouvea, et al. Nexus/physics: An interdisciplinary repurposing of physics for biologists. American Journal of Physics, 82(5):368-377, 2014.

[30] Anne-Marie Hoskinson, Brian A Couch, Benjamin M Zwickl, Kathleen A Hinko, and Marcos D Caballero. Bridging physics and biology teaching through modeling. American Journal of Physics, 82(5):434-441, 2014.

[31] Nelson Christensen. Medical physics: the perfect intermediate level physics class. European Journal of Physics, 22(4):421, 2001.

[32] Benjamin W Dreyfus, Julia Gouvea, Benjamin D Geller, Vashti Sawtelle, Chandra Turpen, and Edward F Redish. Chemical energy in an introductory physics course for the life sciences. American Journal of Physics, 82(5):403-411, 2014.

[33] Brian O'Shea, Laura Terry, and Walter Benenson. From $\mathrm{f}=$ ma to flying squirrels: Curricular change in an introductory physics course. CBE-Life Sciences Education, 12(2):230-238, 2013.

[34] SGJ Mochrie. Vision and change in introductory physics for the life sciences. American Journal of Physics, 84(7):542-551, 2016.

[35] John R Cameron, James G Skofronick, and Roderick M Grant. Medical physics: physics of the body. Medical Physics Publishing Corporation, 1992. 
[36] Frederick Ross Hallett and Robert Henry Stinson. Physics for the biological sciences. Nelson Canada, 1990.

[37] Jerrold T Bushberg and John M Boone. The essential physics of medical imaging. Lippincott Williams \& Wilkins, 2011.

[38] Russell K Hobbie and Bradley J Roth. Intermediate physics for medicine and biology. Springer Science \& Business Media, 2007.

[39] George B Benedek and Felix MH Villars. Physics with illustrative examples from medicine and biology: mechanics, volume 1. Springer Science \& Business Media, 2000.

[40] Suzanne Amador Kane. Introduction to physics in modern medicine. Taylor \& Francis, 2009.

[41] Philip Nelson. Biological physics. WH Freeman New York, 2004.

[42] Philip R Kesten and David L Tauck. University Physics for the Physical and Life Sciences, volume 2. Macmillan Higher Education, 2012.

[43] Conference on Introductory Physics for the Life Sciences. American Association of Physics Teachers, 2014.

[44] Grace R Van Ness and Ralf Widenhorn. Engaging the community through an undergraduate biomedical physics course. American Journal of Physics, 80(12):1094-1098, 2012.

[45] Elliot Mylott, Ellynne Kutschera, Justin C Dunlap, Warren Christensen, and Ralf Widenhorn. Using biomedically relevant multimedia content in an introductory physics course for life science and pre-health students. Journal of Science Education and Technology, pages 1-10, 2015.

[46] Physics of Biomedicine - Project's YouTube Channel. https://www . youtube.com/user/PhysicsinBiomedicine/.

[47] Chris S Hulleman and Judith M Harackiewicz. Promoting interest and performance in high school science classes. Science (New York, N.Y.), 326:1410-1412, 2009.

[48] Chris S. Hulleman, Amanda M. Durik, Shaun A. Schweigert, and Judith M. Harackiewicz. Task values, achievement goals, and interest: An integrative analysis. Journal of Educational Psychology, 100(2):398-416, 2008.

[49] Akane Zusho, Paul R Pintrich, and Brian Coppola. Skill and will: the role of motivation and cognition in the learning of college chemistry. International Journal of Science Education, 25(9):1081-1094, 2003. 
[50] Suzanne Hidi and K. Ann Renninger. The Four-Phase Model of Interest Development. Educational Psychologist, 41(2):111-127, 2006.

[51] Ann M L Cavallo, Michelle Rozman, and Wendell H Potter. Gender Differences in Learning Constructs, Shifts in Learning Constructs, and Their Relationship to Course Achievement in a Structured Inquiry, Yearlong College Physics Course for Life Science Majors. School Science and Mathematics, 104(6):288, 2004.

[52] Mitchell M Handelsman, William L Briggs, Nora Sullivan, and Annette Towler. A Measure of College Student Course Engagement. The Journal of Educational Research, 98(3):184-192, 2005.

[53] Thomas Koballa. Framework for the affective domain in science education. The Affective Domain in Science Education, pages 3-5, 2014.

[54] Peter Hussler and Lore Hoffmann. An intervention study to enhance girls' interest, self-concept, and achievement in physics classes. Journal of Research in Science Teaching, 39(9):870-888, 2002.

[55] P R Pintrich. A Motivational Science Perspective on the Role of Student Motivation in Learning and Teaching Contexts. Journal of Educational Psychology, 95(4):667-686, 2003.

[56] Almer Abak Gungor, Ali Eryılmaz, and Turgut Fakıoglu. The relationship of freshmen's physics achievement and their related affective characteristics. Journal of Research in Science Teaching, 44(8):1036-1056, 2007.

[57] Catherine H Crouch, Panchompoo Wisittanawat, and K Ann Renninger. Initial Interest, Goals, and Changes in CLASS Scores in Introductory Physics for Life Sciences. AIP Conference Proceedings, 1513(Physics 3):3-6, 2013.

[58] Dawn C. Meredith and Jessica A. Bolker. Rounding off the cow: Challenges and successes in an interdisciplinary physics course for life science students. American Journal of Physics, 80(10):913, 2012.

[59] Gerd Kortemeyer. The challenge of teaching introductory physics to premedical students. The Physics Teacher, 45(9):552-557, 2007.

[60] Wendy K Adams, Katherine K Perkins, Noah S Podolefsky, Michael Dubson, Noah D Finkelstein, and Carl E Wieman. New instrument for measuring student beliefs about physics and learning physics: The colorado learning attitudes about science survey. Physical review special topics-physics education research, 2(1):010101, 2006.

[61] Edward F Redish, Jeffery M Saul, and Richard N Steinberg. Student expectations in introductory physics. American Journal of Physics, 66(3):212-224, 1998. 
[62] Jessica Watkins, Janet E. Coffey, Edward F. Redish, and Todd J. Cooke. Disciplinary authenticity: Enriching the reforms of introductory physics courses for life-science students. Physical Review Special Topics - Physics Education Research, 8(1):1-17, 2012.

[63] Ruskell T., M. Selen, and T. Stelzer. Flipitphysics.

https://www.flipitphysics.com.

[64] Physics of Biomedicine - Laboratory Activities. http://web.pdx.edu/ ralfw/biomedical-physics-labs.html.

[65] Elliot Mylott, Ellynne Kutschera, and Ralf Widenhorn. Bioelectrical impedance analysis as a laboratory activity: At the interface of physics and the body. American Journal of Physics, 82(5):521-528, 2014.

[66] Elliot Mylott, Ryan Klepetka, Justin C Dunlap, and Ralf Widenhorn. An easily assembled laboratory exercise in computed tomography. European Journal of Physics, 32(5):1227, 2011.

[67] Heike Theyßen, Sarah Struzyna, Elliot Mylott, and Ralf Widenhorn. Online physics lab exercisesa binational study on the transfer of teaching resources. International Journal of Science and Mathematics Education, pages 1-19, 2015.

[68] Ellynne Kutschera, Justin C Dunlap, Misti Byrd, Casey Norlin, and Ralf Widenhorn. Pulse oximetry in the physics lab: A colorful alternative to traditional optics curricula. The Physics Teacher, 51(8):495-497, 2013.

[69] Justin C Dunlap, Ellynne Kutschera, Grace R Van Ness, and Ralf Widenhorn. The electrocardiogram as an electronic filter and why ac circuits are important for pre-health physics students. Physics Education, 50(1):81, 2015.

[70] Elliot Mylott, Justin Dunlap, Lester Lampert, and Ralf Widenhorn. Kinesthetic activities for the classroom. The Physics Teacher, 52(9):525-528, 2014

[71] Orjan G Martinsen and Sverre Grimnes. Bioimpedance and bioelectricity basics. Academic press, 2011.

[72] Kenneth R Foster and Henry C Lukaski. Whole-body impedance-what does it measure? The American Journal of Clinical Nutrition, 64(3):388S-396S, 1996.

[73] Antonio Piccoli, Giordano Pastori, et al. Equivalence of information from single versus multiple frequency bioimpedance vector analysis in hemodialysis. Kidney International, 67(1):301-313, 2005.

[74] Antonio Piccoli, Luana Pillon, and Francis Dumler. Impedance vector distribution by sex, race, body mass index, and age in the united states: 
standard reference intervals as bivariate z scores. Nutrition, 18(2):153-167, 2002 .

[75] Ursula G Kyle, Ingvar Bosaeus, Antonio D De Lorenzo, Paul Deurenberg, Marinos Elia, José Manuel Gómez, Berit Lilienthal Heitmann, Luisa Kent-Smith, Jean-Claude Melchior, Matthias Pirlich, et al. Bioelectrical impedance analysis part I: review of principles and methods. Clinical nutrition, 23(5):1226-1243, 2004.

[76] Analog Devices, One Technology Way, PO Box 9106, Norwood, MA 02062-9106, USA. AD8302 Data Sheet, 2002.

[77] Yuxiang Yang, Jue Wang, Gang Yu, Feilong Niu, and Ping He. Design and preliminary evaluation of a portable device for the measurement of bioimpedance spectroscopy. Physiological Measurement, 27(12):1293, 2006.

[78] OMRON Healthcare, INC., 1200 Lakeside Drive, Bannockburn, IL 60015, USA. OMRON HBF-306C Fat Loss Monitor Manual.

[79] KS Cole. Dispersion and absorption in dielectrics. J. Chem. Phys, 9:341, 1941.

[80] More information on the Vernier photogate can be found at http://www. vernier.com/products/sensors/vpg-btd/.

[81] More information on the Vernier rotary motion sensor can be found at http://www . vernier.com/products/sensors/rmv-btd/.

[82] LEE Filters. More information on the congo blue filter can be found at. http://www. leefilters.com/lighting/colour-details.html\#181.

[83] Monika Hüther. Evaluation einer hypermedialen Lernumgebung zum Thema Gasgesetze: eine Studie im Rahmen des Physikpraktikums für Studierende der Medizin. Logos-Verlag, 2005.

[84] Heike Theyßen and Monika Hüther. Evaluation of the hypermedia learning environment physics for medical students within two different settings. In Contributions from Science Education Research, pages 477-487. Springer, 2007.

[85] Heike Theyßen. Students' attitudes towards the hypermedia learning environment physics for medical students. European Journal of Open, Distance and E-Learning, 2006.

[86] J Kirstein. The educational value of "interactive screen experiments": a new representation of experiments with multimedia technology. In Proceedings of the Third International Conference on Science Education Research in the Knowledge Based Society. Thessaloniki: Art of Text, II, pages 468-470, 2001. 
[87] Otto Zietz, Elliot Mylott, and Ralf Widenhorn. Infrared radiography: Modeling x-ray imaging without harmful radiation. The Physics Teacher, 53(1):46-49, 2015.

[88] Results of the analysis of the ekg laboratory activity. http://web.pdx.edu/ ralfw/ekg.html.

[89] Priscilla Laws. Workshop physics: Learning introductory physics by doing it. Change: The Magazine of Higher Learning, 23(4):20-27, 1991.

[90] Panagiotis Pantidos and Stamatis Patapis. Kinesthetic transverse wave demonstration. The Physics Teacher, 43(6):344-345, 2005.

[91] Brooke A Whitworth, Jennifer L Chiu, and Randy L Bell. Kinesthetic investigations in the physics classroom. The Physics Teacher, 52(2):91-93, 2014.

[92] Oregon museum of science and industry - science pub. https://omsi.edu/sciencepub.

[93] American association of physics teachers - 2013 summer conference demo show. https://www.aapt.org/Conferences/sm2013.

[94] Physics of Biomedicine - Multimedia Material. http://web.pdx.edu/ ralfw/multimedia.html.

[95] James S. Walker. Physics with Mastering Physics. Pearson, 4 edition, 2015.

[96] Adrian Madsen, Sarah B McKagan, and Eleanor C Sayre. How physics instruction impacts students beliefs about learning physics: A meta-analysis of 24 studies. Physical Review Special Topics-Physics Education Research, 11(1):010115, 2015.

[97] More information on the DeskCAT by Modus Medical can be found at http://modusqa.com/education/deskcat.

[98] More information of the PhET activities from the University of Colorado Boulder can be found at http://phet.colorado.edu/.

[99] More information on the Ignite Talks can be found on the Ignite website http://www.ignitetalks.io/.

[100] Lisa H. Weasel and Liza Finkel. Deliberative pedagogy in a nonmajors biology course: Active learning that promotes student engagement with science policy and research. Journal of College Science Teaching, 45(4), 2016.

[101] Charles De Leone, Catherine M. Ishikawa, and Robin Marion. Adaptation and implementation of a radically reformed introductory physics course for biological science majors: Assessing success and prospects for future 
implementation. In Physics Education Research Conference 2006, volume 883 of PER Conference, pages 113-116, Syracuse, New York, July 26-27 2006.

[102] Colorado Learning Attitudes about Science Survey.

http://www. colorado.edu/sei/class/.

[103] More einformation about physport can be found at their website. https://www. physport.org/.

[104] Eric Brewe, Adrienne Traxler, Jorge De La Garza, and Laird H. Kramer. Extending positive CLASS results across multiple instructors and multiple classes of Modeling Instruction. Physical Review Special Topics - Physics Education Research, 9(2):1-10, 2013.

[105] Expert reccomendations for use of the effect size can be found on the physport website.

https : //www . physport .org/recommendations/Entry . cfm?ID=93385.

[106] Barry H Cohen and R Brooke Lea. Essentials of statistics for the social and behavioral sciences, volume 3. John Wiley \& Sons, 2004.

[107] David Hestenes, Malcolm Wells, and Gregg Swackhamer. Force concept inventory. The Physics Teacher, 30(3):141-158, 1992.

[108] Ronald K. Thornton and David R. Sokoloff. Assessing student learning of newtons laws: The force and motion conceptual evaluation and the evaluation of active learning laboratory and lecture curricula. American Journal of Physics, 66(4):338-352, 1998.

[109] Ronald Roedel, Samir El-Ghazaly, Teri Reed-Rhoads, and El-Badawy El-Sharawy. The wave concepts inventory - an assessment tool for courses in electromagnetic engineering. Technical Report F2A, November 1998.

[110] Derya Kaltakci-Gurel, Ali Eryilmaz, and Lillian Christie McDermott. Development and application of a four-tier test to assess pre-service physics teachers misconceptions about geometrical optics. Research in Science 8 Technological Education, 35(2):238-260, 2017.

[111] Dennis L Clason and Thomas J Dormody. Analyzing data measured by individual likert-type items. Journal of agricultural education, 35:4, 1994.

[112] Harry N Boone and Deborah A Boone. Analyzing likert data. Journal of extension, 50(2):1-5, 2012.

[113] Mary L McHugh. The chi-square test of independence. Biochemia medica, 23(2):143-149, 2013. 
[114] Zhongzhou Chen, Timothy Stelzer, and Gary Gladding. Using multimedia modules to better prepare students for introductory physics lecture. Phys. Rev. ST Phys. Educ. Res., 6:010108, Jun 2010.

[115] Judith M Harackiewicz, Christopher S Rozek, Chris S Hulleman, and Janet S Hyde. Helping parents to motivate adolescents in mathematics and science: An experimental test of a utility-value intervention. Psychological Science, 23(8):899-906, 2012.

[116] Google analytics can be used to improve assessment of student interactions with the biomedical expert videos. google.com/analytics. 
Appendices 


\section{Appendix A.0. CLASS History at Portland State University}

The Colorado Learning Attitudes about Science Survey (CLASS) has been administered in the algebra-based introductory physics sequence at PSU since 2012. This section summarizes the results of those surveys. The table below shows the schedule of when students were asked to complete the CLASS.

\begin{tabular}{|c|c|c|c|c|c|c|}
\hline \multirow{2}{*}{ Year } & \multicolumn{2}{|c|}{ Fall } & \multicolumn{2}{c|}{ Winter } & \multicolumn{2}{c|}{ Spring } \\
\cline { 2 - 6 } & Start & End & Start & End & Start & End \\
\hline $2012-2013$ & CLASS & CLASS & & & & CLASS \\
\hline $2013-2014$ & CLASS & & & CLASS & & CLASS \\
\hline $2014-2015$ & CLASS & CLASS & & & & CLASS \\
\hline $2015-2016$ & CLASS & CLASS & & CLASS & & CLASS \\
\hline
\end{tabular}

Figure A.1: Schedule of CLASS at PSU. The Start and End columns indicate whether the survey was given at the beginning or the end of the term.

The categories of the CLASS are abbreviated in this section according to the following table. Asterisks in the graphs below denote that the shift was statistically significant, which is defined as a shift greater than twice the standard error.

Table A.1: List of categories in the CLASS and the abbreviations used in this section.

\begin{tabular}{l|l} 
Category & Abbreviation \\
\hline \hline Overall & O \\
All Categories & AC \\
Real World Connection & RWC \\
Personal Interest & PI \\
Sense Making/Effort & SME \\
Conceptual Understanding & CU \\
Applied Conceptual Understanding & ACU \\
Problem Solving General & PSG \\
Problem Solving Sophistication & PSS \\
Problem Solving Confidence & PSC \\
\hline
\end{tabular}




\section{A.1 School Year 2015-2016}

\begin{tabular}{|c|c|c|c|c|c|c|}
\hline \multirow{2}{*}{ Year } & \multicolumn{2}{|c|}{ Fall } & \multicolumn{2}{c|}{ Winter } & \multicolumn{2}{c|}{ Spring } \\
\cline { 2 - 7 } & Start & End & Start & End & Start & End \\
\hline $2012-2013$ & CLASS & CLASS & & & & CLASS \\
\hline $2013-2014$ & CLASS & & & CLASS & & CLASS \\
\hline $2014-2015$ & CLASS & CLASS & & & & CLASS \\
\hline $2015-2016$ & CLASS & CLASS & & CLASS & & CLASS \\
\hline
\end{tabular}

These are the results for both cohorts. Only matched data is included. This data includes both pre-health and non-pre-health students.

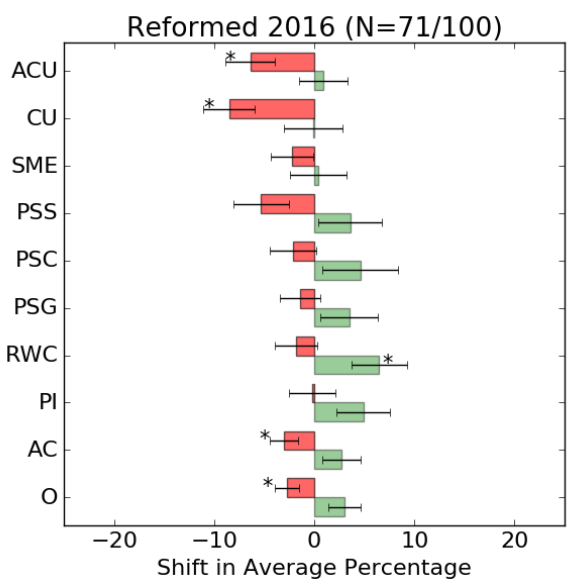

(a)

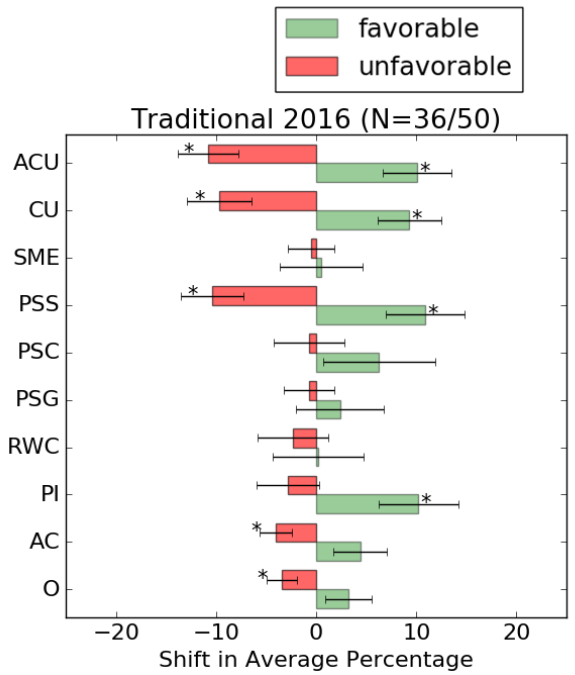

(b)

Table A.2: Shifts in average percent favorable responses from students in the reformed and traditional courses in the spring 2016 term. Asterisks denote statistically significant shifts, which are defined as twice the standard error.

\begin{tabular}{l|llll|llll} 
& \multicolumn{4}{|c|}{ Traditional $(\mathrm{N}=71 / 100)$} & \multicolumn{4}{c}{ Reformed $(\mathrm{N}=36 / 50)$} \\
Cat. & Pre & Post & Shift & StErr & Pre & Post & Shift & StErr \\
\hline \hline O & 57.9 & 60.8 & 3.0 & 1.6 & 52.8 & 56.0 & 3.2 & 2.3 \\
AC & 57.6 & 60.3 & 2.7 & 1.9 & 51.3 & 55.7 & 4.4 & 2.7 \\
PI & 58.9 & 63.8 & 4.9 & 2.7 & 52.3 & 62.5 & $10.2^{*}$ & 4.0 \\
RWC & 68.2 & 74.6 & $6.5^{*}$ & 2.8 & 65.5 & 65.7 & 0.2 & 4.5 \\
PSG & 66.5 & 70.1 & 3.5 & 2.9 & 60.8 & 63.2 & 2.4 & 4.4 \\
PSC & 65.5 & 70.1 & 4.6 & 3.8 & 60.4 & 66.7 & 6.3 & 5.6 \\
PSS & 43.2 & 46.8 & 3.6 & 3.2 & 37.2 & 48.1 & $10.9^{*}$ & 3.9 \\
SME & 64.8 & 65.2 & 0.4 & 2.8 & 55.2 & 55.6 & 0.5 & 4.1 \\
CU & 54.7 & 54.6 & -0.1 & 2.9 & 42.6 & 51.9 & $9.3^{*}$ & 3.2 \\
ACU & 38.8 & 39.7 & 0.9 & 2.4 & 30.8 & 40.9 & $10.1^{*}$ & 3.4 \\
\hline
\end{tabular}




\begin{tabular}{|c|c|c|c|c|c|c|}
\hline \multirow{2}{*}{ Year } & \multicolumn{2}{|c|}{ Fall } & \multicolumn{2}{c|}{ Winter } & \multicolumn{2}{c|}{ Spring } \\
\cline { 2 - 7 } & Start & End & Start & End & Start & End \\
\hline $2012-2013$ & CLASS & CLASS & & & & CLASS \\
\hline $2013-2014$ & CLASS & & & CLASS & & CLASS \\
\hline $2014-2015$ & CLASS & CLASS & & & & CLASS \\
\hline $2015-2016$ & CLASS & CLASS & & CLASS & & CLASS \\
\hline
\end{tabular}

The results below only include pre-health students.

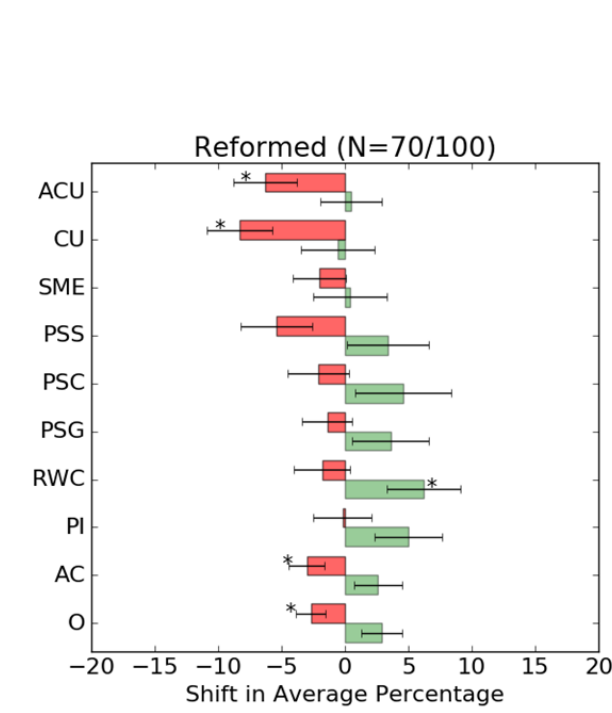

(a)

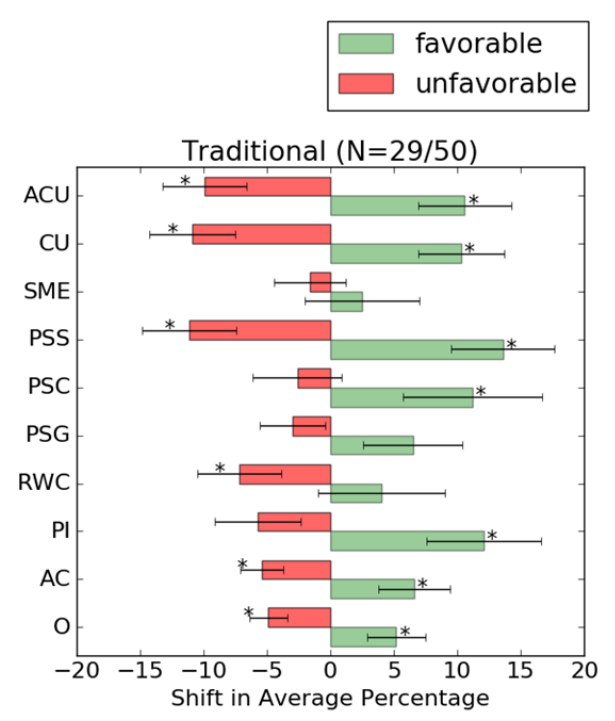

(b)

Figure A.2: Results of the CLASS administered at the end of the winter and spring terms for students in (a) the reformed course and (b) the traditional course. The asterisks denote statistically significant shifts.

Table A.3: Shifts in average percent favorable responses from students in the reformed and traditional courses. Asterisks denote statistically significant shifts, which are defined as twice the standard error. The normalized gain $\langle g\rangle$ for each category is also given.

\begin{tabular}{l|lllll|lllll} 
& \multicolumn{6}{|c|}{ Reformed Course $(\mathrm{N}=70)$} & \multicolumn{5}{|c}{ Traditional Course $(\mathrm{N}=29)$} \\
\hline \hline Cat. & Pre & Post & Shift & StErr & $<g>$ & Pre & Post & Shift & StErr & $<g>$ \\
\hline O & 57.8 & 60.6 & 2.8 & 1.6 & 0.07 & 51.2 & 56.3 & $5.2^{*}$ & 2.3 & 10.6 \\
AC & 57.4 & 60.1 & 2.6 & 1.9 & 0.06 & 49.5 & 56.0 & $6.6^{*}$ & 2.8 & 0.13 \\
PI & 58.6 & 63.6 & 5.0 & 2.7 & 0.12 & 49.4 & 61.5 & $12.1^{*}$ & 4.5 & 0.24 \\
RWC & 68.1 & 74.3 & $6.2^{*}$ & 2.9 & 0.19 & 62.4 & 66.4 & 4.0 & 5.0 & 0.11 \\
PSG & 66.4 & 70.0 & 3.6 & 3.0 & 0.11 & 58.6 & 65.1 & 6.5 & 3.9 & 0.16 \\
PSC & 65.4 & 70.0 & 4.6 & 3.8 & 0.13 & 59.5 & 70.7 & $11.2^{*}$ & 5.5 & 0.28 \\
PSS & 42.9 & 46.3 & 3.4 & 3.2 & 0.06 & 35.9 & 49.4 & $13.6^{*}$ & 4.1 & 0.21 \\
SME & 64.7 & 65.1 & 0.4 & 2.9 & 0.01 & 53.7 & 56.2 & 2.5 & 4.5 & 0.06 \\
CU & 54.8 & 54.2 & -0.6 & 2.9 & -0.01 & 40.8 & 51.1 & $10.3^{*}$ & 3.4 & 0.18 \\
ACU & 38.8 & 39.3 & 0.5 & 2.4 & 0.01 & 30.3 & 40.9 & $10.6^{*}$ & 3.7 & 0.15 \\
\hline
\end{tabular}




\begin{tabular}{|c|c|c|c|c|c|c|}
\hline \multirow{2}{*}{ Year } & \multicolumn{2}{|c|}{ Fall } & \multicolumn{2}{c|}{ Winter } & \multicolumn{2}{c|}{ Spring } \\
\cline { 2 - 6 } & Start & End & Start & End & Start & End \\
\hline $2012-2013$ & CLASS & CLASS & & & & CLASS \\
\hline $2013-2014$ & CLASS & & & CLASS & & CLASS \\
\hline $2014-2015$ & CLASS & CLASS & & & & CLASS \\
\hline $2015-2016$ & CLASS & CLASS & & CLASS & & CLASS \\
\hline
\end{tabular}

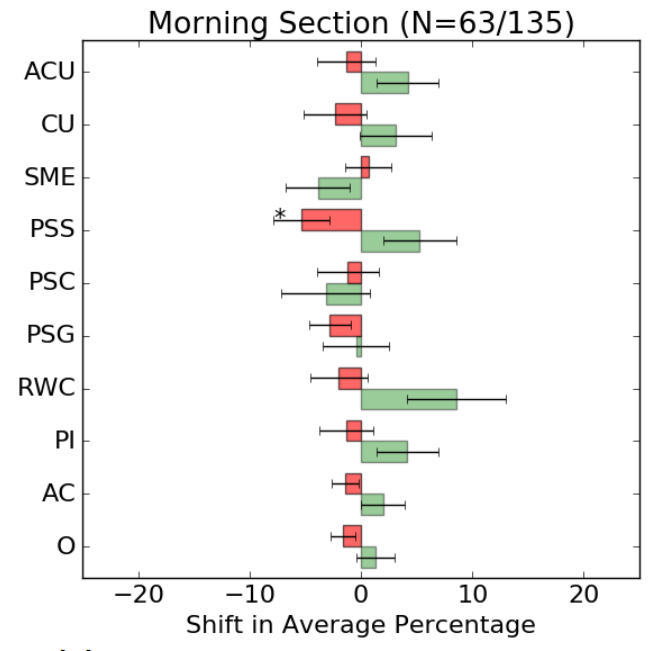

(a)

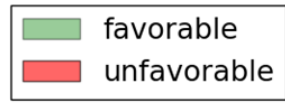

Evening Section $(\mathrm{N}=23 / 48)$

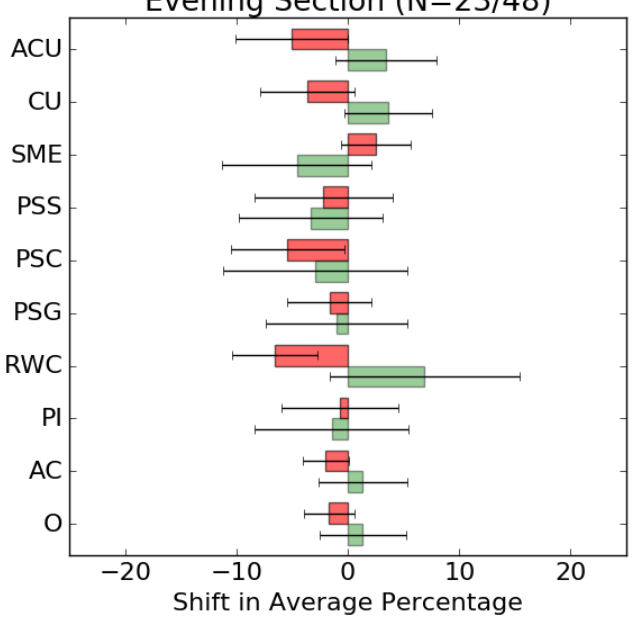

(b)

Table A.4: Shifts in average percent favorable responses from post fall to post winter in the 2015-2016 school year.

\begin{tabular}{l|llll|llll} 
& \multicolumn{4}{|c}{ Morning Section $(\mathrm{N}=63 / 135)$} & \multicolumn{4}{|c}{ Evening Section $(\mathrm{N}=23 / 48)$} \\
Cat. & Pre & Post & Shift & StErr & Pre & Post & Shift & StErr \\
\hline \hline O & 52.47 & 53.78 & 1.31 & 1.73 & 56.94 & 58.29 & 1.34 & 3.87 \\
AC & 51.99 & 53.95 & 1.97 & 1.95 & 55.76 & 57.12 & 1.36 & 4.00 \\
PI & 51.38 & 55.56 & 4.18 & 2.75 & 55.07 & 53.62 & -1.45 & 6.94 \\
RWC & 58.33 & 66.93 & 8.60 & 4.44 & 62.68 & 69.57 & 6.88 & 8.54 \\
PSG & 61.34 & 60.88 & -0.45 & 2.94 & 66.23 & 65.22 & -1.01 & 6.39 \\
PSC & 64.29 & 61.11 & -3.17 & 3.95 & 64.86 & 61.96 & -2.90 & 8.24 \\
PSS & 36.08 & 41.38 & 5.29 & 3.31 & 43.91 & 40.58 & -3.33 & 6.46 \\
SME & 63.42 & 59.56 & -3.85 & 2.89 & 65.84 & 61.28 & -4.55 & 6.71 \\
CU & 46.88 & 50.00 & 3.12 & 3.27 & 51.45 & 55.07 & 3.62 & 3.96 \\
ACU & 33.33 & 37.53 & 4.20 & 2.75 & 36.34 & 39.75 & 3.42 & 4.56 \\
\hline
\end{tabular}




\begin{tabular}{|c|c|c|c|c|c|c|}
\hline \multirow{2}{*}{ Year } & \multicolumn{2}{|c|}{ Fall } & \multicolumn{2}{c|}{ Winter } & \multicolumn{2}{c|}{ Spring } \\
\cline { 2 - 7 } & Start & End & Start & End & Start & End \\
\hline $2012-2013$ & CLASS & CLASS & & & & CLASS \\
\hline $2013-2014$ & CLASS & & & CLASS & & CLASS \\
\hline $2014-2015$ & CLASS & CLASS & & & & CLASS \\
\hline $2015-2016$ & CLASS & CLASS & & CLASS & & CLASS \\
\hline
\end{tabular}

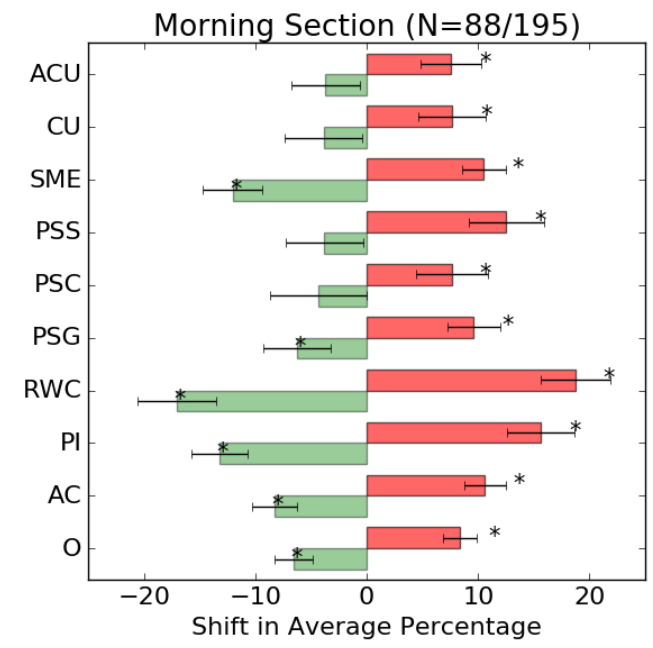

(a)

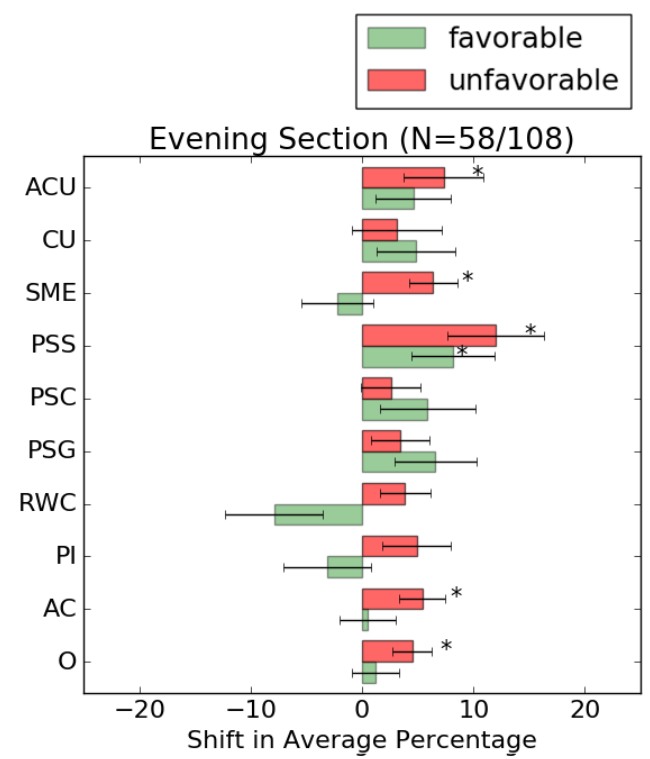

(b)

Table A.5: Shifts in average percent favorable responses from pre fall to post fall in the 2015-2016 school year.

\begin{tabular}{l|llll|llll} 
& \multicolumn{4}{|c}{ Morning Section $(\mathrm{N}=88 / 195)$} & \multicolumn{3}{c}{ Evening Section $(\mathrm{N}=58 / 108)$} \\
Cat. & Pre & Post & Shift & StErr & Pre & Post & Shift & StErr \\
\hline \hline O & 52.62 & 46.07 & -6.56 & 1.75 & 56.43 & 57.66 & 1.23 & 2.13 \\
AC & 52.39 & 44.11 & -8.28 & 2.00 & 56.94 & 57.46 & 0.52 & 2.52 \\
PI & 52.20 & 39.02 & -13.18 & 2.50 & 60.63 & 57.53 & -3.10 & 3.93 \\
RWC & 64.77 & 47.73 & -17.05 & 3.53 & 75.43 & 67.53 & -7.90 & 4.40 \\
PSG & 56.53 & 50.30 & -6.23 & 3.02 & 60.19 & 66.78 & 6.59 & 3.66 \\
PSC & 54.07 & 49.72 & -4.36 & 4.31 & 63.36 & 69.25 & 5.89 & 4.29 \\
PSS & 34.17 & 30.38 & -3.79 & 3.46 & 33.97 & 42.13 & 8.16 & 3.70 \\
SME & 67.86 & 55.82 & -12.04 & 2.66 & 70.65 & 68.43 & -2.22 & 3.19 \\
CU & 47.08 & 43.22 & -3.86 & 3.45 & 44.02 & 48.85 & 4.83 & 3.50 \\
ACU & 33.88 & 30.19 & -3.69 & 3.07 & 32.22 & 36.82 & 4.60 & 3.39 \\
\hline
\end{tabular}




\section{A.1.1 Full Year Trends}

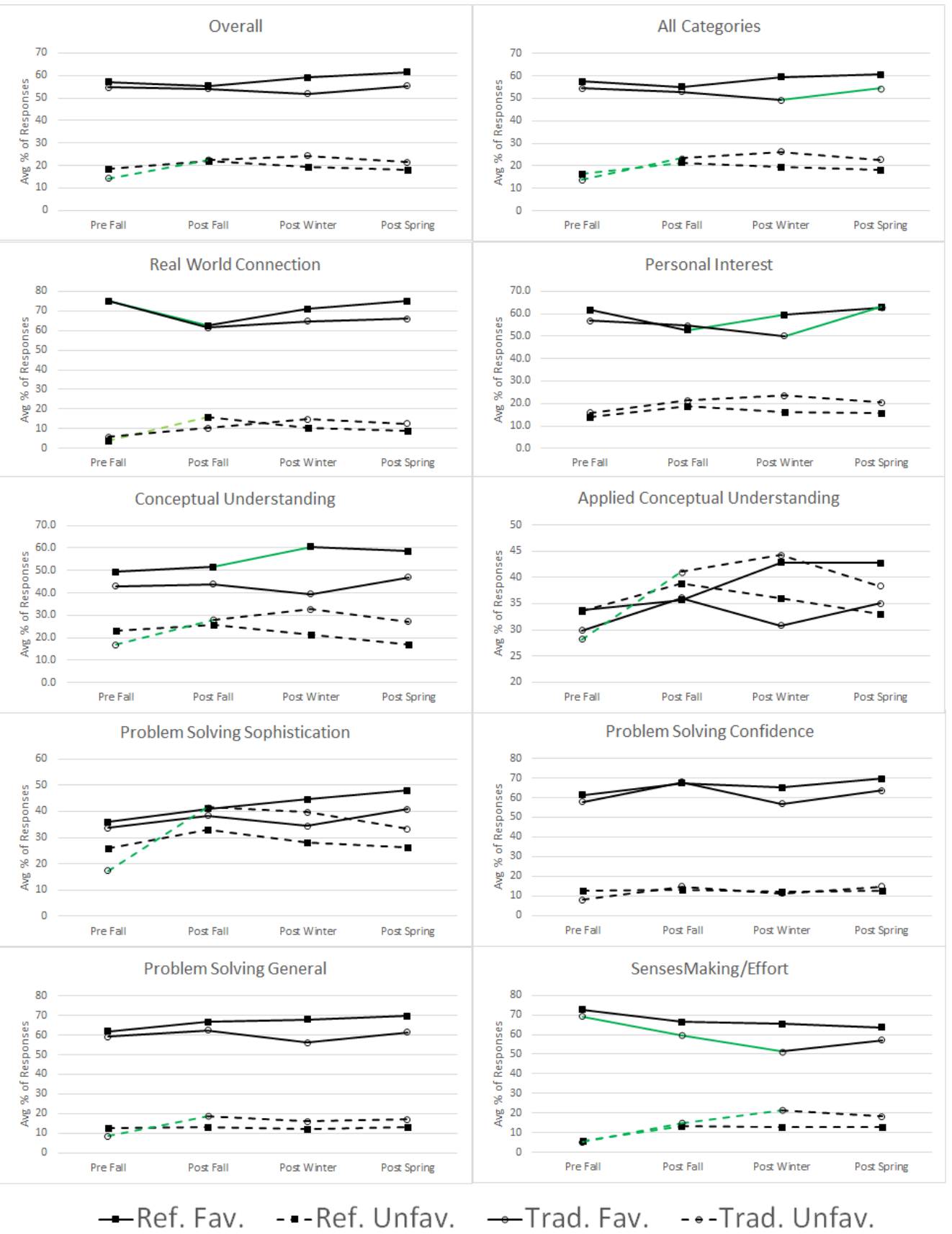

Figure A.3: CLASS results for the full academic year from students in the reformed (black squares) and traditional (empty circles) courses. 46 students from the reformed course and 22 students from the traditional course responded to all four surveys. Green lines represent significant shifts. 


\section{A.2 School Year 2014-2015}

\begin{tabular}{|c|c|c|c|c|c|c|}
\hline \multirow{2}{*}{ Year } & \multicolumn{2}{|c|}{ Fall } & \multicolumn{2}{c|}{ Winter } & \multicolumn{2}{c|}{ Spring } \\
\cline { 2 - 7 } & Start & End & Start & End & Start & End \\
\hline $2012-2013$ & CLASS & CLASS & & & & CLASS \\
\hline $2013-2014$ & CLASS & & & CLASS & & CLASS \\
\hline $2014-2015$ & CLASS & CLASS & & & & CLASS \\
\hline $2015-2016$ & CLASS & CLASS & & CLASS & & CLASS \\
\hline
\end{tabular}

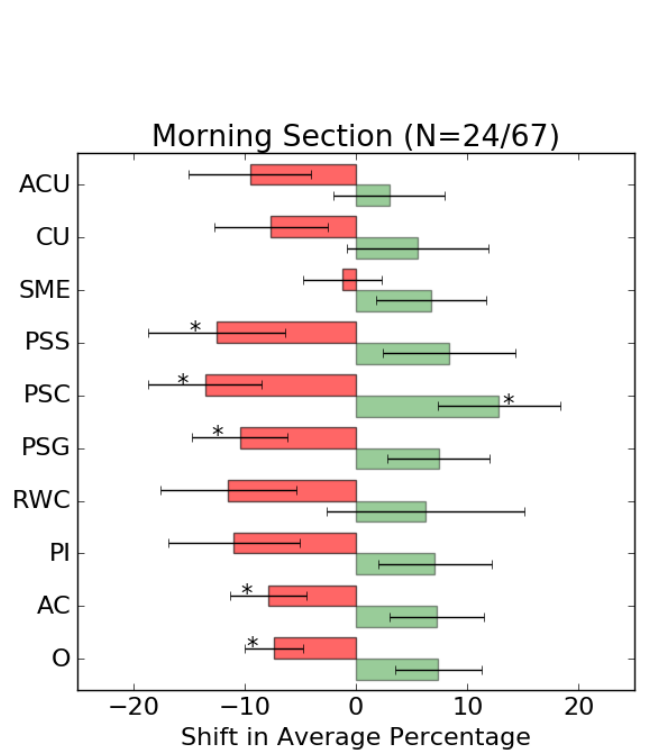

(a)

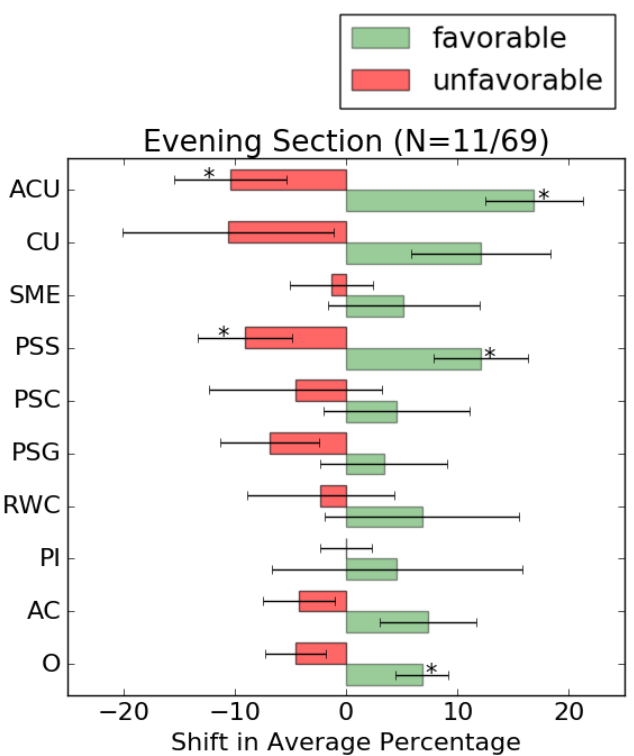

(b)

Figure A.4: Results of the CLASS administered at the end and beginning of the fall term for students in (a) the morning course and (b) the evening course. The asterisks denote statistically significant shifts.

Table A.6: Shifts in average percent favorable responses from post fall to post spring for the 2014-2015 school year.

\begin{tabular}{l|llll|llll} 
& \multicolumn{3}{|c}{ Morning Section $(\mathrm{N}=24 / 67)$} & \multicolumn{4}{|c}{ Evening Section $(\mathrm{N}=11 / 69)$} \\
Cat. & Pre & Post & Shift & StErr & Pre & Post & Shift & StErr \\
\hline \hline O & 49.29 & 56.69 & 7.40 & 3.89 & 58.33 & 65.15 & 6.82 & 2.36 \\
AC & 47.90 & 55.14 & 7.24 & 4.26 & 57.34 & 64.69 & 7.34 & 4.31 \\
PI & 45.00 & 52.08 & 7.08 & 5.09 & 62.12 & 66.67 & 4.55 & 11.25 \\
RWC & 58.33 & 64.58 & 6.25 & 8.89 & 79.55 & 86.36 & 6.82 & 8.70 \\
PSG & 51.04 & 58.48 & 7.44 & 4.59 & 62.50 & 65.91 & 3.41 & 5.71 \\
PSC & 45.83 & 58.68 & 12.85 & 5.49 & 56.82 & 61.36 & 4.55 & 6.57 \\
PSS & 36.81 & 45.14 & 8.33 & 5.96 & 34.85 & 46.97 & 12.12 & 4.22 \\
SME & 53.97 & 60.71 & 6.75 & 4.92 & 66.23 & 71.43 & 5.19 & 6.76 \\
CU & 50.00 & 55.56 & 5.56 & 6.38 & 45.45 & 57.58 & 12.12 & 6.26 \\
ACU & 39.88 & 42.86 & 2.98 & 4.99 & 28.57 & 45.45 & 16.88 & 4.43 \\
\hline
\end{tabular}




\begin{tabular}{|c|c|c|c|c|c|c|}
\hline \multirow{2}{*}{ Year } & \multicolumn{2}{|c|}{ Fall } & \multicolumn{2}{c|}{ Winter } & \multicolumn{2}{c|}{ Spring } \\
\cline { 2 - 7 } & Start & End & Start & End & Start & End \\
\hline $2012-2013$ & CLASS & CLASS & & & & CLASS \\
\hline $2013-2014$ & CLASS & & & CLASS & & CLASS \\
\hline $2014-2015$ & CLASS & CLASS & & & & CLASS \\
\hline $2015-2016$ & CLASS & CLASS & & CLASS & & CLASS \\
\hline
\end{tabular}

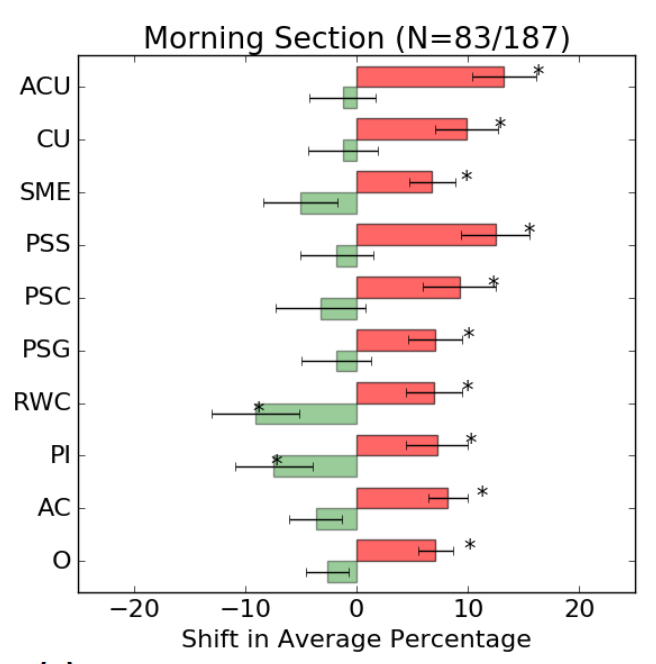

(a)
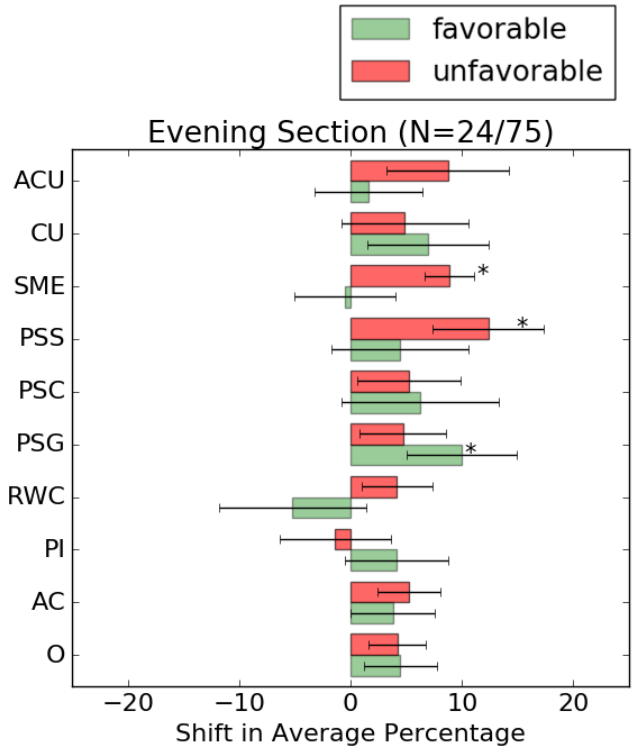

(b)

Table A.7: Shifts in average percent favorable responses from pre fall to post fall for the 2014-2015 school year.

\begin{tabular}{l|llll|llll} 
& \multicolumn{4}{|c}{ Morning Section (N=83/187) } & \multicolumn{4}{|c}{ Evening Section $(\mathrm{N}=24 / 75)$} \\
Cat. & Pre & Post & Shift & StErr & Pre & Post & Shift & StErr \\
\hline \hline O & 57.99 & 55.34 & -2.65 & 1.93 & 54.91 & 59.38 & 4.46 & 3.30 \\
AC & 57.69 & 54.03 & -3.67 & 2.33 & 54.52 & 58.33 & 3.81 & 3.77 \\
PI & 58.07 & 50.64 & -7.43 & 3.48 & 55.56 & 59.72 & 4.17 & 4.63 \\
RWC & 71.08 & 62.05 & -9.04 & 3.92 & 73.96 & 68.75 & -5.21 & 6.58 \\
PSG & 63.58 & 61.75 & -1.83 & 3.14 & 56.18 & 66.15 & 9.97 & 4.94 \\
PSC & 63.45 & 60.24 & -3.21 & 4.04 & 56.25 & 62.50 & 6.25 & 7.10 \\
PSS & 42.09 & 40.32 & -1.77 & 3.28 & 40.00 & 44.44 & 4.44 & 6.13 \\
SME & 67.81 & 62.77 & -5.05 & 3.31 & 68.35 & 67.86 & -0.50 & 4.49 \\
CU & 49.92 & 48.67 & -1.24 & 3.12 & 45.14 & 52.08 & 6.94 & 5.46 \\
ACU & 39.19 & 37.92 & -1.26 & 2.96 & 35.91 & 37.50 & 1.59 & 4.82 \\
\hline
\end{tabular}




\section{A.3 School Year 2013-2014}

\begin{tabular}{|c|c|c|c|c|c|c|}
\hline \multirow{2}{*}{ Year } & \multicolumn{2}{|c|}{ Fall } & \multicolumn{2}{c|}{ Winter } & \multicolumn{2}{c|}{ Spring } \\
\cline { 2 - 7 } & Start & End & Start & End & Start & End \\
\hline $2012-2013$ & CLASS & CLASS & & & & CLASS \\
\hline $2013-2014$ & CLASS & & & CLASS & & CLASS \\
\hline $2014-2015$ & CLASS & CLASS & & & & CLASS \\
\hline $2015-2016$ & CLASS & CLASS & & CLASS & & CLASS \\
\hline
\end{tabular}

This year the reformed course was offered in the evening section.

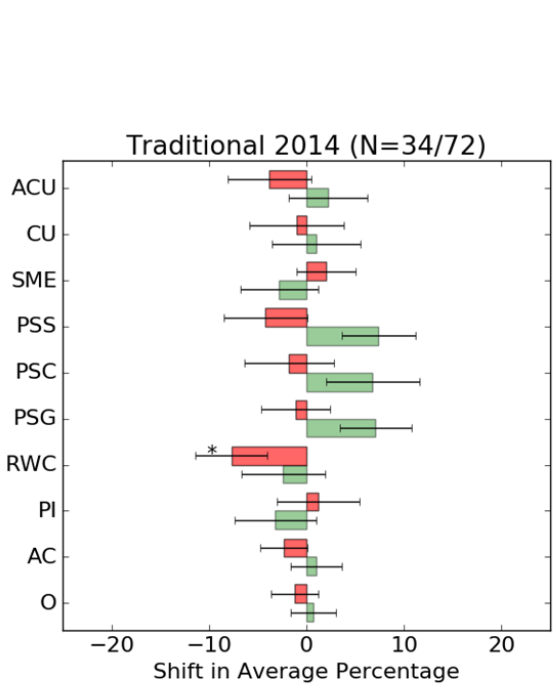

(a)

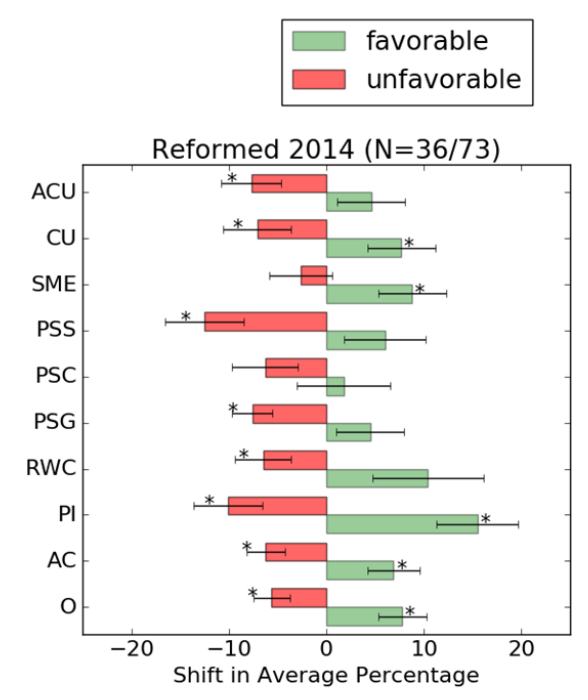

(b)

Figure A.5: Results of the CLASS administered for (a) the morning (traditional) course and (b) the evening (reformed) course.

Table A.8: Shifts in average percent favorable responses from post winter to post spring for the 2013-2014 school year.

\begin{tabular}{l|llll|llll} 
& \multicolumn{4}{|c|}{ Morning Section $(\mathrm{N}=34 / 72)$} & \multicolumn{4}{c}{ Evening Section $(\mathrm{N}=36 / 73)$} \\
Cat. & Pre & Post & Shift & StErr & Pre & Post & Shift & StErr \\
\hline \hline O & 55.9 & 63.7 & 7.8 & 2.5 & 48.2 & 48.9 & 0.7 & 2.3 \\
AC & 55.4 & 62.4 & 6.9 & 2.7 & 45.8 & 46.8 & 1.0 & 2.6 \\
PI & 50.0 & 65.5 & 15.5 & 4.2 & 48.8 & 45.6 & -3.2 & 4.2 \\
RWC & 62.8 & 73.2 & 10.4 & 5.7 & 58.9 & 56.5 & -2.4 & 4.3 \\
PSG & 67.0 & 71.4 & 4.5 & 3.5 & 49.1 & 56.3 & 7.1 & 3.7 \\
PSC & 67.0 & 68.8 & 1.8 & 4.8 & 50.9 & 57.7 & 6.8 & 4.8 \\
PSS & 41.7 & 47.6 & 6.0 & 4.2 & 27.4 & 34.8 & 7.4 & 3.8 \\
SME & 63.7 & 72.4 & 8.8 & 3.5 & 54.8 & 52.0 & -2.8 & 4.0 \\
CU & 47.6 & 55.4 & 7.7 & 3.5 & 37.5 & 38.5 & 1.0 & 4.5 \\
ACU & 34.2 & 38.8 & 4.6 & 3.5 & 27.6 & 29.8 & 2.2 & 4.0 \\
\hline
\end{tabular}




\begin{tabular}{|c|c|c|c|c|c|c|}
\hline \multirow{2}{*}{ Year } & \multicolumn{2}{|c|}{ Fall } & \multicolumn{2}{c|}{ Winter } & \multicolumn{2}{c|}{ Spring } \\
\cline { 2 - 7 } & Start & End & Start & End & Start & End \\
\hline $2012-2013$ & CLASS & CLASS & & & & CLASS \\
\hline $2013-2014$ & CLASS & & & CLASS & & CLASS \\
\hline $2014-2015$ & CLASS & CLASS & & & & CLASS \\
\hline $2015-2016$ & CLASS & CLASS & & CLASS & & CLASS \\
\hline
\end{tabular}

This section only contains data for student who would go on to be in either the evening (reformed) or morning (traditional) version of the spring course. The data is therefore separated by which course they took spring term.

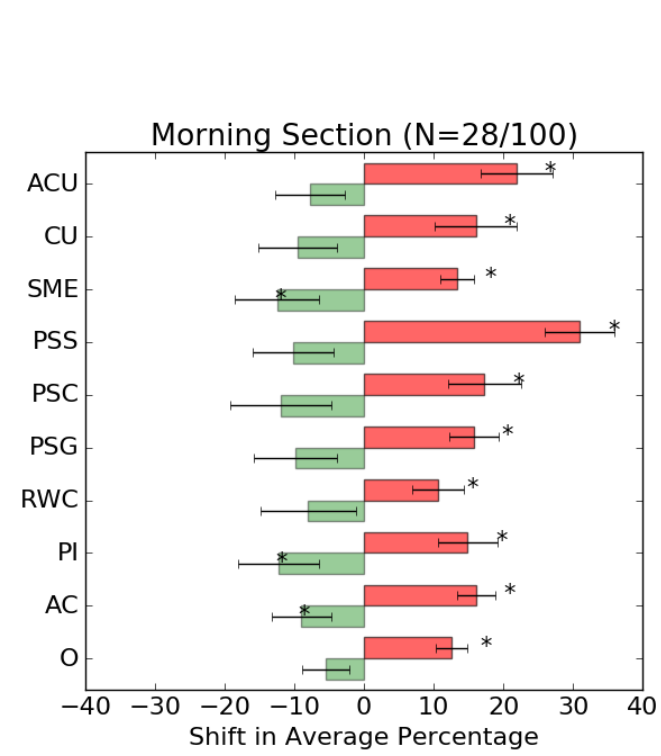

(a)

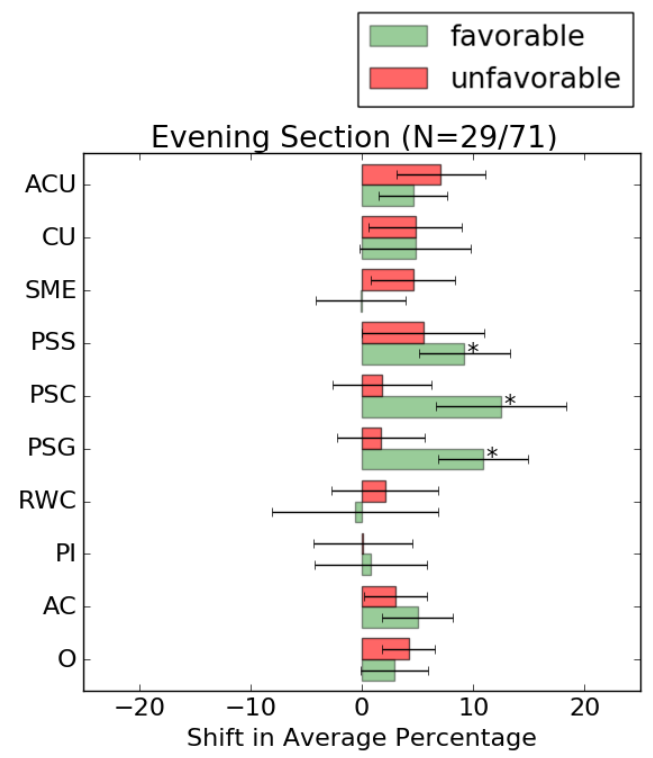

(b)

Figure A.6: Results of the CLASS for students who go on to be in (a) the traditional course and (b) the reformed spring 2014 courses.

Table A.9: Shifts in average percent favorable responses from pre fall to post winter for the 2013-2014 school year.

\begin{tabular}{l|llll|llll} 
& \multicolumn{4}{|c|}{ Traditional Section $(\mathrm{N}=28 / 100)$} & \multicolumn{4}{c}{ Reformed Section $(\mathrm{N}=29 / 71)$} \\
Cat. & Pre & Post & Shift & StErr & Pre & Post & Shift & StErr \\
\hline \hline O & 54.9 & 49.4 & -5.5 & 3.4 & 55.5 & 58.4 & 2.9 & 3.0 \\
AC & 55.6 & 46.7 & -9.0 & 4.3 & 53.7 & 58.7 & 5.0 & 3.2 \\
PI & 62.9 & 50.6 & -12.3 & 5.8 & 55.6 & 56.4 & 0.8 & 5.0 \\
RWC & 70.5 & 62.5 & -8.0 & 6.8 & 69.6 & 69.0 & -0.6 & 7.5 \\
PSG & 58.8 & 49.0 & -9.8 & 6.0 & 58.6 & 69.5 & 10.9 & 4.0 \\
PSC & 63.1 & 51.2 & -11.9 & 7.3 & 57.1 & 69.6 & 12.5 & 5.8 \\
PSS & 39.9 & 29.8 & -10.1 & 5.8 & 36.3 & 45.5 & 9.2 & 4.1 \\
SME & 65.8 & 53.3 & -12.5 & 6.1 & 64.8 & 64.7 & -0.1 & 4.0 \\
CU & 45.2 & 35.7 & -9.5 & 5.6 & 46.4 & 51.2 & 4.8 & 5.0 \\
ACU & 36.2 & 28.6 & -7.7 & 5.0 & 34.2 & 38.8 & 4.6 & 3.1 \\
\hline
\end{tabular}




\section{A.4 School Year 2012-2013}

\begin{tabular}{|c|c|c|c|c|c|c|}
\hline \multirow{2}{*}{ Year } & \multicolumn{2}{|c|}{ Fall } & \multicolumn{2}{c|}{ Winter } & \multicolumn{2}{c|}{ Spring } \\
\cline { 2 - 7 } & Start & End & Start & End & Start & End \\
\hline $2012-2013$ & CLASS & CLASS & & & & CLASS \\
\hline $2013-2014$ & CLASS & & & CLASS & & CLASS \\
\hline $2014-2015$ & CLASS & CLASS & & & & CLASS \\
\hline $2015-2016$ & CLASS & CLASS & & CLASS & & CLASS \\
\hline
\end{tabular}

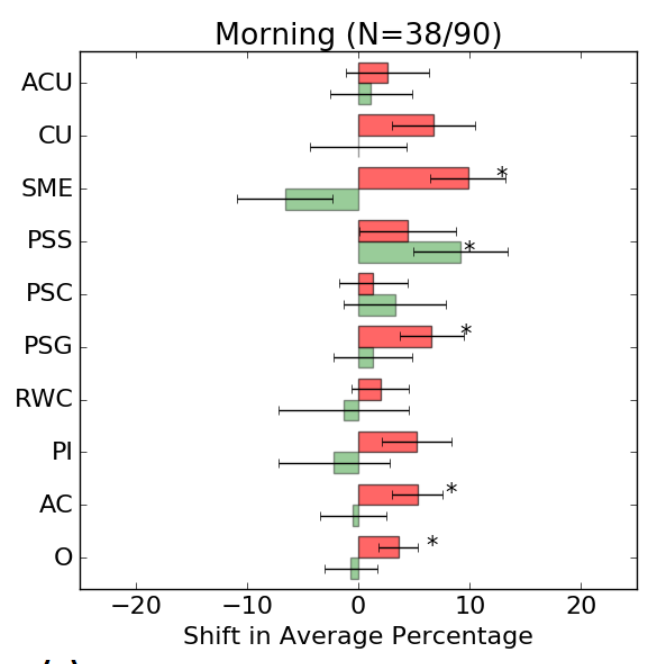

(a)

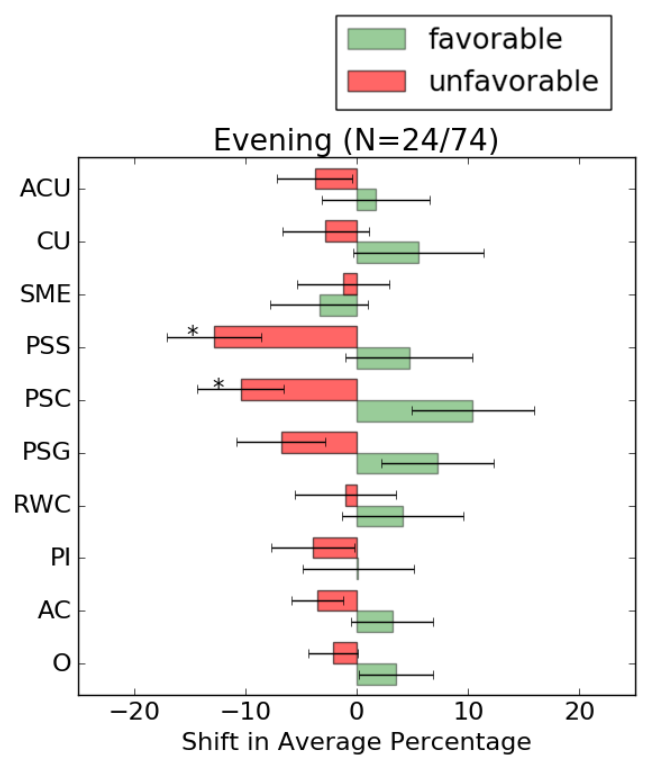

(b)

Table A.10: Shifts in average percent favorable responses fro post fall to post spring for the 2012-2013 school year.

\begin{tabular}{l|llll|llll} 
& \multicolumn{4}{|c|}{ Morning $(\mathrm{N}=38 / 90)$} & \multicolumn{5}{|c}{ Evening $(\mathrm{N}=24 / 74)$} \\
Cat. & Pre & Post & Shift & StErr & Pre & Post & Shift & StErr \\
\hline \hline O & 62.18 & 61.49 & -0.69 & 2.38 & 61.61 & 65.17 & 3.56 & 3.33 \\
AC & 61.50 & 61.04 & -0.46 & 2.97 & 61.42 & 64.60 & 3.18 & 3.67 \\
PI & 59.21 & 57.02 & -2.19 & 4.97 & 68.75 & 68.89 & 0.14 & 5.01 \\
RWC & 70.39 & 69.08 & -1.32 & 5.88 & 75.00 & 79.17 & 4.17 & 5.45 \\
PSG & 68.09 & 69.41 & 1.32 & 3.51 & 62.50 & 69.79 & 7.29 & 5.05 \\
PSC & 67.11 & 70.39 & 3.29 & 4.57 & 61.46 & 71.88 & 10.42 & 5.50 \\
PSS & 46.05 & 55.26 & 9.21 & 4.24 & 50.14 & 54.86 & 4.72 & 5.69 \\
SME & 70.30 & 63.72 & -6.58 & 4.27 & 72.22 & 68.85 & -3.37 & 4.38 \\
CU & 60.96 & 60.96 & 0.00 & 4.37 & 55.56 & 61.11 & 5.56 & 5.87 \\
ACU & 48.50 & 49.62 & 1.13 & 3.70 & 45.93 & 47.62 & 1.69 & 4.85 \\
\hline
\end{tabular}




\begin{tabular}{|c|c|c|c|c|c|c|}
\hline \multirow{2}{*}{ Year } & \multicolumn{2}{|c|}{ Fall } & \multicolumn{2}{c|}{ Winter } & \multicolumn{2}{c|}{ Spring } \\
\cline { 2 - 7 } & Start & End & Start & End & Start & End \\
\hline $2012-2013$ & CLASS & CLASS & & & & CLASS \\
\hline $2013-2014$ & CLASS & & & CLASS & & CLASS \\
\hline $2014-2015$ & CLASS & CLASS & & & & CLASS \\
\hline $2015-2016$ & CLASS & CLASS & & CLASS & & CLASS \\
\hline
\end{tabular}

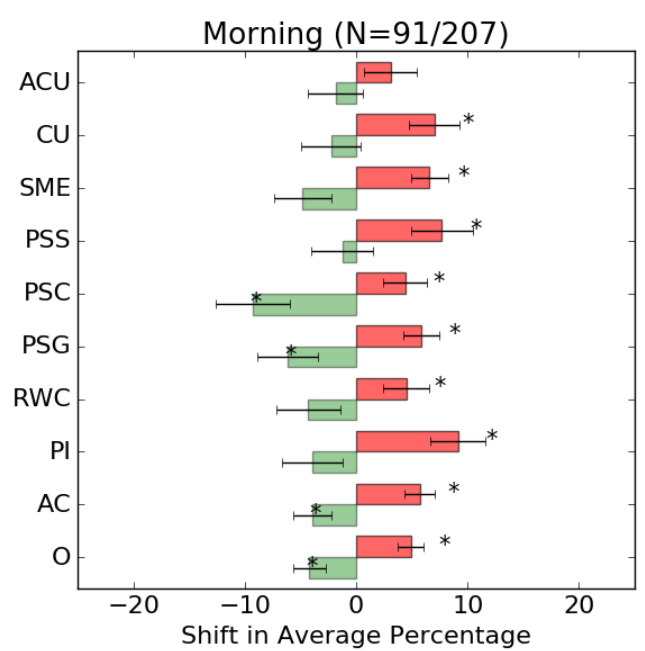

(a)

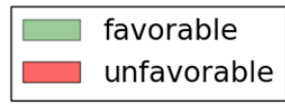

Evening ( $\mathrm{N}=32 / 113)$

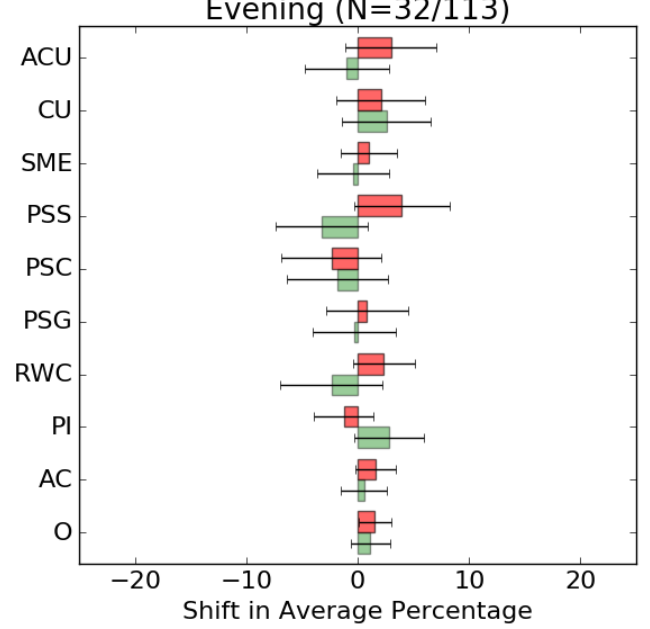

(b)

Table A.11: Shifts in average percent favorable responses from pre fall to post all for the 2012-2013 school year.

\begin{tabular}{l|llll|llll} 
& \multicolumn{4}{|c|}{ Morning $(\mathrm{N}=91 / 207)$} & \multicolumn{4}{|c}{ Evening $(\mathrm{N}=32 / 113)$} \\
Cat. & Pre & Post & Shift & StErr & Pre & Post & Shift & StErr \\
\hline \hline O & 64.00 & 59.79 & -4.21 & 1.46 & 59.17 & 60.32 & 1.14 & 1.76 \\
AC & 63.56 & 59.65 & -3.91 & 1.74 & 59.57 & 60.13 & 0.56 & 2.08 \\
PI & 61.25 & 57.31 & -3.94 & 2.72 & 60.52 & 63.33 & 2.81 & 3.13 \\
RWC & 73.99 & 69.69 & -4.30 & 2.90 & 67.19 & 64.84 & -2.34 & 4.60 \\
PSG & 69.47 & 63.30 & -6.16 & 2.70 & 64.62 & 64.29 & -0.33 & 3.71 \\
PSC & 69.96 & 60.71 & -9.25 & 3.33 & 63.54 & 61.72 & -1.82 & 4.51 \\
PSS & 47.51 & 46.26 & -1.25 & 2.74 & 52.71 & 49.48 & -3.23 & 4.13 \\
SME & 72.76 & 67.97 & -4.79 & 2.57 & 69.87 & 69.49 & -0.37 & 3.23 \\
CU & 60.44 & 58.21 & -2.23 & 2.66 & 57.29 & 59.90 & 2.60 & 3.97 \\
ACU & 49.45 & 47.59 & -1.86 & 2.43 & 46.50 & 45.54 & -0.97 & 3.78 \\
\hline
\end{tabular}




\section{Appendix B.0. Survey Questions}

This section contains all the survey questions that were given to students in the algebra-based introductory physics courses in the 2015-2016 school year.

\section{B.1 Pre Fall}

The pre fall quiz was mostly composed of the 42 CLASS Questions. There were other demographic questions asking about age, major, and courses taken. All of these questions were reformulated in later surveys.

\section{B.2 Post Fall}

(Parentheses indicate that type of question: $\mathrm{MC}=$ multiple choice, $\mathrm{OE}=$ open ended, Likert $=5$ point scale, $\mathrm{Y} / \mathrm{N}=$ yes or no.)

1. 42 CLASS Questions

2. Did you take one of the physics workshops in Fall term? (Y/N)

3. What HIGH SCHOOL physics classes have you completed? (OE)

4. What COLLEGE physics classes have you completed prior to this course? (OE)

5. Please rate how much time in class you think should be spent on the following items. (MC)

(a) Problem Solving/Examples

(b) Lectures

(c) Group Discussion

6. How much time did you spend per week outside of class on the following? $(\mathrm{MC})$

(a) Reading the textbook 
(b) Doing homework problems

(c) Other course related activities

7. Please list any other course related activities you did this term outside of class. (OE)

8. On average, how much time did the spend preparing for each mid term or final exam? (MC)

9. Please indicate below which courses you have completed. (MC)
(a) Fundamental Biology
(b) Biology for Allied Health
(c) Principles of Biology
(d) Anatomy and Physiology
(e) General Chemistry
(f) Organic Chemistry

10. Are you planning to take or have you already taken any of the following exams? (MC)
(a) MCAT
(b) GRE
(c) DAT
(d) PCAT
(e) OAT

11. Is this course required for your major or future graduate program? (Y/N)

12. Do you think this course should be required for your major or future graduate program? $(\mathrm{Y} / \mathrm{N})$

13. Please explain your answer to the previous question. (OE)

14. What is your planned profession after college? (OE)

15. The CONCEPTS I learned in this course will be useful for my future profession/career. (Likert)

16. Please explain your answer to the previous question. (OE)

17. The PROBLEM SOLVING SKILLS I learned in this course will be useful for my future profession/career. (Likert) 
18. Please explain your answer to the previous question. (OE)

19. Specifically, what skills or concepts learned in the course do you think will be the most useful in your future profession/career? (OE)

20. I enjoy discussing topics covered in this course outside of class. (Likert)

21. If you had the option to take either a traditional introductory physics course or an introductory physics course that focuses on the applications of physics in the health/life sciences, which would you choose? (MC)

22. Please explain your answer to the previous question. (OE)

\section{B.3 Post Winter}

The post-winter survey consists of approximately 50 questions including 42 from the CLASS. There are only three long form questions, so students should be able to complete the survey in less than 15 minutes.

There are two primary reasons for administering the CLASS post-winter rather than pre-spring. Firstly, this is the procedure we followed when assessing the 2014 course, so the comparison will be cleaner. The second reason is that the CLASS is a significantly large assessment tool. By including it in winter, we can reduce the size of the assessments in spring and hopefully avoid survey fatigue.

It is important to include items 2 and 3 on both the post-winter and post-spring surveys. We want to analyze whether students spend more time on the reformed course than the traditional course. By having the self-reported data from fall, winter, and spring term, we can better assess shifts than with a single question such as "Compared to previous terms, how much time did you spend working on the course?"

Previous CLASS results at PSU have shown that students in the evening section have more favorable results than students in the morning section. We included items 4-5 to assess the composition of students in the morning or evening section. This will help us determine possible causes of the morning/evening effect and whether that effect will impact the CLASS results from the reformed course.

Finally, items 6-10 will be included in winter and spring for comparison to students' answers in fall. This is used as a rough indicator of students' interest in the course material as well as their perception of the usefulness of the course.

(Parentheses indicate that type of question: $\mathrm{OE}=$ open ended, Likert $=5$ point scale, $\mathrm{Y} / \mathrm{N}=$ yes or no.)

1. 42 CLASS Questions 
2. How much time outside of class do you spend per week on the following? (scale below)
(a) Reading the text
(b) Doing homework problems
(c) Other course related activities
- Less than 30 minutes
- 30 min.-1 hour
- 1-2 hours
- 2-4 hours
- 4-6 hours
- 6-8 hours
- More than 8 hours

3. Please list any other course related activities you did this term outside of class. (OE)

4. On average, how much time did you the spend preparing for each mid-term or final exam? (scale below)

- less than 1 hour

- 1-2 hours

- 2-4 hours

- 4-6 hours

- 6-8 hours

- 8-12 hours

- 12-16 hours

- 16-20 hours

- More than 20 hours

5. Section Option

(a) Which section of PH 203 are you taking? (Morning/Evening)

(b) Please explain why you chose to enroll in this section rather than the other? (OE)

6. Employment 
(a) Are you currently employed? (Y/N)

(b) If yes, how many hours per week do you work on average?

- $0-10$

- $10-20$

- 20-30

- $30-40$

- Over 40

(c) Do you consider your current employment to be an important part of your future career plans? (Likert scale)

7. I enjoy discussing topics covered in this course outside of class. (Likert)

8. Is this course required for your major or future graduate program? $(\mathrm{Y} / \mathrm{N})$

9. Do you think this course should be required for your major or future graduate program? (Likert)

10. Please explain your answer to the previous question. (OE)

11. The concepts I learned in this course will be valuable in my future career. (Likert)

12. The problem solving strategies I learned in this course will be valuable in my future career. (Likert)

\section{B.4 Pre Spring}

These items will be asked in both the pre-spring and post-spring surveys. The pre-spring survey is very short, consisting of less than 15 questions. This should help us avoid survey fatigue.

These questions in item 1 expand on the personal interest (PI) and real world connection (RWC) sections of the CLASS. Specifically, we intend to explore whether students interpret phrases like "everyday life" or "the real world" as including their career related experience. The new items are versions of the CLASS items, rephrased to highlight students' future careers. 
Table B.1: Statements from the Personal Interest and Real World Connection categories of the CLASS.

\begin{tabular}{|l|l|r|r|}
\hline No. & Question & RWC & PI \\
\hline 3. & I think about the physics I experience in everyday life. & & X \\
11. & I am not satisfied until I understand why something works the way it does. \\
14. & I study physics to learn knowledge that will be useful in & X \\
& my life outside of school. & \\
25. & I enjoy solving physics problems. & $\mathrm{X}$ \\
28. & Learning physics changes my ideas about how the world works. \\
30. & Reasoning skills used to understand physics can be helpful to me & $\mathrm{X}$ \\
in my everyday life. & $\mathrm{X}$ \\
35. & The subject of physics has little relation to what I experience & $\mathrm{X}$ \\
37. & $\begin{array}{l}\text { in the real world. } \\
\text { To understand physics, I sometimes think about my personal experiences }\end{array}$ & $\mathrm{X}$ \\
\hline
\end{tabular}

The questions in item 2 probe students' views about the importance of physics in biomedicine. Question 2(a) specifically asks about the relationship of physics to biomedical technology, which is the focus of the reformed course. Question 2(b) is more general, asking students to define the relationship between physics and medicine themselves. By asking these questions to students in both courses, we should be able to see whether the reformed course has an impact on these attitudes.

Finally, item 3 should give us an insight into why students are taking the course and what their expectations are.

1. Extended CLASS related questions (Question number of original CLASS question)

(a) What is your intended profession after college? (OE)

(b) I think about the physics that is involved in my future career. (Likert) (CLASS: 3)

(c) When I learn about technology that is used in my future career, I am not satisfied until I understand why it works the way it does. (Likert) (CLASS: 11)

(d) I study physics to learn knowledge that will be useful in my future career. (Likert) (CLASS: 14)

(e) I enjoy solving physics problems that are relevant to my future career. (Likert) (CLASS: 25)

(f) Reasoning skills used to understand physics can be helpful to me in my future career. (Likert) (CLASS: 30) 
(g) The subject of physics has little relation to what I will experience in my future career. (Likert) (CLASS: 35)

2. Medically related questions

(a) It is useful for biomedical professionals to understand the basic physics principles of the instrumentation they use in their work? (Likert)

(b) What do you view as the relationship between physics and medicine? $(\mathrm{OE})$

3. Why are you taking PH 203? What do you hope to get out of taking the course, and is there anything you are particularly interested in learning about this term? (OE)

\section{B.5 Post Spring}

These questions in items 1-5 will be given to both the traditional and reformed courses in the week before the final exams. The post-spring survey consists of the CLASS and the additional attitudinal questions given in the pre spring survey (items 2-3) as well as some questions that were given to students at the end of fall and winter term (item 4). The questions in item 6 will only be given to students in the reformed course. These questions probe students views about the usefulness of the biomedical focus (questions 6(a) and (b)), expert interviews (questions 6(c) and (d)), and the course overall (questions 6(e) and (f)).

1. 42 CLASS Questions

2. Extended CLASS related questions (Same as in the pre-spring survey)

(a) What is your intended profession after college? (OE)

(b) I think about the physics that is involved in my future career. (Likert) (CLASS: 3)

(c) When I learn about technology that is used in my future career, I am not satisfied until I understand why it works the way it does. (Likert) (CLASS: 11)

(d) I study physics to learn knowledge that will be useful in my future career. (Likert) (CLASS: 14)

(e) I enjoy solving physics problems that are relevant to my future career. (Likert) (CLASS: 25)

(f) Reasoning skills used to understand physics can be helpful to me in my future career. (Likert) (CLASS: 30) 
(g) The subject of physics has little relation to what I will experience in my future career. (Likert) (CLASS: 35)

3. Medically related questions (Same as in the pre-spring survey)

(a) It is useful for biomedical professionals to understand the basic physics principles of the instrumentation they use in their work? (Likert)

(b) What do you view as the relationship between physics and medicine? (OE)

4. Question asked in fall and winter term

(a) I enjoy discussing topics covered in this course outside of class. (Likert)

(b) The concepts I learned in this course will be valuable in my future career. (Likert)

(c) The problem solving strategies I learned in this course will be valuable in my future career. (Likert)

(d) Is this course required for your major or future graduate program? (Y/N)

(e) Do you think this course should be required for your major or future graduate program? (Likert)

(f) Please explain your answer to the previous question. (OE)

5. Questions only given to students in the reformed course

(a) Including biomedical applications helped me enjoy physics more than if we had used non-biomedical applications (modified from [11]) (Likert)

(b) Including biomedical applications helped me understand physics more than if we had used non-biomedical applications (modified from [11]) (Likert)

(c) It is easy for me to relate to the concepts in the course when they are described by biomedical experts. (Likert)

(d) It is easy for me to relate to the concepts in the course when they are described by physicists. (Likert)

(e) Overall, do you think this course is an improvement in the instruction of introductory physics? (Likert)

(f) Please explain your answer to the previous question. (OE) 


\section{Appendix C.0. Illustration Quizzes Example}

Students had previous to spring term not seen similar questions in their fall and winter term physics courses. It was therefore necessary to introduce the structure of the questions. Before being asked to complete the first illustration question, the instructor provided an example question (Fig. C.1) and reviewed what kind of answer would receive full credit on a sample problem (Fig. C.2). The physical topic chosen for the example was taken from electromagnetism, which was covered in winter term.

The image below depicts a charged particle interacting with a uniform magnetic field. Using the illustration below:

1. Complete the diagram by drawing the path of the charged particle as it enters the uniform magnetic field. Include as much detail as you can, including descriptions of the physical principles that predict the path of the charged particle. (1 pt. for attempting a solution and a bonus 1 pt. for a correct solution)

2. Describe at least one real world application of the physical principle that is used outside of a physics classroom. Include as much detail as you can. (1 pt. for attempting a solution and a bonus 1 pt. for a correct description)

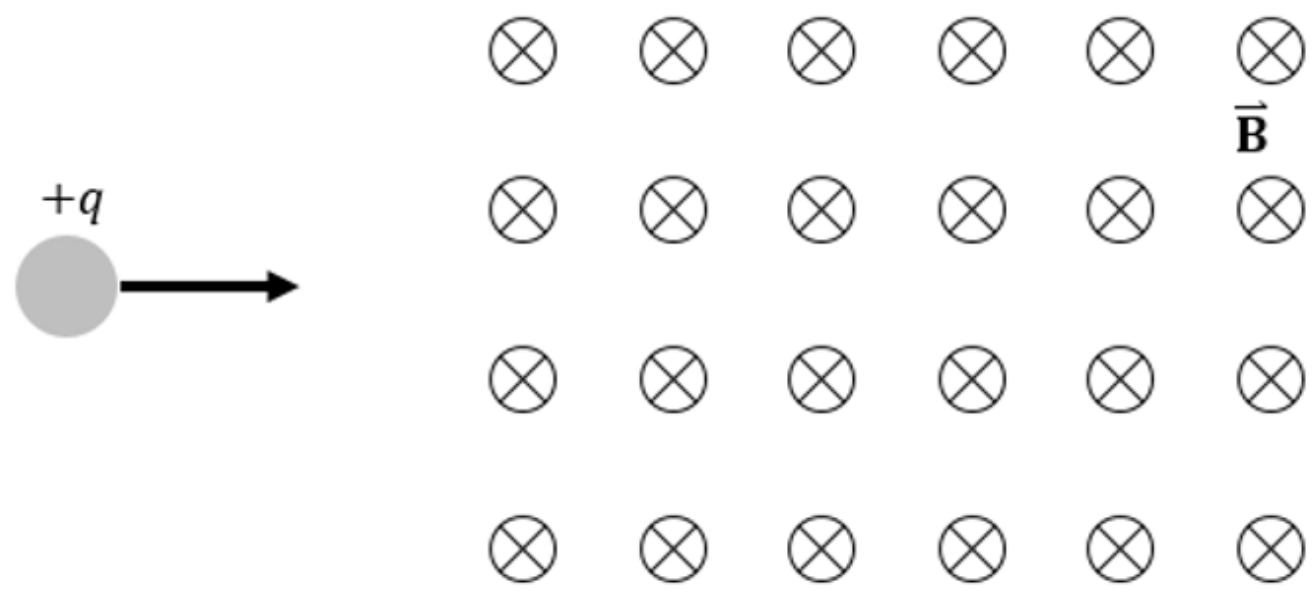

Figure C.1: Illustration given to students as an example of the illustration quizzes. 


\begin{tabular}{|l|l|}
\hline & Full Credit Answer \\
\hline $\begin{array}{l}\text { The path of the charged particle (+q) as it moves } \\
\text { through the magnetic field is described by the } \\
\text { equation } \overrightarrow{\mathbf{F}}=q \overrightarrow{\mathbf{v}} \times \overrightarrow{\mathbf{B}} \text {, which follows the right-hand-rule. } \\
\text { Mass spectrometers use this phenomenon to separate } \\
\text { isotopes. The radius of the circular path depends on } \\
\text { the mass of the particle. So, heavier isotopes will follow } \\
\text { a different circular path than lighter isotopes. }\end{array}$ \\
$\begin{array}{l}\text { +2 points for correctly drawing the path of the charged } \\
\text { particle and describing the magnetic force } \\
+2 \text { points for naming and correctly describing a real- } \\
\text { world application }\end{array}$ & $\begin{array}{l}\text { +1 point for attempting a solution (even though it is } \\
\text { incorrect) } \\
+1 \text { point for correctly naming a real-world application } \\
\text { (even without a description) }\end{array}$ \\
\hline
\end{tabular}

Figure C.2: Samples of answers and grading to the example illustration in Fig. C.1. 


\section{Appendix D.0. Illustration Quiz Revisions}

This section contains the revised form of the illustration quizzes. The revisions were

based on the analysis of the responses to the original questions discussed in Sec. 7.

\section{Sound Waves}

The image depicts a sound wave interacting with a particle in motion. Using the illustration below:

1. Complete the diagram by drawing the path and wave fronts of the wave as it interacts with the particle. Include as much detail as you can. Include descriptions of the physical principles that predict the path of the wave.

( $0.5 \mathrm{pt}$. for attempting a solution and description $+0.5 \mathrm{pt}$. for a correct solution and description)

2. BONUS: Describe one real-world application of this physical principle that is used outside of the classroom. Include as much detail as you can. Include describing how the principle is used. ( $0.25 \mathrm{pt}$. for a correct description of the real-world application)

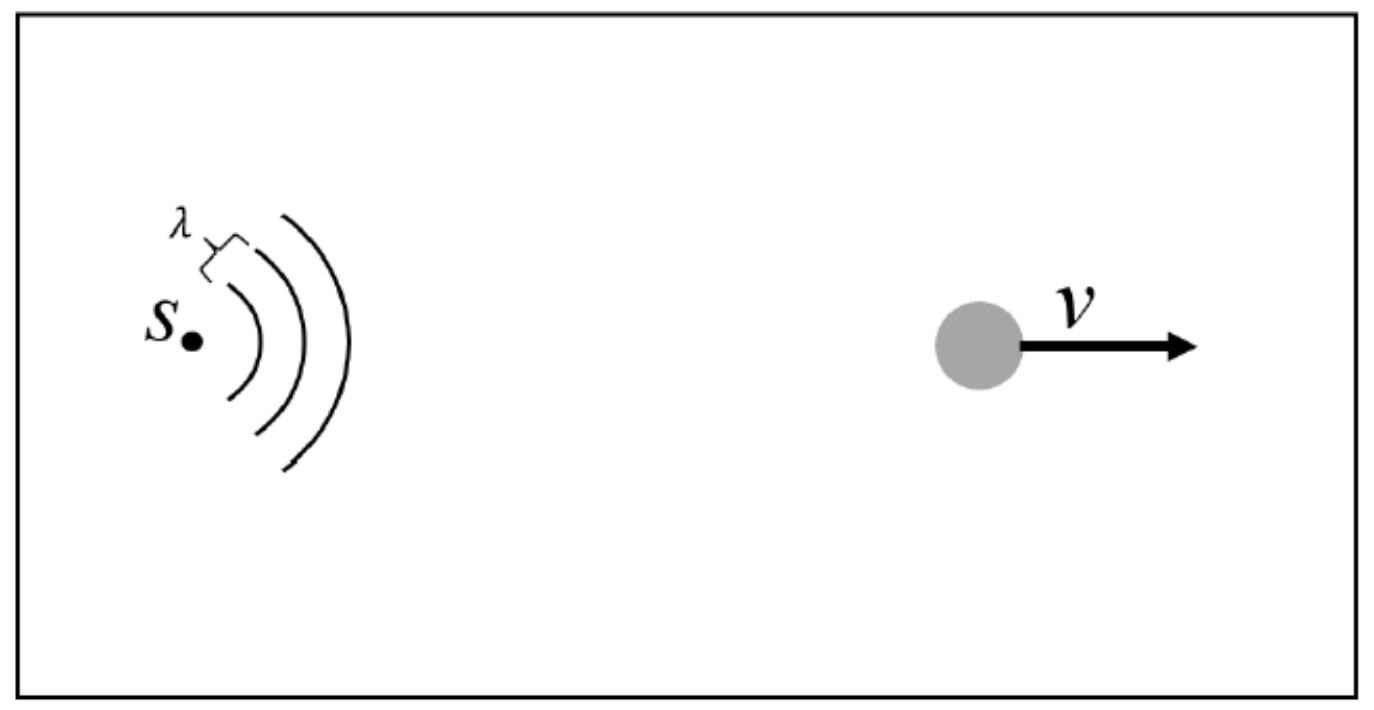

Figure D.1: Proposed revision of the illustration quiz exploring sound waves and Doppler shifts. 


\section{Total Internal Reflection}

The image depicts a light ray interacting with different media $\left(\boldsymbol{n}_{1}>\boldsymbol{n}_{2}\right)$. Using the illustration below:

1. Complete the diagram by drawing the path of the ray as it interacts with the media. Include as much detail as you can. Include descriptions of the physical principles that predict the path of the ray. Include all angle markers.

( $0.5 \mathrm{pt}$. for attempting a solution and description $+0.5 \mathrm{pt}$. for a correct solution and description)

2. BONUS: Describe one real-world application of this physical principle that is used outside of the classroom. Include as much detail as you can. Include describing how the principle is used.

( $0.25 \mathrm{pt}$. for a correct description of the real-world application)

\section{$n_{2}$}

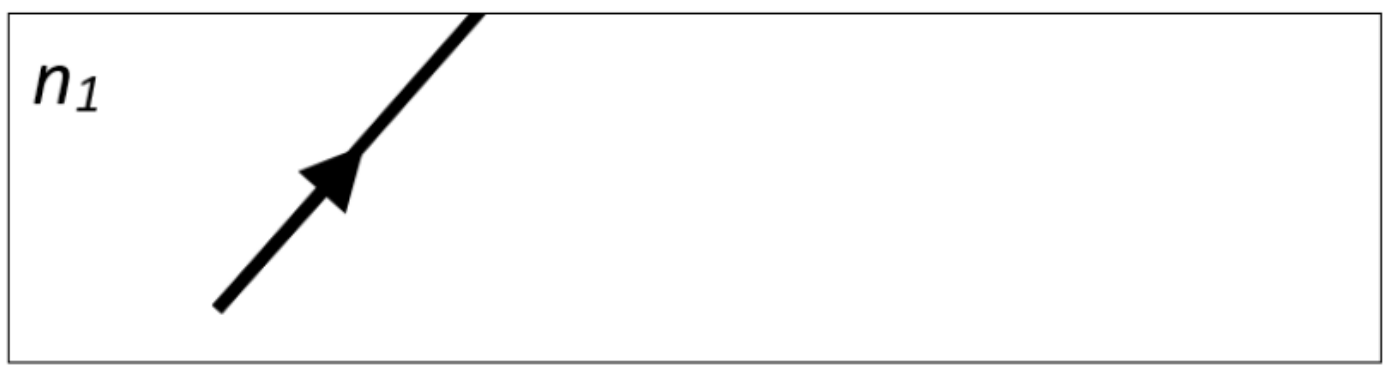

$n_{2}$

Figure D.2: Proposed revision of the illustration quiz exploring total internal reflection. 


\section{Thin Film Interference}

The image depicts a light ray with a wavelength of $550 \mathrm{~nm}$ interacting with different media. Using the illustration below:

1. Complete the diagram by drawing the path of the ray as it interacts with the media. Include as much detail as you can. Include descriptions of the physical principles that predict the path of the ray. Define the thickness of the $n_{2}$ film and the relationship between the three refractive indexes. Include all angle markers.

( $0.5 \mathrm{pt}$. for attempting a solution and description $+0.5 \mathrm{pt}$. for a correct solution and description)

2. BONUS: Describe one real-world application of this physical principle that is used outside of the classroom. Include as much detail as you can. Include describing how the principle is used. ( $0.25 \mathrm{pt}$. for a correct description of the real-world application)

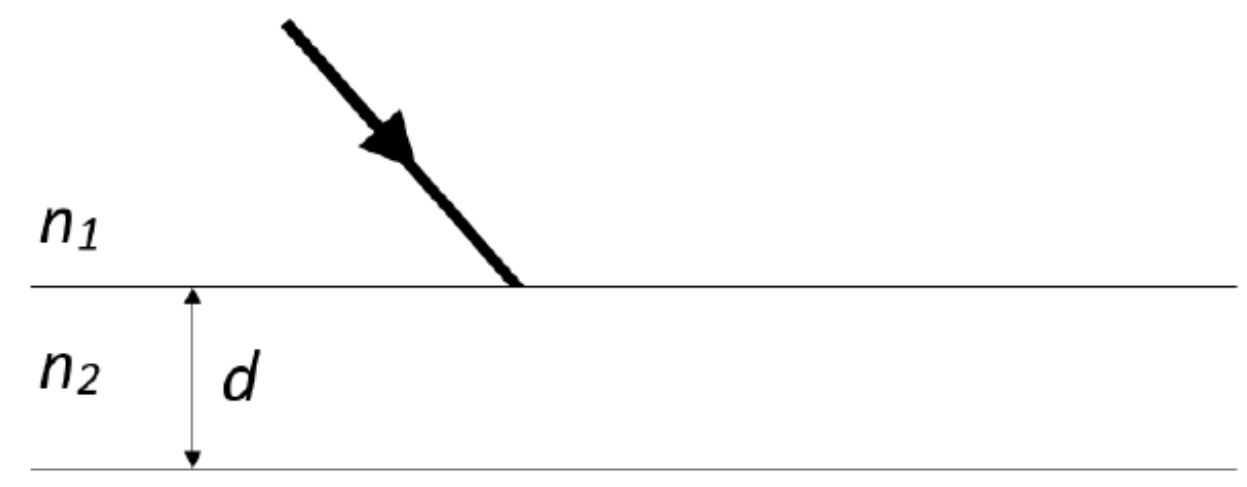

$n_{3}$

Figure D.3: Proposed revision of the illustration quiz exploring thin film interference. 


\section{Image Formation by a Lens}

The image depicts light rays interacting with different media. Using the illustration below:

1. Complete the diagram by drawing the paths of the rays as they interact with the media. Include as much detail as you can. Include descriptions of the physical principles that predict the path of the rays. Define the relationship between the three refractive indexes and Include all angle markers. ( $0.5 \mathrm{pt}$. for attempting a solution and description $+0.5 \mathrm{pt}$. for a correct solution and description)

2. BONUS: Describe one real-world application of this physical principle that is used outside of the classroom. Include as much detail as you can. Include describing how the principle is used.

( $0.25 \mathrm{pt}$. for a correct description of the real-world application)

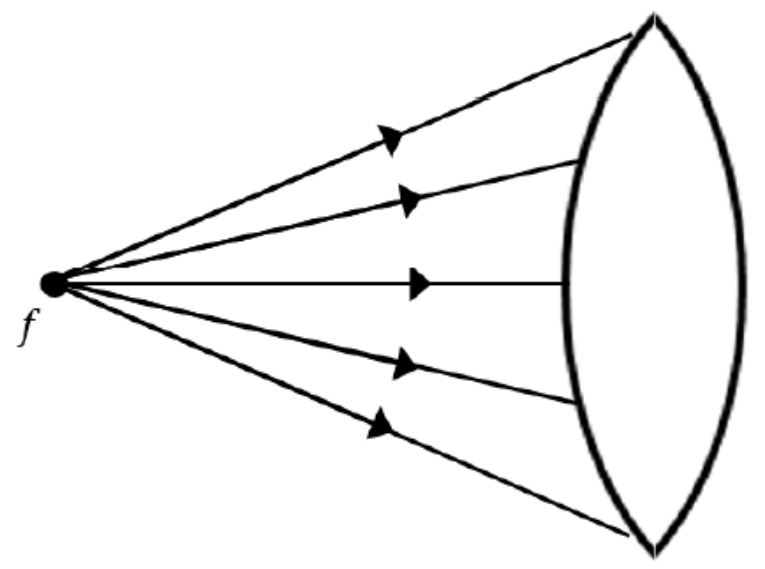

Figure D.4: Proposed revision of the illustration quiz exploring image formation. 


\section{Appendix E.0. Interview Protocol}

The interview protocol was split into four main areas: background, conceptual, attitudinal, and course related questions. All of the questions in each section are included below. Under some of the questions are possible follow up questions or prompts.

\section{Background}

- To start, could you tell me about what subjects and courses you are taking at PSU currently?

- Do these courses promote interdisciplinary learning?

- What are your career goals after college?

- What kinds of knowledge, skill, or experience do you think are important to have for this profession?

\section{Conceptual}

Students will be given a simple diagram and asked to describe the physical processes in detail. The interviewer will look for students' ability to correctly describe the concepts and whether they are making links to biomedicine. (See Sec. 7 for more information on the conceptual illustration quizzes.)

- Tell me about what's happening in this picture.

1. Doppler Example: Possible Prompts

- Do the sound waves change as they move out?

- What happens when the sounds wave and the particle interact?

- How does the original sound compare to the one 'observed' by the particle? 
- Does it make a difference which way the particle is going?

2. Endoscopy Example: Possible Prompts

- How is light traveling through this system?

- What factors influence the path of the beam(s)? (e.g. material composition, incident and critical angle, reflection/refraction)

- Imagine that the $\mathrm{n} 1$ and $\mathrm{n} 2$ materials extend past the edge of this image for a long distance. Tell me about what happens to the beam then? (trying to get the student to describe how fiber optics can guide light beams)

- What are some ways this sort of system is used outside of a physics classroom?

- Is this principle used anywhere in medicine? Could you describe how this principle is used in [student's example]? (only if the students do not mention this themselves)

- How does [student example] compare to this diagram?

\section{Attitudinal}

- Did you take either the fall or winter term algebra based physics courses this year?

- What did you think of those classes? (interesting, useful, difficult, relevant)

- Do you think these classes were important for your planned profession? Why or why not?

- Was there anything in particular that you learned that you think will be useful? Maybe content knowledge or a particular skill?

- Do you ever think about the physics behind things you experience in everyday life? Are there any particular instances where it seems particularly relevant? (Looking to see whether/how students contextualize what they learn. Do they see a link to medicine?)

- Tell me about how you view the relationship between physics and medicine.

- What was your attitude before taking a physics course?

- Did any aspect of the course(s) change your attitude about the relationship between physics and medicine?

- Is there any relation between what youve learned and your future career? 


\section{Course Related}

- Can you tell me about why you took the physics course with the biomedical focus?

- What were your expectations about the course? Do you think your expectations have been right so far? (e.g. flipped classroom, weekly quizzes versus midterms)

- How does this course compare to the fall and winter courses so far?

- What do you think about the biomedical content?

* Has it made the course more interesting?

* Is the biomedical content relevant to your career plans?

* Is the biomedical content authentic or does it feel like it's all physics with a biomedical coating?

* Does the student feel that they are missing out on other physics topics by focusing on biomedicine?

- Were there specific topics from the course you found interesting? What about them was interesting? (Interviewer could probe for conceptual understanding on these topics as well as whether the students see these topics as relevant to their future career.)

- Did the videos with biomedical experts have any impact on your motivation to learn the material?

- Would you have felt as motivated if the same content was taught by a physicist?

- Did you talk about things that were covered in this class with people who werent enrolled in the course? Can you give me some examples?

- How useful do you think this course will be to you in your future career?

- Is there anything about the course you think could be improved?

\section{Conclusion}

- Thank you for participating in this interview. We will be using this information to help us improve the course, so your input has been very helpful.

- Do you have anything you would like to add or any questions for me? 


\section{Appendix F.0. Interview Consent Form}

General Physics: Student Attitudes and Conceptual Understanding Thank you for participating in this interview. The purpose of this interview is to discuss your conceptual understanding of the material in the physics courses you have taken as well as your attitudes toward the courses. The interview will last approximately 40 minutes. Your participation today is completely voluntary. If you feel uncomfortable at any time, you may stop the interview and leave with no penalty. However, your assistance will be greatly appreciated in making this a meaningful research project.

Your answers today will have no impact on your grade. The interview will be audio and video recorded for analysis. Access to the videos will be restricted to the primary researchers in this study only (Elliot Mylott, Warren Christensen, and Ralf Widenhorn). You instructor will not have access to this video. Your instructor will have access to the transcript but only after it has been made completely anonymous and only after final grades have been posted.

If you have any questions regarding your rights as a research subject or any concerns regarding this project, including any dissatisfactions with any aspect of this study, you may report them (confidentially if you so desire) to Human Subjects Research Review Committee, Office of Research and Sponsored Projects, 600 Unitius Bldg., Portland State University, (503) 725-4288 / 1-877-480-4400. If you have any questions about this research project contact Elliot Mylott (emylott@pdx.edu).

Please sign and date to confirm your willingness to participate.

Name (print):

Name (signature):

Date: 


\section{Appendix G.0. Institutional Review Board Approval}

\section{Portland State University HSRRCMemorandum}

To: Ralf Widenhorn

From: HSRRC 2012

Date: June 18, 2012

Re: HSRRC exempt review of your application titled, "Biomedical Physics: Investigation of Effective Teaching Methods" (HSRRC Proposal 122201\#)

Your proposal is exempt from further Human Subjects Research Review Committee review, and you may proceed with the study.

Even with the exemption above, it was necessary by University policy for you to notify this Committee of the proposed research, and we appreciate your timely attention to this matter. If you make changes in the research protocol, the Committee must be notified in writing, and changes must be approved before being implemented.

If you have questions or concerns, please contact the HSRRC at 503-725-2243 or visit us at Research and Strategic Partnerships, Market Center Building, $6^{\text {th }}$ Floor.

co:

Exempt memo 


\section{dי Portland State}

Post Office Box 751

Portland, Oregon 97207-0751

Human Subjects Research Review
503-725-2227 tel

503-725-8170 fax

Committee

hsrrc@lists.pdx.edu

Date: July 15,2014

To: Ralf Widenhorn

From: Karen Cellarius, HSRRC Chair

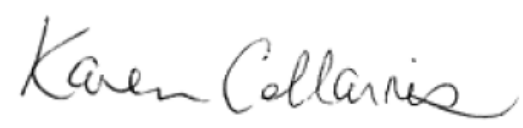

Re: HSRRC approval for your project titled, "Biomedical Physics: Investigation of Effective Teaching Methods" HSRRC Proposal \# 122201

Review Type: Exempt, Category 1

The amendment submitted on $07 / 15 / 2014$ to the above protocol has been reviewed by the Portland State University Institutional Review Board and the Office of Research Integrity. This is a minimal risk research protocol that continues to qualify for an exemption from IRB review under 45 CFR 46. 101(b)(1) for research conducted in established or commonly accepted educational settings, involving normal educational practices, such as research on regular or special education instructional strategies or research on the effectiveness of or the comparison among instructional techniques, curricula, or classroom management methods.

Please note that you will not be required to submit continuing reviews for this protocol, however, you must submit any changes to the protocol to the Office of Research Integrity for assessment to verify that the protocol continues to qualify for exemption.

Your responsibility as a Principal Investigator also includes:

- Obtaining written documentation of the appropriate permissions from public school districts, institutions, agencies, or other organizations, etc., prior to conducting your research

- Notifying the Office of Research Integrity of any change in Principal Investigator

- Notifying the Office of Research Integrity of any changes to or supplemental funding

- Retaining copies of this determination, any signed consent forms, and related research materials for three years after conclusion of your study or the closure of your sponsored research, whichever comes last.

As with all Human Subject Research, exempt research is subject to periodic Post Approval Monitoring review.

If you have any questions regarding your protocol or the review process, please contact the Office of Research Integrity at HSRRC@pdx.edu or (503)725-2227.

Approved: $\quad$ NSF Protocol version 07/15/2014 


\title{
胥 Portland State
}

\author{
Post Office Box 751 -725-2227 tel \\ Portland, Oregon 97207-0751 503-725-8170 fax \\ Human Subjects Research Review Committee \\ hsrrc@lists.pdx.edu
}

Date: $\quad$ May 28, 2015

To: $\quad$ Ralf Widenhorn / Grace Van Ness

From: Karen Cellarius, HSRRC Chair

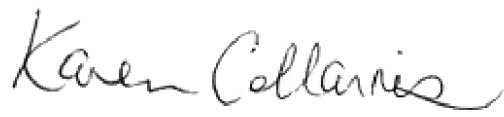

Re: HSRRC approval for your project titled, "Biomedical Physics: Investigation of Effective Teaching Methods" HSRRC Proposal \# 122201

Review Type: Exempt, Category 1

The amendment submitted on $05 / 13 / 2015$ to the above protocol has been reviewed by the Portland State University Institutional Review Board and the Office of Research Integrity. This is a minimal risk research protocol that continues to qualify for an exemption from IRB review under 45 CFR 46. 101(b)(1) for research conducted in established or commonly accepted educational settings, involving normal educational practices, such as research on regular or special education instructional strategies or research on the effectiveness of or the comparison among instructional techniques, curricula, or classroom management methods.

Please note that you will not be required to submit continuing reviews for this protocol, however, you must submit any changes to the protocol to the Office of Research Integrity for assessment to verify that the protocol continues to qualify for exemption.

Your responsibility as a Principal Investigator also includes:

- Obtaining written documentation of the appropriate permissions from public school districts, institutions, agencies, or other organizations, etc., prior to conducting your research

- Notifying the Office of Research Integrity of any change in Principal Investigator

- Notifying the Office of Research Integrity of any changes to or supplemental funding

- Retaining copies of this determination, any signed consent forms, and related research materials for three years after conclusion of your study or the closure of your sponsored research, whichever comes last.

As with all Human Subject Research, exempt research is subject to periodic Post Approval Monitoring review.

If you have any questions regarding your protocol or the review process, please contact the Office of Research

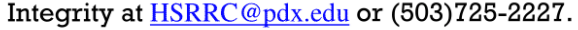

Approved: $\quad$ Addition of Elliot Mylott to the study personnel. 